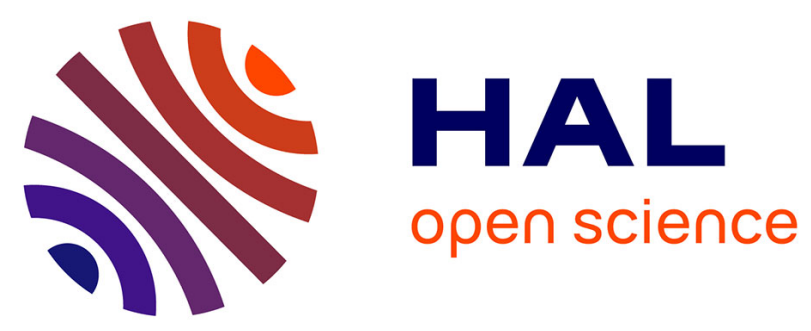

\title{
TrackDip: a multi-scale processing of dipmeter data. Method, tests, and field example for 3D description of gravity-driven deformations in the Eocene foreland basin of Ainsa, Spain
}

Christophe Basile, Arnaud Pécher, Mathieu Corazzi, Francis Odonne, Agnès Maillard, Elie Jean Debroas, Pierre Callot

\section{To cite this version:}

Christophe Basile, Arnaud Pécher, Mathieu Corazzi, Francis Odonne, Agnès Maillard, et al.. TrackDip: a multi-scale processing of dipmeter data. Method, tests, and field example for 3D description of gravity-driven deformations in the Eocene foreland basin of Ainsa, Spain. 2008. hal-00124633v2

\author{
HAL Id: hal-00124633 \\ https://hal.science/hal-00124633v2
}

Preprint submitted on 12 Mar 2008

HAL is a multi-disciplinary open access archive for the deposit and dissemination of scientific research documents, whether they are published or not. The documents may come from teaching and research institutions in France or abroad, or from public or private research centers.
L'archive ouverte pluridisciplinaire HAL, est destinée au dépôt et à la diffusion de documents scientifiques de niveau recherche, publiés ou non, émanant des établissements d'enseignement et de recherche français ou étrangers, des laboratoires publics ou privés. 


\title{
TrackDip: a multi-scale processing of dipmeter data. Method, tests, and field example for 3D description of gravity-driven deformations in the Eocene foreland basin of Ainsa, Spain
}

\author{
C. Basile ${ }^{1 *}$, A. Pecher ${ }^{1}$, M. Corazzi ${ }^{1}$, F. Odonne ${ }^{2}$, A. Maillard ${ }^{2}$, E.J. Debroas ${ }^{2}$, P. Callot ${ }^{2}$
}

\author{
1 : Laboratoire de Géodynamique des Chaînes Alpines, CNRS-UMR 5025, Observatoire des Sciences de l'Univers de \\ Grenoble, Université Joseph Fourier, BP 48, 38041 Grenoble Cedex, France. Fax 33476514058 \\ 2 : Laboratoire de Mécanismes de Transfert en Géologie, CNRS-UMR 5563, Observatoire Midi Pyrénées, Université Paul \\ Sabatier, 14 Avenue Edouard Belin, 31400 Toulouse, France. Fax 33561332560. \\ * Corresponding author : cbasile@ujf-grenoble.fr
}

\begin{abstract}
This paper presents TrackDip, a new method for dipmeter data processing. This method selects, localizes and measures significant tilts from the comparison at various scales of the changes of orientation of sedimentary beddings. We tested this method on simple cases (unconformity, gradual tilt, successive tilts, without and with additional random noise) and on a field example, along three sections in the Eocene Sobrarbe delta, Ainsa foreland basin, northern Spain. For synthetic data sets, the method clearly identifies and measures the introduced tilts.

On the field, sedimentary and tectonic structures, especially three main syn-sedimentary sliding surfaces (S1 to S3), were successfully identified from processed dipmeter data. The tilt axis are mainly trending N-S in sandstones, associated to westward transport of sediments. The sliding surfaces S1 and S3 correspond to E-W-trending tilt axis, tentatively correlated either to the flexural subsidence of the basin, or to anticline growth during sedimentation south of the studied area. Combination of these sedimentary and tectonic directions results in a NE-SW striking submarine slope, which locally controls the gravitydriven deformations, especially on the S2 sliding surface. Finally, NNW-SSE-trending tilts above the uppermost S3 sliding surface are interpreted as the result of infilling on the side of the scar produced by sliding.
\end{abstract}

Keywords : dipmeter, tilt, processing, gravity-driven deformations, foreland basin

\section{Introduction}

Among downhole logging tools, dipmeters represent a class of tools designed to measure the orientation, i.e. both dip angles and azimuth (dip direction) of bedding planes intersected by a borehole. While boreholes are onedimensional, dipmeter data allow to describe 3-D geometry of sedimentary units, and bring crucial information on all processes involving changes of orientation in sediment bedding, such as deposition in flows (e.g. Luthi and Banavar, 1988 ; Höcker et al., 1990 ; Donselaar and Schmidt, 2005), deformations during and after sedimentation (e.g. Hesthammer and Fossen, 1998), differential subsidence. It is noteworthy that these changes of orientation occur at various space and time scales, and that their effects are merged in a single record.

The word dipmeter refers only to dip measurements, and little has been done to fully use these three-dimensional measurements. The classical tadpole graphical display of dip and azimuth as a function of depth (Serra, 1989) underlines the dip variations, but azimuth variations are difficult to read. Interpretations often used mainly dip variations (Gilreath, 1987; Serra, 1989). Similarly, processing methods either used only dip measurements (Hurley, 1994), or are restricted to a given scale (point to point analysis : Berg, 1998 ; folded structures : Bengtson, 1981).
In this article, we present a new method, named TrackDip, to process bedding attitudes from outcrops in order to identify at various scales the successive changes of orientations in a sedimentary section. While designed to process dipmeter logging data, we tested this method on a field example, in the Eocene foreland basin of Ainsa (Spain). This test allows a direct comparison between interpretations from dipmeter analysis and outcrop exposures.

\section{TrackDip processing of dipmeter data}

The processing methodology described herein is a formalized version of the empirical process used by Basile (2000) on logging data. Annexes 1 to 4 present the successive stages of processing for the three datasets analysed in this paper.

The dipmeter data set consists in a series of measurements of elevation, azimuth and dip angle of stratigraphic bedding surfaces (e.g. Annex 1). Processing is based on a multi-spectral analysis, where the changes of bedding attitude are compared and tracked between various scales (Figure 1). It is important to note that this processing only applies to stratigraphic surfaces. It has no meaning if sedimentary beddings and tectonic structures are mixed in the dipmeter data set. 

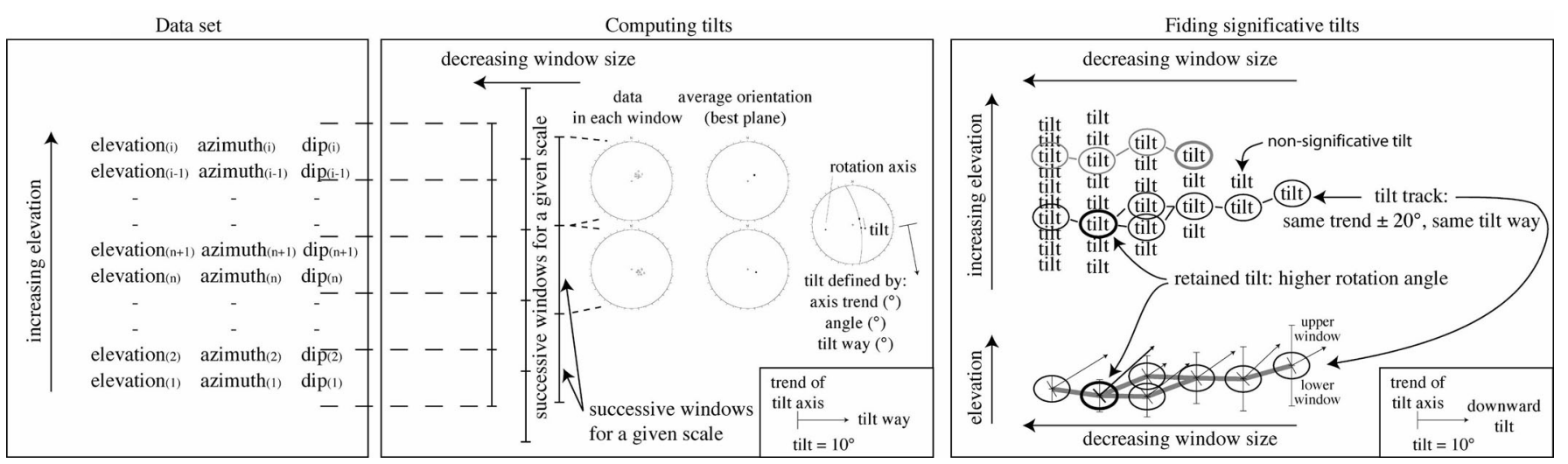

Figure 1 : Successive steps of dipmetry processing : data set, computing tilts as a function of scale, identifying significant tilts. Stereographic projections : Wulff nets, lower hemisphere. See text for details. Tilts and tracked tilts are shown as in Figures 3 to 9 and 15 to 17 ; the tilt way arrow indicates the downward tilt.

\subsection{Investigated windows}

A given scale (e.g. $1 \mathrm{~m})$ defines a window size $(1 \mathrm{~m}$ in this example). For this given scale the data set is cut in successive windows, positioned symmetrically by reference to the centre of the depth interval (e.g. eleven meter-scale windows from 10 to $21 \mathrm{~m}$ for a $10.25-20.75 \mathrm{~m}$ interval). The positions of windows depend only on the scale and on the position of the first and last data. The upper and lower limit of each window define its position.

\subsection{Best planes}

In each window, we compute a best plane, i.e. an average of the bedding plane orientations contained in this window. Most of the time, this best plane has no geological meaning, as it may average data from distinct populations like above and below an unconformity. For the smaller scales, there is only one data in most windows, and we use this data as the best plane.

\subsection{Tilts}

A change of attitude (tilt) is then calculated between two successive and adjacent windows (Figure 1, Annex 2). As for the best planes, computed tilts may not have a geological meaning, as they may mix together several independent geological tilts. Each tilt is defined by the trend of the rotation axis, a rotation angle, and a tilt way (Annex 2) ; it is located by the position of the limit between the two consecutive windows. Defining the rotation axis only by their trends restricts this processing to moderately dipping (less than $45^{\circ}$ ) sections. The tilt way is defined by the azimuth of the lower plane when both upper and lower planes are rotated to bring the upper one horizontal (Figure 2A). It is important to notice that the tilt way does not indicate absolute but relative tilt, by reference to the upper layer. For example, depending on the dip of the upper layer at the time of sedimentation, a similar tilt way towards East can be interpreted either as a dune dipping East below an horizontal surface, or as an horizontal surface below a dipping West prograding clinoform (Figure 2B).

\subsection{Tracking rotations}

Investigation scales vary from the smallest depth-interval between two data (often at centimetre scale), up to the size of the depth interval for the whole data set (few tens to hundred meters). In order to make the comparison between the various scales easier, we used an arbitrary fixed ratio of $10^{0.1}$ between consecutive scales, investigating ten scales between 10 centimetres and 1 meter, between 1 meter and 10 meter, etc. Computing the tilts between all the adjacent windows for all investigation scales results in matrixes of rotation axis trend, rotation angle, and tilt way as a function of depth and window size (Figure 1, Annex 2). Tracking rotations is a process that identifies the most significant tilts in these matrixes.

Whatever the scale and the geological process are, every tilt can be considered either as a sudden tilt (change of orientation between two data) or as a gradual tilt around a single rotation axis between more than two data. In both cases, we call this tilt 'event', and 'noise' the other changes of orientation, that can be instrumental errors or the adjacent geological event. This terminology applies to each tilt, which is an event by itself, and a noise for other tilts. A random noise has a random distribution around an average value, and the best plane does not vary significantly from this average value when the window size increases.

\subsubsection{Sudden tilt}

\subsubsection{Best measurement}

For a sudden tilt, the event is correctly measured when it fits the boundary between two windows. For this position and for random noise, the best measurement of the tilt can be obtained for the widest windows, which represent the best average. If the noise is not random, and results from neighbouring events, then the best measurement of the tilt is obtained for the widest windows that do not cover the adjacent events.

\subsubsection{Best location}

In fact the main problem is that the position of the event is not known in advance. If a window straddles this event, it contains data from the two groups separated by the tilt. Its best plane differs from these two groups, but the tilt between this best plane and each of the two groups has an axis similar to the searched tilt, but smaller rotation angle. Consequently, the event may be identified by constant parameters (axis trend, way) for various and successive window sizes. As the rotation angle increases when the mixing between the two groups decreases, the best location is obtained for the highest rotation angle. 


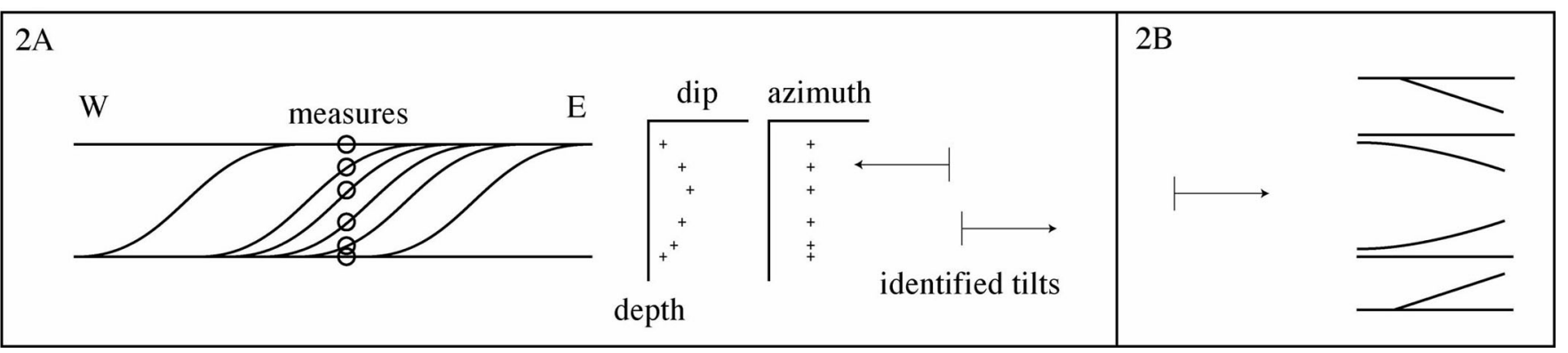

Figure 2. A : a single sedimentary structure may be identified by two tilts with opposite tilt ways. Tilt symbol as in Figure 3 to 9 and 15 to 17 . B : various interpretations of the same tilt : the tilt way does not give indications on sedimentary surfaces intersection ; the same tilt way can correspond either to downlap or toplap, but also to curved surfaces.

\subsubsection{Gradual tilt}

Gradual tilt can be considered as a succession of adjacent sudden tilts with similar rotation axis and ways. In this case, the best location match the whole set of adjacent windows, at the scale where the sum of rotation angles is maximum. Then the best measurement consists in the average rotation axis and ways, and rotation angle is the sum of rotation angles.

\subsubsection{Process}

The significant events are tracked from the largest to the smallest window sizes, searching rotations characterized by significant rotation angles $\left(>3^{\circ}\right)$, similar axis trend and similar tilt way in the same depth intervals. We consider trends to be similar at $\pm 20^{\circ}$, but this value, as the rotation angle cut-off, can be modified (see below $\S 3$ ). We retain only as significant events the rotations that can be tracked in at least three successive window sizes without similar data sets (Annex 3). For a given scale, when two successive tilts (i.e. three successive windows) have similar axis trend and tilt way, we define the tilt by the average trend and by the sum of tilt angles. Among all the rotations tracking a single event for various window sizes, we retain arbitrary the higher rotation angle (Annex 4).

\section{Methodological tests (Table 1)}

In this section, we present few tests of TrackDip performed on synthetic data sets. Each data set contains 200 measurements in a 100 m-thick section. The depth of each point has been randomly distributed within this section.

\subsection{Test1 (Figure 3)}

In this test, a single unconformity occurs between 120.28 and $121.16 \mathrm{~m}$. The unconformity represents a $7.78^{\circ}$ tilt around a rotation axis trending $\mathrm{N} 53.6^{\circ}$. In this example, TrackDip perfectly localizes and measures the event (Table 1). As expected, the maximum rotation angle is found where the boundary between two windows fits the depth of the unconformity. As there are no other changes in orientation, the unconformity is the only event identified by TrackDip.

\subsection{Test 2 (Figure 4)}

Test 2 is similar to Test1, but with a random change of orientation on each data. Dips are randomly modified $\pm 4^{\circ}$, and azimuths are randomly modified $\pm 4 \% \sin ($ dip). These changes approximate a random distribution around an average orientation. The resulting tilts between adjacent data that belongs to the same group (above or below the unconformity) are smaller than $12^{\circ}$. As for Test 1 , TrackDip localizes and measures the unconformity, with a small difference for the rotation angle, and a very small difference for the rotation axis trend (Table 1). Other events are also tracked for smaller window sizes, and for smaller scale ranges. These events are related to the most important random changes of orientation, like the one at $142 \mathrm{~m}$ (Figure 4). They are tracked for small window sizes, when windows contain only few data (usually less than seven). Around $154.5 \mathrm{~m}$, a double event is tracked, with similar rotation axis and angle, but with opposite ways (Figure 4). This double event can be related to a single data located between them, which is significantly different from the neighbouring data.

The same Test 2 has been processed using various cutoffs (Figure 4). Decreasing the minimum rotation angle from $4^{\circ}$ to $2^{\circ}$ does not change the tracking of the unconformity, but tracks an increasing number of smaller events, almost all for small windows containing less than seven data. On the contrary, reducing the allowed change of rotation axis from $\pm 30^{\circ}$ to $\pm 10^{\circ}$ reduces the number of tracked events, and the continuity of tracking. For example, for $\mathrm{a} \pm 10^{\circ}$ change, the unconformity is not identified by a single track, but by two tracks at the same depth but for different window scales (Table 1).

\subsection{Test 3 and Test 4 (Figures 5 and 6)}

Test 3 and Test 4 are also similar to Test 1 but dips are randomly modified $\pm 8^{\circ}$ and $\pm 12^{\circ}$, respectively. Azimuths are randomly modified $\pm 8 \% \sin (\operatorname{dip})$ and $\pm 12 \% \sin (\operatorname{dip})$, respectively. This increases the changes of orientations related to the random distribution to more than $20^{\circ}$ and $30^{\circ}$, respectively. Consequently, an increasing number of tilts are tracked, and they are not restricted to small window sizes (Fig. 6). Increasing the random noise also moves the identification of the unconformity to wider windows, and decreases the precision of both localization and measurement (Table 1).

\subsection{Test5 (Figure 7)}

In this test, we introduced a gradual tilt, then data were randomly modified (Table 1). The gradual tilt is well localized and measured by TrackDip, but for wider windows than the interval where the tilt occurs (Table 1), because the best computation requires the comparison of 
Marine \& Petroleum Geology, accepted

\begin{tabular}{|c|c|c|c|c|c|c|c|c|c|c|c|c|}
\hline & & & Introduced & & & & Cut-offs & & Observed & & & \\
\hline & Figure & $\begin{array}{l}\text { Type } \\
\text { of tilt }\end{array}$ & $\begin{array}{l}\text { Depth } \\
(\mathrm{m})\end{array}$ & $\begin{array}{c}\text { Angle } \\
\left({ }^{\circ}\right)\end{array}$ & $\begin{array}{l}\text { Axis } \\
\left({ }^{\circ}\right)\end{array}$ & $\begin{array}{c}\text { Random } \\
\text { noise }\end{array}$ & $\begin{array}{c}\text { Tilt angle } \\
\left({ }^{\circ}\right)\end{array}$ & $\begin{array}{c}\text { Axis } \\
\text { trend } \\
\left({ }^{\circ}\right)\end{array}$ & $\begin{array}{l}\text { Depth } \\
(\mathrm{m})\end{array}$ & $\begin{array}{l}\text { Window } \\
\text { scale }(\mathrm{m})\end{array}$ & $\begin{array}{c}\text { Angle } \\
\left({ }^{\circ}\right)\end{array}$ & $\begin{array}{l}\text { Axis } \\
\left({ }^{\circ}\right)\end{array}$ \\
\hline Test1 & 3 & Sudden & 120.72 & 7.78 & 53.6 & 0 & 4 & 30 & 120.76 & 2 & 7.78 & 53.6 \\
\hline \multirow[t]{3}{*}{ Test2 } & 4 & Sudden & 120.72 & 7.78 & 53.6 & $\pm 4^{\circ}$ & 3 & 20 & 120.8 & 2.52 & 9.15 & 54.9 \\
\hline & & & & & & & 3 & 10 & $\begin{array}{l}119.95 \\
120.7 \\
\end{array}$ & $\begin{array}{l}19.95 \\
0.79 \\
\end{array}$ & $\begin{array}{c}7.54 \\
8.6 \\
\end{array}$ & $\begin{array}{c}55.5 \\
48 \\
\end{array}$ \\
\hline & & & & & & & & & & & & \\
\hline Test3 & 5 & Sudden & 120.72 & 7.78 & 53.6 & $\pm 8^{\circ}$ & 3 & 20 & 119.76 & 19.95 & 8.64 & 51.5 \\
\hline Test4 & 6 & Sudden & 120.72 & 7.78 & 53.6 & $\pm 12^{\circ}$ & 3 & 20 & $\begin{array}{c}118 \\
(94.22-141.77)\end{array}$ & 15.85 & 8.24 & 85.8 \\
\hline Test5 & 7 & Gradual & $169.7-173.7$ & 15.69 & 94.9 & $\pm 4^{\circ}$ & 3 & 20 & $\begin{array}{c}171.77 \\
(162.31-181.24)\end{array}$ & 6.31 & 15.56 & 87.2 \\
\hline \multirow[t]{5}{*}{ Test6 } & 8 & Sudden & 112.8 & 11 & 75 & 0 & 2 & 30 & 112.8 & 0.398 & 11 & 74.8 \\
\hline & & Gradual & $124.87-131.25$ & 15 & 348 & 0 & & & $\begin{array}{c}127.6 \\
(122.6-132.6) \\
\end{array}$ & 2.512 & 12.88 & 351.93 \\
\hline & & Gradual & $131.25-136.16$ & 15 & 348 & 0 & & & $\begin{array}{c}133.85 \\
(130.1-137.6)\end{array}$ & 2.512 & 11.92 & 352.2 \\
\hline & & Gradual & $144.17-154.5$ & 5 & 240 & 0 & & & 150.2 & 12.59 & 3.8 & 237.8 \\
\hline & & Sudden & 183.38 & 17 & 200 & 0 & & & $\begin{array}{c}166.01 \\
(118.6-203.4) \\
183.64 \\
\end{array}$ & $\begin{array}{l}31.623 \\
0.631 \\
\end{array}$ & $\begin{array}{r}20.36 \\
15.77 \\
\end{array}$ & $\begin{array}{r}207.15 \\
197.9 \\
\end{array}$ \\
\hline & & & & & & & & & & & & \\
\hline \multirow[t]{5}{*}{ Test7 } & 9 & Sudden & 112.8 & 11 & 75 & $\pm 4^{\circ}$ & 2 & 30 & $\begin{array}{c}110.48 \\
(98.57-122.4)\end{array}$ & 7.943 & 12.7 & 78.25 \\
\hline & & Gradual & $124.87-131.25$ & 15 & 348 & $\pm 4^{\circ}$ & & & $\begin{array}{c}127.64 \\
(120.13-135.16) \\
\end{array}$ & 5.012 & 13.03 & 341.1 \\
\hline & & Gradual & $131.25-136.16$ & 15 & 348 & $\pm 4^{\circ}$ & & & $\begin{array}{c}133.83 \\
(131.94-135.72)\end{array}$ & 1.259 & 12.46 & 348.95 \\
\hline & & Gradual & $144.17-154.5$ & 5 & 240 & $\pm 4^{\circ}$ & & & 152.71 & 2.512 & 3.53 & 270.9 \\
\hline & & Sudden & 183.38 & 17 & 200 & $\pm 4^{\circ}$ & & & $\begin{array}{c}166.01 \\
(118.6-203.4) \\
183.4 \\
(178.66-188.15) \\
\end{array}$ & $\begin{array}{l}31.623 \\
3.162\end{array}$ & $\begin{array}{l}19.78 \\
16.7\end{array}$ & $\begin{array}{c}207 \\
188.5\end{array}$ \\
\hline
\end{tabular}

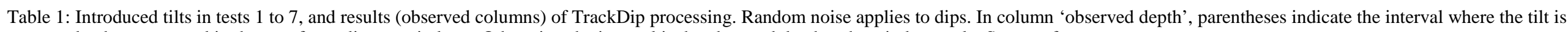
measured, when measured in three or four adjacent windows. Otherwise, the interval is the observed depth \pm the window scale. See text for comments. 


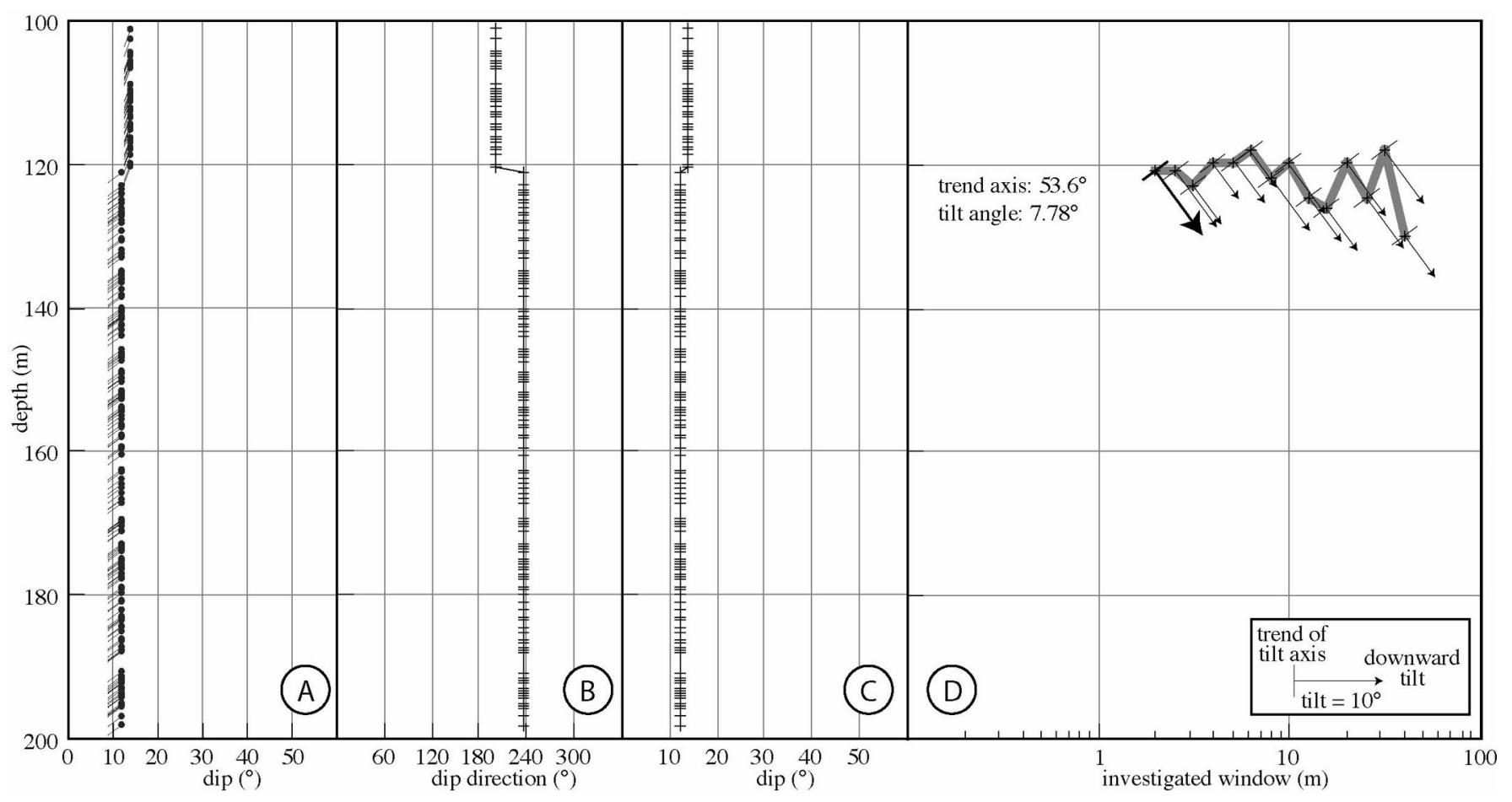

Figure 3: Test1: Display of data and TrackDip results. A : tadpole representation of bedding attitudes. B : azimuth of beds. C : dip of beds. D : TrackDip results : the tracked tilt event (thick grey line) is shown as a function of the investigated window size (logarithmic scale). Tilts are shown for each investigated window ; the bold tilt is retained as the most significant tilt for the tracked event (see text and Figure 1). Cut-offs are $4^{\circ}$ for the rotation angle and $\pm 30^{\circ}$ for the rotation axis trend.

data that are outside the tilting interval. When compared to sudden tilts (Figure 4), the gradual tilt is tracked only for wide windows, because differences of orientation between adjacent data are small, and below the angle cut-off for small-size windows. Some of these small-size windows identify parts of the gradual tilt, with similar rotation axis but smaller rotation angles (Figure 7).

\subsection{Test6 (Figure 8)}

In this test, we introduce several tilts in the same interval. Two of them are sudden tilts, three are gradual tilts (Table 1 and Figure 8). The three deeper tilts have similar rotation axis and the same tilt ways. As for Test1, the sudden tilts are perfectly localized and measured for the smallest window size. However, at large widow sizes, the three deeper tilts merge together in a single and more important tilt, which is not representative of a single event, but of the large-scale effect of three events. As seen for Test5, gradual tilts are best identified at scales larger than the tilt intervals.

\subsection{Test7 (Figure 9)}

Test7 is similar to Test6, with a random change of orientation on each data. Dips are randomly modified $\pm 4^{\circ}$, and azimuths are randomly modified $\pm 4 \% \sin (\mathrm{dip})$. As for Test 2 compared to Test1, there are numerous tracked events related to the random modification. The five introduced tilts are localized and measured like in Test6, but for wider windows, containing more than 7 data (Table $1)$.

\subsection{Synthesis}

From these tests, it appears that TrackDip correctly identifies, localizes and measures single or multiple tilts, either sudden or gradual. Sudden tilts are easier to detect than gradual tilts, that requires lower cut-offs during the processing. The identification is easier for wide windows ; the localization and measurement occur at narrow windows with no or limited random changes, and at wider windows when the dispersion of orientations increases. However, the size of the windows can not be used as an automatic cutoff : the events tracked for small window sizes exist. In the presented tests, they are related to random dispersion of orientation, but in real studies they can be related to single geological events. Anyway the most significant events always appear as those that can be tracked in a maximum of consecutive scales.

The main limit of TrackDip is that it does not process the superposition of tilts. When similar rotations are closed one to another, they are correctly identified at small window scales, but combined at large window scales. Similarly, superposition of tilts in the same interval may be averaged at large windows. For example, meter-scale synsedimentary tilts can be deformed by a later folding at a kilometre-scale. TrackDip can identify both tilts at both scales, but the meter-scale measurements reflect the superposition of the two tilts.

\section{Field test}

In the following section, we present a field application of TrackDip, to perform several geological tests :

- Efficiency: does this method correctly localise and measure the sedimentary or structural structures observed on the field?

- Reproducibility: does this method give similar results when performed in similar sections? 
Marine \& Petroleum Geology, accepted
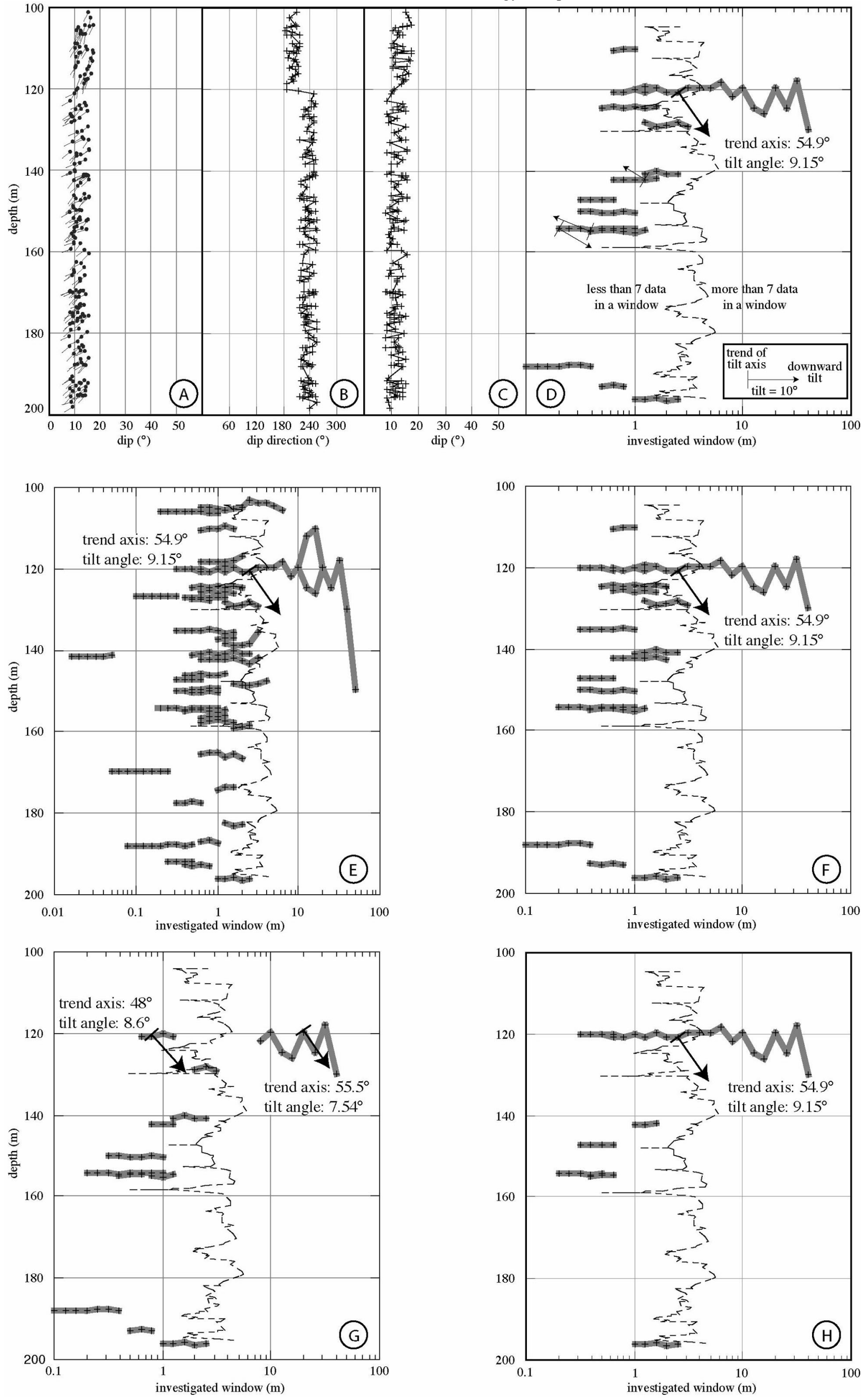
Figure 4: Test2: Display of data and TrackDip results. A to D : same legend as for Figure 3. In D, the only tilt shown in bold is the most significant tilt for the tracked event that corresponds to the introduced tilt. The other shown tilts are discussed in the text. The dotted line indicates the size of the windows containing seven data. Cut-offs are $3^{\circ}$ for the rotation angle and $\pm 20^{\circ}$ for the rotation axis trend. $\mathrm{E}$ to $\mathrm{H}$ : same legend as for $\mathrm{D}$, with different cut-offs $\left(\mathrm{E}: 2^{\circ}\right.$ and $\pm 30^{\circ} ; \mathrm{F}: 3^{\circ}$ and $\pm 30^{\circ} ; \mathrm{G}: 3^{\circ}$ and $\pm 10^{\circ} ; \mathrm{H}: 4^{\circ}$ and $\pm 30^{\circ}$ ). See text for comments.

- Predictive ability: is this method able to identify a structure not observed directly in the logged section, but in its vicinity.

\subsection{Geological background}

The studied sections are located in the Ainsa basin, which represents with Tremp and Jaca basins the Eocene foreland basin south of the Pyrenean belt (Puigdefabregas et al., 1991 ; Figure 10). Lithospheric flexure induced an increasing subsidence towards NorthEast to NorthNorthEast. During basin formation and infilling, the development of a thrust ramp (Sierra de Montsec) induced an East-West trending drainage, and the development of the important Sobrarbe delta feeding the Ainsa marine basin from the South East (Figure 10, Dreyer et al., 1999). The propagation of the lateral thrust ramps induced intrabasinal North-South-trending growth anticlines, from East to West, and from the oldest to the youngest: Mediano, Arcusa, Boltana anticlines (Figure 10). These anticlines locally imposed a north-northwestward progradation of the delta. Finally, the Ainsa basin has been unconformably covered by Middle to Upper Eocene continental sediments of Jaca basin (Figure 10).

The studied sections belong to the proximal part of the Sobrarbe delta, at the top of Las Gorgas composite sequence (Dreyer et al., 1999) : the shallower facies are nummulitic limestones, either in situ or resedimented in submarine dunes or sand channels in the delta shoreface ; deeper facies are siltstones and mudstones from the prodelta. In the studied area, the delta front has been affected by large-scale collapses. Dreyer et al. (1999) interpret these collapses as a result of tilting on the flank of a North-South-trending Arcusa growth fold, although they notice that tilting seems to occur towards NorthNorthWest. It is noteworthy that the Boltana anticline was not well-developed at the time of deposition of the studied sections, but appeared more recently as a growth fold. Callot et al. (Submitted) identified three sliding surfaces (S1 to S3) in the studied area (Figure 11). S1 and S3 truncate the underlying sediments, with marked angular unconformities (Figures 12 and 13). S1 and S2 are associated to numerous normal faults and clastic dykes that indicate northwestward slides (Callot et al., submitted; Figure 14). From the orientation of S3 and its vanishing towards north (Figures 11 and 13), a northward slide is postulated for this surface (Callot et al., submitted).

\subsection{Measurements}

On three outcrops, we systematically measured available and accessible attitudes of beds along three sections on the right bank of Rio Ena, between barrancos Mazana and Solano (Figures 11 to 13). The measurements were reported on a vertical log. Elevations are given by reference to the first measure of the section. The error on the difference of depth between two consecutive measures is estimated to $20 \%$. As we measured all observed bed surfaces, measurements are closer in sandstones where individual strata are centimetre- to decimetre-thick, than in mudstones where clear beddings are sparse, and often

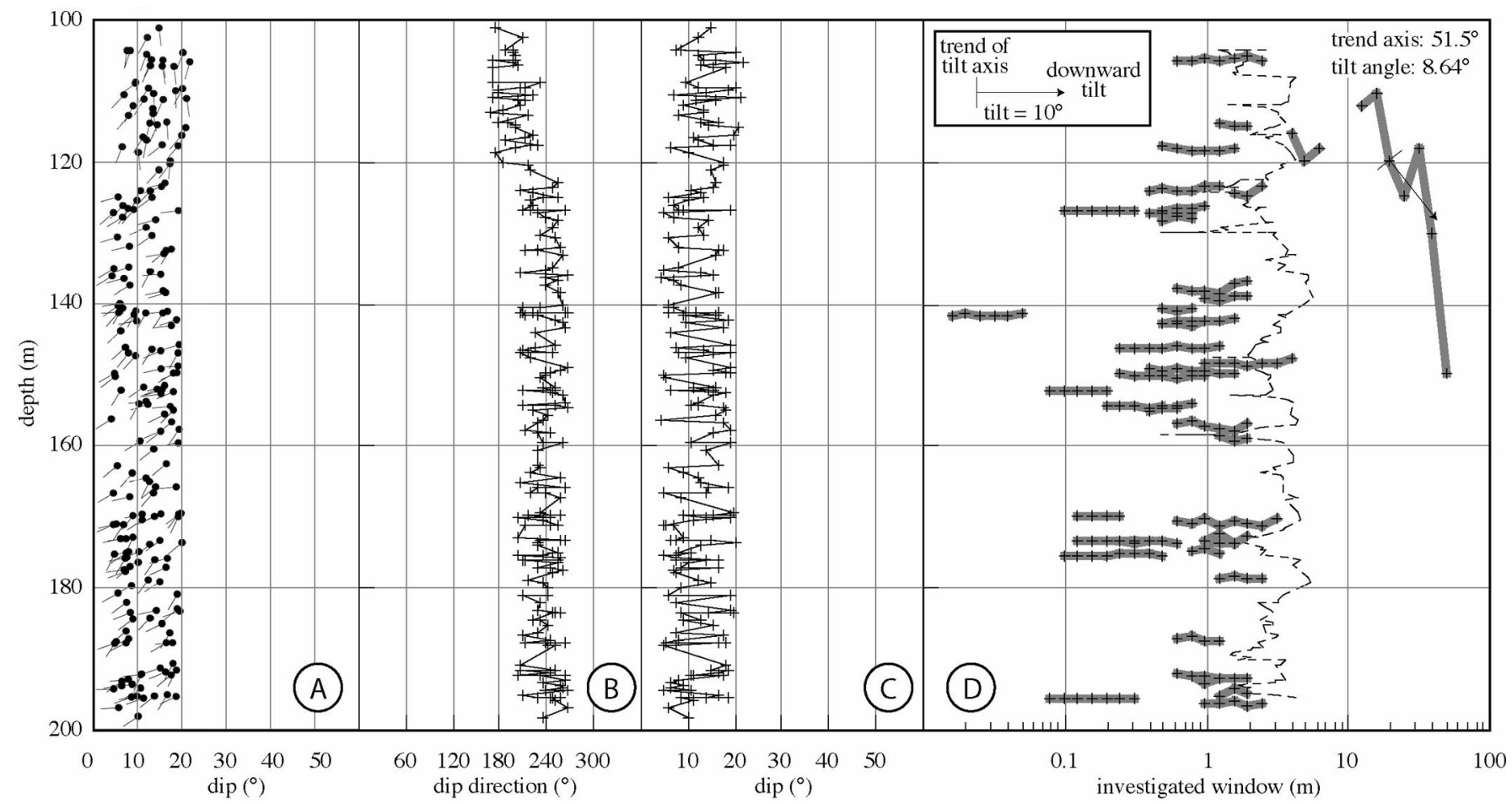

Figure 5 : Test3: Display of data and TrackDip results. Same legend as for Figure 4 . Cut-offs are $3^{\circ}$ for the rotation angle and $\pm 20^{\circ}$ for the rotation axis trend. See text for comments. 


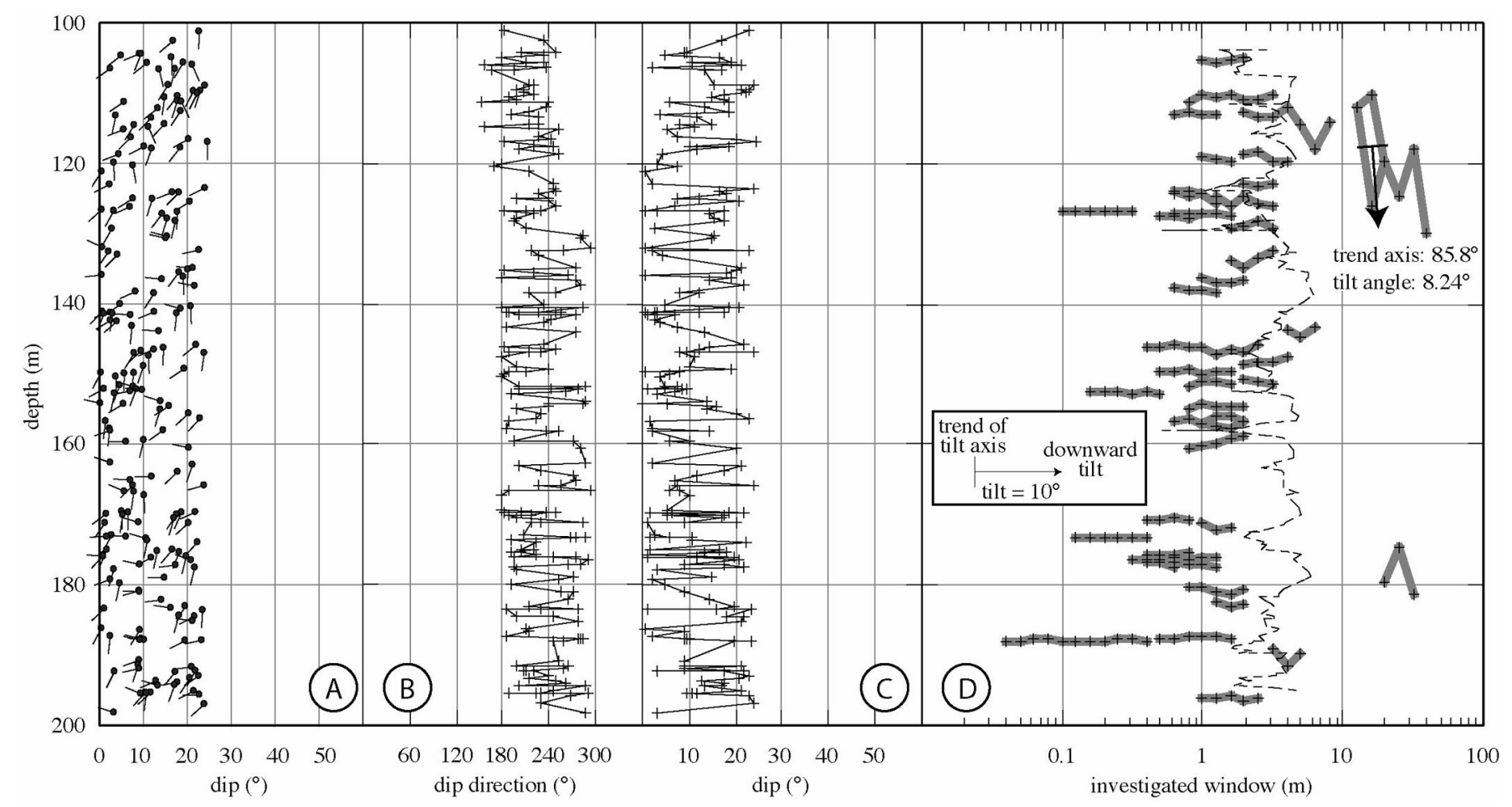

Figure 6 : Test4: Display of data and TrackDip results. Same legend as for Figure 4 . Cut-offs are $3^{\circ}$ for the rotation angle and $\pm 20^{\circ}$ for the rotation axis trend. See text for comments.

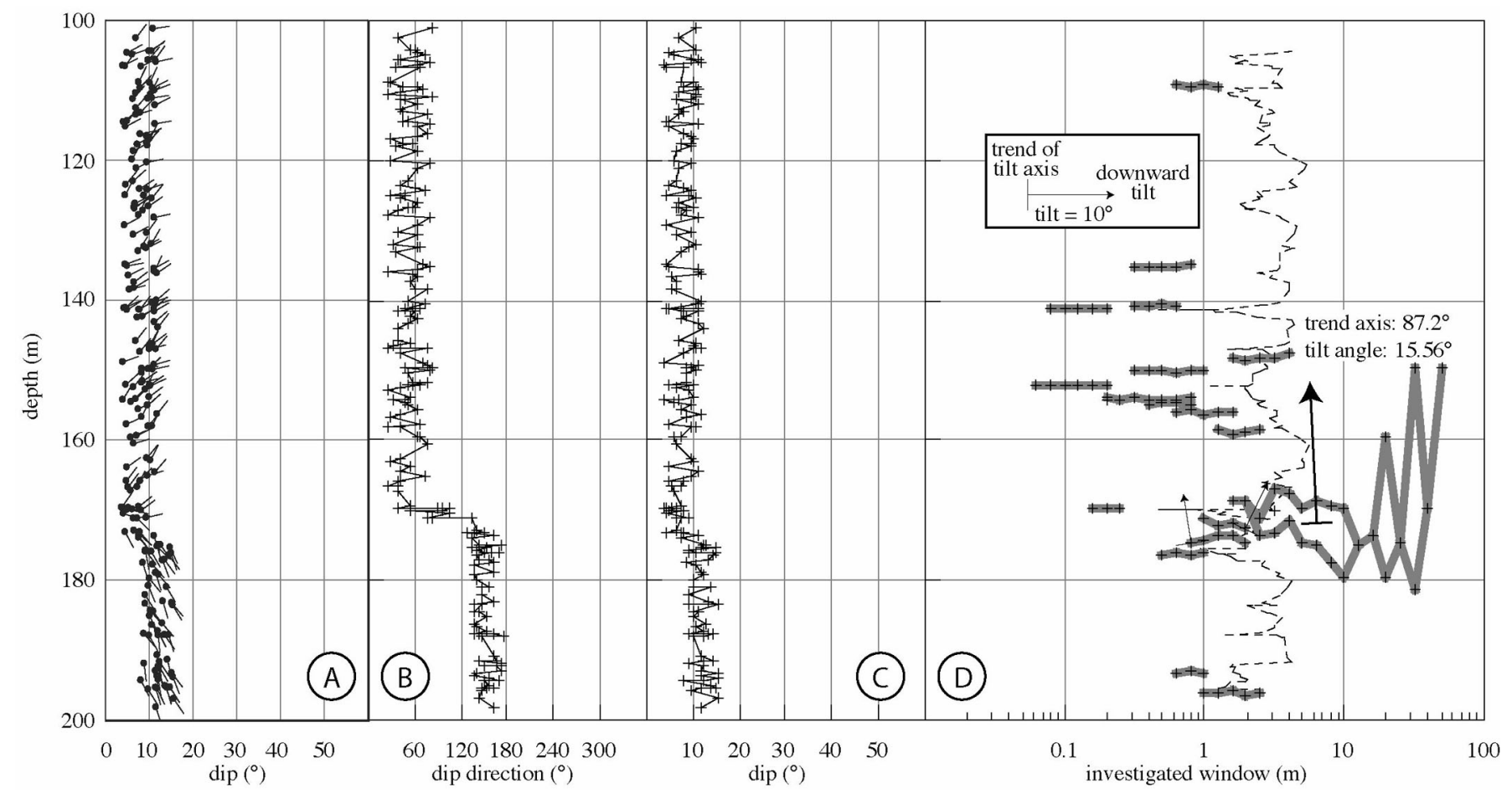

Figure 7 : Test5: Display of data and TrackDip results. Same legend as for Figure 4 . Cut-offs are $3^{\circ}$ for the rotation angle and $\pm 20^{\circ}$ for the rotation axis trend. See text for comments.

separated by several meters. Consequently, sampling rate is not constant, and the changes of attitude have to be studied at various scales.

We repeated some measurements ten times to estimate instrumental errors on azimuths and dips. The average standard deviations are $2.5^{\circ}$ on azimuth measurements (between $1.25^{\circ}$ and $3.34^{\circ}$ ), and $3.7^{\circ}$ on dip measurements (between $2.13^{\circ}$ and $5.09^{\circ}$ ). These errors are clearly smaller than the observed variations of azimuths (more than $30^{\circ}$ ) and dips (more than $40^{\circ}$ ) (Figures 15 to 17 ).

Section I (Figure 15) is a 74 m-high E-W section located just North of barranco Solano (Figure 11). The two other Sections are located $400 \mathrm{~m}$ North of Section I (Figure 11), and define two perpendicular sections across a same hill, N-S and E-W for Sections II and III, respectively (Figures 16 and 17). These two sections are close one to another, and several strata (A to D in Figures 16 and 17) can be 
Marine \& Petroleum Geology, accepted

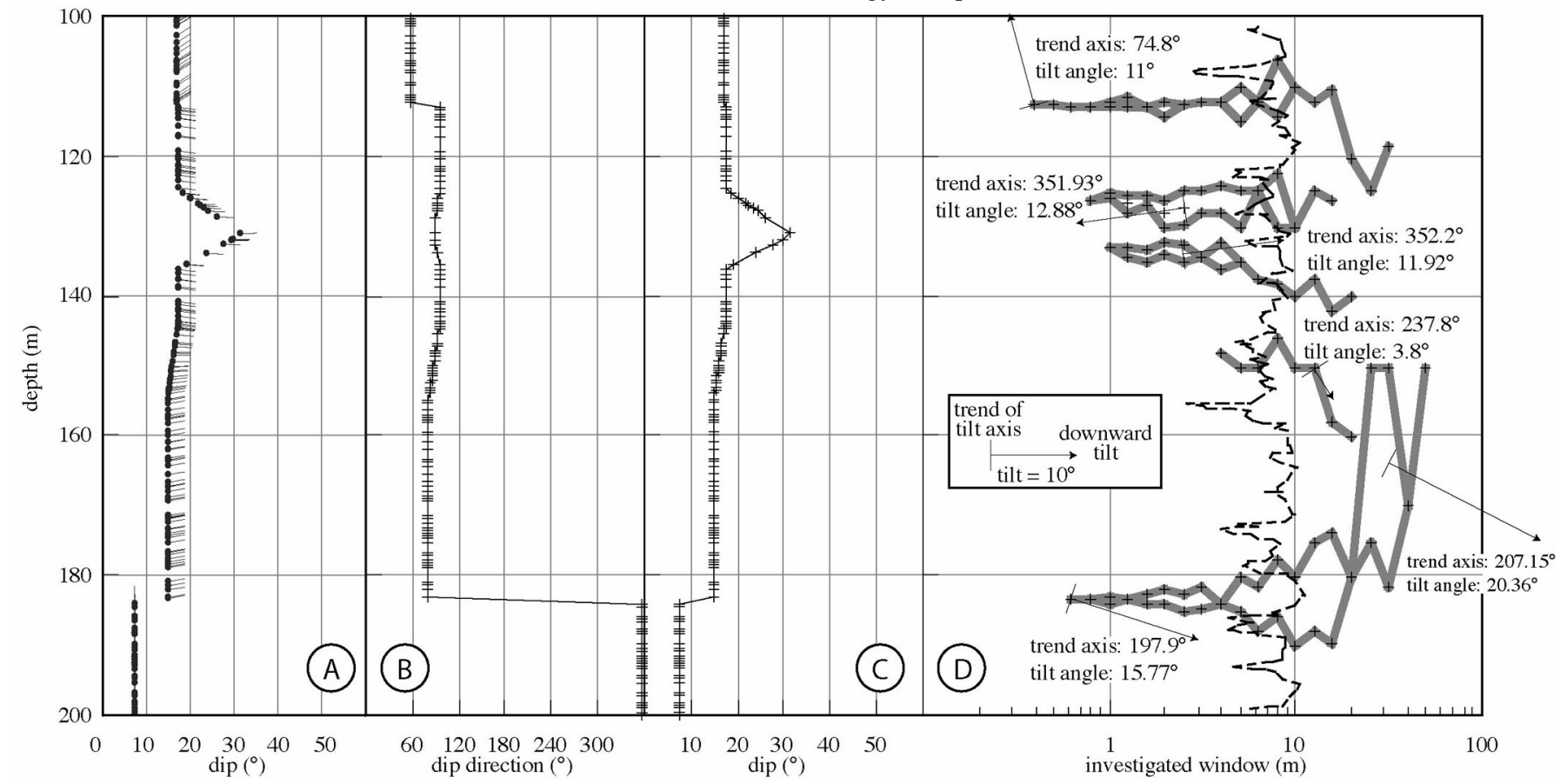

Figure 8 : Test6: Display of data and TrackDip results. Same legend as for Figure 4 . Cut-offs are $2^{\circ}$ for the rotation angle and $\pm 30^{\circ}$ for the rotation axis trend. See text for comments.

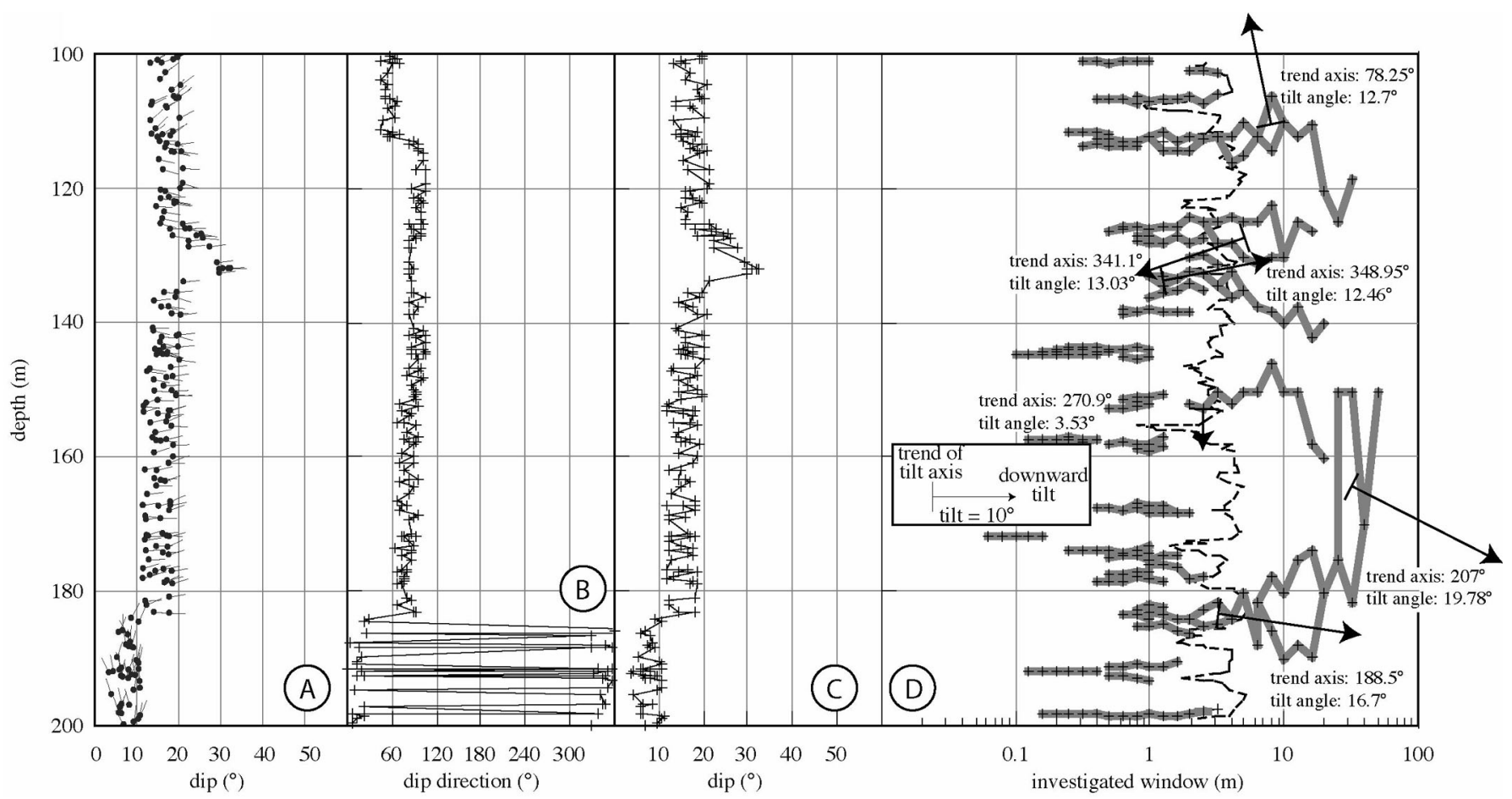

Figure 9 : Test7: Display of data and TrackDip results. Same legend as for Figure 4 . Cut-offs are $2^{\circ}$ for the rotation angle and $\pm 30^{\circ}$ for the rotation axis trend. See text for comments.

correlated. The two sections share the same measurements for strata $\mathrm{E}$ at the top of the hill. Above strata $\mathrm{C}$, the upper parts of Sections II and III are lateral equivalents of the first ten meters above the S3 unconformity located at 47.5 $\mathrm{m}$ in Section I.

In these three sections or in their vicinity, we observed several structures associated to changes of bed attitude (Figures 15 to 17): sedimentary structures are mainly cross-bedding in sandstones; syn-sedimentary or syn-diagenetic normal faults currently occur in mudstones. Some of these structures appear in three dimensions as decimetre- to meter-scale sliding scars. In Sections II and III, sedimentary dikes were also observed at the base of A sandstones, along S2 sliding surface (Figure 14). Finally, the large-scale changes of attitude are often associated to pinching-out beds, some of them clearly associated to the main unconformities $\mathrm{S} 1$ and $\mathrm{S} 3$ identified in the area (Callot et al., submitted) (Figures 12 and 13).

\subsection{Results of TrackDip process 4.3.1 Artefacts}


Marine \& Petroleum Geology, accepted

- The right parts of Figures 15 to 17 display the tilting events computed by TrackDip from the variations of bed attitude in Sections I to III, respectively (Annexes 3 and 4).

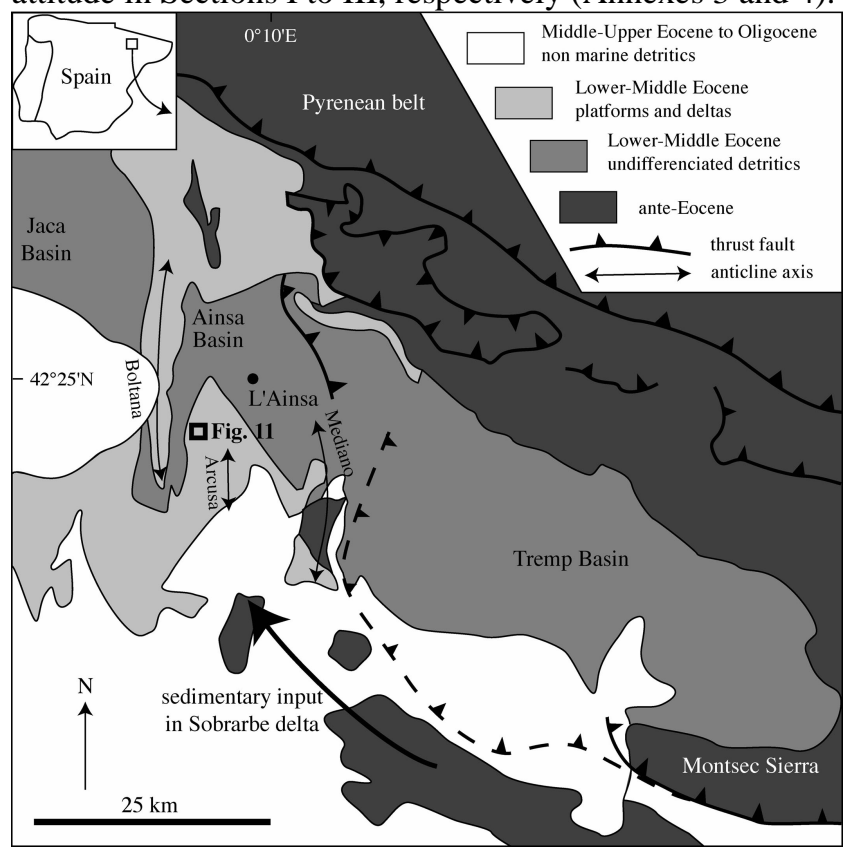

Figure 10: Simplified geological map of the central part of the spanish Eocene foreland basin (modified from Fernández Bellon, 2004). The box locates Figure 11.

We refer to these rotations by the Section number followed by the tilt number (e.g. I-7). In the three cases, the characteristic scales of identified rotations are of course constrained by the distance between consecutive measurements: in mudstones where bedding is sparse, characteristic scales vary from 1 to 10 meter, while they can be as small as $10 \mathrm{~cm}$ in sandstones. With this restriction, the dipmeter logs appear as a succession of tilts at various scales. However, some of these tilts are clearly related one to another. We exemplify these cases in Section I (Figure 15), but they were observed similarly in the two other sections :

- In some cases like I-69 and I-152 (elevation $7 \mathrm{~m}$ ), or I193 and I-229 (elevation $24 \mathrm{~m}$ ), TrackDip identifies two similar rotations at different scales but for the same elevations. As in Test2 (see above), this indicates an interruption of tilt tracking, due to too restrictive cut-offs. However, these two rotations correspond to a single tilt event which location is best indicated by the smallest window size.

- A peculiar feature occurs when the tilt axis progressively rotates when the window size decreases, such as for I-7, I-13 and I-48 (elevation 24 to $30 \mathrm{~m}$ ). This indicates a progressive change of preferred orientation with scale : smallest scales $(<1 \mathrm{~m})$ correspond to N-S trending rotation, while large scales correspond to E-W trending rotation. As in Test6 or Test7, this change occurs because large windows merge several individual tilts that occur at smaller scales.

\subsubsection{Section I : various scales}

Taking apart the variations associated to these artefacts, the tilts of Section I can be sorted as follow (Figure 15) :

At scales larger than $25 \mathrm{~m}$, two perpendicular tilts are observed. Both tilt ways indicate eastward dips in the lower part of the section, consistent with the decrease of dip with elevation in the section. However, tilt trends differ between the lower part of the section (I-1, trending $\mathrm{N} 30^{\circ}$ ) and the upper one $\left(\mathrm{I}-2\right.$, trending $\left.\mathrm{N} 140^{\circ}\right)$. I-2 rotation indicates a progressive tilt from 46 to $76 \mathrm{~m}$, which is better defined at a 10 -meters scale.

At 10-meters scale, we observe three successive tilts : below $25 \mathrm{~m}$, two tilts (I-9 and I-3) with similar trend $\left(\mathrm{N} 160^{\circ}\right)$ but opposite ways; in the 20-35 m-interval, southward tilt I-7 is E-W-trending ; finally in the upper part of the Section (above $35 \mathrm{~m}$ ), N140 ${ }^{\circ}$-trending tilts occur with alternative tilt ways (I-10, I-6, I-2).

At smaller scales, the variability of the intervals between consecutive measurements precludes a description of consecutive tilts. However, we observe only few preferred tilt orientations: in the lowest part of the Section, two consecutive and opposite tilts (I-15 and I-124) trend NE$\mathrm{SW}$ at few meters to few decimetres scales. Elsewhere, N-S trending opposite tilts prevail at the smallest scales, including less than $10 \mathrm{~cm}$-scales. Most of

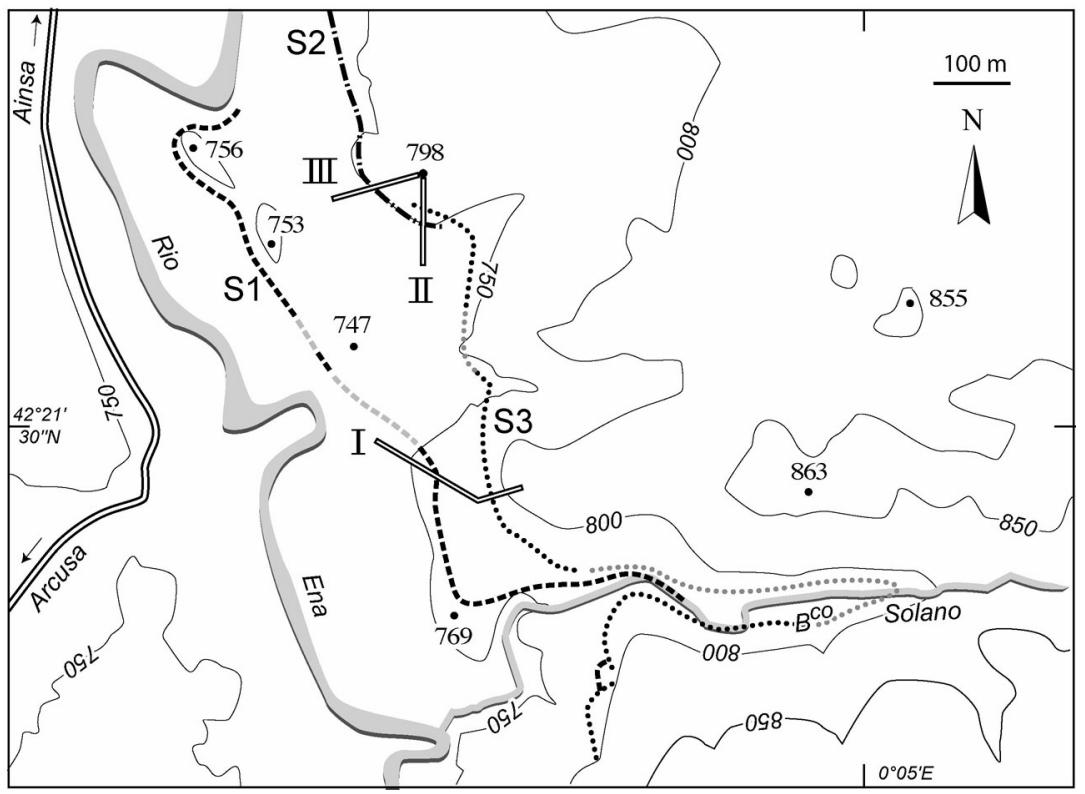


Figure 11 : Location of studied Sections I to III (Figures 12, 13, 15 to 17) and main sliding structures (S1 to S3, according to Callot et al., submitted). S1 and S3 in grey where hidden. Elevation in meters.

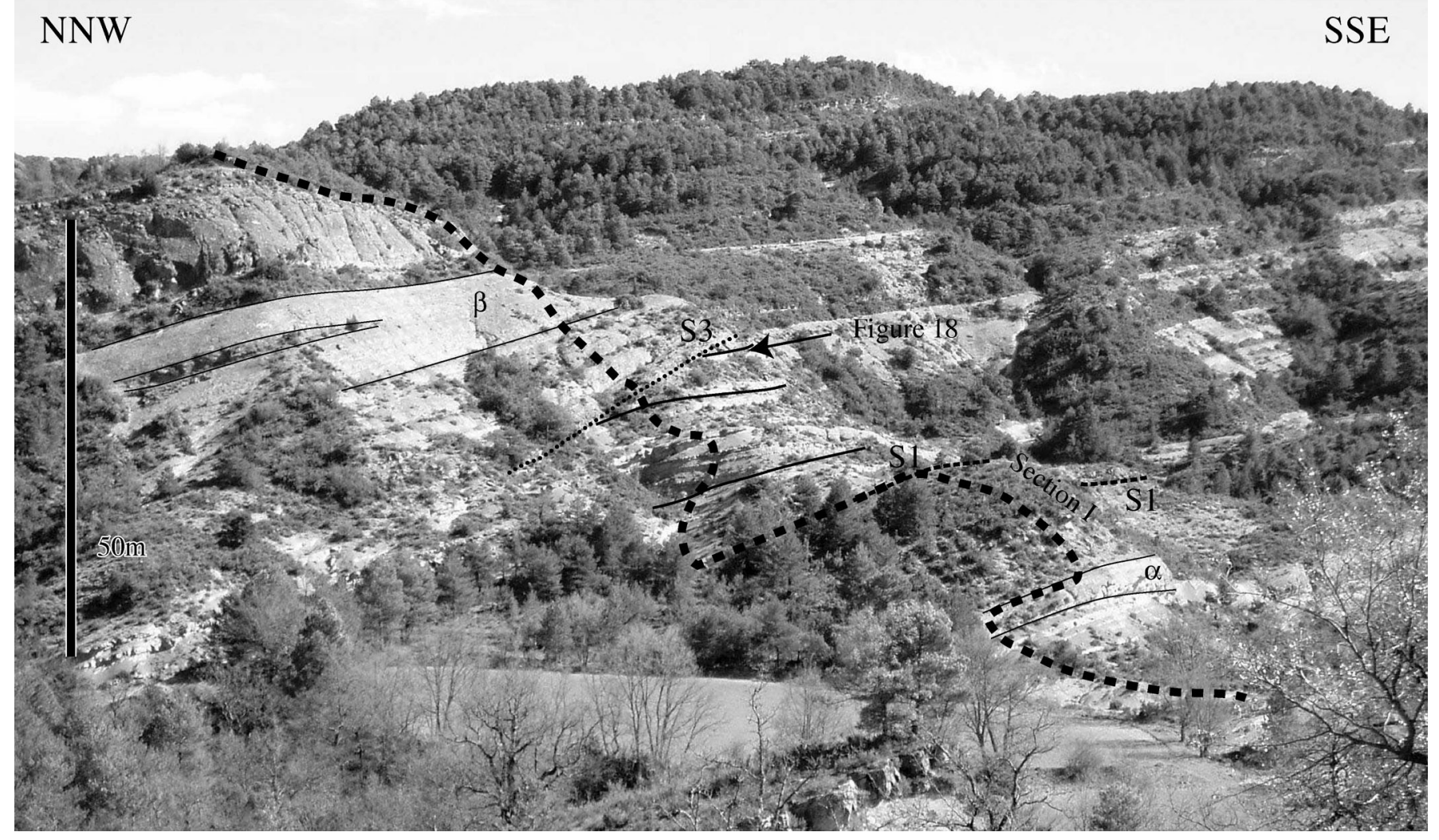

Figure 12: Section I outcrop, located on Figure 11. Dipmeter measurements follow the dashed line; the lowest part of the section is not visible on this picture. The main sliding surfaces S1 and S3 are underlined, together with stratigraphic markers $\alpha$ and $\beta$ (Figure 15). View to the south east.

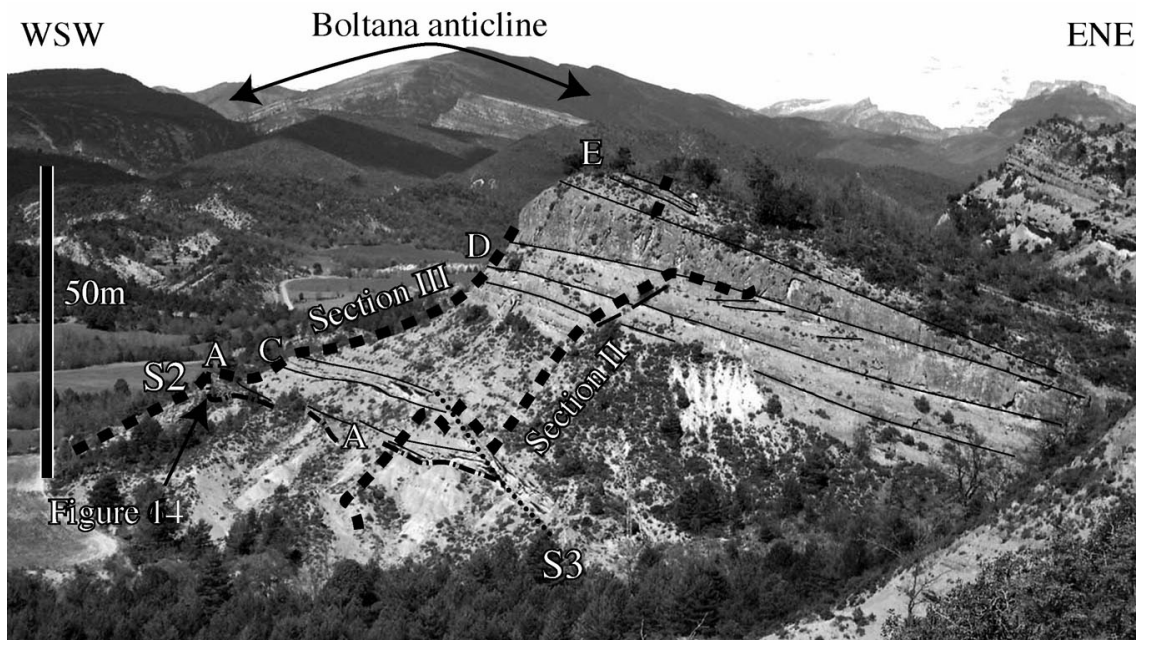

Figure 13 : Sections II and III outcrop, located on Figure 11. Dipmeter measurements follow the dashed lines. The main sliding surfaces S2 and S3 are underlined, together with some stratigraphic markers (A, C to E, Figures 16 and 17). Notice the changes of thickness between D and E. View to the north, Boltana anticline in the background.

these rotations are first (from bottom to top) eastward tilts, then westward (e.g. I-34, I-152 and I-69 followed upward by I-43, I-9 and I-27). E-W trending tilt axes appear in the 25 to $48 \mathrm{~m}$ interval (e.g. I-63 at $41 \mathrm{~m}$ or I-22 at $47 \mathrm{~m}$ ). Finally, many successive tilts indicate similar rotation axis and angle, but opposite tilt ways (e.g. I-15 and I-124, elevation 2 to $5 \mathrm{~m} ; \mathrm{I}-27$ and I-3, elevation 12 to $15 \mathrm{~m} ; \mathrm{I}-10$ and I-6, elevation 38 to $46 \mathrm{~m}$ ). These pairs indicate that two sub-parallel beds enclose oblique beds, as cross-bedding between horizontal beddings (Figure 2A).

4.3.3 Section I : comparison with field observations

At all scales, several identified tilts perfectly fit the structures observed on the field :

In the sandstones, the observed cross-bedding (23, 24, 48 $\mathrm{m}$ in Section I) are dune-like structures, with oblique beds 
dipping East between paleo-horizontal surfaces (e.g. Figure 18). These structures fit the identified N-S trending tilts at the same elevations (I-143, I-193 and I-229, I-223, I-204, I212). Tilts with similar trends are also identified in other sandstone beds where cross-bedding was not directly observed (I-152, I-69, I-43, I-9 in the 7-10 m interval;

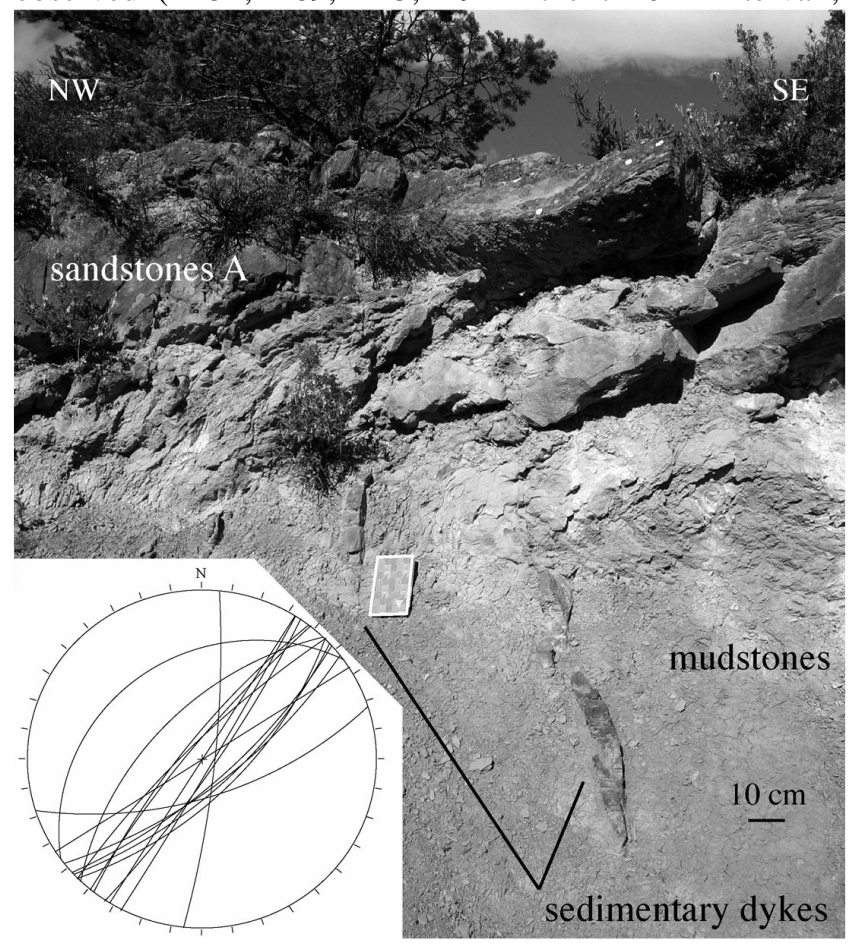

Figure 14 : Sedimentary dykes in the mudstones below sandstones A and sliding surface S2 (located in Sections II and III, Figures 16 and 17). Stereographic projection (Wulff net, lower hemisphere) of the dykes measured in the same stratigraphic layer. Notebook $(17.5$ x $11.5 \mathrm{~cm})$ for scale. Location Figure 13 .

$\mathrm{I}-245$ and $\mathrm{I}-157$ in the $25-27 \mathrm{~m}$ interval). All these observations indicate a westward transport of detritic particles in this part of the delta.

At a larger scale, nummulitic limestones are systematically associated to NNW-SSE trending rotations with an ENE tilt way (I-3 at $14 \mathrm{~m}, \mathrm{I}-2$ in the uppermost part of Section I).

Finally, pinching out and unconformities are wellidentified: S1 and S3 sliding surfaces are similarly identified by E-W-trending tilts (I-49 and I-109, and I-22, respectively), with the underlying beds truncated by a north-dipping unconformity. The same E-W-trending tilt (I-63 at $41 \mathrm{~m}$ ) is observed associated to pinching out at a smaller scale between the two surfaces. However, smallscale and large-scale pinching out overlying S1 and S3 are mainly characterized by similar NW-SE-trending tilts : tilt $\mathrm{I}-10$ fits the $38-40 \mathrm{~m}$ pinching out associated to $\mathrm{S} 1$ unconformity, with similar NW-SE trend and tilt way as for rotations I-3 $(10-20 \mathrm{~m})$ and I-2 $(46-74 \mathrm{~m})$, which represents the large-scale wedge overlying S3 unconformity (Figure 12). I-9 (10 m) and I-6 (45 m) share the same NW-SE axis but with an opposite tilt way.

On the contrary relationships between identified tilts and small-scale paleo-scars are not obvious : the trends of rotation axis do not clearly fit the orientation of sliding surfaces (Figure 15) : I-34 at $6 \mathrm{~m}$ trends $\mathrm{N} 25^{\circ}$ while the sliding surfaces strike $\mathrm{N} 125^{\circ}$ to $\mathrm{N}-\mathrm{S}$; I-72 at $18 \mathrm{~m}$ trends $\mathrm{N} 145^{\circ}$ while the sliding surfaces strike $\mathrm{N}-\mathrm{S}$ and $\mathrm{N} 75^{\circ}$. At
$24 \mathrm{~m}$, the sliding surfaces strike N-S, parallel to many N-S trending rotations that can also be attributed to crossbedding. At $37 \mathrm{~m}, \mathrm{I}-101$ trends $\mathrm{N}-\mathrm{S}$ as sliding surfaces observed laterally.

\subsubsection{Sections II and III}

In lower parts of Sections II and III (Figures 16 and 17), the large scales $(>7 \mathrm{~m}$ ) indicate tilts trending NE-SW (II-8 and II-12) and N-S (III-11, III-1 and III-12), respectively. Similar NW-SE-trending tilts occur in the upper parts of both sections (II-13, II-15, II-3 ; III-13, III-8, III-5).

As in Section I, numerous structures observed on the field are directly identified by significant tilts. In the sandstones, tilts in the observed cross-bedding are still trending N-S (III-72 and III-141, III-41 and III-90 in the 11-15 m interval) or NNW-SSE (E strata in Sections II and III). Several tilts fit the location and orientation of the observed small-scale paleo-scars (III-67, III-44 and III-13, III-8 at 22, 26 and $37 \mathrm{~m}$, respectively) or normal faults (III53 at $1 \mathrm{~m}$ ), while others (II-15 and II-29, II-24 at 30 and 34 $\mathrm{m})$ are oblique or perpendicular to the orientation of paleoscars. The sedimentary dykes striking $\mathrm{N} 40^{\circ}$ (Figure 14) that underlie S2 surface are also well-recorded in both sections (II-44; III-26, III-71 and III-88). Finally, the unconformities located at 9 and $11 \mathrm{~m}$ (S3) in Section II correspond to E-W-trending tilts (II-45, II-22 and II-37, II27 and II-38).

As the same data set has been used in the uppermost part of Sections II and III (strata E), it represents a good test to investigate how the change of position of the investigated windows (which is linked to the depth frame) influences the identification of significant tilts : because the position of the investigated windows differs in the two sections, the tracked tilts are not exactly identical. However, both Sections display a similar succession of tilts, for the same strata, with similar trends, angles and tilt ways. Tilt II-119 is the only exception, as it is not observed in Section III, where the change of position of the investigated windows makes the rotation angle too small to be retained.

\subsection{Interpretation}

Dipmeter processing in the three investigated sections shows that this method is able to detect, localize and measure the sedimentary (cross-bedding, unconformity) or tectonic structures (sliding structure, scar, normal fault) observed on the field at various scales. Obviously, this identification does not allow directly identifying and naming each type of structure, but provides information on the geometry of the structures, and allows discriminating by their orientations structures that can appear comparable on the field. Furthermore, it appears that even when no peculiar structures were observed, the bed attitudes do not vary randomly, but their tilts have preferred orientations that can be ascribed to specific processes identified on other parts of the section. Finally, there are some evidences of reproducibility: in the studied sections we observe similar evolution for two close sections (II and III), especially where the lateral continuity is clear (between strata D and E), but also in the upper part of Section I. Because of shift in the position of investigated windows, there are only slight changes in the results for strata $E$ between Sections II and III. 
Marine \& Petroleum Geology, accepted

Of course, dipmetry processing can not be used alone, as it does not provide by itself a geological interpretation of the changes of orientation. But the fact that these changes of orientation are specific to some scales makes the comparison with other data easier. On the field, this method can be time-consuming (for systematic measurements along a section), and lacking precision (because of compass measurements). On the contrary, in

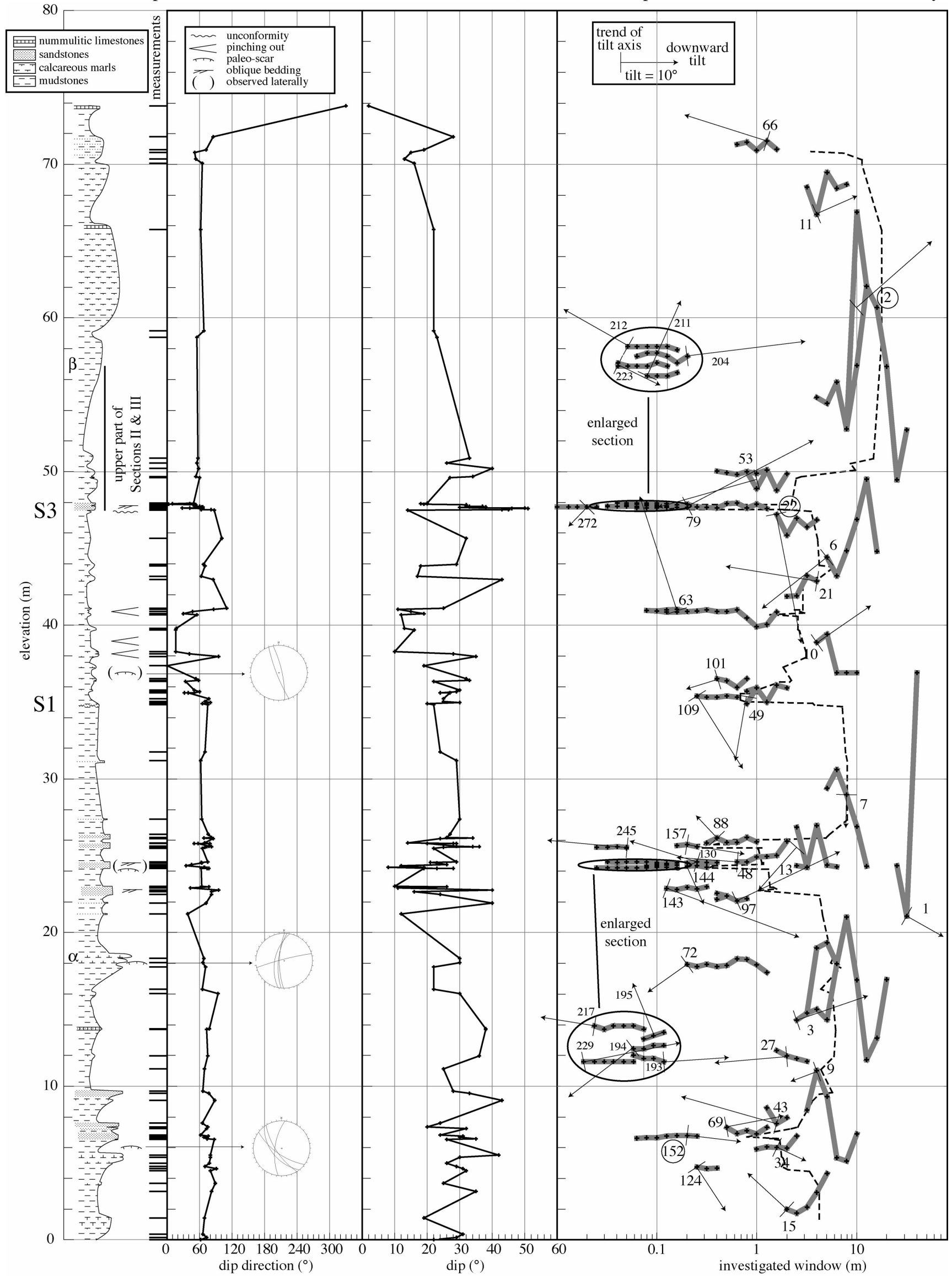

Figure 15 : Sedimentary and dipmeter (dip direction and dip) logs for Section I. Strata $\alpha$ and $\beta$ and sliding surfaces S1 and S3 are located on Figure $12 ; \mathrm{S} 1$ and S3 are also mapped on Figure 11. The lateral equivalent of the upper part of Sections II (above S3) and III (above layer C) 
Marine \& Petroleum Geology, accepted

is located above S3 surface. On the righten part, tilt tracking (grey lines) as a function of investigated window size, and identified significant tilts from processing of dipmeter data (cf. text and Figure 1). The dotted line indicates the size of the windows containing seven data. Tilts are referenced by a number attributed during tracking. They are referenced in the text by the Section number and the tilt number (e.g. I-7) ; circled numbers exemplify the structures drawn in Figure 19. Stereographic projections : Wulff nets, lower hemisphere.

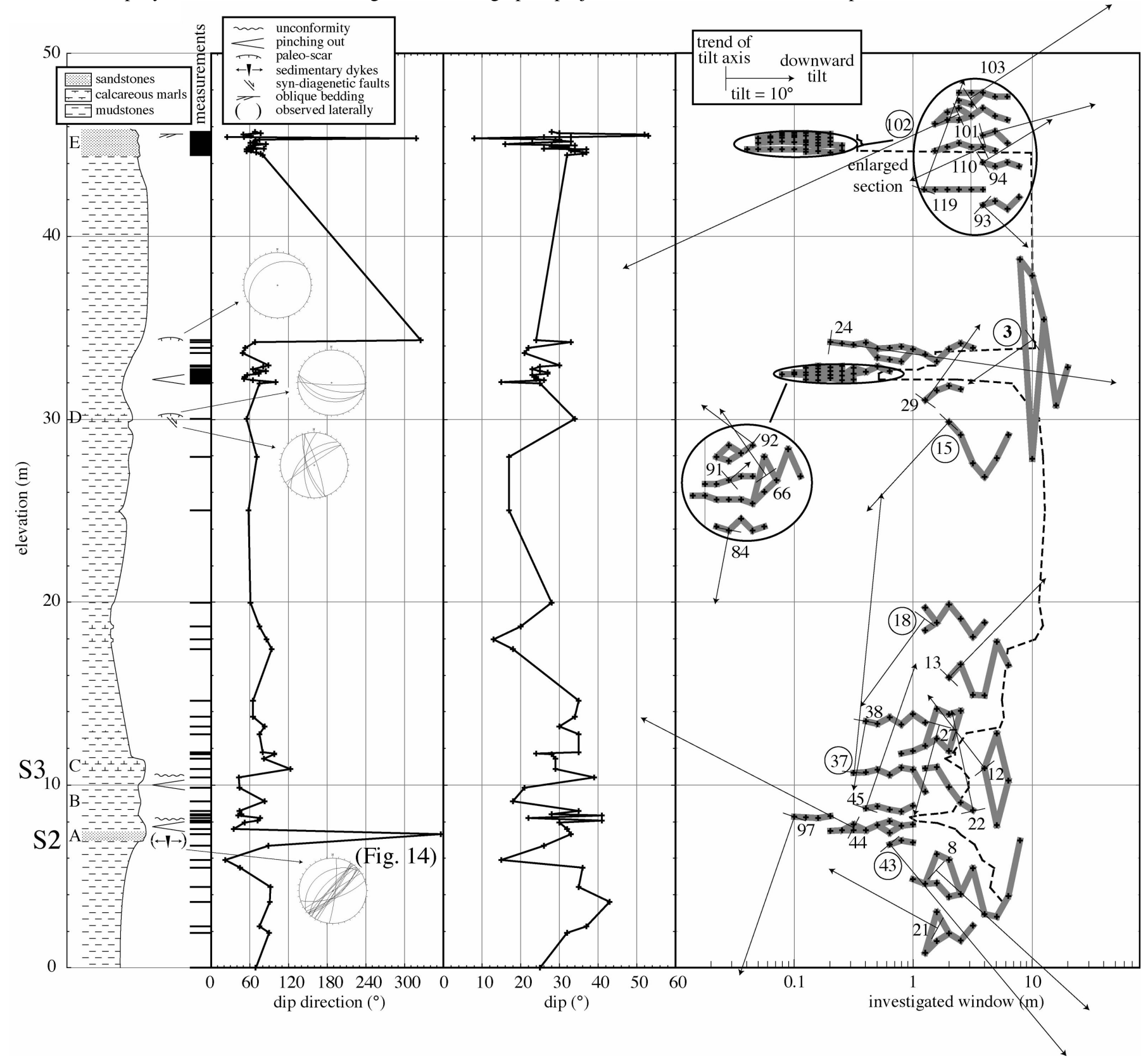

Figure 16 : Section II. A to E are common stratigraphic markers in Sections II and III. Strata A and C to E and sliding surfaces S2 and S3 are located on Figure $13 ;$ S2 and S3 are also mapped on Figure 11. Same caption as for Figure 15.

drilled borehole, as dipmeter tools provide fast and precise measurements, and moreover allow a systematic survey of bedding attitudes in thicker sedimentary sections, the processing method used herein may be a useful tool to integrate and interpret structural measurements with the other logging records.

For the specific study of the gravity-driven structures in the Sobrarbe delta, the dipmetry results can be interpreted quite simply as a combined effect of sedimentary inputs and large scale tectonic deformations. In the three studied sections as in adjacent places, all evidences, either direct (dunes, cross-bedding, flute-casts) or from dipmetry analysis (N-S-trending tilts in sandstones: I-152, II-102, III-72...), indicate a westward clastic transport. There are only small-scale evidences of N-S-trending gravity-driven sliding (e.g. III-13 and III-44) that were probably controlled by sedimentary overloading. These sediments deposited north of the northern termination of the N-Strending Arcusa anticline, and west of northern termination of Mediano anticline (Dreyer et al., 1999; Figure 19). While detrital displacement was controlled southward by the N-S-trending Mediano and Arcusa anticlines, the westward transport in the studied area probably indicates a change of orientation of the transport network where the Arcusa anticline disappears (Figure 19). An alternative hypothesis can be an independent detrital network coming from the uplifted westernmost part of Tremp basin (Figure 5 in Dreyer et al., 1999), crossing the northern termination of Mediano anticline, and converging with the main delta north of Arcusa anticline (Figure 19). 
Marine \& Petroleum Geology, accepted

The large scale tectonic deformations are best indicated by E-W-trending tilt axis, associated to S1 and S3 unconformities in Sections I and II (I-49, I-22 and II-37). These slides can be interpreted either as resulting from the regional northward tilt of the flexural basin, or more probably as the southward uplift of the growing Arcusa anticline (Figure 19). For these two sliding surfaces, the recorded tilts in the studied sections are by several orders

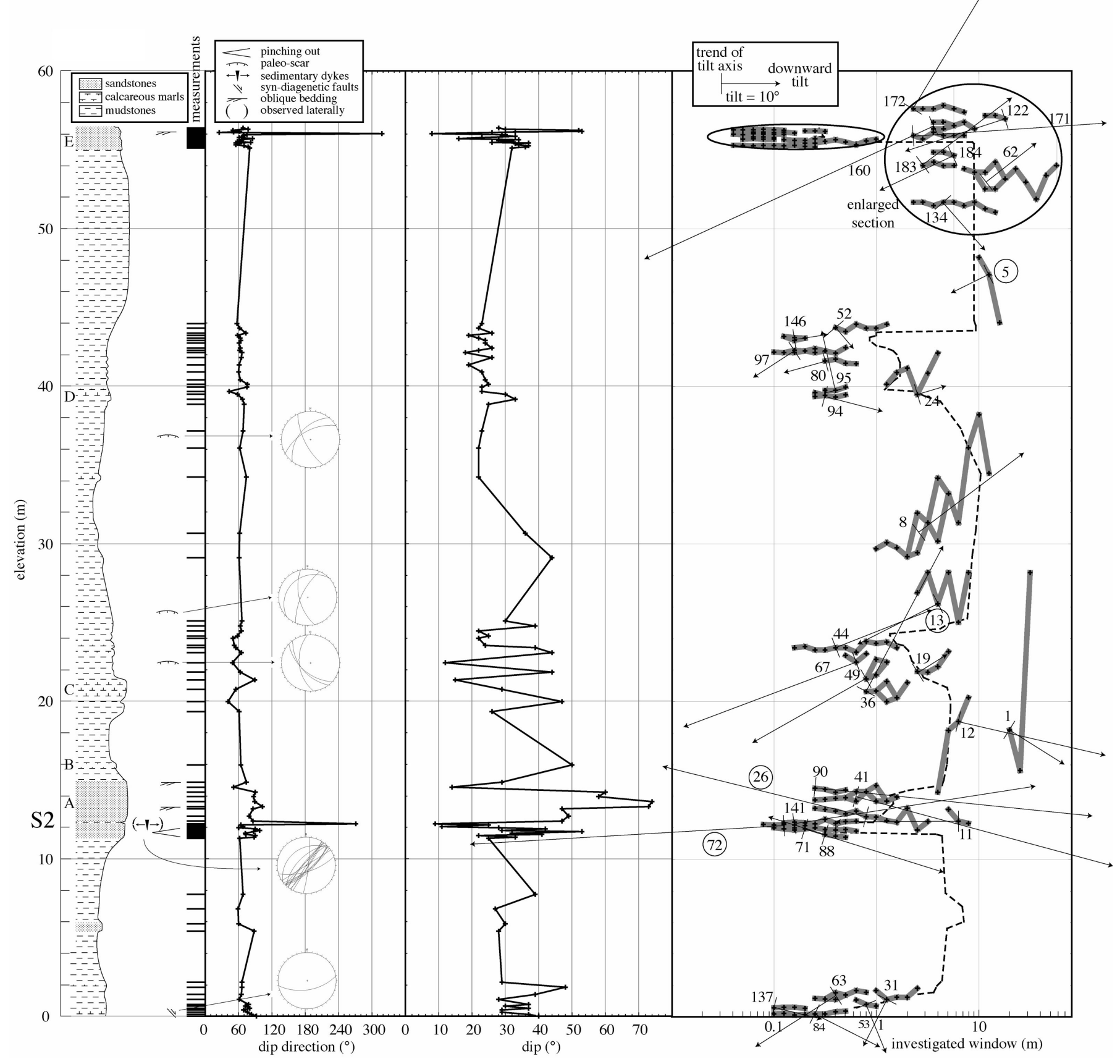

Figure 17 : Section III. A to E are common stratigraphic markers in Sections II and III. Strata A and C to E and sliding surface S2 are located on Figure 13 ; S2 and S3 are also mapped on Figure 11. Same caption as for Figure 15.

larger than the regional tilt that can be induced by folding or foreland basin flexure: these mechanisms can trigger gravity-driven instabilities, inducing locally steep slopes.

A combination of this northward regional tilt with a westward sedimentary transport may generate a NE-SW striking slope at the edge of the delta. This NE-SW direction is mainly recorded by large scale tilts in the lower parts of Sections I and II, but also by extensional structures (normal faults, some small-scale scars, sedimentary dykes), especially associated to sliding surface S2 in Sections II and III (Figure 19).

Finally, it has been observed a significant, while not systematic, difference between tilt axis trend and scar strikes. As proposed by Callot et al. (submitted), this difference may be due to the curvature of slump scar. The arcuate fault may range from perpendicular to the sliding displacement (in the centre) to parallel to it (on the edges). A good example is given by the structures observed at 30 $\mathrm{m}$ in Section II (Figure 16), where normal faults accommodates a WNW-ESE extension, but where the overlying sliding scars observed just above strike E-W, and probably represent the edge of an arcuate structure. In this case as at larger scale in the upper part of the three Sections, the associated NNW-SSE-trending tilt axis can represent the structures associated to the edge of NNWSSE elongated sliding surfaces S1 and S3 (Figure 19). 
Marine \& Petroleum Geology, accepted

Furthermore, the large-scale NNW-SSE-trending tilt associated with nummulitic limestones in Section I, and it infilling from the side the depressions formed by previous ENE tilt way can be interpreted as the record of the southwestward progradation of the overlying mudstones,

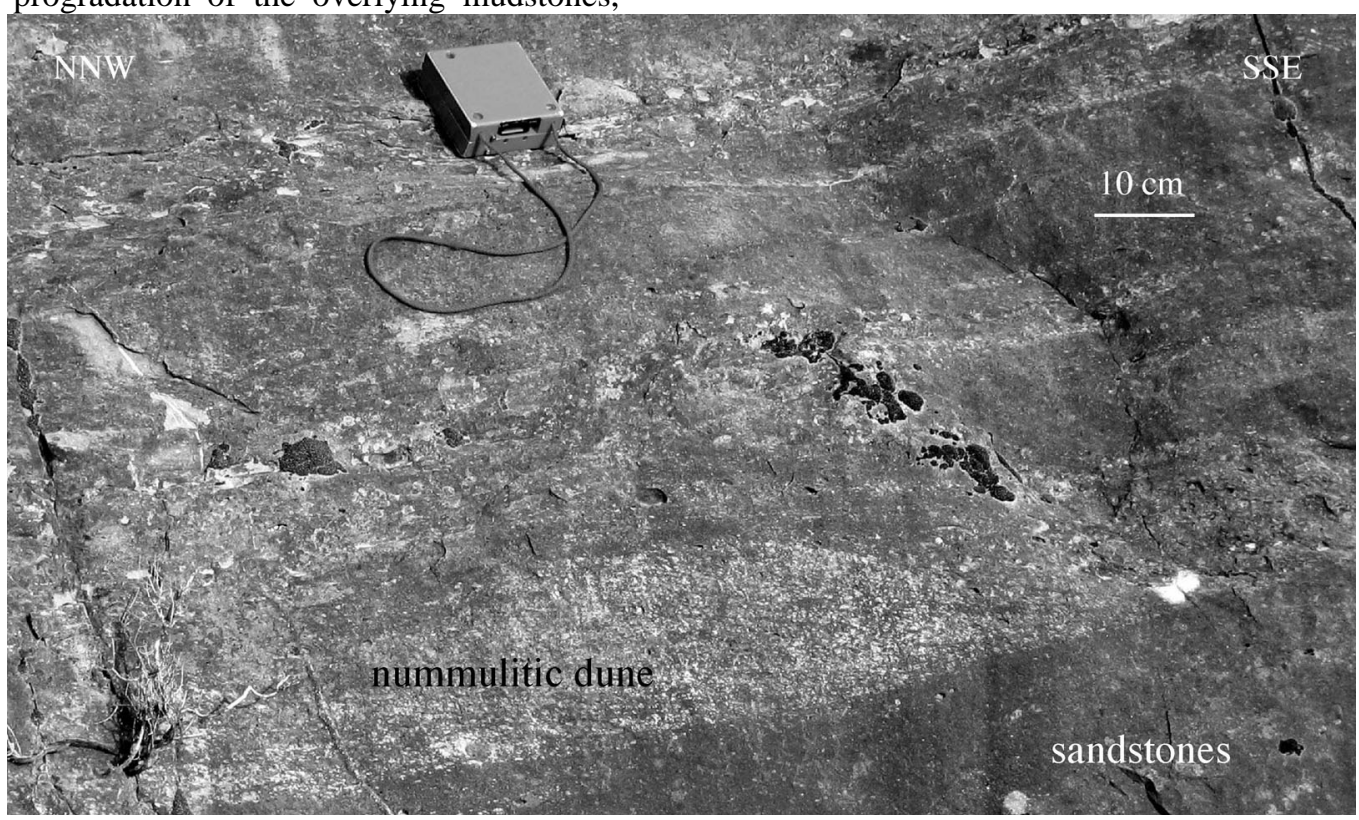

Figure 18 : Submarine dune built by an accumulation of Nummulites (white dots). Lateral equivalent of sandstones, elevation $48 \mathrm{~m}$, Section I (Figure 15). Compass for scale (9 x $10 \mathrm{~cm})$. Location Figure 12.

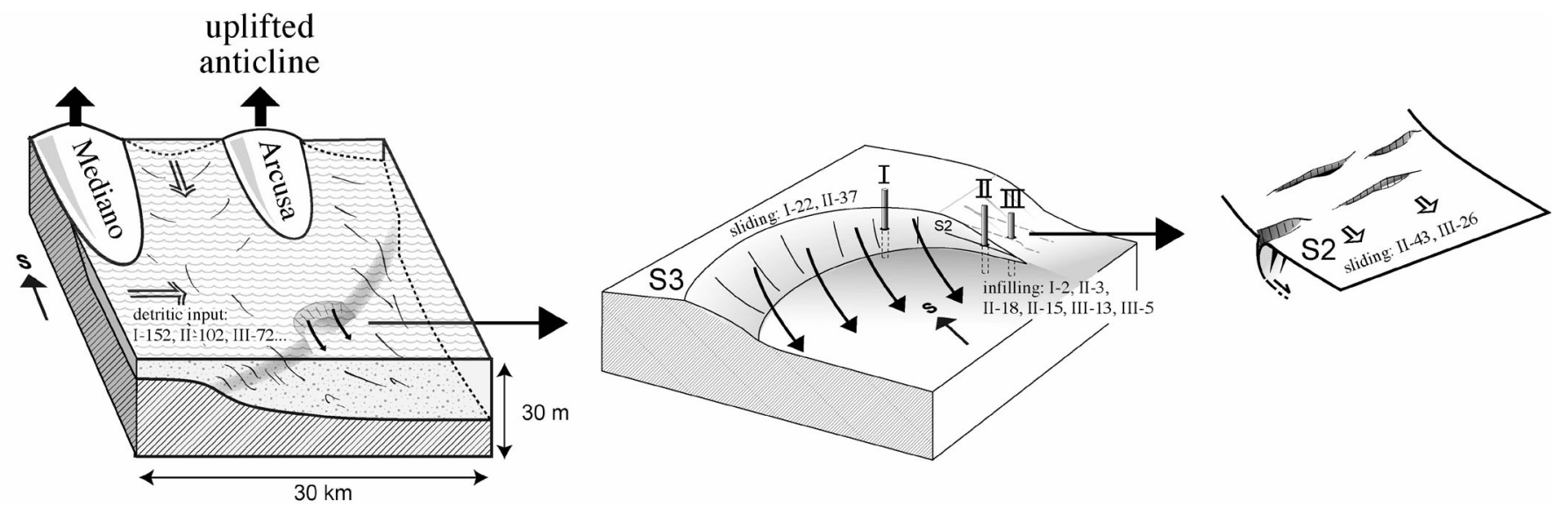

Figure 19: Schematic 3-D view of the main types of structures observed and deduced from dipmeter logs. Numbers refer to examples of tilts associated to each type of structure.

\section{Conclusions}

While needing a careful interpretation, the proposed TrackDip processing method of dipmeter data appears to be able to detect, localize and measure the structures independently observed on the field. Because this method integrates several scales, it provides additional information that can not be available from the dipmeter measurements alone. Easier applications of TrackDip can be expected from dipmetry logging in boreholes, even if the geological interpretation will need in every case an integration of all available data. For gravity-driven slides this method can be helpful, as tilt trends can be expected to be close to the perpendicular of the slide directions, while fault strikes can not easily be related to displacements in arcuate structures.

In the studied area, the variations of orientation of the sedimentary beds can be interpreted as resulting from a sedimentary input from east to west, regional tilts towards North triggering the main gravitational instabilities, and a NE-SW local submarine slope.

\section{Acknowledgments}

This work has been funded by the french Groupes De Recherche 'Marges' and 'Corinthe'. We thank our colleagues from the GDR Marges group 'gravity instabilities', especially for discussions during the Toulouse workshop in 2004. We are particularly grateful to J. Lofi and P. Marza for their reviews and comments, and to V. Gaullier for her editorial work. 
Marine \& Petroleum Geology, accepted

Basile, C., 2000. Late Jurassic sedimentation and deformation in the west Iberia continental margin : insights from FMS data, ODP Leg 173. Marine and Petroleum Geology, 17, 709-721.

Bengtson, C. A., 1981. Statistical curvature analysis techniques for structural interpretation of dipmeter data. American Association of Petroleum Geologists Bulletin, 65, 312-332.

Berg, C. R., 1998. Synthetic deviation: a new dipmeter interpretation method. American Association of Petroleum Geologists Bulletin, 82, 6, 1133-1139.

Callot, P., Odonne, F., Debroas, E. J., Maillard, A., Dhont, D., Basile, C., Hoareau, G. 3D architecture and soft sediment deformations of submarine slide surfaces in the Eocene Sobrarbe deltaic complex (Ainsa, Spanish Pyrenees). Sedimentology, submitted (December 2007).

Donselaar, M.E., Schmidt, J.M., 2005. Integration of outcrop and borehole image $\operatorname{logs}$ for high-resolution facies interpretation : example from a fluvial fan in the Ebro Basin, Spain. Sedimentology, 52, 1021-1042.

Dreyer, T., Corregidor, J., Arbues, P., Puigdefabregas, C., 1999. Architecture of the tectonically influenced Sobrarbe deltaic complex in the Ainsa Basin, northern Spain. Sedimentary Geology, 127, 3-4, 127-169.

Fernández Bellon, O., 2004. Reconstruction of geological structures in 3D : an example from the southern Pyrenees. Ph-D Thesis, Universitat de Barcelona, 317 p.

Gilreath, J.A., 1987. Dipmeter interpretation rules - part 1. The Technical Review, 35, 3, 28-41.

Hesthammer, J., Fossen, H., 1998. The use of dipmeter data to constrain the structural geology of the Gullfaks Field, northern North Sea. Marine and Petroleum Geology, 15, 549-573.
Höcker, C., Eastwood, K. M., Herweijer, J. C., Adams, J. T., 1990. Use of dipmeter data in clastic sedimentological studies. American Association of Petroleum Geologists Bulletin, 74, 2, 105-118.

Hurley, N. F., 1994. Recognition of faults, unconformities, and sequence boundaries using cumulative dip plots. American Association of Petroleum Geologists Bulletin, 78, 8, 11731185 .

Luthi, S. M., Banavar, J. R., 1988. Application of borehole images to three-dimensional geometric modeling of eolian sandstone reservoirs, Permian Rotliegende, North Sea. American Association of Petroleum Geologists Bulletin, 72, 9, 1074-1089.

Puigdefabregas, C., Muñoz, J. A., Verges, J., 1991. Thrusting and foreland basin evolution in the southern Pyrenees. In : McClay, K. (Ed.), Thrust tectonics. Chapman and Hall, London, pp. 247-254.

Serra, O., 1989. Formation MicroScanner image interpretation. Schlumberger Educational Services, Houston, 117 p.

\section{$\underline{\text { Annexes }}$}

Annex 1 : Dipmetry measures (dip direction, dip, elevation) for Sections I to III.

Annex 2 : Computed tilts for Sections I to III.

Annex 3 : Significant tilts (displayed as paths in Figures 15 to 17) for Sections I to III.

Annex 4 : Retained tilts (displayed in Figures 15 to 17$)$ for Sections I to III. 


\begin{tabular}{|c|c|c|c|c|c|c|c|c|c|c|c|}
\hline \multicolumn{3}{|l|}{ Section I } & \multicolumn{3}{|c|}{ Section I (continued) } & \multicolumn{3}{|l|}{ Section II } & \multicolumn{3}{|l|}{ Section III } \\
\hline $\begin{array}{c}\text { dip direction } \\
\left({ }^{\circ}\right)\end{array}$ & $\begin{array}{l}\text { dip } \\
\left({ }^{\circ}\right)\end{array}$ & $\begin{array}{c}\text { elevation } \\
(\mathrm{m})\end{array}$ & $\begin{array}{c}\text { dip direction } \\
\left({ }^{\circ}\right)\end{array}$ & $\begin{array}{l}\text { dip } \\
\left({ }^{\circ}\right)\end{array}$ & $\begin{array}{c}\text { elevation } \\
(\mathrm{m})\end{array}$ & $\begin{array}{c}\text { dip direction } \\
\left({ }^{\circ}\right)\end{array}$ & $\begin{array}{l}\text { dip } \\
\left({ }^{\circ}\right)\end{array}$ & $\begin{array}{l}\text { elevation } \\
(\mathrm{m})\end{array}$ & $\begin{array}{c}\text { dip direction } \\
\left({ }^{\circ}\right)\end{array}$ & $\begin{array}{l}\text { dip } \\
\left({ }^{\circ}\right)\end{array}$ & $\begin{array}{l}\text { elevation } \\
(\mathrm{m})\end{array}$ \\
\hline 62 & 22 & 0,00 & 66 & 38 & 47.7 & 69 & 25 & 0 & 92 & 40 & 0 \\
\hline 73 & 29 & 0.14 & 65 & 33 & 47.71 & 90 & 32 & 1.91 & 83 & 37 & 0.13 \\
\hline 66 & 31 & 0.36 & 64 & 37 & 47.72 & 75 & 37 & 2.26 & 76 & 29 & 0.26 \\
\hline 69 & 19 & 1.43 & 60 & 37 & 47.73 & 91 & 43 & 3.62 & 69 & 29 & 0.39 \\
\hline 82 & 35 & 3.15 & 55 & 33 & 47.74 & 92 & 35 & 4.44 & 80 & 37 & 0.52 \\
\hline 89 & 25 & 3.67 & 50 & 32 & 47.78 & 45 & 36 & 5.47 & 72 & 30 & 0.65 \\
\hline 80 & 32 & 4.5 & 53 & 32 & 47.83 & 22 & 15 & 5.89 & 78 & 37 & 0.77 \\
\hline 91 & 31 & 4.65 & 3 & 19 & 47.89 & 89 & 26 & 6.69 & 61 & 28 & 1.08 \\
\hline 70 & 29 & 4.79 & 42 & 18 & 47.9 & 356 & 33 & 7.32 & 65 & 39 & 1.39 \\
\hline 78 & 26 & 4.99 & 10 & 20 & 47.92 & 35 & 32 & 7.6 & 66 & 48 & 1.86 \\
\hline 80 & 30 & 5.36 & 49 & 20 & 47.97 & 52 & 30 & 7.95 & 66 & 29 & 2.17 \\
\hline 80 & 42 & 5.51 & 60 & 27 & 49.62 & 72 & 41 & 8.07 & 88 & 28 & 5.42 \\
\hline 87 & 26 & 6.54 & 53 & 34 & 49.69 & 76 & 22 & 8.19 & 60 & 30 & 5.89 \\
\hline 74 & 35 & 6.6 & 58 & 40 & 50.2 & 42 & 41 & 8.34 & 59 & 27 & 6.81 \\
\hline 68 & 30 & 6.68 & 55 & 26 & 50.56 & 47 & 28 & 8.42 & 68 & 39 & 7.74 \\
\hline 76 & 31 & 6.75 & 57 & 33 & 50.89 & 44 & 35 & 8.58 & 62 & 25 & 11.31 \\
\hline 62 & 24 & 6.81 & 55 & 23 & 58.75 & 83 & 18 & 9.11 & 88 & 33 & 11.39 \\
\hline 73 & 32 & 7.24 & 68 & 22 & 59.18 & 44 & 21 & 9.85 & 90 & 22 & 11.47 \\
\hline 75 & 20 & 7.36 & 62 & 22 & 65.77 & 43 & 39 & 10.41 & 73 & 41 & 11.56 \\
\hline 65 & 24 & 7.58 & 65 & 16 & 70.07 & 123 & 29 & 10.89 & 72 & 32 & 11.64 \\
\hline 88 & 43 & 9.08 & 53 & 13 & 70.36 & 82 & 29 & 11.44 & 87 & 53 & 11.73 \\
\hline 77 & 33 & 9.53 & 51 & 15 & 70.79 & 98 & 28 & 11.68 & 98 & 29 & 11.81 \\
\hline 66 & 28 & 9.68 & 72 & 19 & 70.93 & 98 & 24 & 11.73 & 90 & 42 & 11.89 \\
\hline 69 & 25 & 11.11 & 85 & 28 & 71.8 & 80 & 35 & 11.78 & 60 & 28 & 11.98 \\
\hline 75 & 36 & 11.97 & 330 & 2 & 73.8 & 75 & 35 & 12.79 & 68 & 11 & 12.06 \\
\hline 73 & 38 & 13.69 & & & & 83 & 30 & 13.19 & 64 & 25 & 12.15 \\
\hline 78 & 38 & 13.75 & & & & 65 & 34 & 13.72 & 271 & 9 & 12.23 \\
\hline 94 & 30 & 16.04 & & & & 65 & 35 & 14.63 & 86 & 47 & 12.39 \\
\hline 65 & 22 & 16.33 & & & & 94 & 18 & 17.43 & 79 & 49 & 12.7 \\
\hline 71 & 22 & 17.76 & & & & 86 & 13 & 17.95 & 86 & 47 & 13.16 \\
\hline 66 & 30 & 18.05 & & & & 75 & 20 & 18.68 & 103 & 73 & 13.32 \\
\hline 68 & 30 & 18.34 & & & & 61 & 28 & 19.98 & 89 & 74 & 13.63 \\
\hline 38 & 12 & 21.2 & & & & 58 & 17 & 25,00 & 87 & 58 & 13.94 \\
\hline 72 & 40 & 21.92 & & & & 71 & 17 & 27.95 & 90 & 60 & 14.25 \\
\hline 82 & 24 & 22.5 & & & & 55 & 34 & 30.04 & 51 & 14 & 14.56 \\
\hline 82 & 16 & 22.67 & & & & 75 & 25 & 31.97 & 74 & 29 & 14.87 \\
\hline 95 & 40 & 22.75 & & & & 100 & 15 & 32.05 & 64 & 50 & 15.95 \\
\hline 43 & 11 & 22.9 & & & & 65 & 26 & 32.13 & 61 & 26 & 19.36 \\
\hline 77 & 26 & 22.98 & & & & 51 & 24 & 32.21 & 41 & 47 & 19.98 \\
\hline 64 & 10 & 23.03 & & & & 52 & 24 & 32.33 & 55 & 29 & 20.75 \\
\hline 63 & 19 & 24.17 & & & & 56 & 23 & 32.41 & 89 & 15 & 21.37 \\
\hline 77 & 28 & 24.19 & & & & 74 & 27 & 32.49 & 63 & 44 & 21.84 \\
\hline 74 & 23 & 24.2 & & & & 69 & 27 & 32.57 & 49 & 12 & 22.46 \\
\hline 68 & 8 & 24.24 & & & & 85 & 25 & 32.65 & 65 & 44 & 23.08 \\
\hline 69 & 19 & 24.27 & & & & 65 & 23 & 32.73 & 55 & 39 & 23.39 \\
\hline 35 & 12 & 24.35 & & & & 81 & 25 & 32.88 & 52 & 24 & 23.54 \\
\hline 45 & 26 & 24.41 & & & & 89 & 30 & 32.96 & 50 & 22 & 24,00 \\
\hline 64 & 23 & 24.53 & & & & 49 & 21 & 33.62 & 58 & 25 & 24.16 \\
\hline 63 & 21 & 24.58 & & & & 53 & 22 & 33.92 & 65 & 22 & 24.47 \\
\hline 75 & 29 & 24.61 & & & & 68 & 33 & 34.22 & 63 & 39 & 24.78 \\
\hline 65 & 22 & 25.47 & & & & 325 & 24 & 34.32 & 66 & 30 & 25.09 \\
\hline 70 & 25 & 25.56 & & & & 80 & 32 & 44.46 & 61 & 44 & 29.12 \\
\hline 73 & 36 & 25.59 & & & & 77 & 36 & 44.53 & 62 & 36 & 30.66 \\
\hline 82 & 34 & 25.6 & & & & 70 & 37 & 44.59 & 74 & 22 & 34.23 \\
\hline 77 & 29 & 25.61 & & & & 55 & 34 & 44.65 & 62 & 22 & 36.08 \\
\hline 57 & 20 & 25.79 & & & & 62 & 33 & 44.71 & 68 & 23 & 37.17 \\
\hline 50 & 28 & 25.79 & & & & 55 & 37 & 44.77 & 70 & 25 & 38.87 \\
\hline 79 & 29 & 25.82 & & & & 82 & 26 & 44.8 & 67 & 33 & 39.18 \\
\hline 69 & 14 & 25.83 & & & & 58 & 32 & 44.87 & 58 & 30 & 39.49 \\
\hline 85 & 24 & 26.12 & & & & 65 & 28 & 44.93 & 43 & 23 & 39.65 \\
\hline 68 & 34 & 26.15 & & & & 66 & 34 & 44.99 & 75 & 23 & 39.96 \\
\hline 82 & 26 & 26.17 & & & & 85 & 16 & 45.05 & 76 & 25 & 40.11 \\
\hline 76 & 27 & 26.4 & & & & 61 & 23 & 45.11 & 63 & 24 & 40.42 \\
\hline 64 & 30 & 27.39 & & & & 68 & 33 & 45.18 & 60 & 23 & 40.89 \\
\hline 62 & 29 & 31.21 & & & & 69 & 29 & 45.24 & 61 & 19 & 41.35 \\
\hline 70 & 24 & 31.78 & & & & 75 & 30 & 45.3 & 65 & 26 & 41.81 \\
\hline 75 & 22 & 34.82 & & & & 318 & 8 & 45.36 & 66 & 18 & 42.12 \\
\hline 65 & 20 & 34.91 & & & & 25 & 26 & 45.42 & 62 & 22 & 42.28 \\
\hline 80 & 30 & 34.96 & & & & 51 & 33 & 45.45 & 63 & 26 & 42.43 \\
\hline 70 & 25 & 35.05 & & & & 62 & 53 & 45.52 & 61 & 24 & 42.74 \\
\hline 77 & 25 & 35.22 & & & & 50 & 52 & 45.58 & 64 & 24 & 42.9 \\
\hline 39 & 27 & 35.59 & & & & 77 & 30 & 45.64 & 63 & 22 & 43.05 \\
\hline 32 & 24 & 35.6 & & & & 68 & 28 & 45.7 & 58 & 19 & 43.21 \\
\hline 50 & 24 & 35.61 & & & & & & & 73 & 26 & 43.36 \\
\hline 60 & 29 & 35.66 & & & & & & & 62 & 22 & 43.67 \\
\hline
\end{tabular}


\begin{tabular}{l|l|l}
64 & 30 & 47.67
\end{tabular} 


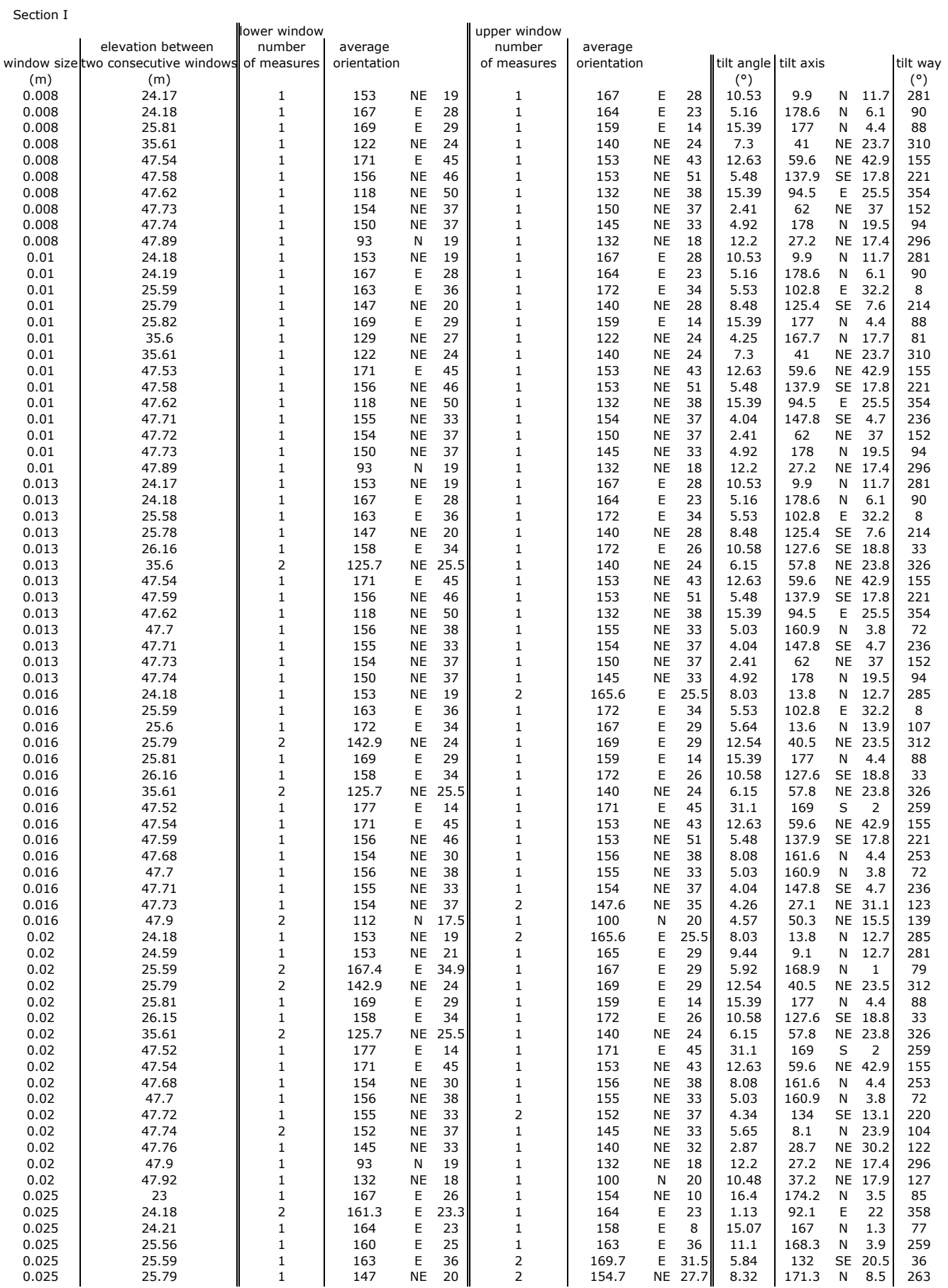




\begin{tabular}{|c|c|c|c|c|c|c|c|c|c|c|c|c|c|c|}
\hline 0.032 & 47.77 & 1 & 145 & $\mathrm{NE}$ & 33 & & 140 & $\mathrm{NE}$ & 32 & 2.87 & 28.7 & $\mathrm{NE}$ & 30.2 & 122 \\
\hline 0.032 & 47.8 & 1 & 140 & $\mathrm{NE}$ & 32 & & 143 & $\mathrm{NE}$ & 32 & 1.59 & 51.5 & $\mathrm{NE}$ & 32 & 321 \\
\hline 0.032 & 47.89 & 1 & 93 & $\mathrm{~N}$ & 19 & & 115.2 & $\mathrm{NE}$ & 18.3 & 7.08 & 19.8 & $\mathrm{~N}$ & 18.2 & 289 \\
\hline 0.04 & 6.79 & 1 & 166 & $\mathrm{E}$ & 31 & & 152 & $\mathrm{NE}$ & 24 & 9.49 & 18.5 & $\mathrm{~N}$ & 17.9 & 113 \\
\hline 0.04 & 13.72 & 1 & 163 & $E$ & 38 & & 168 & $\mathrm{E}$ & 38 & 3.08 & 75.5 & E & 38 & 345 \\
\hline 0.04 & 22.99 & 1 & 167 & $\mathrm{E}$ & 26 & & 154 & $\mathrm{NE}$ & 10 & 16.4 & 174.2 & $\mathrm{~N}$ & 3.5 & 85 \\
\hline 0.04 & 24.19 & 2 & 161.3 & E & 23.3 & & 164 & $\mathrm{E}$ & 23 & 1.13 & 92.1 & E & 22 & 358 \\
\hline 0.04 & 24.23 & 1 & 164 & $\mathrm{E}$ & 23 & & 158.7 & $\mathrm{E}$ & 13.5 & 9.63 & 170.8 & $\mathrm{~N}$ & 2.9 & 81 \\
\hline 0.04 & 24.38 & 1 & 125 & $\mathrm{NE}$ & 12 & & 135 & NE & 26 & 14.32 & 142.6 & NW & 3.7 & 233 \\
\hline 0.04 & 24.54 & 1 & 154 & $\mathrm{NE}$ & 23 & & 153 & $\mathrm{NE}$ & 21 & 2.03 & 163.4 & $\mathrm{~N}$ & 3.9 & 74 \\
\hline 0.04 & 24.58 & 1 & 153 & $\mathrm{NE}$ & 21 & & 165 & $\mathrm{E}$ & 29 & 9.44 & 9.1 & $\mathrm{~N}$ & 12.7 & 281 \\
\hline 0.04 & 25.58 & 1 & 160 & $\mathrm{E}$ & 25 & & 167.3 & $\mathrm{E}$ & 32.9 & 8.68 & 4.9 & $\mathrm{~N}$ & 11.1 & 277 \\
\hline 0.04 & 25.82 & 3 & 152.6 & $\mathrm{NE}$ & 25.1 & & 159 & E & 14 & 11.28 & 145.5 & SE & 3.3 & 55 \\
\hline 0.04 & 26.14 & 1 & 175 & $\mathrm{E}$ & 24 & & 164.1 & E & 29.8 & 7.59 & 132.5 & SE & 16.7 & 220 \\
\hline 0.04 & 34.93 & 1 & 155 & $\mathrm{NE}$ & 20 & & 170 & $\mathrm{E}$ & 30 & 11.77 & 12.6 & $\mathrm{~N}$ & 12.5 & 284 \\
\hline 0.04 & 36.37 & 1 & 124 & $\mathrm{NE}$ & 22 & & 148 & $\mathrm{NE}$ & 33 & 15.42 & 178.4 & $\mathrm{~N}$ & 18.2 & 270 \\
\hline 0.04 & 47.51 & 1 & 177 & $\mathrm{E}$ & 14 & & 162.2 & $\mathrm{E}$ & 43.6 & 30.28 & 157 & SE & 4.9 & 247 \\
\hline 0.04 & 47.55 & 2 & 162.2 & $\mathrm{E}$ & 43.6 & & 154.4 & $\mathrm{NE}$ & 48.5 & 7.37 & 119.7 & $\mathrm{SE}$ & 32.8 & 201 \\
\hline 0.04 & 47.59 & 2 & 154.4 & $\mathrm{NE}$ & 48.5 & & 124.2 & $\mathrm{NE}$ & 43.8 & 22.14 & 32.4 & $\mathrm{NE}$ & 43.8 & 132 \\
\hline 0.04 & 47.63 & 2 & 124.2 & $\mathrm{NE}$ & 43.8 & & 154 & $\mathrm{NE}$ & 30 & 22.25 & 92.2 & $\mathrm{E}$ & 27 & 353 \\
\hline 0.04 & 47.67 & 1 & 154 & $\mathrm{NE}$ & 30 & & 155.5 & $\mathrm{NE}$ & 35.5 & 5.56 & 162 & $\mathrm{~N}$ & 4.6 & 253 \\
\hline 0.04 & 47.71 & 2 & 155.5 & $\mathrm{NE}$ & 35.5 & & 149.8 & $\mathrm{NE}$ & 35.6 & 3.32 & 65.1 & $\mathrm{NE}$ & 35.5 & 155 \\
\hline 0.04 & 47.75 & 3 & 149.8 & $\mathrm{NE}$ & 35.6 & & 140 & $\mathrm{NE}$ & 32 & 6.54 & 16.5 & $N$ & 27.5 & 112 \\
\hline 0.04 & 47.79 & 1 & 140 & $\mathrm{NE}$ & 32 & & 143 & $\mathrm{NE}$ & 32 & 1.59 & 51.5 & $\mathrm{NE}$ & 32 & 321 \\
\hline 0.04 & 47.91 & 2 & 112 & $\mathrm{~N}$ & 17.5 & & 100 & $\mathrm{~N}$ & 20 & 4.57 & 50.3 & $\mathrm{NE}$ & 15.5 & 139 \\
\hline 0.04 & 47.95 & 1 & 100 & $\mathrm{~N}$ & 20 & & 139 & $\mathrm{NE}$ & 20 & 13.11 & 29.5 & NE & 18.9 & 298 \\
\hline 0.05 & 6.56 & 1 & 177 & $\mathrm{E}$ & 26 & & 164 & $\mathrm{E}$ & 35 & 11.11 & 138 & $\mathrm{SE}$ & 17.1 & 225 \\
\hline 0.05 & 6.71 & 1 & 158 & $\mathrm{E}$ & 30 & & 166 & $\mathrm{E}$ & 31 & 4.18 & 56.1 & $\mathrm{NE}$ & 29.5 & 328 \\
\hline 0.05 & 13.73 & 1 & 163 & $\mathrm{E}$ & 38 & & 168 & $\mathrm{E}$ & 38 & 3.08 & 75.5 & $\mathrm{E}$ & 38 & 345 \\
\hline 0.05 & 23 & 1 & 167 & E & 26 & & 154 & $\mathrm{NE}$ & 10 & 16.4 & 174.2 & $\mathrm{~N}$ & 3.5 & 85 \\
\hline 0.05 & 24.2 & 3 & 162.2 & E & 23.2 & & 158 & $\mathrm{E}$ & 8 & 15.26 & 164.2 & $\mathrm{~N}$ & 0.9 & 74 \\
\hline 0.05 & 24.25 & 1 & 158 & E & 8 & & 159 & $\mathrm{E}$ & 19 & 11 & 159.7 & $\mathrm{~N}$ & 0.2 & 250 \\
\hline 0.05 & 24.3 & 1 & 159 & E & 19 & & 125 & NE & 12 & 11.19 & 14.3 & $\mathrm{~N}$ & 11.2 & 106 \\
\hline 0.05 & 24.55 & 1 & 154 & $\mathrm{NE}$ & 23 & & 153 & $\mathrm{NE}$ & 21 & 2.03 & 163.4 & $\mathrm{~N}$ & 3.9 & 74 \\
\hline 0.05 & 24.6 & 1 & 153 & $\mathrm{NE}$ & 21 & & 165 & $\mathrm{E}$ & 29 & 9.44 & 9.1 & $\mathrm{~N}$ & 12.7 & 281 \\
\hline 0.05 & 25.5 & 1 & 155 & $\mathrm{NE}$ & 22 & & 160 & $\mathrm{E}$ & 25 & 3.6 & 8.9 & $\mathrm{~N}$ & 12.7 & 281 \\
\hline 0.05 & 25.55 & 1 & 160 & $\mathrm{E}$ & 25 & & 167.4 & E & 34.9 & 10.56 & 1.7 & $\mathrm{~N}$ & 9.8 & 274 \\
\hline 0.05 & 25.6 & 2 & 167.4 & $\bar{E}$ & 34.9 & & 167 & E & 29 & 5.92 & 168.9 & $\mathrm{~N}$ & 1 & 79 \\
\hline 0.05 & 25.8 & 2 & 142.9 & $\mathrm{NE}$ & 24 & & 165.7 & E & 21.4 & 9.07 & 81.4 & E & 21.3 & 350 \\
\hline 0.05 & 26.15 & 2 & 165.1 & $\mathrm{E}$ & 28.7 & & 172 & $E$ & 26 & 4.17 & 122.9 & SE & 20.2 & 29 \\
\hline 0.05 & 34.93 & 1 & 155 & $\mathrm{NE}$ & 20 & & 170 & E & 30 & 11.77 & 12.6 & $\mathrm{~N}$ & 12.5 & 284 \\
\hline 0.05 & 35.63 & 3 & 130.3 & $\mathrm{NE}$ & 24.8 & & 150 & $\mathrm{NE}$ & 29 & 9.8 & 22.7 & $\mathrm{NE}$ & 23.8 & 294 \\
\hline 0.05 & 36.38 & 1 & 124 & $\mathrm{NE}$ & 22 & & 148 & $\mathrm{NE}$ & 33 & 15.42 & 178.4 & $\mathrm{~N}$ & 18.2 & 270 \\
\hline 0.05 & 40.74 & 1 & 145 & $\mathrm{NE}$ & 12 & & 120 & $\mathrm{NE}$ & 19 & 9.53 & 89.4 & E & 10 & 179 \\
\hline 0.05 & 47.56 & 3 & 164.3 & E & 34 & & 154.4 & $\mathrm{NE}$ & 48.5 & 15.83 & 140.5 & $\mathrm{SE}$ & 15.2 & 226 \\
\hline 0.05 & 47.61 & 2 & 154.4 & $\mathrm{NE}$ & 48.5 & & 124.2 & $\mathrm{NE}$ & 43.8 & 22.14 & 32.4 & $\mathrm{NE}$ & 43.8 & 132 \\
\hline 0.05 & 47.66 & 2 & 124.2 & $\mathrm{NE}$ & 43.8 & & 155.1 & $\mathrm{NE}$ & 34 & 21.47 & 81.9 & $\mathrm{E}$ & 32.8 & 343 \\
\hline 0.05 & 47.71 & 2 & 155.1 & $\mathrm{NE}$ & 34 & & 151.1 & $\mathrm{NE}$ & 34.9 & 2.48 & 89.6 & $\mathrm{E}$ & 31.5 & 175 \\
\hline 0.05 & 47.76 & 4 & 151.1 & $\mathrm{NE}$ & 34.9 & & 140 & $\mathrm{NE}$ & 32 & 6.76 & 25.6 & $\mathrm{NE}$ & 29.6 & 121 \\
\hline 0.05 & 47.81 & 1 & 140 & $\mathrm{NE}$ & 32 & & 143 & $\mathrm{NE}$ & 32 & 1.59 & 51.5 & NE & 32 & 321 \\
\hline 0.05 & 47.86 & 1 & 143 & $\mathrm{NE}$ & 32 & & 112 & $\mathrm{~N}$ & 17.5 & 19.03 & 167.6 & $\mathrm{~N}$ & 14.6 & 81 \\
\hline 0.05 & 47.91 & 2 & 112 & $\mathrm{~N}$ & 17.5 & & 100 & $\mathrm{~N}$ & 20 & 4.57 & 50.3 & $\mathrm{NE}$ & 15.5 & 139 \\
\hline 0.05 & 47.96 & 1 & 100 & $\mathrm{~N}$ & 20 & & 139 & $\mathrm{NE}$ & 20 & 13.11 & 29.5 & $\mathrm{NE}$ & 18.9 & 298 \\
\hline 0.063 & 6.56 & 1 & 177 & E & 26 & & 164 & E & 35 & 11.11 & 138 & $\mathrm{SE}$ & 17.1 & 225 \\
\hline 0.063 & 6.62 & 1 & 164 & E & 35 & & 158 & E & 30 & 5.94 & 9.6 & $\mathrm{~N}$ & 16.8 & 104 \\
\hline 0.063 & 6.81 & 1 & 166 & E & 31 & & 152 & $\mathrm{NE}$ & 24 & 9.49 & 18.5 & $\mathrm{~N}$ & 17.9 & 113 \\
\hline 0.063 & 22.71 & 1 & 172 & $\mathrm{E}$ & 16 & & 5 & $\mathrm{~s}$ & 40 & 24.63 & 11.6 & $\mathrm{~N}$ & 5.5 & 282 \\
\hline 0.063 & 24.22 & 3 & 162.2 & $\mathrm{E}$ & 23.2 & & 158.7 & E & 13.5 & 9.78 & 166.6 & $\mathrm{~N}$ & 1.9 & 77 \\
\hline 0.063 & 24.54 & 1 & 154 & $\mathrm{NE}$ & 23 & & 153 & $\mathrm{NE}$ & 21 & 2.03 & 163.4 & $\mathrm{~N}$ & 3.9 & 74 \\
\hline 0.063 & 24.6 & 1 & 153 & $\mathrm{NE}$ & 21 & & 165 & E & 29 & 9.44 & 9.1 & $\mathrm{~N}$ & 12.7 & 281 \\
\hline 0.063 & 25.8 & 2 & 142.9 & $\mathrm{NE}$ & 24 & & 165.7 & E & 21.4 & 9.07 & 81.4 & E & 21.3 & 350 \\
\hline 0.063 & 26.12 & 1 & 175 & $\mathrm{E}$ & 24 & & 164.1 & $\mathrm{E}$ & 29.8 & 7.59 & 132.5 & SE & 16.7 & 220 \\
\hline 0.063 & 34.95 & 1 & 155 & $\mathrm{NE}$ & 20 & & 170 & E & 30 & 11.77 & 12.6 & $\mathrm{~N}$ & 12.5 & 284 \\
\hline 0.063 & 35.01 & 1 & 170 & $E$ & 30 & & 160 & E & 25 & 6.79 & 24.4 & $\mathrm{NE}$ & 18.1 & 118 \\
\hline 0.063 & 35.64 & 3 & 130.3 & $\mathrm{NE}$ & 24.8 & & 150 & $\mathrm{NE}$ & 29 & 9.8 & 22.7 & $\mathrm{NE}$ & 23.8 & 294 \\
\hline 0.063 & 39.74 & 1 & 106 & $\mathrm{~N}$ & 16 & & 107 & $\mathrm{~N}$ & 13 & 3.01 & 101.9 & E & 1.2 & 12 \\
\hline 0.063 & 41.07 & 1 & 176 & E & 11 & & 20 & S & 25 & 15.57 & 35.3 & $\mathrm{NE}$ & 7 & 306 \\
\hline 0.063 & 43.91 & 1 & 160 & $\mathrm{E}$ & 18 & & 157 & $\mathrm{NE}$ & 29 & 11.06 & 152.8 & $\mathrm{SE}$ & 2.3 & 242 \\
\hline 0.063 & 47.57 & 3 & 164.3 & $\mathrm{E}$ & 34 & & 140.2 & $\mathrm{NE}$ & 45.1 & 18.75 & 104.9 & $\mathrm{E}$ & 30.1 & 190 \\
\hline 0.063 & 47.63 & 4 & 140.2 & $\mathrm{NE}$ & 45.1 & & 155.1 & $\mathrm{NE}$ & 34 & 14.54 & 114.1 & SE & 23.9 & 15 \\
\hline 0.063 & 47.69 & 2 & 155.1 & $\mathrm{NE}$ & 34 & & 151.1 & $\mathrm{NE}$ & 34.9 & 2.48 & 89.6 & $\mathrm{E}$ & 31.5 & 175 \\
\hline 0.063 & 47.76 & 4 & 151.1 & $\mathrm{NE}$ & 34.9 & & 140 & $\mathrm{NE}$ & 32 & 6.76 & 25.6 & $\mathrm{NE}$ & 29.6 & 121 \\
\hline 0.063 & 47.82 & 1 & 140 & $\mathrm{NE}$ & 32 & & 143 & $\mathrm{NE}$ & 32 & 1.59 & 51.5 & $\mathrm{NE}$ & 32 & 321 \\
\hline 0.063 & 47.88 & 1 & 143 & $\mathrm{NE}$ & 32 & & 107.6 & $\mathrm{~N}$ & 18.3 & 19.84 & 171.2 & $\mathrm{~N}$ & 16.5 & 85 \\
\hline 0.063 & 47.95 & 3 & 107.6 & $\mathrm{~N}$ & 18.3 & & 139 & $\mathrm{NE}$ & 20 & 10.32 & 23.4 & $\mathrm{NE}$ & 18.2 & 293 \\
\hline 0.063 & 49.65 & 1 & 150 & $\mathrm{NE}$ & 27 & & 143 & $\mathrm{NE}$ & 34 & 7.84 & 122.8 & $\mathrm{SE}$ & 13.1 & 210 \\
\hline 0.079 & 4.58 & 1 & 170 & $\mathrm{E}$ & 32 & & 1 & $\mathrm{~s}$ & 31 & 5.83 & 97 & E & 30.9 & 5 \\
\hline 0.079 & 6.56 & 1 & 177 & E & 26 & & 164 & E & 35 & 11.11 & 138 & SE & 17.1 & 225 \\
\hline 0.079 & 6.64 & 1 & 164 & E & 35 & & 158 & E & 30 & 5.94 & 9.6 & $\mathrm{~N}$ & 16.8 & 104 \\
\hline
\end{tabular}




\begin{tabular}{|c|c|c|c|c|c|c|c|c|c|c|c|c|c|c|}
\hline 0.079 & 47.87 & 1 & 143 & $\mathrm{NE}$ & 32 & 3 & 107.6 & $\mathrm{~N}$ & 18.3 & 19.84 & 171.2 & $\mathrm{~N}$ & 16.5 & 85 \\
\hline 0.079 & 47.95 & 3 & 107.6 & $\mathrm{~N}$ & 18.3 & 1 & 139 & $\mathrm{NE}$ & 20 & 10.32 & 23.4 & NE & 18.2 & 293 \\
\hline 0.079 & 49.61 & 1 & 150 & $\mathrm{NE}$ & 27 & 1 & 143 & $\mathrm{NE}$ & 34 & 7.84 & 122.8 & SE & 13.1 & 210 \\
\hline 0.1 & 0.06 & 1 & 152 & $\mathrm{NE}$ & 22 & 1 & 163 & $E$ & 29 & 8.42 & 9.1 & $\mathrm{~N}$ & 13.7 & 281 \\
\hline 0.1 & 4.56 & 1 & 170 & $\mathrm{E}$ & 32 & 1 & 1 & $\mathrm{~s}$ & 31 & 5.83 & 97 & $E$ & 30.9 & 5 \\
\hline 0.1 & 5.46 & 1 & 170 & $\mathrm{E}$ & 30 & 1 & 170 & $\mathrm{E}$ & 42 & 12 & 170 & $\mathrm{~N}$ & 0 & 260 \\
\hline 0.1 & 6.56 & 1 & 177 & $\mathrm{E}$ & 26 & 1 & 164 & E & 35 & 11.11 & 138 & SE & 17.1 & 225 \\
\hline 0.1 & 6.66 & 1 & 164 & $\mathrm{E}$ & 35 & 2 & 162.1 & $\mathrm{E}$ & 30.4 & 4.68 & 174 & $\mathrm{~N}$ & 6.9 & 86 \\
\hline 0.1 & 6.76 & 2 & 162.1 & $\mathrm{E}$ & 30.4 & 1 & 152 & $\mathrm{NE}$ & 24 & 7.89 & 9.6 & $\mathrm{~N}$ & 15.2 & 103 \\
\hline 0.1 & 7.26 & 1 & 163 & $\mathrm{E}$ & 32 & 1 & 165 & $\mathrm{E}$ & 20 & 12.03 & 160.2 & $\mathrm{~S}$ & 1.7 & 70 \\
\hline 0.1 & 22.96 & 1 & 133 & $\mathrm{NE}$ & 11 & 2 & 163.3 & $\mathrm{E}$ & 17.9 & 10.03 & 15.6 & $\mathrm{~N}$ & 9.8 & 286 \\
\hline 0.1 & 24.26 & 4 & 161.7 & $\mathrm{E}$ & 19.5 & 2 & 145.9 & $\mathrm{NE}$ & 14.9 & 6.51 & 18.4 & $\mathrm{~N}$ & 11.9 & 110 \\
\hline 0.1 & 24.36 & 2 & 145.9 & $\mathrm{NE}$ & 14.9 & 1 & 135 & $\mathrm{NE}$ & 26 & 11.7 & 122.5 & SE & 6 & 212 \\
\hline 0.1 & 24.46 & 1 & 135 & $\mathrm{NE}$ & 26 & 1 & 154 & $\mathrm{NE}$ & 23 & 8.39 & 77 & $\mathrm{E}$ & 22.5 & 344 \\
\hline 0.1 & 24.56 & 1 & 154 & $\mathrm{NE}$ & 23 & 2 & 159.9 & $\mathrm{E}$ & 24.9 & 3.04 & 26.3 & NE & 18.6 & 298 \\
\hline 0.1 & 25.56 & 2 & 157.7 & $\mathrm{E}$ & 23.5 & 3 & 167.3 & $\mathrm{E}$ & 32.9 & 10.47 & 5.6 & $\mathrm{~N}$ & 11.5 & 278 \\
\hline 0.1 & 26.16 & 2 & 165.1 & $\mathrm{E}$ & 28.7 & 1 & 172 & $\mathrm{E}$ & 26 & 4.17 & 122.9 & SE & 20.2 & 29 \\
\hline 0.1 & 34.86 & 1 & 165 & $E$ & 22 & 1 & 155 & $\mathrm{NE}$ & 20 & 4.1 & 39.2 & NE & 18.1 & 131 \\
\hline 0.1 & 34.96 & 1 & 155 & $\mathrm{NE}$ & 20 & 2 & 165.4 & $\mathrm{E}$ & 27.4 & 8.49 & 7.7 & $\mathrm{~N}$ & 11.1 & 279 \\
\hline 0.1 & 35.66 & 3 & 130.3 & $\mathrm{NE}$ & 24.8 & 1 & 150 & $\mathrm{NE}$ & 29 & 9.8 & 22.7 & $\mathrm{NE}$ & 23.8 & 294 \\
\hline 0.1 & 35.76 & 1 & 150 & $\mathrm{NE}$ & 29 & 1 & 140 & $\mathrm{NE}$ & 30 & 5.02 & 68.1 & $\mathrm{E}$ & 28.8 & 157 \\
\hline 0.1 & 36.36 & 1 & 124 & $\mathrm{NE}$ & 22 & 1 & 148 & $\mathrm{NE}$ & 33 & 15.42 & 178.4 & $\mathrm{~N}$ & 18.2 & 270 \\
\hline 0.1 & 36.46 & 1 & 148 & $\mathrm{NE}$ & 33 & 1 & 143 & $\mathrm{NE}$ & 32 & 2.87 & 31.7 & NE & 30.2 & 125 \\
\hline 0.1 & 39.75 & 1 & 106 & $\mathrm{~N}$ & 16 & 1 & 107 & $\mathrm{~N}$ & 13 & 3.01 & 101.9 & $\mathrm{E}$ & 1.2 & 12 \\
\hline 0.1 & 40.95 & 1 & 137 & $\mathrm{NE}$ & 15 & 1 & 176 & E & 11 & 9.4 & 90.7 & $\mathrm{E}$ & 11 & 360 \\
\hline 0.1 & 41.05 & 1 & 176 & $\mathrm{E}$ & 11 & 1 & 20 & $\mathrm{~s}$ & 25 & 15.57 & 35.3 & NE & 7 & 306 \\
\hline 0.1 & 47.55 & 3 & 164.3 & $\mathrm{E}$ & 34 & 4 & 140.2 & $\mathrm{NE}$ & 45.1 & 18.75 & 104.9 & $\mathrm{E}$ & 30.1 & 190 \\
\hline 0.1 & 47.65 & 4 & 140.2 & $\mathrm{NE}$ & 45.1 & 6 & 152.4 & $\mathrm{NE}$ & 34.6 & 13.03 & 116.5 & SE & 22 & 18 \\
\hline 0.1 & 47.75 & 6 & 152.4 & $\mathrm{NE}$ & 34.6 & 2 & 141.5 & $\mathrm{NE}$ & 32 & 6.51 & 29.3 & $\mathrm{NE}$ & 30 & 124 \\
\hline 0.1 & 47.85 & 2 & 141.5 & $\mathrm{NE}$ & 32 & 3 & 107.6 & $\mathrm{~N}$ & 18.3 & 19.41 & 169.2 & $\mathrm{~N}$ & 16.2 & 83 \\
\hline 0.1 & 47.95 & 3 & 107.6 & $\mathrm{~N}$ & 18.3 & 1 & 139 & $\mathrm{NE}$ & 20 & 10.32 & 23.4 & NE & 18.2 & 293 \\
\hline 0.1 & 49.65 & 1 & 150 & $\mathrm{NE}$ & 27 & 1 & 143 & $\mathrm{NE}$ & 34 & 7.84 & 122.8 & $\mathrm{SE}$ & 13.1 & 210 \\
\hline 0.1 & 70.85 & 1 & 141 & $\mathrm{NE}$ & 15 & 1 & 162 & $E$ & 19 & 7.27 & 27.6 & NE & 13.8 & 298 \\
\hline 0.126 & 0.08 & 1 & 152 & $\mathrm{NE}$ & 22 & 1 & 163 & $\mathrm{E}$ & 29 & 8.42 & 9.1 & $\mathrm{~N}$ & 13.7 & 281 \\
\hline 0.126 & 4.61 & 1 & 170 & $\mathrm{E}$ & 32 & 1 & 1 & $\mathrm{~s}$ & 31 & 5.83 & 97 & $\mathrm{E}$ & 30.9 & 5 \\
\hline 0.126 & 4.74 & 1 & 1 & $\mathrm{~s}$ & 31 & 1 & 160 & E & 29 & 10.64 & 68.2 & $\mathrm{E}$ & 29 & 161 \\
\hline 0.126 & 4.87 & 1 & 160 & $\mathrm{E}$ & 29 & 1 & 168 & E & 26 & 4.75 & 116.4 & SE & 20.9 & 23 \\
\hline 0.126 & 6.63 & 2 & 169.6 & $\mathrm{E}$ & 30.3 & 2 & 162.1 & $\mathrm{E}$ & 30.4 & 3.83 & 77.5 & $\mathrm{E}$ & 30.3 & 168 \\
\hline 0.126 & 6.75 & 2 & 162.1 & $\mathrm{E}$ & 30.4 & 1 & 152 & $\mathrm{NE}$ & 24 & 7.89 & 9.6 & $\mathrm{~N}$ & 15.2 & 103 \\
\hline 0.126 & 7.26 & 1 & 163 & $\mathrm{E}$ & 32 & 1 & 165 & $\mathrm{E}$ & 20 & 12.03 & 160.2 & $\mathrm{~s}$ & 1.7 & 70 \\
\hline 0.126 & 9.65 & 1 & 167 & $\mathrm{E}$ & 33 & & 156 & $\mathrm{NE}$ & 28 & 7.48 & 25.5 & $\mathrm{NE}$ & 22 & 121 \\
\hline 0.126 & 22.74 & 1 & 172 & E & 16 & 1 & 5 & $\mathrm{~s}$ & 40 & 24.63 & 11.6 & $N$ & 5.5 & 282 \\
\hline 0.126 & 22.87 & 1 & 5 & $\mathrm{~s}$ & 40 & 2 & 156.9 & $\mathrm{NE}$ & 17.9 & 25.45 & 20.3 & $\mathrm{~N}$ & 12.5 & 115 \\
\hline 0.126 & 22.99 & 2 & 156.9 & $\mathrm{NE}$ & 17.9 & 1 & 154 & $\mathrm{NE}$ & 10 & 7.89 & 160.3 & $\mathrm{~N}$ & 1.1 & 71 \\
\hline 0.126 & 24.25 & 4 & 161.7 & $\mathrm{E}$ & 19.5 & 2 & 145.9 & $\mathrm{NE}$ & 14.9 & 6.51 & 18.4 & $\mathrm{~N}$ & 11.9 & 110 \\
\hline 0.126 & 24.38 & 2 & 145.9 & $\mathrm{NE}$ & 14.9 & 1 & 135 & $\mathrm{NE}$ & 26 & 11.7 & 122.5 & SE & 6 & 212 \\
\hline 0.126 & 24.5 & 1 & 135 & $\mathrm{NE}$ & 26 & 3 & 158 & $\mathrm{E}$ & 24.2 & 9.87 & 67.7 & $\mathrm{E}$ & 24.2 & 336 \\
\hline 0.126 & 25.51 & 1 & 155 & $\mathrm{NE}$ & 22 & 4 & 165.8 & $\mathrm{E}$ & 30.9 & 10.1 & 6.3 & $\mathrm{~N}$ & 11.8 & 278 \\
\hline 0.126 & 26.14 & 1 & 175 & $\mathrm{E}$ & 24 & 2 & 164.1 & $\mathrm{E}$ & 29.8 & 7.59 & 132.5 & SE & 16.7 & 220 \\
\hline 0.126 & 34.83 & 1 & 165 & $\mathrm{E}$ & 22 & 1 & 155 & $\mathrm{NE}$ & 20 & 4.1 & 39.2 & $\mathrm{NE}$ & 18.1 & 131 \\
\hline 0.126 & 34.95 & 1 & 155 & $\mathrm{NE}$ & 20 & 2 & 165.4 & $E$ & 27.4 & 8.49 & 7.7 & $\mathrm{~N}$ & 11.1 & 279 \\
\hline 0.126 & 35.71 & 4 & 135.7 & $\mathrm{NE}$ & 25.6 & 1 & 140 & $\mathrm{NE}$ & 30 & 4.83 & 159.8 & $\mathrm{~N}$ & 11 & 252 \\
\hline 0.126 & 36.46 & 2 & 138.3 & $\mathrm{NE}$ & 27 & 1 & 143 & $\mathrm{NE}$ & 32 & 5.51 & 162.8 & $\mathrm{~N}$ & 12 & 255 \\
\hline 0.126 & 38.23 & 1 & 131 & $\mathrm{NE}$ & 28 & 1 & 106 & $\mathrm{~N}$ & 10 & 19.37 & 142.3 & NW & 6 & 54 \\
\hline 0.126 & 39.74 & 1 & 106 & $\mathrm{~N}$ & 16 & 1 & 107 & $\mathrm{~N}$ & 13 & 3.01 & 101.9 & E & 1.2 & 12 \\
\hline 0.126 & 40.74 & 1 & 145 & $\mathrm{NE}$ & 12 & 1 & 120 & $\mathrm{NE}$ & 19 & 9.53 & 89.4 & E & 10 & 179 \\
\hline 0.126 & 40.87 & 1 & 120 & $\mathrm{NE}$ & 19 & 1 & 137 & $\mathrm{NE}$ & 15 & 6.34 & 78.4 & $\mathrm{E}$ & 12.9 & 347 \\
\hline 0.126 & 41 & 1 & 137 & $\mathrm{NE}$ & 15 & 2 & 12.6 & $\mathrm{E}$ & 17.7 & 15.27 & 65.5 & $\mathrm{NE}$ & 14.3 & 335 \\
\hline 0.126 & 43.89 & 1 & 160 & $\mathrm{E}$ & 18 & 1 & 157 & $\mathrm{NE}$ & 29 & 11.06 & 152.8 & $\mathrm{SE}$ & 2.3 & 242 \\
\hline 0.126 & 47.54 & 3 & 164.3 & $\mathrm{E}$ & 34 & 4 & 140.2 & $\mathrm{NE}$ & 45.1 & 18.75 & 104.9 & $\mathrm{E}$ & 30.1 & 190 \\
\hline 0.126 & 47.67 & 4 & 140.2 & $\mathrm{NE}$ & 45.1 & 7 & 150.7 & $\mathrm{NE}$ & 34.2 & 12.8 & 120.1 & SE & 19.1 & 23 \\
\hline 0.126 & 47.79 & 7 & 150.7 & $\mathrm{NE}$ & 34.2 & 4 & 120.1 & $\mathrm{NE}$ & 20.8 & 19.09 & 179.5 & $\mathrm{~N}$ & 18.1 & 94 \\
\hline 0.126 & 47.92 & 4 & 120.1 & $\mathrm{NE}$ & 20.8 & 1 & 139 & $\mathrm{NE}$ & 20 & 6.61 & 46.5 & $\mathrm{NE}$ & 20 & 316 \\
\hline 0.126 & 70.83 & 1 & 141 & $\mathrm{NE}$ & 15 & 1 & 162 & $\mathrm{E}$ & 19 & 7.27 & 27.6 & $\mathrm{NE}$ & 13.8 & 298 \\
\hline 0.158 & 0.14 & 1 & 152 & $\mathrm{NE}$ & 22 & 1 & 163 & $\mathrm{E}$ & 29 & 8.42 & 9.1 & $\mathrm{~N}$ & 13.7 & 281 \\
\hline 0.158 & 0.29 & 1 & 163 & $\mathrm{E}$ & 29 & 1 & 156 & $\mathrm{NE}$ & 31 & 4.03 & 102.9 & E & 25.7 & 190 \\
\hline 0.158 & 4.57 & 1 & 170 & $\mathrm{E}$ & 32 & 1 & 1 & $\mathrm{~s}$ & 31 & 5.83 & 97 & $\mathrm{E}$ & 30.9 & 5 \\
\hline 0.158 & 4.73 & 1 & 1 & $\mathrm{~s}$ & 31 & 1 & 160 & $\mathrm{E}$ & 29 & 10.64 & 68.2 & $\mathrm{E}$ & 29 & 161 \\
\hline 0.158 & 4.89 & 1 & 160 & $\mathrm{E}$ & 29 & 1 & 168 & $\mathrm{E}$ & 26 & 4.75 & 116.4 & $\mathrm{SE}$ & 20.9 & 23 \\
\hline 0.158 & 5.37 & 1 & 170 & $\mathrm{E}$ & 30 & 1 & 170 & $\mathrm{E}$ & 42 & 12 & 170 & $\mathrm{~N}$ & 0 & 260 \\
\hline 0.158 & 6.63 & 2 & 169.6 & $\mathrm{E}$ & 30.3 & 2 & 162.1 & $E$ & 30.4 & 3.83 & 77.5 & E & 30.3 & 168 \\
\hline 0.158 & 6.79 & 2 & 162.1 & E & 30.4 & 1 & 152 & $\mathrm{NE}$ & 24 & 7.89 & 9.6 & $\mathrm{~N}$ & 15.2 & 103 \\
\hline 0.158 & 7.27 & 1 & 163 & $\mathrm{E}$ & 32 & 1 & 165 & $\mathrm{E}$ & 20 & 12.03 & 160.2 & $\mathrm{~s}$ & 1.7 & 70 \\
\hline 0.158 & 7.43 & 1 & 165 & $E$ & 20 & 1 & 155 & $\mathrm{NE}$ & 24 & 5.47 & 118.9 & SE & 14.7 & 207 \\
\hline 0.158 & 9.64 & 1 & 167 & $\mathrm{E}$ & 33 & 1 & 156 & $\mathrm{NE}$ & 28 & 7.48 & 25.5 & $\mathrm{NE}$ & 22 & 121 \\
\hline 0.158 & 18.2 & 1 & 156 & $\mathrm{NE}$ & 30 & 1 & 158 & E & 30 & 1 & 67 & $\mathrm{NE}$ & 30 & 337 \\
\hline 0.158 & 22.64 & 1 & 172 & E & 24 & 2 & 1.1 & E & 27.9 & 5.55 & 39.4 & $\mathrm{NE}$ & 18.1 & 312 \\
\hline 0.158 & 22.8 & 2 & 1.1 & $\mathrm{E}$ & 27.9 & 1 & 133 & $\mathrm{NE}$ & 11 & 21.98 & 21 & $\mathrm{~N}$ & 10.2 & 113 \\
\hline 0.158 & 22.96 & 1 & 133 & $\mathrm{NE}$ & 11 & 2 & 163.3 & $\mathrm{E}$ & 17.9 & 10.03 & 15.6 & $\mathrm{~N}$ & 9.8 & 286 \\
\hline
\end{tabular}




\begin{tabular}{|c|c|c|c|c|c|c|c|c|c|c|c|c|c|c|}
\hline 0.2 & 4.58 & 1 & 170 & $E$ & 32 & 2 & 170.8 & $\mathrm{E}$ & 29.6 & 2.45 & 161.9 & S & 5 & 71 \\
\hline 0.2 & 4.78 & 2 & 170.8 & $\mathrm{E}$ & 29.6 & 1 & 168 & $\mathrm{E}$ & 26 & 3.82 & 7.4 & $\mathrm{~N}$ & 9.2 & 100 \\
\hline 0.2 & 5.38 & 1 & 170 & $\mathrm{E}$ & 30 & 1 & 170 & $\mathrm{E}$ & 42 & 12 & 170 & $\mathrm{~N}$ & 0 & 260 \\
\hline 0.2 & 6.58 & 1 & 177 & $E$ & 26 & 3 & 162.8 & $E$ & 32 & 9.08 & 124.3 & SE & 21.2 & 212 \\
\hline 0.2 & 6.78 & 3 & 162.8 & $E$ & 32 & 1 & 152 & $\mathrm{NE}$ & 24 & 9.39 & 6.8 & $\mathrm{~N}$ & 14.3 & 100 \\
\hline 0.2 & 9.57 & 1 & 167 & $\mathrm{E}$ & 33 & 1 & 156 & $\mathrm{NE}$ & 28 & 7.48 & 25.5 & $\mathrm{NE}$ & 22 & 121 \\
\hline 0.2 & 16.15 & 1 & 4 & $\mathrm{~s}$ & 30 & 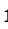 & 155 & $\mathrm{NE}$ & 22 & 14.81 & 45.2 & $\mathrm{NE}$ & 20.8 & 139 \\
\hline 0.2 & 17.95 & 1 & 161 & $\mathrm{E}$ & 22 & 1 & 156 & $\mathrm{NE}$ & 30 & 8.29 & 144.6 & SE & 6.5 & 233 \\
\hline 0.2 & 18.15 & 1 & 156 & $\mathrm{NE}$ & 30 & 1 & 158 & $\mathrm{E}$ & 30 & 1 & 67 & $\mathrm{NE}$ & 30 & 337 \\
\hline 0.2 & 22.54 & 1 & 172 & $\mathrm{E}$ & 24 & 1 & 172 & $\mathrm{E}$ & 16 & 8 & 172 & $\mathrm{~S}$ & 0 & 82 \\
\hline 0.2 & 22.74 & 1 & 172 & $\mathrm{E}$ & 16 & 2 & 173.8 & $\mathrm{E}$ & 23.9 & 7.94 & 177.1 & $\mathrm{~N}$ & 1.5 & 267 \\
\hline 0.2 & 22.94 & 2 & 173.8 & $\mathrm{E}$ & 23.9 & 2 & 163.3 & $E$ & 17.9 & 7.05 & 18.9 & $\mathrm{~N}$ & 10.7 & 111 \\
\hline 0.2 & 24.33 & 5 & 161.2 & $E$ & 19.4 & 3 & 140.2 & $\mathrm{NE}$ & 20 & 7.07 & 66 & $\mathrm{NE}$ & 19.3 & 156 \\
\hline 0.2 & 24.53 & 3 & 140.2 & $\mathrm{NE}$ & 20 & 2 & 159.9 & $E$ & 24.9 & 8.92 & 25.2 & $\mathrm{NE}$ & 18.3 & 297 \\
\hline 0.2 & 25.53 & 1 & 155 & $\mathrm{NE}$ & 22 & 8 & 165.8 & $\mathrm{E}$ & 30.9 & 10.1 & 6.3 & $\mathrm{~N}$ & 11.8 & 278 \\
\hline 0.2 & 25.73 & 4 & 165.8 & $\mathrm{E}$ & 30.9 & 8 & 153.6 & $\mathrm{NE}$ & 22.3 & 10.16 & 9.3 & $\mathrm{~N}$ & 13.4 & 103 \\
\hline 0.2 & 25.93 & 4 & 153.6 & $\mathrm{NE}$ & 22.3 & 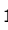 & 175 & $E$ & 24 & 8.52 & 62 & $\mathrm{NE}$ & 22.3 & 332 \\
\hline 0.2 & 26.13 & 1 & 175 & $E$ & 24 & 2 & 164.1 & $E$ & 29.8 & 7.59 & 132.5 & SE & 16.7 & 220 \\
\hline 0.2 & 26.33 & 2 & 164.1 & $\mathrm{E}$ & 29.8 & 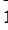 & 166 & $\mathrm{E}$ & 27 & 2.95 & 149.7 & $\mathrm{SE}$ & 8.1 & 57 \\
\hline 0.2 & 34.91 & 2 & 160.2 & $\mathrm{E}$ & 20.9 & 2 & 165.4 & $\mathrm{E}$ & 27.4 & 6.82 & 179.5 & $\mathrm{~N}$ & 7.2 & 271 \\
\hline 0.2 & 35.11 & 2 & 165.4 & $\mathrm{E}$ & 27.4 & . & 167 & $\mathrm{E}$ & 25 & 2.51 & 151.7 & $\mathrm{SE}$ & 7 & 60 \\
\hline 0.2 & 35.71 & 4 & 135.7 & $\mathrm{NE}$ & 25.6 & 1 & 140 & $\mathrm{NE}$ & 30 & 4.83 & 159.8 & $\mathrm{~N}$ & 11 & 252 \\
\hline 0.2 & 36.51 & 2 & 138.3 & $\mathrm{NE}$ & 27 & 1 & 143 & $\mathrm{NE}$ & 32 & 5.51 & 162.8 & $\mathrm{~N}$ & 12 & 255 \\
\hline 0.2 & 38.1 & 1 & 5 & $\mathrm{~S}$ & 35 & 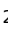 & 124.3 & $\mathrm{NE}$ & 18.7 & 30.01 & 33.9 & $\mathrm{NE}$ & 18.7 & 129 \\
\hline 0.2 & 39.7 & 1 & 106 & $\mathrm{~N}$ & 16 & . & 107 & $\mathrm{~N}$ & 13 & 3.01 & 101.9 & $\mathrm{E}$ & 1.2 & 12 \\
\hline 0.2 & 40.9 & 2 & 129.7 & $\mathrm{NE}$ & 15.2 & 2 & 153.4 & $\mathrm{NE}$ & 12.3 & 6.26 & 78.9 & $\mathrm{E}$ & 11.9 & 348 \\
\hline 0.2 & 41.1 & 2 & 153.4 & $\mathrm{NE}$ & 12.3 & 1 & 20 & $\mathrm{~s}$ & 25 & 18.67 & 46.6 & $\mathrm{NE}$ & 11.8 & 317 \\
\hline 0.2 & 43.09 & 1 & 175 & $E$ & 43 & 1 & 153 & $\mathrm{NE}$ & 17 & 27.84 & 5 & $\mathrm{~N}$ & 9.2 & 99 \\
\hline 0.2 & 43.89 & 1 & 160 & $E$ & 18 & 1 & 157 & $\mathrm{NE}$ & 29 & 11.06 & 152.8 & SE & 2.3 & 242 \\
\hline 0.2 & 47.68 & 8 & 149.6 & $\mathrm{NE}$ & 38.6 & 7 & 149.3 & $\mathrm{NE}$ & 34.4 & 4.22 & 151.8 & NW & 1.7 & 62 \\
\hline 0.2 & 47.88 & 7 & 149.3 & $\mathrm{NE}$ & 34.4 & 8 & 115.9 & $\mathrm{NE}$ & 18.2 & 21.42 & 173 & $\mathrm{~N}$ & 15.4 & 87 \\
\hline 0.2 & 49.67 & 1 & 150 & $\mathrm{NE}$ & 27 & 1 & 143 & $\mathrm{NE}$ & 34 & 7.84 & 122.8 & SE & 13.1 & 210 \\
\hline 0.2 & 70.23 & 1 & 155 & $\mathrm{NE}$ & 16 & 1 & 143 & $\mathrm{NE}$ & 13 & 4.23 & 13.2 & $\mathrm{~N}$ & 10.1 & 104 \\
\hline 0.2 & 70.82 & 1 & 141 & $\mathrm{NE}$ & 15 & 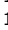 & 162 & $\mathrm{E}$ & 19 & 7.27 & 27.6 & $\mathrm{NE}$ & 13.8 & 298 \\
\hline 0.251 & 0.23 & 2 & 158.2 & $\mathrm{E}$ & 25.4 & 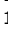 & 156 & $\mathrm{NE}$ & 31 & 5.7 & 147.8 & $\mathrm{SE}$ & 4.9 & 237 \\
\hline 0.251 & 4.5 & 1 & 170 & $\mathrm{E}$ & 32 & 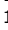 & 1 & $\mathrm{~s}$ & 31 & 5.83 & 97 & $\mathrm{E}$ & 30.9 & 5 \\
\hline 0.251 & 4.75 & 1 & 1 & $\mathrm{~s}$ & 31 & 2 & 163.8 & $\mathrm{E}$ & 27.4 & 9.08 & 56.7 & $\mathrm{NE}$ & 26.4 & 150 \\
\hline 0.251 & 6.76 & 4 & 165.8 & $E$ & 30.3 & 1 & 152 & $\mathrm{NE}$ & 24 & 8.91 & 20.6 & $\mathrm{~N}$ & 18.5 & 115 \\
\hline 0.251 & 7.01 & 1 & 152 & $\mathrm{NE}$ & 24 & 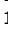 & 163 & $\mathrm{E}$ & 32 & 9.49 & 7.3 & $\mathrm{~N}$ & 14.4 & 280 \\
\hline 0.251 & 7.26 & 1 & 163 & $\mathrm{E}$ & 32 & 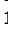 & 165 & $\mathrm{E}$ & 20 & 12.03 & 160.2 & $\mathrm{~s}$ & 1.7 & 70 \\
\hline 0.251 & 7.52 & 1 & 165 & $\mathrm{E}$ & 20 & 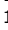 & 155 & $\mathrm{NE}$ & 24 & 5.47 & 118.9 & SE & 14.7 & 207 \\
\hline 0.251 & 17.81 & 1 & 161 & $E$ & 22 & 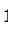 & 156 & $\mathrm{NE}$ & 30 & 8.29 & 144.6 & $\mathrm{SE}$ & 6.5 & 233 \\
\hline 0.251 & 22.59 & 1 & 172 & $\mathrm{E}$ & 24 & 2 & 1.1 & $E$ & 27.9 & 5.55 & 39.4 & $\mathrm{NE}$ & 18.1 & 312 \\
\hline 0.251 & 22.84 & 2 & 1.1 & $\mathrm{E}$ & 27.9 & 3 & 156.1 & $\mathrm{NE}$ & 15.2 & 15.4 & 23.1 & $\mathrm{NE}$ & 11.2 & 116 \\
\hline 0.251 & 24.35 & 5 & 161.2 & $\mathrm{E}$ & 19.4 & 8 & 143.5 & $\mathrm{NE}$ & 20.2 & 6 & 70.4 & $\mathrm{E}$ & 19.4 & 160 \\
\hline 0.251 & 24.6 & 4 & 143.5 & $\mathrm{NE}$ & 20.2 & 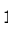 & 165 & $E$ & 29 & 12.44 & 17.3 & $\mathrm{~N}$ & 16.5 & 289 \\
\hline 0.251 & 25.6 & 4 & 163.4 & $\mathrm{E}$ & 29.1 & 5 & 156.9 & $\mathrm{NE}$ & 23.6 & 6.25 & 5.1 & $\mathrm{~N}$ & 11.6 & 98 \\
\hline 0.251 & 26.36 & 3 & 167.3 & $\mathrm{E}$ & 27.8 & 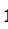 & 166 & $E$ & 27 & 0.99 & 21.7 & $\mathrm{~N}$ & 16.5 & 114 \\
\hline 0.251 & 34.9 & 1 & 165 & $\mathrm{E}$ & 22 & 3 & 162.6 & $\mathrm{E}$ & 24.9 & 3.02 & 146.8 & $\mathrm{SE}$ & 7.2 & 236 \\
\hline 0.251 & 35.15 & 3 & 162.6 & $E$ & 24.9 & 1 & 167 & $E$ & 25 & 1.86 & 70.3 & $\mathrm{E}$ & 24.8 & 342 \\
\hline 0.251 & 35.4 & 1 & 167 & $\mathrm{E}$ & 25 & 3 & 130.3 & $\mathrm{NE}$ & 24.8 & 15.25 & 57.9 & $\mathrm{NE}$ & 23.8 & 150 \\
\hline 0.251 & 35.65 & 3 & 130.3 & $\mathrm{NE}$ & 24.8 & 2 & 144.9 & $\mathrm{NE}$ & 29.4 & 8.07 & 10.1 & $\mathrm{~N}$ & 21.8 & 282 \\
\hline 0.251 & 36.4 & 2 & 138.3 & $\mathrm{NE}$ & 27 & 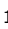 & 143 & $\mathrm{NE}$ & 32 & 5.51 & 162.8 & $\mathrm{~N}$ & 12 & 255 \\
\hline 0.251 & 38.16 & 2 & 160.9 & $\mathrm{E}$ & 28.7 & 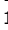 & 106 & $\mathrm{~N}$ & 10 & 24.22 & 178.9 & $\mathrm{~N}$ & 9.6 & 91 \\
\hline 0.251 & 40.92 & 3 & 132.1 & $\mathrm{NE}$ & 15.1 & 2 & 12.6 & $E$ & 17.7 & 16.49 & 64.2 & $\mathrm{NE}$ & 14 & 333 \\
\hline 0.251 & 43.18 & 1 & 175 & $\mathrm{E}$ & 43 & 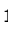 & 153 & $\mathrm{NE}$ & 17 & 27.84 & 5 & $\mathrm{~N}$ & 9.2 & 99 \\
\hline 0.251 & 43.94 & 1 & 160 & $\mathrm{E}$ & 18 & 1 & 157 & $\mathrm{NE}$ & 29 & 11.06 & 152.8 & SE & 2.3 & 242 \\
\hline 0.251 & 47.71 & 9 & 150.4 & $\mathrm{NE}$ & 38.5 & 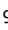 & 139.8 & $\mathrm{NE}$ & 27.8 & 12.19 & 169.7 & $\mathrm{~N}$ & 14.7 & 84 \\
\hline 0.251 & 47.96 & 9 & 139.8 & $\mathrm{NE}$ & 27.8 & 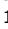 & 139 & $\mathrm{NE}$ & 20 & 7.81 & 141.5 & NW & 0.9 & 52 \\
\hline 0.251 & 50.72 & 1 & 145 & $\mathrm{NE}$ & 26 & 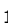 & 147 & $\mathrm{NE}$ & 33 & 7.07 & 153 & NW & 3.9 & 244 \\
\hline 0.251 & 70.31 & 1 & 155 & $\mathrm{NE}$ & 16 & 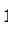 & 143 & $\mathrm{NE}$ & 13 & 4.23 & 13.2 & $\mathrm{~N}$ & 10.1 & 104 \\
\hline 0.251 & 70.56 & 1 & 143 & $\mathrm{NE}$ & 13 & 1 & 141 & $\mathrm{NE}$ & 15 & 2.06 & 128.8 & SE & 3.2 & 218 \\
\hline 0.251 & 70.82 & 1 & 141 & $\mathrm{NE}$ & 15 & 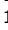 & 162 & $E$ & 19 & 7.27 & 27.6 & $\mathrm{NE}$ & 13.8 & 298 \\
\hline 0.316 & 0.22 & 2 & 158.2 & $\mathrm{E}$ & 25.4 & 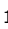 & 156 & $\mathrm{NE}$ & 31 & 5.7 & 147.8 & $\mathrm{SE}$ & 4.9 & 237 \\
\hline 0.316 & 3.38 & 1 & 172 & $\mathrm{E}$ & 35 & 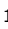 & 179 & $\mathrm{E}$ & 25 & 10.58 & 158.5 & $\mathrm{~s}$ & 9.3 & 66 \\
\hline 0.316 & 4.65 & 2 & 175.4 & $\mathrm{E}$ & 31.4 & 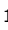 & 160 & $E$ & 29 & 8.09 & 58.3 & $\mathrm{NE}$ & 28.5 & 152 \\
\hline 0.316 & 4.97 & 1 & 160 & $\mathrm{E}$ & 29 & 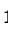 & 168 & $\mathrm{E}$ & 26 & 4.75 & 116.4 & $\mathrm{SE}$ & 20.9 & 23 \\
\hline 0.316 & 5.28 & 1 & 168 & $\mathrm{E}$ & 26 & . & 170 & $\mathrm{E}$ & 36 & 10.05 & 174.1 & $\mathrm{~N}$ & 3 & 265 \\
\hline 0.316 & 6.55 & 1 & 177 & $\mathrm{E}$ & 26 & 8 & 160.6 & $\mathrm{E}$ & 29.9 & 8.6 & 108.5 & $\mathrm{E}$ & 24.4 & 196 \\
\hline 0.316 & 7.5 & 2 & 163.8 & $\mathrm{E}$ & 26 & 1 & 155 & $\mathrm{NE}$ & 24 & 4.21 & 38.8 & $\mathrm{NE}$ & 21.8 & 132 \\
\hline 0.316 & 17.93 & 1 & 161 & $\mathrm{E}$ & 22 & 1 & 156 & $\mathrm{NE}$ & 30 & 8.29 & 144.6 & SE & 6.5 & 233 \\
\hline 0.316 & 18.25 & 1 & 156 & $\mathrm{NE}$ & 30 & 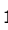 & 158 & $\mathrm{E}$ & 30 & 1 & 67 & $\mathrm{NE}$ & 30 & 337 \\
\hline 0.316 & 22.67 & 2 & 172 & E & 20 & 3 & 171.3 & $\mathrm{E}$ & 24.6 & 4.63 & 168.6 & $\mathrm{~s}$ & 1.2 & 258 \\
\hline 0.316 & 22.99 & 3 & 171.3 & $\mathrm{E}$ & 24.6 & 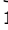 & 154 & $\mathrm{NE}$ & 10 & 15.35 & 1.5 & $\mathrm{~N}$ & 4.7 & 93 \\
\hline 0.316 & 24.26 & 4 & 161.7 & $\mathrm{E}$ & 19.5 & 8 & 144.7 & $\mathrm{NE}$ & 19.6 & 5.66 & 64.3 & $\mathrm{NE}$ & 19.3 & 155 \\
\hline 0.316 & 24.57 & 4 & 144.7 & $\mathrm{NE}$ & 19.6 & & 159.9 & $\mathrm{E}$ & 24.9 & 7.79 & 17.5 & $\mathrm{~N}$ & 15.8 & 289 \\
\hline 0.316 & 25.52 & 1 & 155 & $\mathrm{NE}$ & 22 & & 160.7 & E & 26.5 & 5.08 & 3.1 & $\mathrm{~N}$ & 10.8 & 275 \\
\hline 0.316 & 25.84 & 8 & 160.7 & $\mathrm{E}$ & 26.5 & & 165.1 & $\mathrm{E}$ & 28.7 & 3.04 & 22.5 & $\mathrm{NE}$ & 18.4 & 295 \\
\hline 0.316 & 26.15 & 2 & 165.1 & $\mathrm{E}$ & 28.7 & 2 & 168.9 & $\mathrm{E}$ & 26.5 & 2.87 & 132.5 & $\mathrm{SE}$ & 16.5 & 38 \\
\hline
\end{tabular}




\begin{tabular}{|c|c|c|c|c|c|c|c|c|c|c|c|c|c|c|}
\hline 0.398 & 24.56 & 8 & 153.2 & NE & $19.3 \|$ & 2 & 159.9 & $\mathrm{E}$ & $24.9 \|$ & 6.11 & 179.3 & $\mathrm{~N}$ & 8.8 & 271 \\
\hline 0.398 & 25.76 & 5 & 164.1 & $\mathrm{E}$ & 29.1 & 6 & 158.1 & $\mathrm{E}$ & 24.3 & 5.44 & 8.1 & $\mathrm{~N}$ & 12.7 & 101 \\
\hline 0.398 & 26.16 & 6 & 158.1 & E & 24.3 & 2 & 168.9 & $\mathrm{E}$ & 26.5 & 5.09 & 46.8 & NE & 22.9 & 318 \\
\hline 0.398 & 34.91 & 2 & 160.2 & $E$ & 20.9 & 3 & 165.9 & $\mathrm{E}$ & 26.6 & 6.12 & 3.4 & $\mathrm{~N}$ & 8.6 & 275 \\
\hline 0.398 & 35.31 & 3 & 165.9 & $E$ & 26.6 & 4 & 135.7 & $\mathrm{NE}$ & 25.6 & 13.2 & 56.1 & NE & 25.2 & 148 \\
\hline 0.398 & 35.71 & 4 & 135.7 & NE & 25.6 & 1 & 140 & $\mathrm{NE}$ & 30 & 4.83 & 159.8 & $\mathrm{~N}$ & 11 & 252 \\
\hline 0.398 & 36.11 & 1 & 140 & $\mathrm{NE}$ & 30 & 2 & 138.3 & $\mathrm{NE}$ & 27 & 3.11 & 152.9 & NW & 7.3 & 64 \\
\hline 0.398 & 36.51 & 2 & 138.3 & $\mathrm{NE}$ & 27 & 1 & 143 & $\mathrm{NE}$ & 32 & 5.51 & 162.8 & $\mathrm{~N}$ & 12 & 255 \\
\hline 0.398 & 37.7 & 1 & 90 & $\mathrm{~N}$ & 19 & 1 & 5 & $\mathrm{~S}$ & 35 & 40.69 & 30.2 & NE & 16.6 & 299 \\
\hline 0.398 & 38.1 & 1 & 5 & $\mathrm{~s}$ & 35 & & 124.3 & $\mathrm{NE}$ & 18.7 & 30.01 & 33.9 & NE & 18.7 & 129 \\
\hline 0.398 & 39.69 & 1 & 106 & $\mathrm{~N}$ & 16 & 1 & 107 & $\mathrm{~N}$ & 13 & 3.01 & 101.9 & $\mathrm{E}$ & 1.2 & 12 \\
\hline 0.398 & 40.89 & 2 & 129.7 & NE & 15.2 & 3 & 176.6 & $\mathrm{E}$ & 15.2 & 11.96 & 62.9 & NE & 14 & 332 \\
\hline 0.398 & 47.65 & 7 & 149.1 & NE & 40 & 1 & 142.8 & $\mathrm{NE}$ & 28.1 & 12.46 & 159.7 & $\mathrm{~N}$ & 8.8 & 73 \\
\hline 0.398 & 49.64 & 1 & 150 & NE & 27 & 1 & 143 & $\mathrm{NE}$ & 34 & 7.84 & 122.8 & SE & 13.1 & 210 \\
\hline 0.398 & 50.04 & 1 & 143 & $\mathrm{NE}$ & 34 & 1 & 148 & $\mathrm{NE}$ & 40 & 6.71 & 167.4 & $\mathrm{~N}$ & 15.6 & 262 \\
\hline 0.398 & 50.44 & 1 & 148 & $\mathrm{NE}$ & 40 & 1 & 145 & $\mathrm{NE}$ & 26 & 14.09 & 152.1 & NW & 3.5 & 63 \\
\hline 0.398 & 50.84 & 1 & 145 & NE & 26 & 1 & 147 & $\mathrm{NE}$ & 33 & 7.07 & 153 & NW & 3.9 & 244 \\
\hline 0.398 & 58.8 & 1 & 145 & NE & 23 & 1 & 158 & $\mathrm{E}$ & 22 & 5.06 & 73.7 & $\mathrm{E}$ & 21.9 & 342 \\
\hline 0.398 & 70.35 & 1 & 155 & NE & 16 & 1 & 143 & $\mathrm{NE}$ & 13 & 4.23 & 13.2 & $\mathrm{~N}$ & 10.1 & 104 \\
\hline 0.398 & 70.74 & 1 & 143 & $\mathrm{NE}$ & 13 & 2 & 152.7 & $\mathrm{NE}$ & 16.7 & 4.48 & 0.8 & $\mathrm{~N}$ & 8 & 272 \\
\hline 0.501 & 0.32 & 2 & 158.2 & $\mathrm{E}$ & 25.4 & 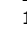 & 156 & $\mathrm{NE}$ & 31 & 5.7 & 147.8 & SE & 4.9 & 237 \\
\hline 0.501 & 3.33 & 1 & 172 & $\mathrm{E}$ & 35 & 1 & 179 & $\mathrm{E}$ & 25 & 10.58 & 158.5 & $\mathrm{~S}$ & 9.3 & 66 \\
\hline 0.501 & 4.83 & 3 & 170.5 & $E$ & 30.4 & 1 & 168 & E & 26 & 4.55 & 2.8 & $\mathrm{~N}$ & 7.1 & 94 \\
\hline 0.501 & 5.33 & 1 & 168 & $\mathrm{E}$ & 26 & 2 & 170 & $\mathrm{E}$ & 36 & 10.05 & 174.1 & $\mathrm{~N}$ & 3 & 265 \\
\hline 0.501 & 6.83 & 5 & 163.5 & E & 29 & 1 & 163 & $\mathrm{E}$ & 32 & 3.04 & 159 & $\mathrm{~s}$ & 2.5 & 248 \\
\hline 0.501 & 7.33 & 1 & 163 & E & 32 & 2 & 159.6 & E & 21.9 & 10.19 & 169.2 & $\mathrm{~N}$ & 3.8 & 80 \\
\hline 0.501 & 9.34 & 1 & 178 & E & 43 & 2 & 161.9 & $E$ & 30.4 & 15.77 & 21.8 & $\mathrm{~N}$ & 20.6 & 119 \\
\hline 0.501 & 17.86 & 1 & 161 & E & 22 & 2 & 157 & $\mathrm{NE}$ & 30 & 8.18 & 147.8 & SE & 5.3 & 237 \\
\hline 0.501 & 22.37 & 1 & 162 & E & 40 & 3 & 178.2 & $\mathrm{E}$ & 26.4 & 16.12 & 141 & SE & 16.7 & 45 \\
\hline 0.501 & 22.87 & 3 & 178.2 & E & 26.4 & 3 & 156.1 & $\mathrm{NE}$ & 15.2 & 13.54 & 20.8 & $\mathrm{~N}$ & 10.8 & 113 \\
\hline 0.501 & 24.38 & 6 & 157.3 & NE & 17.8 & 4 & 152 & $\mathrm{NE}$ & 24.3 & 6.8 & 139.5 & $\mathrm{SE}$ & 5.6 & 228 \\
\hline 0.501 & 25.88 & 9 & 160.1 & $\mathrm{E}$ & 26 & 3 & 167.3 & $\mathrm{E}$ & 27.8 & 3.71 & 42.2 & $\mathrm{NE}$ & 23.3 & 314 \\
\hline 0.501 & 26.38 & 3 & 167.3 & $\mathrm{E}$ & 27.8 & 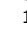 & 166 & $\mathrm{E}$ & 27 & 0.99 & 21.7 & $\mathrm{~N}$ & 16.5 & 114 \\
\hline 0.501 & 31.39 & 1 & 152 & $\mathrm{NE}$ & 29 & 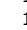 & 160 & $\mathrm{E}$ & 24 & 6.13 & 123.3 & $\mathrm{SE}$ & 14.9 & 30 \\
\hline 0.501 & 34.9 & 2 & 160.2 & $\mathrm{E}$ & 20.9 & 3 & 165.9 & E & 26.6 & 6.12 & 3.4 & $\mathrm{~N}$ & 8.6 & 275 \\
\hline 0.501 & 35.4 & 3 & 165.9 & $\mathrm{E}$ & 26.6 & 5 & 136.7 & NE & 26.5 & 12.94 & 60.7 & NE & 25.8 & 152 \\
\hline 0.501 & 35.9 & 5 & 136.7 & NE & 26.5 & 2 & 138.3 & $\mathrm{NE}$ & 27 & 0.88 & 7.3 & $\mathrm{~N}$ & 21 & 283 \\
\hline 0.501 & 36.4 & 2 & 138.3 & NE & 27 & 1 & 143 & NE & 32 & 5.51 & 162.8 & $\mathrm{~N}$ & 12 & 255 \\
\hline 0.501 & 36.91 & 1 & 143 & $\mathrm{NE}$ & 32 & 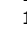 & 90 & $\mathrm{~N}$ & 19 & 25.09 & 176.4 & $\mathrm{~N}$ & 19 & 91 \\
\hline 0.501 & 40.91 & 3 & 132.1 & $\mathrm{NE}$ & 15.1 & 2 & 12.6 & E & 17.7 & 16.49 & 64.2 & $\mathrm{NE}$ & 14 & 333 \\
\hline 0.501 & 43.42 & 2 & 168.4 & $\mathrm{E}$ & 29.6 & 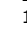 & 160 & $\mathrm{E}$ & 18 & 12.08 & 179.4 & $\mathrm{~N}$ & 6.2 & 91 \\
\hline 0.501 & 43.92 & 1 & 160 & $\mathrm{E}$ & 18 & 1 & 157 & $\mathrm{NE}$ & 29 & 11.06 & 152.8 & SE & 2.3 & 242 \\
\hline 0.501 & 47.93 & 18 & 145.9 & $\mathrm{NE}$ & 33 & 1 & 139 & $\mathrm{NE}$ & 20 & 13.33 & 154.4 & NW & 5.5 & 66 \\
\hline 0.501 & 49.94 & 2 & 146.1 & $\mathrm{NE}$ & 30.5 & 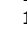 & 148 & $\mathrm{NE}$ & 40 & 9.61 & 152.3 & NW & 3.6 & 243 \\
\hline 0.501 & 50.44 & 1 & 148 & $\mathrm{NE}$ & 40 & 2 & 146.1 & $\mathrm{NE}$ & 29.5 & 10.56 & 151.9 & NW & 3.3 & 63 \\
\hline 0.501 & 58.96 & 1 & 145 & $\mathrm{NE}$ & 23 & 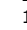 & 158 & $\mathrm{E}$ & 22 & 5.06 & 73.7 & $\mathrm{E}$ & 21.9 & 342 \\
\hline 0.501 & 70.48 & 2 & 149.6 & $\mathrm{NE}$ & 14.4 & 3 & 152.7 & $\mathrm{NE}$ & 16.7 & 2.46 & 170.3 & $\mathrm{~N}$ & 5.2 & 261 \\
\hline 0.631 & 5.04 & 4 & 170 & $\mathrm{E}$ & 29.3 & 2 & 170 & $\mathrm{E}$ & 36 & 6.71 & 170.1 & $\mathrm{~N}$ & 0.1 & 260 \\
\hline 0.631 & 6.93 & 5 & 163.5 & $\mathrm{E}$ & 29 & 2 & 163.8 & $\mathrm{E}$ & 26 & 2.98 & 161.5 & $\mathrm{~s}$ & 1.1 & 71 \\
\hline 0.631 & 7.57 & 2 & 163.8 & $\mathrm{E}$ & 26 & 1 & 155 & $\mathrm{NE}$ & 24 & 4.21 & 38.8 & NE & 21.8 & 132 \\
\hline 0.631 & 9.46 & 1 & 178 & $\mathrm{E}$ & 43 & 2 & 161.9 & $\mathrm{E}$ & 30.4 & 15.77 & 21.8 & $\mathrm{~N}$ & 20.6 & 119 \\
\hline 0.631 & 11.35 & 1 & 159 & E & 25 & 1 & 165 & $\mathrm{E}$ & 36 & 11.4 & 175.5 & $\mathrm{~N}$ & 7.5 & 267 \\
\hline 0.631 & 18.29 & 2 & 158.1 & $\mathrm{E}$ & 26 & 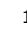 & 158 & $\mathrm{E}$ & 30 & 4.02 & 157.2 & $\mathrm{SE}$ & 0.4 & 247 \\
\hline 0.631 & 21.45 & 1 & 128 & $\mathrm{NE}$ & 12 & 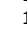 & 162 & $\mathrm{E}$ & 40 & 30.67 & 172.2 & $\mathrm{~N}$ & 8.4 & 263 \\
\hline 0.631 & 22.08 & 1 & 162 & E & 40 & 2 & 172 & E & 20 & 20.55 & 154.5 & SE & 6.2 & 62 \\
\hline 0.631 & 22.71 & 2 & 172 & $\mathrm{E}$ & 20 & 4 & 168.9 & $\mathrm{E}$ & 20.6 & 1.24 & 111.4 & $\mathrm{E}$ & 17.6 & 200 \\
\hline 0.631 & 24.6 & 9 & 153.2 & $\mathrm{NE}$ & 19.5 & 1 & 165 & E & 29 & 10.63 & 4.2 & $\mathrm{~N}$ & 10.4 & 276 \\
\hline 0.631 & 25.23 & 1 & 165 & $\mathrm{E}$ & 29 & 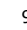 & 160.1 & $\mathrm{E}$ & 26 & 3.76 & 16.2 & $\mathrm{~N}$ & 16 & 110 \\
\hline 0.631 & 25.86 & 9 & 160.1 & $\mathrm{E}$ & 26 & 4 & 167 & $\mathrm{E}$ & 27.6 & 3.48 & 43.8 & NE & 23.6 & 316 \\
\hline 0.631 & 31.54 & 1 & 152 & $\mathrm{NE}$ & 29 & 1 & 160 & $\mathrm{E}$ & 24 & 6.13 & 123.3 & $\mathrm{SE}$ & 14.9 & 30 \\
\hline 0.631 & 35.33 & 5 & 163.9 & $\mathrm{E}$ & 24.3 & 5 & 136.7 & $\mathrm{NE}$ & 26.5 & 11.78 & 71.7 & $\mathrm{E}$ & 24.3 & 162 \\
\hline 0.631 & 35.96 & 5 & 136.7 & $\mathrm{NE}$ & 26.5 & 3 & 140.1 & $\mathrm{NE}$ & 28.7 & 2.7 & 170.3 & $\mathrm{~N}$ & 15.4 & 264 \\
\hline 0.631 & 37.85 & 1 & 90 & $\mathrm{~N}$ & 19 & 3 & 152.5 & $\mathrm{NE}$ & 21.1 & 20.57 & 25.8 & NE & 17.2 & 295 \\
\hline 0.631 & 39.74 & 1 & 106 & $\mathrm{~N}$ & 16 & 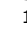 & 107 & $\mathrm{~N}$ & 13 & 3.01 & 101.9 & $\mathrm{E}$ & 1.2 & 12 \\
\hline 0.631 & 40.38 & 1 & 107 & $\mathrm{~N}$ & 13 & 3 & 132.1 & $\mathrm{NE}$ & 15.1 & 6.39 & 10.5 & $\mathrm{~N}$ & 12.9 & 280 \\
\hline 0.631 & 41.01 & 3 & 132.1 & $\mathrm{NE}$ & 15.1 & 2 & 12.6 & $\mathrm{E}$ & 17.7 & 16.49 & 64.2 & $\mathrm{NE}$ & 14 & 333 \\
\hline 0.631 & 43.53 & 2 & 168.4 & E & 29.6 & 2 & 158.2 & E & 23.5 & 7.63 & 17.3 & $\mathrm{~N}$ & 15.3 & 111 \\
\hline 0.631 & 47.95 & 18 & 145.9 & $\mathrm{NE}$ & 33 & 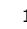 & 139 & $\mathrm{NE}$ & 20 & 13.33 & 154.4 & NW & 5.5 & 66 \\
\hline 0.631 & 49.84 & 2 & 146.1 & $\mathrm{NE}$ & 30.5 & 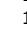 & 148 & $\mathrm{NE}$ & 40 & 9.61 & 152.3 & NW & 3.6 & 243 \\
\hline 0.631 & 50.47 & 1 & 148 & $\mathrm{NE}$ & 40 & 2 & 146.1 & $\mathrm{NE}$ & 29.5 & 10.56 & 151.9 & NW & 3.3 & 63 \\
\hline 0.631 & 70.66 & 2 & 149.6 & $\mathrm{NE}$ & 14.4 & 2 & 152.7 & $\mathrm{NE}$ & 16.7 & 2.46 & 170.3 & $\mathrm{~N}$ & 5.2 & 261 \\
\hline 0.631 & 71.29 & 2 & 152.7 & $\mathrm{NE}$ & 16.7 & 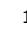 & 175 & $\mathrm{E}$ & 28 & 13.92 & 19.2 & $\mathrm{~N}$ & 12.3 & 290 \\
\hline 0.794 & 0.76 & 3 & 157.4 & $\mathrm{NE}$ & 27.3 & 1 & 159 & $\mathrm{E}$ & 19 & 8.29 & 154.1 & SE & 1.7 & 64 \\
\hline 0.794 & 3.94 & 2 & 175 & E & 30 & 2 & 175.4 & E & 31.4 & 1.45 & 3.1 & $\mathrm{~N}$ & 4.7 & 273 \\
\hline 0.794 & 4.73 & 2 & 175.4 & $\mathrm{E}$ & 31.4 & 4 & 167.3 & $\mathrm{E}$ & 31.6 & 4.27 & 85.4 & $\mathrm{E}$ & 31.4 & 175 \\
\hline 0.794 & 7.12 & 5 & 163.5 & E & 29 & 3 & 161 & E & 25.3 & 3.88 & 177.7 & $\mathrm{~N}$ & 7.8 & 90 \\
\hline 0.794 & 9.5 & 1 & 178 & E & 43 & 2 & 161.9 & E & 30.4 & 15.77 & 21.8 & $\mathrm{~N}$ & 20.6 & 119 \\
\hline 0.794 & 11.88 & 1 & 159 & $\mathrm{E}$ & 25 & 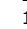 & 165 & $\mathrm{E}$ & 36 & 11.4 & 175.5 & $\mathrm{~N}$ & 7.5 & 267 \\
\hline 0.794 & 18.24 & 2 & 158.1 & $\mathrm{E}$ & 26 & 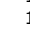 & 158 & $\mathrm{E}$ & 30 & 4.02 & 157.2 & $\mathrm{SE}$ & 0.4 & 247 \\
\hline
\end{tabular}




\begin{tabular}{|c|c|c|c|c|c|c|c|c|c|c|c|c|c|c|}
\hline 1 & 11.91 & 1 & 159 & $\mathrm{E}$ & 25 & 1 & 165 & $\mathrm{E}$ & 36 & 11.4 & 175.5 & $\mathrm{~N}$ & 7.5 & 267 \\
\hline 1 & 12.91 & 1 & 165 & $\mathrm{E}$ & 36 & 2 & 165.5 & $\mathrm{E}$ & 38 & 2 & 172.2 & $\mathrm{~N}$ & 5.2 & 264 \\
\hline 1 & 16.91 & 2 & 171.6 & E & 25.3 & 1 & 161 & E & 22 & 5.37 & 36.3 & $\mathrm{NE}$ & 18.4 & 129 \\
\hline 1 & 17.91 & 1 & 161 & E & 22 & 2 & 157 & $\mathrm{NE}$ & 30 & 8.18 & 147.8 & SE & 5.3 & 237 \\
\hline 1 & 21.91 & 1 & 128 & $\mathrm{NE}$ & 12 & 5 & 169.6 & $\mathrm{E}$ & 25.5 & 18.2 & 13.6 & $\mathrm{~N}$ & 11 & 284 \\
\hline 1 & 22.91 & 5 & 169.6 & $\mathrm{E}$ & 25.5 & 2 & 163.3 & E & 17.9 & 7.89 & 2.6 & $\mathrm{~N}$ & 6.1 & 94 \\
\hline 1 & 23.91 & 2 & 163.3 & $\mathrm{E}$ & 17.9 & 10 & 154.8 & $\mathrm{NE}$ & 20.4 & 3.74 & 112.5 & $\mathrm{E}$ & 14.1 & 201 \\
\hline 1 & 24.91 & 10 & 154.8 & $\mathrm{NE}$ & 20.4 & 9 & 160.1 & $\mathrm{E}$ & 26 & 5.95 & 176.4 & $\mathrm{~N}$ & 7.8 & 268 \\
\hline 1 & 25.91 & 9 & 160.1 & $E$ & 26 & 4 & 167 & E & 27.6 & 3.48 & 43.8 & $\mathrm{NE}$ & 23.6 & 316 \\
\hline 1 & 26.91 & 4 & 167 & $\mathrm{E}$ & 27.6 & 1 & 154 & $\mathrm{NE}$ & 30 & 6.7 & 94.2 & $E$ & 26.5 & 182 \\
\hline 1 & 34.91 & 2 & 160.2 & $\mathrm{E}$ & 20.9 & 8 & 147.6 & $\mathrm{NE}$ & 25.8 & 6.98 & 110.6 & $\mathrm{E}$ & 16.2 & 199 \\
\hline 1 & 35.91 & 8 & 147.6 & $\mathrm{NE}$ & 25.8 & 3 & 140.1 & $\mathrm{NE}$ & 28.7 & 4.49 & 96.6 & $\mathrm{E}$ & 20.6 & 184 \\
\hline 1 & 36.9 & 3 & 140.1 & $\mathrm{NE}$ & 28.7 & 1 & 90 & $\mathrm{~N}$ & 19 & 21.59 & 179 & $\mathrm{~N}$ & 19 & 93 \\
\hline 1 & 37.9 & 1 & 90 & $\mathrm{~N}$ & 19 & 3 & 152.5 & $\mathrm{NE}$ & 21.1 & 20.57 & 25.8 & $\mathrm{NE}$ & 17.2 & 295 \\
\hline 1 & 38.9 & 3 & 152.5 & $\mathrm{NE}$ & 21.1 & 2 & 106.4 & $\mathrm{~N}$ & $14.5 \|$ & 15.03 & 14.4 & $\mathrm{~N}$ & 14.5 & 107 \\
\hline 1 & 39.9 & 2 & 106.4 & $\mathrm{~N}$ & 14.5 & 3 & 132.1 & $\mathrm{NE}$ & 15.1 & 6.53 & 24.1 & $\mathrm{NE}$ & 14.4 & 294 \\
\hline 1 & 40.9 & 3 & 132.1 & $\mathrm{NE}$ & 15.1 & 2 & 12.6 & $\mathrm{E}$ & 17.7 & 16.49 & 64.2 & $\mathrm{NE}$ & 14 & 333 \\
\hline 1 & 43.9 & 3 & 166.2 & $E$ & 25.4 & 1 & 157 & $\mathrm{NE}$ & 29 & 5.53 & 115.4 & SE & 20.2 & 203 \\
\hline 1 & 44.9 & 1 & 157 & $\mathrm{NE}$ & 29 & 1 & 11 & $\mathrm{~S}$ & 32 & 17.31 & 72.9 & $\mathrm{E}$ & 28.9 & 342 \\
\hline 1 & 47.9 & 17 & 147.3 & $\mathrm{NE}$ & 34.1 & 2 & 119.5 & $\mathrm{NE}$ & 18.9 & 19.22 & 170.6 & $\mathrm{~N}$ & 14.9 & 85 \\
\hline 1 & 48.9 & 2 & 119.5 & $\mathrm{NE}$ & 18.9 & 2 & 146.1 & $\mathrm{NE}$ & 30.5 & 15.76 & 174.8 & $\mathrm{~N}$ & 15.8 & 266 \\
\hline 1 & 49.9 & 2 & 146.1 & $\mathrm{NE}$ & 30.5 & 3 & 146.9 & $\mathrm{NE}$ & 33 & 2.57 & 153.7 & NW & 4.4 & 246 \\
\hline 1 & 58.9 & 1 & 145 & $\mathrm{NE}$ & 23 & 1 & 158 & $\mathrm{E}$ & 22 & 5.06 & 73.7 & $\mathrm{E}$ & 21.9 & 342 \\
\hline 1 & 70.9 & 3 & 146.7 & $\mathrm{NE}$ & 14.6 & 2 & 169.7 & E & 23.4 & 11.39 & 17.5 & $\mathrm{~N}$ & 11.4 & 288 \\
\hline 1.259 & 1.03 & 3 & 157.4 & $\mathrm{NE}$ & 27.3 & 1 & 159 & E & 19 & 8.29 & 154.1 & SE & 1.7 & 64 \\
\hline 1.259 & 2.28 & 1 & 159 & $\mathrm{E}$ & 19 & 1 & 172 & $\mathrm{E}$ & 35 & 16.97 & 4 & $\mathrm{~N}$ & 8.3 & 275 \\
\hline 1.259 & 3.54 & 1 & 172 & E & 35 & 4 & 172.4 & E & 29 & 6.01 & 170.5 & $\mathrm{~s}$ & 1 & 80 \\
\hline 1.259 & 4.8 & 4 & 172.4 & E & 29 & 3 & 169.5 & E & 32.6 & 3.93 & 151.4 & SE & 11.2 & 239 \\
\hline 1.259 & 6.06 & 3 & 169.5 & $\mathrm{E}$ & 32.6 & 6 & 163.4 & E & 29.5 & 4.42 & 26.8 & $\mathrm{NE}$ & 21.2 & 122 \\
\hline 1.259 & 7.32 & 6 & 163.4 & $\mathrm{E}$ & 29.5 & 2 & 159.6 & $\mathrm{E}$ & 21.9 & 7.74 & 172.8 & $\mathrm{~N}$ & 5.3 & 84 \\
\hline 1.259 & 8.58 & 2 & 159.6 & $\mathrm{E}$ & 21.9 & 3 & 168.4 & $\mathrm{E}$ & 34.3 & 13.04 & 0.6 & $\mathrm{~N}$ & 8.2 & 272 \\
\hline 1.259 & 14.87 & 2 & 165.5 & E & 38 & 1 & 4 & s & 30 & 12.98 & 127.3 & SE & 25.8 & 31 \\
\hline 1.259 & 16.13 & 1 & 4 & $\mathrm{~S}$ & 30 & 1 & 155 & $\mathrm{NE}$ & 22 & 14.81 & 45.2 & $\mathrm{NE}$ & 20.8 & 139 \\
\hline 1.259 & 17.39 & 1 & 155 & $\mathrm{NE}$ & 22 & 3 & 158.1 & $\mathrm{E}$ & 27.3 & 5.48 & 169 & $\mathrm{~N}$ & 5.6 & 260 \\
\hline 1.259 & 22.43 & 2 & 153.9 & $\mathrm{NE}$ & 25.3 & 6 & 169.9 & $\mathrm{E}$ & 20.4 & 7.87 & 112.1 & $\mathrm{E}$ & 17.5 & 19 \\
\hline 1.259 & 23.69 & 6 & 169.9 & $\mathrm{E}$ & 20.4 & 10 & 154.8 & $\mathrm{NE}$ & 20.4 & 5.25 & 72.6 & $\mathrm{E}$ & 20.2 & 163 \\
\hline 1.259 & 24.95 & 10 & 154.8 & $\mathrm{NE}$ & 20.4 & 12 & 162 & $E$ & 26.4 & 6.64 & 2.1 & $\mathrm{~N}$ & 9.7 & 274 \\
\hline 1.259 & 26.2 & 12 & 162 & $E$ & 26.4 & 2 & 159.7 & E & 28.4 & 2.23 & 135.1 & SE & 12.7 & 223 \\
\hline 1.259 & 31.24 & 1 & 152 & $\mathrm{NE}$ & 29 & 1 & 160 & E & 24 & 6.13 & 123.3 & SE & 14.9 & 30 \\
\hline 1.259 & 35.02 & 3 & 164.2 & $E$ & 23.9 & 7 & 144 & $\mathrm{NE}$ & 25.6 & 8.6 & 76.3 & $\mathrm{E}$ & 23.9 & 166 \\
\hline 1.259 & 36.28 & 7 & 144 & $\mathrm{NE}$ & 25.6 & 4 & 131.5 & $\mathrm{NE}$ & 25 & 5.34 & 41.4 & $\mathrm{NE}$ & 25 & 132 \\
\hline 1.259 & 37.53 & 4 & 131.5 & $\mathrm{NE}$ & 25 & 3 & 152.5 & $\mathrm{NE}$ & 21.1 & 9.02 & 79 & $E$ & 20.3 & 346 \\
\hline 1.259 & 38.79 & 3 & 152.5 & $\mathrm{NE}$ & 21.1 & 2 & 106.4 & $\mathrm{~N}$ & 14.5 & 15.03 & 14.4 & $\mathrm{~N}$ & 14.5 & 107 \\
\hline 1.259 & 40.05 & 2 & 106.4 & $\mathrm{~N}$ & 14.5 & 5 & 157.7 & E & 14 & 12.23 & 44.2 & $\mathrm{NE}$ & 12.9 & 313 \\
\hline 1.259 & 43.83 & 2 & 168.4 & E & 29.6 & 2 & 158.2 & $\mathrm{E}$ & 23.5 & 7.63 & 17.3 & $\mathrm{~N}$ & 15.3 & 111 \\
\hline 1.259 & 45.09 & 2 & 158.2 & $\mathrm{E}$ & 23.5 & 1 & 11 & $\mathrm{~s}$ & 32 & 17.2 & 53.2 & $\mathrm{NE}$ & 22.8 & 324 \\
\hline 1.259 & 46.35 & 1 & 11 & s & 32 & 5 & 159.4 & E & 40.1 & 20.05 & 113 & SE & 31.4 & 201 \\
\hline 1.259 & 47.61 & 5 & 159.4 & E & 40.1 & 14 & 139.3 & $\mathrm{NE}$ & 30 & 15.25 & 12.9 & $\mathrm{~N}$ & 25 & 110 \\
\hline 1.259 & 48.86 & 14 & 139.3 & $\mathrm{NE}$ & 30 & 2 & 146.1 & $\mathrm{NE}$ & 30.5 & 3.45 & 44.8 & $\mathrm{NE}$ & 30 & 314 \\
\hline 1.259 & 50.12 & 2 & 146.1 & $\mathrm{NE}$ & 30.5 & 3 & 146.9 & $\mathrm{NE}$ & 33 & 2.57 & 153.7 & NW & 4.4 & 246 \\
\hline 1.259 & 58.94 & 1 & 145 & $\mathrm{NE}$ & 23 & 1 & 158 & $E$ & 22 & 5.06 & 73.7 & $\mathrm{E}$ & 21.9 & 342 \\
\hline 1.259 & 70.27 & 1 & 155 & $\mathrm{NE}$ & 16 & 3 & 150 & $\mathrm{NE}$ & 15.4 & 1.47 & 39.5 & $\mathrm{NE}$ & 14.5 & 129 \\
\hline 1.259 & 71.53 & 3 & 150 & $\mathrm{NE}$ & 15.4 & 1 & 175 & $E$ & 28 & 15.35 & 17.6 & $\mathrm{~N}$ & 11.5 & 288 \\
\hline 1.259 & 72.78 & 1 & 175 & $E$ & 28 & 1 & 60 & NW & 2 & 28.9 & 178.3 & $\mathrm{~N}$ & 1.8 & 89 \\
\hline 1.585 & 1.24 & 3 & 157.4 & $\mathrm{NE}$ & 27.3 & 1 & 159 & $\mathrm{E}$ & 19 & 8.29 & 154.1 & SE & 1.7 & 64 \\
\hline 1.585 & 2.83 & 1 & 159 & E & 19 & 2 & 175 & E & 30 & 12.71 & 16.1 & $\mathrm{~N}$ & 11.7 & 288 \\
\hline 1.585 & 4.41 & 2 & 175 & E & 30 & 6 & 170 & E & 31.5 & 2.98 & 117.6 & SE & 25.9 & 203 \\
\hline 1.585 & 6 & 6 & 170 & E & 31.5 & 8 & 162.6 & $\mathrm{E}$ & 27.6 & 5.33 & 25.2 & $\mathrm{NE}$ & 19.4 & 120 \\
\hline 1.585 & 7.58 & 8 & 162.6 & $\mathrm{E}$ & 27.6 & 1 & 178 & $\mathrm{E}$ & 43 & 17.69 & 15.9 & $\mathrm{~N}$ & 16 & 289 \\
\hline 1.585 & 9.17 & 1 & 178 & $\mathrm{E}$ & 43 & 2 & 161.9 & $\mathrm{E}$ & 30.4 & 15.77 & 21.8 & $\mathrm{~N}$ & 20.6 & 119 \\
\hline 1.585 & 10.75 & 2 & 161.9 & E & 30.4 & 2 & 162.5 & E & 30.5 & 0.31 & 54.4 & $\mathrm{NE}$ & 29.2 & 324 \\
\hline 1.585 & 12.34 & 2 & 162.5 & E & 30.5 & 2 & 165.5 & E & 38 & 7.69 & 174.6 & $\mathrm{~N}$ & 7 & 266 \\
\hline 1.585 & 17.09 & 2 & 171.6 & $\mathrm{E}$ & 25.3 & 3 & 158.1 & $\mathrm{E}$ & 27.3 & 6.32 & 95.5 & $\mathrm{E}$ & 24.6 & 184 \\
\hline 1.585 & 21.85 & 1 & 128 & $\mathrm{NE}$ & 12 & 7 & 168.2 & $\mathrm{E}$ & 23.2 & 15.91 & 15.4 & $\mathrm{~N}$ & 11.1 & 286 \\
\hline 1.585 & 23.43 & 7 & 168.2 & $\mathrm{E}$ & 23.2 & 10 & 154.8 & $\mathrm{NE}$ & 20.4 & 5.66 & 40.5 & $\mathrm{NE}$ & 18.7 & 133 \\
\hline 1.585 & 25.02 & 10 & 154.8 & $\mathrm{NE}$ & 20.4 & 13 & 162.3 & $E$ & 26.5 & 6.73 & 3 & $\mathrm{~N}$ & 10 & 274 \\
\hline 1.585 & 26.6 & 13 & 162.3 & $E$ & 26.5 & 1 & 154 & $\mathrm{NE}$ & 30 & 5.3 & 113.7 & SE & 20.5 & 200 \\
\hline 1.585 & 31.36 & 1 & 152 & $\mathrm{NE}$ & 29 & 1 & 160 & $E$ & 24 & 6.13 & 123.3 & SE & 14.9 & 30 \\
\hline 1.585 & 36.11 & 10 & 149.8 & $\mathrm{NE}$ & 24.8 & 4 & 131.5 & $\mathrm{NE}$ & 25 & 7.67 & 52.7 & $\mathrm{NE}$ & 24.6 & 143 \\
\hline 1.585 & 37.7 & 4 & 131.5 & $\mathrm{NE}$ & 25 & 3 & 152.5 & $\mathrm{NE}$ & 21.1 & 9.02 & 79 & $\mathrm{E}$ & 20.3 & 346 \\
\hline 1.585 & 39.28 & 3 & 152.5 & $\mathrm{NE}$ & 21.1 & 4 & 118.3 & $\mathrm{NE}$ & 14.5 & 12.1 & 12.8 & $\mathrm{~N}$ & 14 & 105 \\
\hline 1.585 & 40.87 & 4 & 118.3 & $\mathrm{NE}$ & 14.5 & 3 & 176.6 & $\mathrm{E}$ & 15.2 & 14.37 & 55 & $\mathrm{NE}$ & 13 & 324 \\
\hline 1.585 & 42.45 & 3 & 176.6 & $\mathrm{E}$ & 15.2 & 4 & 163.6 & $\mathrm{E}$ & 26.3 & 11.93 & 148.7 & $\mathrm{SE}$ & 7.2 & 238 \\
\hline 1.585 & 47.21 & 1 & 11 & $\mathrm{~s}$ & 32 & 19 & 145.6 & $\mathrm{NE}$ & 32.3 & 23.69 & 79 & $\mathrm{E}$ & 30.1 & 172 \\
\hline 1.585 & 48.79 & 19 & 145.6 & $\mathrm{NE}$ & 32.3 & 3 & 146.9 & $\mathrm{NE}$ & 33.6 & 1.54 & 168.8 & $\mathrm{~N}$ & 14 & 265 \\
\hline 1.585 & 50.38 & 3 & 146.9 & $\mathrm{NE}$ & 33.6 & 2 & 146.1 & $\mathrm{NE}$ & 29.5 & 4.16 & 151.1 & NW & 2.8 & 62 \\
\hline 1.585 & 70.98 & 4 & 151.3 & $\mathrm{NE}$ & 15.6 & 1 & 175 & $\mathrm{E}$ & 28 & 15 & 17.1 & $\mathrm{~N}$ & 11.3 & 288 \\
\hline 1.585 & 72.57 & 1 & 175 & $\mathrm{E}$ & 28 & 1 & 60 & NW & 2 & 28.9 & 178.3 & $\mathrm{~N}$ & 1.8 & 89 \\
\hline 1.995 & 1.99 & 4 & 157.7 & $\mathrm{E}$ & 25.2 & 2 & 175 & $E$ & 30 & 9.26 & 42.7 & $\mathrm{NE}$ & 23.1 & 315 \\
\hline
\end{tabular}




\begin{tabular}{|c|c|c|c|c|c|c|c|c|c|c|c|c|c|c|}
\hline 2.512 & 21.83 & 1 & 128 & $\mathrm{NE}$ & 12 & 1 & 165.4 & E & 21.5 & 13.92 & 15.3 & $\mathrm{~N}$ & 11.1 & 286 \\
\hline 2.512 & 24.35 & 12 & 165.4 & $\mathrm{E}$ & 21.5 & 1 & 159.2 & E & 25 & 4.31 & 129.5 & SE & 13 & 218 \\
\hline 2.512 & 26.86 & 18 & 159.2 & $\mathrm{E}$ & 25 & 1 & 154 & $\mathrm{NE}$ & 30 & 5.51 & 133.5 & SE & 11.4 & 221 \\
\hline 2.512 & 29.37 & 1 & 154 & NE & 30 & 2 & 155.6 & $\mathrm{NE}$ & 26.4 & 3.64 & 143.9 & $\mathrm{SE}$ & 5.8 & 53 \\
\hline 2.512 & 36.9 & 13 & 147.3 & $\mathrm{NE}$ & 25.6 & 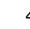 & 137.9 & $\mathrm{NE}$ & 18.5 & 7.92 & 167.4 & $\mathrm{~N}$ & 9.4 & 79 \\
\hline 2.512 & 39.42 & 4 & 137.9 & $\mathrm{NE}$ & 18.5 & 7 & 142.8 & $\mathrm{NE}$ & 13 & 5.6 & 127 & SE & 3.6 & 37 \\
\hline 2.512 & 41.93 & 7 & 142.8 & $\mathrm{NE}$ & 13 & 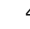 & 163.6 & E & 26.3 & 14.79 & 0.2 & $\mathrm{~N}$ & 8 & 271 \\
\hline 2.512 & 44.44 & 4 & 163.6 & $\mathrm{E}$ & 26.3 & 1 & 11 & $\mathrm{~s}$ & 32 & 14.36 & 61.7 & $\mathrm{NE}$ & 25.8 & 333 \\
\hline 2.512 & 46.95 & 1 & 11 & $\mathrm{~s}$ & 32 & 1 & 145.6 & $\mathrm{NE}$ & 32.3 & 23.69 & 79 & $\mathrm{E}$ & 30.1 & 172 \\
\hline 2.512 & 49.46 & 19 & 145.6 & $\mathrm{NE}$ & 32.3 & 5 & 146.6 & $\mathrm{NE}$ & 32 & 0.6 & 89 & $\mathrm{E}$ & 27.8 & 355 \\
\hline 2.512 & 72.07 & 5 & 158.4 & $\mathrm{E}$ & 17.7 & 1 & 60 & NW & 2 & 18.11 & 164.4 & $\mathrm{~N}$ & 1.9 & 75 \\
\hline 3.162 & 2.12 & 4 & 157.7 & $\mathrm{E}$ & 25.2 & 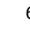 & 171.7 & E & 29.5 & 7.69 & 37.8 & $\mathrm{NE}$ & 22.2 & 310 \\
\hline 3.162 & 5.28 & 6 & 171.7 & $\mathrm{E}$ & 29.5 & 1 & 164.4 & $\mathrm{E}$ & 29.2 & 3.56 & 72.8 & $\mathrm{E}$ & 29.2 & 164 \\
\hline 3.162 & 8.44 & 10 & 164.4 & $E$ & 29.2 & 4 & 166.4 & $\mathrm{E}$ & 31.9 & 2.87 & 3.8 & $\mathrm{~N}$ & 10.5 & 276 \\
\hline 3.162 & 11.61 & 4 & 166.4 & $\mathrm{E}$ & 31.9 & 3 & 165.3 & $\mathrm{E}$ & 37.3 & 5.46 & 160.5 & $\mathrm{~S}$ & 3.7 & 249 \\
\hline 3.162 & 14.77 & 3 & 165.3 & $\mathrm{E}$ & 37.3 & 3 & 168.3 & E & 24.1 & 13.3 & 161.1 & s & 3.2 & 70 \\
\hline 3.162 & 17.93 & 3 & 168.3 & $\mathrm{E}$ & 24.1 & 2 & 157 & $\mathrm{NE}$ & 30 & 7.81 & 124.7 & SE & 17.1 & 212 \\
\hline 3.162 & 21.09 & 2 & 157 & $\mathrm{NE}$ & 30 & 1 & 164.2 & $\mathrm{E}$ & 20.7 & 9.78 & 143.8 & $\mathrm{SE}$ & 7.5 & 52 \\
\hline 3.162 & 24.26 & 12 & 164.2 & $\mathrm{E}$ & 20.7 & 2 & 158.8 & E & 25 & 4.74 & 137.3 & SE & 9.7 & 226 \\
\hline 3.162 & 33.74 & 2 & 155.6 & NE & 26.4 & 1 & 147.3 & $\mathrm{NE}$ & 25.6 & 3.76 & 47.2 & $\mathrm{NE}$ & 25.3 & 139 \\
\hline 3.162 & 36.91 & 13 & 147.3 & $\mathrm{NE}$ & 25.6 & 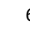 & 128 & $\mathrm{NE}$ & 16.5 & 11.29 & 173.5 & $\mathrm{~N}$ & 12 & 86 \\
\hline 3.162 & 40.07 & 6 & 128 & $\mathrm{NE}$ & 16.5 & 7 & 162.2 & $\mathrm{E}$ & 18.1 & 10.14 & 46.5 & $\mathrm{NE}$ & 16.4 & 316 \\
\hline 3.162 & 43.23 & 7 & 162.2 & $\mathrm{E}$ & 18.1 & 3 & 171.1 & $\mathrm{E}$ & 25.4 & 8.05 & 9.4 & $\mathrm{~N}$ & 8.5 & 281 \\
\hline 3.162 & 46.39 & 3 & 171.1 & E & 25.4 & 1 & 145.6 & $\mathrm{NE}$ & 32.3 & 13.95 & 100.2 & $\mathrm{E}$ & 24.2 & 189 \\
\hline 3.162 & 49.55 & 19 & 145.6 & $\mathrm{NE}$ & 32.3 & 5 & 146.6 & $\mathrm{NE}$ & 32 & 0.6 & 89 & E & 27.8 & 355 \\
\hline 3.162 & 59.04 & 1 & 145 & $\mathrm{NE}$ & 23 & 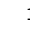 & 158 & E & 22 & 5.06 & 73.7 & $\mathrm{E}$ & 21.9 & 342 \\
\hline 3.162 & 68.53 & 1 & 152 & $\mathrm{NE}$ & 22 & 2 & 151.3 & $\mathrm{NE}$ & 15.6 & 6.43 & 153.6 & NW & 0.7 & 64 \\
\hline 3.162 & 71.69 & 4 & 151.3 & $\mathrm{NE}$ & 15.6 & 2 & 171 & $E$ & 13.6 & 5.32 & 93.1 & E & 13.3 & 3 \\
\hline 3.981 & 3.07 & 4 & 157.7 & $\mathrm{E}$ & 25.2 & 1 & 168.4 & E & 30.2 & 7.05 & 24.3 & $\mathrm{NE}$ & 18.9 & 297 \\
\hline 3.981 & 7.05 & 13 & 168.4 & E & 30.2 & $\epsilon$ & 165.1 & E & 29.7 & 1.71 & 56.2 & $\mathrm{NE}$ & 28.3 & 150 \\
\hline 3.981 & 11.03 & 6 & 165.1 & E & 29.7 & 2 & 164.2 & E & 34.2 & 4.58 & 159.3 & $\mathrm{~s}$ & 3.3 & 249 \\
\hline 3.981 & 15.01 & 4 & 164.2 & E & 34.2 & 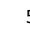 & 163.2 & $\mathrm{E}$ & 26.4 & 7.9 & \begin{tabular}{|l|}
166.7 \\
\end{tabular} & $\mathrm{~N}$ & 1.8 & 77 \\
\hline 3.981 & 18.99 & 5 & 163.2 & E & 26.4 & $\epsilon$ & 166 & E & 22.6 & 3.91 & 148.6 & SE & 7.1 & 57 \\
\hline 3.981 & 22.97 & 6 & 166 & E & 22.6 & 2 & 159.8 & E & 23.3 & 2.54 & 90.1 & $\mathrm{E}$ & 22 & 179 \\
\hline 3.981 & 26.95 & 25 & 159.8 & E & 23.3 & 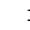 & 154 & $\mathrm{NE}$ & 30 & 7.15 & 137.7 & SE & 9.2 & 226 \\
\hline 3.981 & 30.93 & 1 & 154 & $\mathrm{NE}$ & 30 & 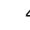 & 157.7 & E & 23.7 & 6.55 & 142.6 & SE & 6.5 & 51 \\
\hline 3.981 & 34.91 & 4 & 157.7 & $\mathrm{E}$ & 23.7 & 1 & 144.1 & $\mathrm{NE}$ & 24.5 & 5.62 & 70.7 & $\mathrm{E}$ & 23.6 & 160 \\
\hline 3.981 & 38.9 & 15 & 144.1 & $\mathrm{NE}$ & 24.5 & i & 142.8 & $\mathrm{NE}$ & 13 & 11.5 & 145.4 & NW & 0.6 & 56 \\
\hline 3.981 & 42.88 & 7 & 142.8 & $\mathrm{NE}$ & 13 & 5 & 170 & $\mathrm{E}$ & 27 & 16.44 & 9.2 & $\mathrm{~N}$ & 9.5 & 280 \\
\hline 3.981 & 46.86 & 5 & 170 & $E$ & 27 & 2 & 145.8 & $\mathrm{NE}$ & 32.2 & 12.91 & 93.8 & $E$ & 26.3 & 183 \\
\hline 3.981 & 50.84 & 23 & 145.8 & $\mathrm{NE}$ & 32.2 & 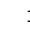 & 147 & NE & 33 & 1.08 & 0.2 & $\mathrm{~N}$ & 19.6 & 275 \\
\hline 3.981 & 54.82 & 1 & 147 & $\mathrm{NE}$ & 33 & 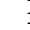 & 145 & $\mathrm{NE}$ & 23 & 10.04 & 150.8 & NW & 2.4 & 61 \\
\hline 3.981 & 58.8 & 1 & 145 & $\mathrm{NE}$ & 23 & 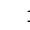 & 158 & $\mathrm{E}$ & 22 & 5.06 & \begin{tabular}{|l|}
73.7 \\
\end{tabular} & $E$ & 21.9 & 342 \\
\hline 3.981 & 62.78 & 1 & 158 & $\mathrm{E}$ & 22 & 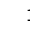 & 152 & $\mathrm{NE}$ & 22 & 2.25 & 65 & $\mathrm{NE}$ & 22 & 155 \\
\hline 3.981 & 66.76 & 1 & 152 & $\mathrm{NE}$ & 22 & 2 & 149.6 & $\mathrm{NE}$ & 14.4 & 7.61 & 156.2 & NW & 1.7 & 66 \\
\hline 3.981 & 70.74 & 2 & 149.6 & $\mathrm{NE}$ & 14.4 & 2 & 160.7 & $\mathrm{E}$ & 15.1 & 2.89 & 52 & $\mathrm{NE}$ & 14.3 & 321 \\
\hline 5.012 & 4.33 & 6 & 164 & E & 26.5 & 1 & 167.1 & $\mathrm{E}$ & 30.1 & 3.81 & 5.2 & $\mathrm{~N}$ & 10.2 & 278 \\
\hline 5.012 & 9.34 & 15 & 167.1 & $\mathrm{E}$ & 30.1 & 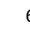 & 163.5 & $\mathrm{E}$ & 32.9 & 3.44 & 136 & SE & 16.6 & 222 \\
\hline 5.012 & 14.35 & 6 & 163.5 & E & 32.9 & 5 & 163.2 & E & 26.4 & 6.59 & 164.3 & $\mathrm{~N}$ & 0.6 & 75 \\
\hline 5.012 & 19.36 & 5 & 163.2 & E & 26.4 & 1 & 162.2 & E & 19.7 & 6.63 & 165.9 & $\mathrm{~N}$ & 1.3 & 76 \\
\hline 5.012 & 24.38 & 14 & 162.2 & E & 19.7 & 1 & 159.7 & E & 26.1 & 6.45 & 152.9 & SE & 3.3 & 242 \\
\hline 5.012 & 29.39 & 18 & 159.7 & E & 26.1 & 2 & 155.6 & $\mathrm{NE}$ & 26.4 & 1.81 & 79.5 & $\mathrm{E}$ & 25.8 & 167 \\
\hline 5.012 & 34.4 & 2 & 155.6 & $\mathrm{NE}$ & 26.4 & 1 & 145.8 & NE & 24 & 4.84 & 28.3 & $\mathrm{NE}$ & 21.6 & 121 \\
\hline 5.012 & 39.41 & 17 & 145.8 & $\mathrm{NE}$ & 24 & 1 & 153.7 & NE & 17.4 & 7.15 & 128.1 & SE & 7.7 & 37 \\
\hline 5.012 & 44.42 & 11 & 153.7 & $\mathrm{NE}$ & 17.4 & 2 & 147.7 & NE & 31.9 & 14.65 & 141.6 & SE & 3.8 & 231 \\
\hline 5.012 & 49.43 & 20 & 147.7 & $\mathrm{NE}$ & 31.9 & 5 & 146.6 & $\mathrm{NE}$ & 32 & 0.58 & 67.9 & $E$ & 31.5 & 157 \\
\hline 5.012 & 54.45 & 5 & 146.6 & $\mathrm{NE}$ & 32 & 2 & 151.4 & $\mathrm{NE}$ & 22.4 & 9.84 & 137.5 & SE & 5.6 & 46 \\
\hline 5.012 & 69.48 & 1 & 152 & $\mathrm{NE}$ & 22 & 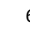 & 157 & NE & 14.8 & 7.39 & 142.7 & SE & 3.7 & 52 \\
\hline 6.31 & 5.36 & 10 & 166.6 & $\mathrm{E}$ & 27.6 & 1 & 165 & $\mathrm{E}$ & 30 & 2.46 & 150.2 & SE & 8.4 & 238 \\
\hline 6.31 & 11.67 & 14 & 165 & E & 30 & 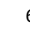 & 166.6 & $\mathrm{E}$ & 30.7 & 1.11 & 26.7 & $\mathrm{NE}$ & 21 & 305 \\
\hline 6.31 & 17.98 & 6 & 166.6 & E & 30.7 & 1 & 162.6 & E & 21.8 & 9.07 & 174.7 & $\mathrm{~N}$ & 4.8 & 86 \\
\hline 6.31 & 24.29 & 15 & 162.6 & $\mathrm{E}$ & 21.8 & 1 & 158.8 & $\mathrm{E}$ & 25.3 & 3.75 & 139 & SE & 9.1 & 227 \\
\hline 6.31 & 30.6 & 19 & 158.8 & $\mathrm{E}$ & 25.3 & 1 & 148.4 & NE & 25.7 & 4.49 & 69.3 & $E$ & 25.3 & 159 \\
\hline 6.31 & 36.91 & 15 & 148.4 & $\mathrm{NE}$ & 25.7 & 1 & 147.4 & $\mathrm{NE}$ & 16.7 & 9.03 & 150.1 & NW & 0.8 & 60 \\
\hline 6.31 & 43.21 & 13 & 147.4 & $\mathrm{NE}$ & 16.7 & 2 & 148.4 & NE & 31 & 14.37 & 149.4 & NW & 0.6 & 239 \\
\hline 6.31 & 49.52 & 22 & 148.4 & $\mathrm{NE}$ & 31 & 5 & 146.6 & $\mathrm{NE}$ & 32 & 1.35 & 105.9 & $E$ & 22.1 & 194 \\
\hline 6.31 & 55.83 & 5 & 146.6 & $\mathrm{NE}$ & 32 & 2 & 151.4 & NE & 22.4 & 9.84 & 137.5 & SE & 5.6 & 46 \\
\hline 6.31 & 62.14 & 2 & 151.4 & NE & 22.4 & 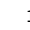 & 152 & NE & 22 & 0.44 & 120.6 & SE & 11.9 & 32 \\
\hline 6.31 & 68.45 & 1 & 152 & $\mathrm{NE}$ & 22 & 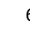 & 157 & $\mathrm{NE}$ & 14.8 & 7.39 & 142.7 & SE & 3.7 & 52 \\
\hline 7.943 & 5.13 & 10 & 166.6 & E & 27.6 & 1 & 165 & $\mathrm{E}$ & 30.4 & 2.86 & 152.4 & SE & 7.3 & 240 \\
\hline 7.943 & 13.08 & 15 & 165 & E & 30.4 & 7 & 164 & E & 29.7 & 0.85 & 16.1 & $\mathrm{~N}$ & 16.8 & 110 \\
\hline 7.943 & 21.02 & 7 & 164 & E & 29.7 & 3 & 160.6 & $\mathrm{E}$ & 23.4 & 6.47 & 174.5 & $\mathrm{~N}$ & 5.9 & 86 \\
\hline 7.943 & 28.96 & 32 & 160.6 & $\mathrm{E}$ & 23.4 & 1 & 148.4 & NE & 25.7 & 5.55 & 91.1 & $\mathrm{E}$ & 22.1 & 179 \\
\hline 7.943 & 36.91 & 15 & 148.4 & $\mathrm{NE}$ & 25.7 & 1 & 149.5 & NE & 17.6 & 8.11 & 146.4 & SE & 1 & 56 \\
\hline 7.943 & 44.85 & 15 & 149.5 & $\mathrm{NE}$ & 17.6 & 2 & 147.4 & $\mathrm{NE}$ & 31.9 & 14.33 & 145.3 & SE & 1.3 & 235 \\
\hline 7.943 & 52.79 & 25 & 147.4 & $\mathrm{NE}$ & 31.9 & 2 & 151.4 & $\mathrm{NE}$ & 22.4 & 9.69 & 139.8 & SE & 4.7 & 48 \\
\hline 7.943 & 60.73 & 2 & 151.4 & $\mathrm{NE}$ & 22.4 & . & 152 & NE & 22 & 0.44 & 120.6 & SE & 11.9 & 32 \\
\hline 7.943 & 68.68 & 1 & 152 & $\mathrm{NE}$ & 22 & 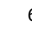 & 157 & $\mathrm{NE}$ & 14.8 & 7.39 & 142.7 & SE & 3.7 & 52 \\
\hline 10 & 6.91 & 17 & 166.2 & E & 29 & 1 & 165.7 & E & 30.5 & 1.51 & 157.9 & $\mathrm{~s}$ & 4.5 & 247 \\
\hline
\end{tabular}




\begin{tabular}{|c|c|c|c|c|c|c|c|c|c|c|c|c|c|c|}
\hline \multicolumn{15}{|l|}{ Section II } \\
\hline & elevation between & number & average & & & $\begin{array}{l}\text { upper window } \\
\text { number }\end{array}$ & aver & & & & & & & \\
\hline $\begin{array}{l}\text { window size } \\
(\mathrm{m})\end{array}$ & $\begin{array}{c}\text { two consecutive windows } \\
(\mathrm{m})\end{array}$ & of measures & orientation & & & of measures & orientation & & & |tilt angle & tilt axis & & & |tilt way \\
\hline 0.02 & 45.43 & 1 & 115 & $\mathrm{NE}$ & 26 & 1 & 141 & $\mathrm{NE}$ & 33 & 14.45 & 6.4 & $\mathrm{~N}$ & 24.8 & 278 \\
\hline 0.025 & 44.78 & 1 & 145 & $\mathrm{NE}$ & 37 & 1 & 172 & E & 26 & 17.66 & 110.2 & E & 23.3 & 14 \\
\hline 0.025 & 45.44 & 1 & 115 & $\mathrm{NE}$ & 26 & 1 & 141 & $\mathrm{NE}$ & 33 & 14.45 & 6.4 & $\mathrm{~N}$ & 24.8 & 278 \\
\hline 0.032 & 11.76 & 1 & 8 & $\mathrm{~s}$ & 24 & 1 & 170 & E & 35 & 14.02 & 143.6 & SE & 17.3 & 231 \\
\hline 0.032 & 44.96 & 1 & 155 & $\mathrm{NE}$ & 28 & 1 & 156 & $\mathrm{NE}$ & 34 & 6.02 & 159.7 & $\mathrm{~N}$ & 2.5 & 250 \\
\hline 0.032 & 45.43 & 1 & 115 & $\mathrm{NE}$ & 26 & 1 & 141 & $\mathrm{NE}$ & 33 & 14.45 & 6.4 & $\mathrm{~N}$ & 24.8 & 278 \\
\hline 0.04 & 11.77 & 1 & 8 & $\mathrm{~s}$ & 24 & 1 & 170 & $\mathrm{E}$ & 35 & 14.02 & 143.6 & $\mathrm{SE}$ & 17.3 & 231 \\
\hline 0.04 & 44.49 & 1 & 170 & E & 32 & 1 & 167 & E & 36 & 4.34 & 149.3 & SE & 12.5 & 236 \\
\hline 0.04 & 44.61 & 1 & 160 & E & 37 & 1 & 145 & $\mathrm{NE}$ & 34 & 9.19 & 39.7 & $\mathrm{NE}$ & 33 & 135 \\
\hline 0.04 & 44.73 & 1 & 152 & $\mathrm{NE}$ & 33 & 1 & 145 & $\mathrm{NE}$ & 37 & 5.66 & 109 & $\mathrm{E}$ & 23.9 & 194 \\
\hline 0.04 & 44.77 & 1 & 145 & $\mathrm{NE}$ & 37 & 1 & 172 & $\mathrm{E}$ & 26 & 17.66 & 110.2 & $\mathrm{E}$ & 23.3 & 14 \\
\hline 0.04 & 44.89 & 1 & 148 & $\mathrm{NE}$ & 32 & 1 & 155 & $\mathrm{NE}$ & 28 & 5.31 & 114.3 & $\mathrm{SE}$ & 19.1 & 20 \\
\hline 0.04 & 45.09 & 1 & 175 & E & 16 & 1 & 151 & $\mathrm{NE}$ & 23 & 10.51 & 115.3 & $\mathrm{SE}$ & 13.9 & 204 \\
\hline 0.04 & 45.21 & 1 & 158 & $\mathrm{E}$ & 33 & 1 & 159 & E & 29 & 4.03 & 152.2 & SE & 3.8 & 61 \\
\hline 0.04 & 45.33 & 1 & 165 & $\mathrm{E}$ & 30 & 1 & 48 & NW & 8 & 34.31 & \begin{tabular}{|l}
$\mid$ \\
\end{tabular} & $\mathrm{N}$ & 6.3 & 88 \\
\hline 0.04 & 45.45 & 1 & 115 & $\mathrm{NE}$ & 26 & 1 & 141 & $\mathrm{NE}$ & 33 & 14.45 & 6.4 & $\mathrm{~N}$ & 24.8 & 278 \\
\hline 0.04 & 45.49 & 1 & 141 & $\mathrm{NE}$ & 33 & 1 & 152 & $\mathrm{NE}$ & 53 & 21.3 & 162.2 & $\mathrm{~N}$ & 13.2 & 256 \\
\hline 0.04 & 45.61 & 1 & 140 & $\mathrm{NE}$ & 52 & 1 & 167 & $\mathrm{E}$ & 30 & 27.84 & 121.1 & SE & 22.5 & 21 \\
\hline 0.05 & 11.7 & 1 & 8 & $\mathrm{~S}$ & 28 & 1 & 8 & S & 24 & 4 & 8 & $\mathrm{~s}$ & 0 & 98 \\
\hline 0.05 & 11.75 & 1 & 8 & $\mathrm{~s}$ & 24 & 1 & 170 & $\mathrm{E}$ & 35 & 14.02 & 143.6 & $\mathrm{SE}$ & 17.3 & 231 \\
\hline 0.05 & 32 & 1 & 165 & $\mathrm{E}$ & 25 & 1 & 10 & $\mathrm{~s}$ & 15 & 12.95 & 138.1 & $\mathrm{SE}$ & 11.9 & 46 \\
\hline 0.05 & 32.45 & 1 & 146 & $\mathrm{NE}$ & 23 & 1 & 164 & $\mathrm{E}$ & 27 & 8.55 & 35.1 & $\mathrm{NE}$ & 21.6 & 307 \\
\hline 0.05 & 32.6 & 1 & 159 & $\mathrm{E}$ & 27 & 1 & 175 & E & 25 & 7.27 & 94.5 & $\mathrm{E}$ & 24.7 & 2 \\
\hline 0.05 & 44.48 & 1 & 170 & $\mathrm{E}$ & 32 & 1 & 167 & $\mathrm{E}$ & 36 & 4.34 & 149.3 & $\mathrm{SE}$ & 12.5 & 236 \\
\hline 0.05 & 44.63 & 1 & 160 & $\mathrm{E}$ & 37 & 1 & 145 & $\mathrm{NE}$ & 34 & 9.19 & 39.7 & $\mathrm{NE}$ & 33 & 135 \\
\hline 0.05 & 44.68 & 1 & 145 & $\mathrm{NE}$ & 34 & 1 & 152 & $\mathrm{NE}$ & 33 & 3.99 & 75.7 & $\mathrm{E}$ & 32.2 & 342 \\
\hline 0.05 & 44.73 & 1 & 152 & $\mathrm{NE}$ & 33 & 1 & 145 & $\mathrm{NE}$ & 37 & 5.66 & 109 & $\mathrm{E}$ & 23.9 & 194 \\
\hline 0.05 & 44.78 & 1 & 145 & $\mathrm{NE}$ & 37 & 1 & 172 & $\mathrm{E}$ & 26 & 17.66 & 110.2 & E & 23.3 & 14 \\
\hline 0.05 & 44.83 & 1 & 172 & $\mathrm{E}$ & 26 & 1 & 148 & $\mathrm{NE}$ & 32 & 12.98 & 100.1 & $\mathrm{E}$ & 24.9 & 188 \\
\hline 0.05 & 44.88 & 1 & 148 & $\mathrm{NE}$ & 32 & 1 & 155 & $\mathrm{NE}$ & 28 & 5.31 & 114.3 & $\mathrm{SE}$ & 19.1 & 20 \\
\hline 0.05 & 45.03 & 1 & 156 & $\mathrm{NE}$ & 34 & 1 & 175 & E & 16 & 19.5 & 143 & SE & 8.6 & 50 \\
\hline 0.05 & 45.08 & 1 & 175 & $\mathrm{E}$ & 16 & 1 & 151 & $\mathrm{NE}$ & 23 & 10.51 & 115.3 & $\mathrm{SE}$ & 13.9 & 204 \\
\hline 0.05 & 45.13 & 1 & 151 & $\mathrm{NE}$ & 23 & 1 & 158 & E & 33 & 10.51 & 170.8 & $\mathrm{~N}$ & 8.2 & 262 \\
\hline 0.05 & 45.28 & 1 & 159 & $\mathrm{E}$ & 29 & 1 & 165 & $\mathrm{E}$ & 30 & 3.12 & 50.8 & $\mathrm{NE}$ & 27.8 & 323 \\
\hline 0.05 & 45.33 & 1 & 165 & $\mathrm{E}$ & 30 & 1 & 48 & NW & 8 & 34.31 & 176.1 & $\mathrm{~N}$ & 6.3 & 88 \\
\hline 0.05 & 45.38 & 1 & 48 & NW & 8 & 1 & 115 & $\mathrm{NE}$ & 26 & 23.95 & 131.6 & NW & 8 & 222 \\
\hline 0.05 & 45.43 & 1 & 115 & $\mathrm{NE}$ & 26 & 1 & 141 & $\mathrm{NE}$ & 33 & 14.45 & 6.4 & $\mathrm{~N}$ & 24.8 & 278 \\
\hline 0.05 & 45.48 & 1 & 141 & $\mathrm{NE}$ & 33 & 1 & 152 & $\mathrm{NE}$ & 53 & 21.3 & 162.2 & $\mathrm{~N}$ & 13.2 & 256 \\
\hline 0.05 & 45.53 & 1 & 152 & $\mathrm{NE}$ & 53 & 1 & 140 & $\mathrm{NE}$ & 52 & 9.57 & 46.2 & $\mathrm{NE}$ & 51.9 & 142 \\
\hline 0.05 & 45.68 & 1 & 167 & $\mathrm{E}$ & 30 & 1 & 158 & $\mathrm{E}$ & 28 & 4.79 & 44.9 & $\mathrm{NE}$ & 26.1 & 138 \\
\hline 0.063 & 8.37 & 1 & 132 & $\mathrm{NE}$ & 41 & 1 & 137 & $\mathrm{NE}$ & 28 & 13.3 & 124.2 & $\mathrm{SE}$ & 6.7 & 32 \\
\hline 0.063 & 11.72 & 1 & 8 & $\mathrm{~S}$ & 28 & 2 & 177.5 & $\mathrm{E}$ & 29.2 & 5.18 & 107.9 & $E$ & 27.6 & 197 \\
\hline 0.063 & 32.1 & 1 & 10 & $\mathrm{~S}$ & 15 & 1 & 155 & $\mathrm{NE}$ & 26 & 16.03 & 125.2 & $\mathrm{SE}$ & 13.6 & 214 \\
\hline 0.063 & 32.16 & 1 & 155 & $\mathrm{NE}$ & 26 & 1 & 141 & $\mathrm{NE}$ & 24 & 6.23 & 37.6 & $\mathrm{NE}$ & 23.4 & 130 \\
\hline 0.063 & 32.35 & 1 & 142 & $\mathrm{NE}$ & 24 & 1 & 146 & $\mathrm{NE}$ & 23 & 1.88 & 88.3 & $\mathrm{E}$ & 19.7 & 356 \\
\hline 0.063 & 32.54 & 1 & 164 & $\mathrm{E}$ & 27 & 1 & 159 & $\mathrm{E}$ & 27 & 2.27 & 71.5 & $\mathrm{E}$ & 27 & 162 \\
\hline 0.063 & 32.6 & 1 & 159 & E & 27 & 1 & 175 & E & 25 & 7.27 & 94.5 & $E$ & 24.7 & 2 \\
\hline 0.063 & 32.92 & 1 & 171 & E & 25 & 1 & 179 & E & 30 & 6.21 & 28.3 & $\mathrm{NE}$ & 15.8 & 301 \\
\hline 0.063 & 44.59 & 2 & 163.5 & $\mathrm{E}$ & 36.4 & 1 & 145 & $\mathrm{NE}$ & 34 & 10.89 & 48.6 & $\mathrm{NE}$ & 33.8 & 143 \\
\hline 0.063 & 44.65 & 1 & 145 & $\mathrm{NE}$ & 34 & 1 & 152 & $\mathrm{NE}$ & 33 & 3.99 & 75.7 & $\mathrm{E}$ & 32.2 & 342 \\
\hline 0.063 & 44.72 & 1 & 152 & $\mathrm{NE}$ & 33 & 1 & 145 & $\mathrm{NE}$ & 37 & 5.66 & 109 & $\mathrm{E}$ & 23.9 & 194 \\
\hline 0.063 & 44.78 & 1 & 145 & $\mathrm{NE}$ & 37 & 1 & 172 & E & 26 & 17.66 & 110.2 & E & 23.3 & 14 \\
\hline 0.063 & 44.84 & 1 & 172 & $E$ & 26 & 1 & 148 & $\mathrm{NE}$ & 32 & 12.98 & 100.1 & $\mathrm{E}$ & 24.9 & 188 \\
\hline 0.063 & 44.91 & 1 & 148 & $\mathrm{NE}$ & 32 & 1 & 155 & $\mathrm{NE}$ & 28 & 5.31 & 114.3 & $\mathrm{SE}$ & 19.1 & 20 \\
\hline 0.063 & 44.97 & 1 & 155 & $\mathrm{NE}$ & 28 & 1 & 156 & $\mathrm{NE}$ & 34 & 6.02 & 159.7 & $\mathrm{~N}$ & 2.5 & 250 \\
\hline 0.063 & 45.03 & 1 & 156 & $\mathrm{NE}$ & 34 & 1 & 175 & $\mathrm{E}$ & 16 & 19.5 & 143 & SE & 8.6 & 50 \\
\hline 0.063 & 45.1 & 1 & 175 & $\mathrm{E}$ & 16 & 1 & 151 & $\mathrm{NE}$ & 23 & 10.51 & 115.3 & SE & 13.9 & 204 \\
\hline 0.063 & 45.16 & 1 & 151 & $\mathrm{NE}$ & 23 & 1 & 158 & E & 33 & 10.51 & 170.8 & $\mathrm{~N}$ & 8.2 & 262 \\
\hline 0.063 & 45.22 & 1 & 158 & $E$ & 33 & 1 & 159 & $\mathrm{E}$ & 29 & 4.03 & 152.2 & $\mathrm{SE}$ & 3.8 & 61 \\
\hline 0.063 & 45.29 & 1 & 159 & E & 29 & 1 & 165 & $E$ & 30 & 3.12 & 50.8 & $\mathrm{NE}$ & 27.8 & 323 \\
\hline 0.063 & 45.35 & 1 & 165 & E & 30 & 1 & 48 & NW & 8 & 34.31 & 176.1 & $\mathrm{~N}$ & 6.3 & 88 \\
\hline 0.063 & 45.41 & 1 & 48 & NW & 8 & 2 & 129.4 & $\mathrm{NE}$ & 28.9 & 28.7 & 144.1 & NW & 8 & 234 \\
\hline 0.063 & 45.47 & 2 & 129.4 & $\mathrm{NE}$ & 28.9 & 1 & 152 & $\mathrm{NE}$ & 53 & 27.98 & 166.5 & $\mathrm{~N}$ & 18.4 & 260 \\
\hline 0.063 & 45.54 & 1 & 152 & $\mathrm{NE}$ & 53 & 1 & 140 & $\mathrm{NE}$ & 52 & 9.57 & 46.2 & $\mathrm{NE}$ & 51.9 & 142 \\
\hline 0.063 & 45.6 & 1 & 140 & $\mathrm{NE}$ & 52 & 1 & 167 & $\mathrm{E}$ & 30 & 27.84 & 121.1 & $\mathrm{SE}$ & 22.5 & 21 \\
\hline 0.063 & 45.66 & 1 & 167 & $\mathrm{E}$ & 30 & 1 & 158 & $\mathrm{E}$ & 28 & 4.79 & 44.9 & $\mathrm{NE}$ & 26.1 & 138 \\
\hline 0.079 & 8 & 1 & 142 & $\mathrm{NE}$ & 30 & 1 & 162 & E & 41 & 15.88 & 13.1 & $\mathrm{~N}$ & 24.2 & 287 \\
\hline 0.079 & 8.4 & 1 & 132 & $\mathrm{NE}$ & 41 & 1 & 137 & $\mathrm{NE}$ & 28 & 13.3 & 124.2 & SE & 6.7 & 32 \\
\hline 0.079 & 11.73 & 2 & 8 & $\mathrm{E}$ & 26 & 1 & 170 & $\mathrm{E}$ & 35 & 12.74 & 137.5 & $\mathrm{SE}$ & 20.6 & 225 \\
\hline 0.079 & 31.99 & 1 & 165 & $\mathrm{E}$ & 25 & 1 & 10 & $\mathrm{~S}$ & 15 & 12.95 & 138.1 & $\mathrm{SE}$ & 11.9 & 46 \\
\hline 0.079 & 32.07 & 1 & 10 & $\mathrm{~S}$ & 15 & 1 & 155 & $\mathrm{NE}$ & 26 & 16.03 & 125.2 & SE & 13.6 & 214 \\
\hline 0.079 & 32.15 & 1 & 155 & $\mathrm{NE}$ & 26 & 1 & 141 & $\mathrm{NE}$ & 24 & 6.23 & 37.6 & $\mathrm{NE}$ & 23.4 & 130 \\
\hline 0.079 & 32.39 & 1 & 142 & $\mathrm{NE}$ & 24 & 1 & 146 & $\mathrm{NE}$ & 23 & 1.88 & 88.3 & $\mathrm{E}$ & 19.7 & 356 \\
\hline 0.079 & 32.47 & 1 & 146 & $\mathrm{NE}$ & 23 & 1 & 164 & $\mathrm{E}$ & 27 & 8.55 & 35.1 & $\mathrm{NE}$ & 21.6 & 307 \\
\hline
\end{tabular}




\begin{tabular}{|c|c|c|c|c|c|c|c|c|c|c|c|c|c|c|}
\hline 0.1 & 32.25 & 1 & 141 & $\mathrm{NE}$ & 24|| & 1 & 142 & $\mathrm{NE}$ & 24 & 0.41 & 51.5 & $\mathrm{NE}$ & 24 & 321 \\
\hline 0.1 & 32.35 & 1 & 142 & $\mathrm{NE}$ & 24 & 1 & 146 & $\mathrm{NE}$ & 23 & 1.88 & 88.3 & E & 19.7 & 356 \\
\hline 0.1 & 32.45 & 1 & 146 & $\mathrm{NE}$ & 23 & 1 & 164 & $\mathrm{E}$ & 27 & 8.55 & 35.1 & $\mathrm{NE}$ & 21.6 & 307 \\
\hline 0.1 & 32.55 & 1 & 164 & $E$ & 27 & 2 & 166.7 & $E$ & 25.8 & 1.71 & 123.8 & $\mathrm{SE}$ & 18.2 & 30 \\
\hline 0.1 & 32.65 & 2 & 166.7 & $\mathrm{E}$ & 25.8 & 1 & 155 & NE & 23 & 5.57 & 38.7 & $\mathrm{NE}$ & 20.8 & 131 \\
\hline 0.1 & 32.95 & 1 & 171 & $\mathrm{E}$ & 25 & 1 & 179 & $E$ & 30 & 6.21 & 28.3 & $\mathrm{NE}$ & 15.8 & 301 \\
\hline 0.1 & 34.25 & 1 & 158 & $\mathrm{E}$ & 33 & 1 & 55 & NW & 24 & 44.25 & 8.1 & $\mathrm{~N}$ & 18 & 103 \\
\hline 0.1 & 44.55 & 2 & 168.4 & $E$ & 34 & 2 & 152.8 & $\mathrm{NE}$ & 35.3 & 8.96 & 80.4 & E & 34 & 170 \\
\hline 0.1 & 44.65 & 2 & 152.8 & $\mathrm{NE}$ & 35.3 & 1 & 152 & $\mathrm{NE}$ & 33 & 2.31 & 161.4 & $\mathrm{~N}$ & 6.1 & 74 \\
\hline 0.1 & 44.75 & 1 & 152 & $\mathrm{NE}$ & 33 & 2 & 156.3 & $\mathrm{NE}$ & 30.8 & 3.17 & 112.5 & SE & 22.4 & 18 \\
\hline 0.1 & 44.85 & 2 & 156.3 & $\mathrm{NE}$ & 30.8 & 2 & 151.3 & $\mathrm{NE}$ & 30 & 2.69 & 42.7 & $\mathrm{NE}$ & 28.6 & 137 \\
\hline 0.1 & 44.95 & 2 & 151.3 & $\mathrm{NE}$ & 30 & 2 & 162.2 & $\mathrm{E}$ & 24.7 & 7.23 & 116.1 & SE & 18.4 & 23 \\
\hline 0.1 & 45.05 & 2 & 162.2 & $E$ & 24.7 & 1 & 151 & $\mathrm{NE}$ & 23 & 4.86 & 44.1 & $\mathrm{NE}$ & 22.1 & 137 \\
\hline 0.1 & 45.15 & 1 & 151 & $\mathrm{NE}$ & 23 & 2 & 158.5 & $E$ & 31 & 8.67 & 175.5 & $\mathrm{~N}$ & 10 & 267 \\
\hline 0.1 & 45.25 & 2 & 158.5 & $\mathrm{E}$ & 31 & 1 & 165 & $\mathrm{E}$ & 30 & 3.46 & 91 & E & 29 & 358 \\
\hline 0.1 & 45.35 & 1 & 165 & $\mathrm{E}$ & 30 & 3 & 121.5 & $\mathrm{NE}$ & 20.1 & 20.29 & 23.9 & $\mathrm{NE}$ & 19.9 & 118 \\
\hline 0.1 & 45.45 & 3 & 121.5 & $\mathrm{NE}$ & 20.1 & 1 & 152 & $\mathrm{NE}$ & 53 & 36.71 & 162.4 & $\mathrm{~N}$ & 13.5 & 254 \\
\hline 0.1 & 45.55 & 1 & 152 & $\mathrm{NE}$ & 53 & 2 & 150.4 & $\mathrm{NE}$ & 40.2 & 12.8 & 154.8 & NW & 3.7 & 67 \\
\hline 0.1 & 45.65 & 2 & 150.4 & $\mathrm{NE}$ & 40.2 & 1 & 158 & $\mathrm{E}$ & 28 & 12.94 & 138.1 & SE & 10.3 & 44 \\
\hline 0.126 & 8 & 1 & 142 & $\mathrm{NE}$ & 30 & 1 & 162 & $\mathrm{E}$ & 41 & 15.88 & 13.1 & $\mathrm{~N}$ & 24.2 & 287 \\
\hline 0.126 & 8.13 & 1 & 162 & $E$ & 41 & 1 & 166 & $\mathrm{E}$ & 22 & 19.11 & 158.5 & S & 3 & 67 \\
\hline 0.126 & 8.25 & 1 & 166 & $E$ & 22 & 1 & 132 & $\mathrm{NE}$ & 41 & 25.38 & 109.1 & $\mathrm{E}$ & 18.7 & 197 \\
\hline 0.126 & 8.38 & 1 & 132 & $\mathrm{NE}$ & 41 & 1 & 137 & $\mathrm{NE}$ & 28 & 13.3 & 124.2 & SE & 6.7 & 32 \\
\hline 0.126 & 8.5 & 1 & 137 & $\mathrm{NE}$ & 28 & 1 & 134 & $\mathrm{NE}$ & 35 & 7.17 & 124.7 & SE & 6.5 & 213 \\
\hline 0.126 & 11.78 & 2 & 8 & $\mathrm{E}$ & 26 & 1 & 170 & $\mathrm{E}$ & 35 & 12.74 & 137.5 & SE & 20.6 & 225 \\
\hline 0.126 & 32.05 & 1 & 165 & $\mathrm{E}$ & 25 & 2 & 167.9 & $\mathrm{E}$ & 19.7 & 5.42 & 155.7 & SE & 4.3 & 65 \\
\hline 0.126 & 32.17 & 2 & 167.9 & E & 19.7 & 1 & 141 & $\mathrm{NE}$ & 24 & 10.77 & 89 & $E$ & 19.3 & 178 \\
\hline 0.126 & 32.3 & 1 & 141 & $\mathrm{NE}$ & 24 & 2 & 144 & $\mathrm{NE}$ & 23.5 & 1.3 & 77.7 & $\mathrm{E}$ & 21.7 & 345 \\
\hline 0.126 & 32.42 & 2 & 144 & $\mathrm{NE}$ & 23.5 & 1 & 164 & $\mathrm{E}$ & 27 & 9.19 & 39.8 & $\mathrm{NE}$ & 22.8 & 311 \\
\hline 0.126 & 32.55 & 1 & 164 & $\mathrm{E}$ & 27 & 2 & 166.7 & $\mathrm{E}$ & 25.8 & 1.71 & 123.8 & SE & 18.2 & 30 \\
\hline 0.126 & 32.67 & 2 & 166.7 & $\mathrm{E}$ & 25.8 & 1 & 155 & $\mathrm{NE}$ & 23 & 5.57 & 38.7 & $\mathrm{NE}$ & 20.8 & 131 \\
\hline 0.126 & 32.8 & 1 & 155 & $\mathrm{NE}$ & 23 & 1 & 171 & $E$ & 25 & 6.79 & 54.5 & $\mathrm{NE}$ & 22.7 & 325 \\
\hline 0.126 & 32.93 & 1 & 171 & $\mathrm{E}$ & 25 & 1 & 179 & $\mathrm{E}$ & 30 & 6.21 & 28.3 & $\mathrm{NE}$ & 15.8 & 301 \\
\hline 0.126 & 34.31 & 1 & 158 & $\mathrm{E}$ & 33 & 1 & 55 & NW & 24 & 44.25 & 8.1 & $\mathrm{~N}$ & 18 & 103 \\
\hline 0.126 & 44.51 & 1 & 170 & $\mathrm{E}$ & 32 & 2 & 163.5 & $\mathrm{E}$ & 36.4 & 5.77 & 132.3 & SE & 20.9 & 218 \\
\hline 0.126 & 44.63 & 2 & 163.5 & $\mathrm{E}$ & 36.4 & 2 & 148.5 & $\mathrm{NE}$ & 33.5 & 9.08 & 43 & $\mathrm{NE}$ & 32.5 & 139 \\
\hline 0.126 & 44.76 & 2 & 148.5 & $\mathrm{NE}$ & 33.5 & 3 & 153.4 & $\mathrm{NE}$ & 31.2 & 3.5 & 106.4 & $\mathrm{E}$ & 23.9 & 12 \\
\hline 0.126 & 44.89 & 3 & 153.4 & $\mathrm{NE}$ & 31.2 & 2 & 155.5 & $\mathrm{NE}$ & 31 & 1.11 & 74.5 & $\mathrm{E}$ & 30.7 & 345 \\
\hline 0.126 & 45.01 & 2 & 155.5 & $\mathrm{NE}$ & 31 & 2 & 160.9 & $\mathrm{E}$ & 19.1 & 12.09 & 148.3 & SE & 4.3 & 57 \\
\hline 0.126 & 45.14 & 2 & 160.9 & $\mathrm{E}$ & 19.1 & 2 & 158.5 & $\mathrm{E}$ & 31 & 11.92 & 155.2 & $\mathrm{SE}$ & 2 & 245 \\
\hline 0.126 & 45.26 & 2 & 158.5 & $\mathrm{E}$ & 31 & 2 & 149.2 & $\mathrm{NE}$ & 13.7 & 17.56 & 164.8 & $\mathrm{~N}$ & 3.8 & 76 \\
\hline 0.126 & 45.39 & 2 & 149.2 & $\mathrm{NE}$ & 13.7 & 2 & 129.4 & $\mathrm{NE}$ & 28.9 & 16.54 & 115 & SE & 7.8 & 204 \\
\hline 0.126 & 45.52 & 2 & 129.4 & $\mathrm{NE}$ & 28.9 & 3 & 150.9 & $\mathrm{NE}$ & 44.7 & 20.25 & 173.8 & $\mathrm{~N}$ & 21.1 & 268 \\
\hline 0.126 & 45.64 & 3 & 150.9 & $\mathrm{NE}$ & 44.7 & 1 & 158 & $E$ & 28 & 17.24 & 142.9 & SE & 7.9 & 50 \\
\hline 0.158 & 8.04 & 1 & 142 & $\mathrm{NE}$ & 30 & 2 & 163.5 & $\mathrm{E}$ & 31.5 & 11.02 & 53.9 & $\mathrm{NE}$ & 30 & 324 \\
\hline 0.158 & 8.19 & 2 & 163.5 & $\mathrm{E}$ & 31.5 & 1 & 132 & $\mathrm{NE}$ & 41 & 20.63 & 89.3 & $\mathrm{E}$ & 30.5 & 177 \\
\hline 0.158 & 8.35 & 1 & 132 & $\mathrm{NE}$ & 41 & 1 & 137 & $\mathrm{NE}$ & 28 & 13.3 & 124.2 & SE & 6.7 & 32 \\
\hline 0.158 & 8.51 & 1 & 137 & $\mathrm{NE}$ & 28 & 1 & 134 & $\mathrm{NE}$ & 35 & 7.17 & 124.7 & $\mathrm{SE}$ & 6.5 & 213 \\
\hline 0.158 & 11.52 & 1 & 172 & $\mathrm{E}$ & 29 & 1 & 8 & $\mathrm{~s}$ & 28 & 7.68 & 98.4 & $E$ & 28 & 6 \\
\hline 0.158 & 11.68 & 1 & 8 & S & 28 & 2 & 177.5 & $\mathrm{E}$ & 29.2 & 5.18 & 107.9 & E & 27.6 & 197 \\
\hline 0.158 & 32.13 & 2 & 174.4 & $\mathrm{E}$ & 19.6 & 2 & 148.3 & $\mathrm{NE}$ & 24.8 & 11.08 & 100.7 & E & 18.9 & 190 \\
\hline 0.158 & 32.29 & 2 & 148.3 & $\mathrm{NE}$ & 24.8 & 2 & 144 & $\mathrm{NE}$ & 23.5 & 2.22 & 16.1 & $\mathrm{~N}$ & 18.9 & 110 \\
\hline 0.158 & 32.44 & 2 & 144 & $\mathrm{NE}$ & 23.5 & 2 & 161.5 & $\mathrm{E}$ & 27 & 8.22 & 35.6 & $\mathrm{NE}$ & 22.4 & 307 \\
\hline 0.158 & 32.6 & 2 & 161.5 & $\mathrm{E}$ & 27 & 2 & 165.4 & $\mathrm{E}$ & 23.7 & 3.7 & 138.9 & $\mathrm{SE}$ & 11.1 & 46 \\
\hline 0.158 & 32.76 & 2 & 165.4 & $\mathrm{E}$ & 23.7 & 1 & 171 & $\mathrm{E}$ & 25 & 2.66 & 46 & $\mathrm{NE}$ & 20.9 & 319 \\
\hline 0.158 & 32.92 & 1 & 171 & $\mathrm{E}$ & 25 & 1 & 179 & $\mathrm{E}$ & 30 & 6.21 & 28.3 & $\mathrm{NE}$ & 15.8 & 301 \\
\hline 0.158 & 44.49 & 1 & 170 & $\mathrm{E}$ & 32 & 2 & 163.5 & $E$ & 36.4 & 5.77 & 132.3 & $\mathrm{SE}$ & 20.9 & 218 \\
\hline 0.158 & 44.65 & 2 & 163.5 & $\mathrm{E}$ & 36.4 & 4 & 152.2 & $\mathrm{NE}$ & 32.1 & 7.69 & 28.3 & $\mathrm{NE}$ & 27.5 & 125 \\
\hline 0.158 & 44.81 & 4 & 152.2 & $\mathrm{NE}$ & 32.1 & 2 & 151.3 & $\mathrm{NE}$ & 30 & 2.2 & 162 & $\mathrm{~N}$ & 6.1 & 74 \\
\hline 0.158 & 44.96 & 2 & 151.3 & $\mathrm{NE}$ & 30 & 3 & 158.6 & $\mathrm{E}$ & 24.1 & 6.76 & 128.2 & SE & 12.7 & 35 \\
\hline 0.158 & 45.12 & 3 & 158.6 & $E$ & 24.1 & 2 & 158.5 & $E$ & 31 & 6.95 & 158.1 & $S$ & 0.2 & 248 \\
\hline 0.158 & 45.28 & 2 & 158.5 & $\mathrm{E}$ & 31 & 3 & 132.1 & $\mathrm{NE}$ & 17.3 & 17.13 & 1.7 & $\mathrm{~N}$ & 13.3 & 95 \\
\hline 0.158 & 45.44 & 3 & 132.1 & $\mathrm{NE}$ & 17.3 & 3 & 145.3 & $\mathrm{NE}$ & 45.9 & 29.24 & 150.9 & NW & 5.7 & 242 \\
\hline 0.158 & 45.6 & 3 & 145.3 & $\mathrm{NE}$ & 45.9 & 2 & 162.6 & $\mathrm{E}$ & 28.9 & 19.82 & 127.2 & $\mathrm{SE}$ & 17.8 & 30 \\
\hline 0.2 & 2.1 & 1 & 180 & $\mathrm{E}$ & 32 & 1 & 165 & $E$ & 37 & 9.83 & 117.8 & SE & 28.9 & 204 \\
\hline 0.2 & 7.49 & 1 & 86 & $\mathrm{~N}$ & 33 & 1 & 125 & $\mathrm{NE}$ & 32 & 20.69 & 18.6 & $\mathrm{~N}$ & 30.9 & 285 \\
\hline 0.2 & 8.09 & 2 & 153.4 & $\mathrm{NE}$ & 35.1 & 1 & 166 & $\mathrm{E}$ & 22 & 14.35 & 137.4 & $\mathrm{SE}$ & 11 & 44 \\
\hline 0.2 & 8.29 & 1 & 166 & $\mathrm{E}$ & 22 & 2 & 134.1 & $\mathrm{NE}$ & 34.5 & 19.21 & 102.2 & $\mathrm{E}$ & 19.9 & 191 \\
\hline 0.2 & 8.49 & 2 & 134.1 & $\mathrm{NE}$ & 34.5 & 1 & 134 & $\mathrm{NE}$ & 35 & 0.53 & 129.7 & SE & 3 & 218 \\
\hline 0.2 & 11.48 & 1 & 172 & $\mathrm{E}$ & 29 & 1 & 8 & $\mathrm{~S}$ & 28 & 7.68 & 98.4 & $\mathrm{E}$ & 28 & 6 \\
\hline 0.2 & 11.68 & 1 & 8 & S & 28 & 2 & 177.5 & $\mathrm{E}$ & 29.2 & 5.18 & 107.9 & E & 27.6 & 197 \\
\hline 0.2 & 32.03 & 1 & 165 & $\mathrm{E}$ & 25 & 3 & 157.7 & $\mathrm{E}$ & 20.7 & 5.18 & 12.6 & $\mathrm{~N}$ & 12.2 & 105 \\
\hline 0.2 & 32.23 & 3 & 157.7 & $\mathrm{E}$ & 20.7 & 2 & 144 & $\mathrm{NE}$ & 23.5 & 5.85 & 91.2 & E & 19.1 & 180 \\
\hline 0.2 & 32.43 & 2 & 144 & $\mathrm{NE}$ & 23.5 & 2 & 161.5 & $E$ & 27 & 8.22 & 35.6 & $\mathrm{NE}$ & 22.4 & 307 \\
\hline 0.2 & 32.63 & 2 & 161.5 & $E$ & 27 & 2 & 165.4 & $\mathrm{E}$ & 23.7 & 3.7 & 138.9 & SE & 11.1 & 46 \\
\hline 0.2 & 32.83 & 2 & 165.4 & E & 23.7 & 2 & 175.3 & $\mathrm{E}$ & 27.4 & 5.7 & 36.2 & $\mathrm{NE}$ & 18.8 & 309 \\
\hline 0.2 & 34.03 & 1 & 143 & $\mathrm{NE}$ & 22 & 1 & 158 & $\mathrm{E}$ & 33 & 12.92 & 180 & $N$ & 13.7 & 272 \\
\hline 0.2 & 34.23 & 1 & 158 & $\mathrm{E}$ & 33 & 1 & 55 & NW & 24 & 44.25 & 8.1 & $\mathrm{~N}$ & 18 & 103 \\
\hline 0.2 & 44.6 & 3 & 165.5 & $\mathrm{E}$ & 34.9 & 4 & 152.2 & $\mathrm{NE}$ & 32.1 & 7.86 & 44.2 & $\mathrm{NE}$ & 30.8 & 139 \\
\hline
\end{tabular}




\begin{tabular}{|c|c|c|c|c|c|c|c|c|c|c|c|c|c|c|}
\hline 0.316 & 11.63 & 1 & 172 & $E$ & 29 & 3 & 0.9 & E & 28.7 & 4.31 & 91 & E & 28.7 & 360 \\
\hline 0.316 & 12.89 & 1 & 165 & $\mathrm{E}$ & 35 & 1 & 173 & $\mathrm{E}$ & 30 & 6.58 & 133 & SE & 20.4 & 38 \\
\hline 0.316 & 17.64 & 1 & 4 & $S$ & 18 & 1 & 176 & E & 13 & 5.43 & 22.5 & $\mathrm{~N}$ & 5.9 & 113 \\
\hline 0.316 & 32.18 & 3 & 166.7 & E & 21.5 & 4 & 148.7 & $\mathrm{NE}$ & 24.2 & 7.48 & 90.3 & $E$ & 20.9 & 179 \\
\hline 0.316 & 32.5 & 4 & 148.7 & $\mathrm{NE}$ & 24.2 & 3 & 163.1 & E & 24.8 & 5.99 & 60 & $\mathrm{NE}$ & 24.2 & 330 \\
\hline 0.316 & 32.82 & 3 & 163.1 & $\mathrm{E}$ & 24.8 & 2 & 175.3 & $\mathrm{E}$ & 27.4 & 6.01 & 50.3 & $\mathrm{NE}$ & 23 & 323 \\
\hline 0.316 & 33.76 & 1 & 139 & $\mathrm{NE}$ & 21 & 1 & 143 & $\mathrm{NE}$ & 22 & 1.77 & 14.8 & $\mathrm{~N}$ & 17.6 & 287 \\
\hline 0.316 & 34.08 & 1 & 143 & $\mathrm{NE}$ & 22 & 2 & 116.8 & $\mathrm{NE}$ & 19 & 9.56 & 20.7 & $\mathrm{~N}$ & 18.9 & 113 \\
\hline 0.316 & 44.52 & 1 & 170 & $E$ & 32 & 6 & 156.2 & $\mathrm{NE}$ & 33.4 & 7.56 & 86 & $E$ & 31.9 & 175 \\
\hline 0.316 & 44.83 & 6 & 156.2 & $\mathrm{NE}$ & 33.4 & 5 & 155.3 & $\mathrm{NE}$ & 26.4 & 7.04 & 159.2 & $N$ & 2 & 69 \\
\hline 0.316 & 45.15 & 5 & 155.3 & $\mathrm{NE}$ & 26.4 & 6 & 145.8 & $\mathrm{NE}$ & 24.5 & 4.5 & 33.5 & $\mathrm{NE}$ & 22.9 & 126 \\
\hline 0.316 & 45.47 & 6 & 145.8 & $\mathrm{NE}$ & 24.5 & 4 & 152.4 & $\mathrm{NE}$ & 40.3 & 16.17 & 159.9 & $\mathrm{~N}$ & 6.4 & 251 \\
\hline 0.398 & 1.95 & 1 & 180 & $E$ & 32 & 1 & 165 & $E$ & 37 & 9.83 & 117.8 & SE & 28.9 & 204 \\
\hline 0.398 & 5.54 & 1 & 135 & $\mathrm{NE}$ & 36 & 1 & 112 & $\mathrm{~N}$ & 15 & 22.86 & 147.3 & NW & 8.8 & 60 \\
\hline 0.398 & 7.53 & 1 & 86 & $\mathrm{~N}$ & 33 & 1 & 125 & $\mathrm{NE}$ & 32 & 20.69 & 18.6 & $N$ & 30.9 & 285 \\
\hline 0.398 & 7.93 & 1 & 125 & $\mathrm{NE}$ & 32 & 3 & 156.4 & $\mathrm{NE}$ & 30.6 & 16.24 & 56.4 & $\mathrm{NE}$ & 30.2 & 323 \\
\hline 0.398 & 8.32 & 3 & 156.4 & $\mathrm{NE}$ & 30.6 & 3 & 134.1 & $\mathrm{NE}$ & 34.6 & 12.66 & 76.8 & $\mathrm{E}$ & 30.2 & 165 \\
\hline 0.398 & 8.72 & 3 & 134.1 & $\mathrm{NE}$ & 34.6 & 1 & 173 & $E$ & 18 & 23.21 & 109.1 & E & 16.3 & 15 \\
\hline 0.398 & 10.71 & 1 & 133 & $\mathrm{NE}$ & 39 & 1 & 33 & $\mathrm{~S}$ & 29 & 42.89 & 95.6 & $\mathrm{E}$ & 26.2 & 358 \\
\hline 0.398 & 11.11 & 1 & 33 & $\mathrm{~s}$ & 29 & 1 & 172 & $\mathrm{E}$ & 29 & 19.55 & 102.5 & $\mathrm{E}$ & 27.4 & 195 \\
\hline 0.398 & 11.51 & 1 & 172 & $\mathrm{E}$ & 29 & 3 & 0.9 & $\mathrm{E}$ & 28.7 & 4.31 & 91 & E & 28.7 & 360 \\
\hline 0.398 & 13.1 & 1 & 165 & $\mathrm{E}$ & 35 & 1 & 173 & $\mathrm{E}$ & 30 & 6.58 & 133 & SE & 20.4 & 38 \\
\hline 0.398 & 13.5 & 1 & 173 & E & 30 & 1 & 155 & $\mathrm{NE}$ & 34 & 10.3 & 100.1 & $E$ & 28.9 & 188 \\
\hline 0.398 & 32.21 & 4 & 159.8 & $\mathrm{E}$ & 21.7 & 4 & 153.3 & $\mathrm{NE}$ & 25 & 4.13 & 120.4 & SE & 14.2 & 209 \\
\hline 0.398 & 32.61 & 4 & 153.3 & $\mathrm{NE}$ & 25 & 4 & 170.7 & $\mathrm{E}$ & 25.5 & 7.42 & 67.8 & $\mathrm{E}$ & 24.9 & 337 \\
\hline 0.398 & 33.8 & 1 & 139 & $\mathrm{NE}$ & 21 & 1 & 143 & $\mathrm{NE}$ & 22 & 1.77 & 14.8 & $\mathrm{~N}$ & 17.6 & 287 \\
\hline 0.398 & 34.2 & 1 & 143 & $\mathrm{NE}$ & 22 & 2 & 116.8 & $\mathrm{NE}$ & 19 & 9.56 & 20.7 & $\mathrm{~N}$ & 18.9 & 113 \\
\hline 0.398 & 44.55 & 2 & 168.4 & $E$ & 34 & 7 & 153.2 & $\mathrm{NE}$ & 32.1 & 8.49 & 56.1 & $\mathrm{NE}$ & 31.9 & 149 \\
\hline 0.398 & 44.95 & 7 & 153.2 & $\mathrm{NE}$ & 32.1 & 6 & 159.7 & E & 27.4 & 5.74 & 125.9 & SE & 16.1 & 32 \\
\hline 0.398 & 45.35 & 6 & 159.7 & $\mathrm{E}$ & 27.4 & 7 & 144.1 & $\mathrm{NE}$ & 31.2 & 8.52 & 91.6 & $\mathrm{E}$ & 25.7 & 179 \\
\hline 0.501 & 5.81 & 1 & 135 & $\mathrm{NE}$ & 36 & 1 & 112 & $\mathrm{~N}$ & 15 & 22.86 & 147.3 & NW & 8.8 & 60 \\
\hline 0.501 & 6.32 & 1 & 112 & $\mathrm{~N}$ & 15 & 1 & 179 & $\mathrm{E}$ & 26 & 24.15 & 31.8 & $\mathrm{NE}$ & 14.8 & 301 \\
\hline 0.501 & 7.82 & 2 & 105.2 & $\mathrm{~N}$ & 31 & 3 & 156.4 & $\mathrm{NE}$ & 30.6 & 25.56 & 41.8 & $\mathrm{NE}$ & 28.2 & 308 \\
\hline 0.501 & 8.32 & 3 & 156.4 & $\mathrm{NE}$ & 30.6 & 3 & 134.1 & $\mathrm{NE}$ & 34.6 & 12.66 & 76.8 & E & 30.2 & 165 \\
\hline 0.501 & 8.82 & 3 & 134.1 & $\mathrm{NE}$ & 34.6 & 1 & 173 & $\mathrm{E}$ & 18 & 23.21 & 109.1 & $\mathrm{E}$ & 16.3 & 15 \\
\hline 0.501 & 10.33 & 1 & 134 & $\mathrm{NE}$ & 21 & 1 & 133 & $\mathrm{NE}$ & 39 & 18.01 & 132.1 & SE & 0.7 & 222 \\
\hline 0.501 & 10.83 & 1 & 133 & $\mathrm{NE}$ & 39 & 1 & 33 & $\mathrm{~s}$ & 29 & 42.89 & 95.6 & $E$ & 26.2 & 358 \\
\hline 0.501 & 11.33 & 1 & 33 & $\mathrm{~S}$ & 29 & 4 & 178.7 & E & 28.7 & 16.39 & 104.8 & $E$ & 27.8 & 197 \\
\hline 0.501 & 12.83 & 1 & 165 & $\mathrm{E}$ & 35 & 1 & 173 & $\mathrm{E}$ & 30 & 6.58 & 133 & $\mathrm{SE}$ & 20.4 & 38 \\
\hline 0.501 & 13.33 & 1 & 173 & $\mathrm{E}$ & 30 & 1 & 155 & $\mathrm{NE}$ & 34 & 10.3 & 100.1 & $\mathrm{E}$ & 28.9 & 188 \\
\hline 0.501 & 17.84 & 1 & 4 & $\mathrm{~S}$ & 18 & 1 & 176 & $\mathrm{E}$ & 13 & 5.43 & 22.5 & $\mathrm{~N}$ & 5.9 & 113 \\
\hline 0.501 & 18.34 & 1 & 176 & $E$ & 13 & 1 & 165 & $\mathrm{E}$ & 20 & 7.64 & 147.2 & SE & 6.3 & 237 \\
\hline 0.501 & 32.38 & 5 & 155.9 & $\mathrm{NE}$ & 22 & 5 & 160.1 & $\mathrm{E}$ & 24.7 & 3.14 & 8 & $\mathrm{~N}$ & 12.1 & 279 \\
\hline 0.501 & 32.88 & 5 & 160.1 & $\mathrm{E}$ & 24.7 & 2 & 175.3 & $\mathrm{E}$ & 27.4 & 7.2 & 53.3 & $\mathrm{NE}$ & 23.8 & 325 \\
\hline 0.501 & 33.38 & 2 & 175.3 & $\mathrm{E}$ & 27.4 & 1 & 139 & $\mathrm{NE}$ & 21 & 15.94 & 42.6 & $\mathrm{NE}$ & 20.9 & 136 \\
\hline 0.501 & 33.88 & 1 & 139 & $\mathrm{NE}$ & 21 & 3 & 128.3 & $\mathrm{NE}$ & 19.8 & 3.91 & 24.9 & $\mathrm{NE}$ & 19.3 & 116 \\
\hline 0.501 & 44.91 & 8 & 156.9 & $\mathrm{NE}$ & 33 & 8 & 156.9 & NE & 24.1 & 8.81 & 156.9 & $\mathrm{SE}$ & 0 & 67 \\
\hline 0.501 & 45.41 & 8 & 156.9 & $\mathrm{NE}$ & 24.1 & 6 & 146.2 & $\mathrm{NE}$ & 36 & 12.95 & 129.8 & $\mathrm{SE}$ & 11.5 & 218 \\
\hline 0.631 & 4.24 & 1 & 1 & $\mathrm{~s}$ & 43 & 1 & 2 & $\mathrm{~s}$ & 35 & 8.02 & 178 & $\mathrm{~s}$ & 2.8 & 87 \\
\hline 0.631 & 4.87 & 1 & 2 & $\mathrm{~s}$ & 35 & 1 & 135 & $\mathrm{NE}$ & 36 & 26.79 & 70.9 & $\mathrm{E}$ & 33.2 & 164 \\
\hline 0.631 & 5.5 & 1 & 135 & $\mathrm{NE}$ & 36 & 1 & 112 & $\mathrm{~N}$ & 15 & 22.86 & 147.3 & NW & 8.8 & 60 \\
\hline 0.631 & 6.13 & 1 & 112 & $\mathrm{~N}$ & 15 & 1 & 179 & $E$ & 26 & 24.15 & 31.8 & $\mathrm{NE}$ & 14.8 & 301 \\
\hline 0.631 & 6.77 & 1 & 179 & $\mathrm{E}$ & 26 & 1 & 86 & $\mathrm{~N}$ & 33 & 42.16 & 50.2 & $\mathrm{NE}$ & 20.8 & 143 \\
\hline 0.631 & 7.4 & 1 & 86 & $\mathrm{~N}$ & 33 & 2 & 133.3 & $\mathrm{NE}$ & 30.7 & 24.52 & 25.4 & $\mathrm{NE}$ & 29.5 & 291 \\
\hline 0.631 & 8.03 & 2 & 133.3 & $\mathrm{NE}$ & 30.7 & 5 & 145 & $\mathrm{NE}$ & 32.6 & 6.41 & 29.6 & $\mathrm{NE}$ & 30 & 301 \\
\hline 0.631 & 8.66 & 5 & 145 & $\mathrm{NE}$ & 32.6 & 1 & 173 & $\mathrm{E}$ & 18 & 18.51 & 121.5 & $\mathrm{SE}$ & 14.3 & 28 \\
\hline 0.631 & 9.29 & 1 & 173 & $E$ & 18 & 1 & 134 & $\mathrm{NE}$ & 21 & 13.11 & 76.7 & $\mathrm{E}$ & 17.9 & 167 \\
\hline 0.631 & 9.92 & 1 & 134 & $\mathrm{NE}$ & 21 & 1 & 133 & $\mathrm{NE}$ & 39 & 18.01 & 132.1 & SE & 0.7 & 222 \\
\hline 0.631 & 10.55 & 1 & 133 & $\mathrm{NE}$ & 39 & 1 & 33 & $\mathrm{~s}$ & 29 & 42.89 & 95.6 & $\mathrm{E}$ & 26.2 & 358 \\
\hline 0.631 & 11.18 & 1 & 33 & $\mathrm{~S}$ & 29 & 4 & 178.7 & $E$ & 28.7 & 16.39 & 104.8 & $\mathrm{E}$ & 27.8 & 197 \\
\hline 0.631 & 13.08 & 1 & 165 & $E$ & 35 & 1 & 173 & $E$ & 30 & 6.58 & 133 & SE & 20.4 & 38 \\
\hline 0.631 & 13.71 & 1 & 173 & $E$ & 30 & 1 & 155 & $\mathrm{NE}$ & 34 & 10.3 & 100.1 & $\mathrm{E}$ & 28.9 & 188 \\
\hline 0.631 & 14.34 & 1 & 155 & $\mathrm{NE}$ & 34 & 1 & 155 & $\mathrm{NE}$ & 35 & 1 & 155 & SE & 0 & 245 \\
\hline 0.631 & 17.49 & 1 & 4 & $\mathrm{~s}$ & 18 & 1 & 176 & $\mathrm{E}$ & 13 & 5.43 & 22.5 & $\mathrm{~N}$ & 5.9 & 113 \\
\hline 0.631 & 18.12 & 1 & 176 & $E$ & 13 & 1 & 165 & $E$ & 20 & 7.64 & 147.2 & SE & 6.3 & 237 \\
\hline 0.631 & 32 & 1 & 165 & $E$ & 25 & 7 & 154.9 & NE & 23.1 & 4.5 & 43.8 & $\mathrm{NE}$ & 21.7 & 136 \\
\hline 0.631 & 32.63 & 7 & 154.9 & $\mathrm{NE}$ & 23. & 4 & 170.7 & $\mathrm{E}$ & 25.5 & 6.89 & 51.5 & $\mathrm{NE}$ & 22.6 & 322 \\
\hline 0.631 & 33.27 & 4 & 170.7 & $\mathrm{E}$ & 25.5 & 1 & 139 & NE & 21 & 13.12 & 44.1 & $\mathrm{NE}$ & 20.9 & 137 \\
\hline 0.631 & 33.9 & 1 & 139 & $\mathrm{NE}$ & 21 & 3 & 128.3 & $\mathrm{NE}$ & 19.8 & 3.91 & 24.9 & $\mathrm{NE}$ & 19.3 & 116 \\
\hline 0.631 & 44.62 & 3 & 165.5 & $\mathrm{E}$ & 34. & 1. & 154.7 & NE & 29.3 & 8.01 & 21.1 & $N$ & 22.1 & 117 \\
\hline 0.631 & 45.25 & 11 & 154.7 & $\mathrm{NE}$ & 29.3 & 8 & 146.8 & $\mathrm{NE}$ & 30.9 & 4.22 & 85.2 & $\mathrm{E}$ & 27.7 & 173 \\
\hline 0.794 & 2.2 & 1 & 180 & $E$ & 32 & 1 & 165 & $E$ & 37 & 9.83 & 117.8 & $\mathrm{SE}$ & 28.9 & 204 \\
\hline 0.794 & 3 & 1 & 165 & $\mathrm{E}$ & 37 & 1 & 1 & $\mathrm{~s}$ & 43 & 11.87 & 45.9 & $\mathrm{NE}$ & 33.4 & 321 \\
\hline 0.794 & 3.79 & 1 & 1 & $\mathrm{~S}$ & 43 & 1 & 2 & $\mathrm{~s}$ & 35 & 8.02 & 178 & $\mathrm{~s}$ & 2.8 & 87 \\
\hline 0.794 & 6.17 & 2 & 128 & $\mathrm{NE}$ & 25 & 1 & 179 & $\mathrm{E}$ & 26 & 21.42 & 61.1 & $\mathrm{NE}$ & 23.3 & 329 \\
\hline 0.794 & 6.97 & 1 & 179 & $E$ & 26 & 2 & 105.2 & $\mathrm{~N}$ & 31 & 33.53 & 60 & $\mathrm{NE}$ & 23.1 & 152 \\
\hline 0.794 & 7.76 & 2 & 105.2 & $\mathrm{~N}$ & 31 & 5 & 146.9 & $\mathrm{NE}$ & 31.6 & 21.31 & 34.1 & $\mathrm{NE}$ & 29.6 & 302 \\
\hline 0.794 & 8.56 & 5 & 146.9 & $\mathrm{NE}$ & 31. & 2 & 147.4 & $\mathrm{NE}$ & 25.3 & 6.35 & 145 & $\mathrm{SE}$ & 1.2 & 55 \\
\hline 0.794 & 9.35 & 2 & 147.4 & $\mathrm{NE}$ & 25. & 1 & 134 & $\mathrm{NE}$ & 21 & 6.79 & 9.4 & $\mathrm{~N}$ & $\begin{array}{r}17.5 \\
\end{array}$ & 102 \\
\hline
\end{tabular}




\begin{tabular}{|c|c|c|c|c|c|c|c|c|c|c|c|c|c|c|}
\hline 1.259 & 0.82 & 1 & 159 & $E$ & 25 & 1 & 180 & $E$ & 32 & 12.13 & 41.4 & $\mathrm{NE}$ & 22.5 & 314 \\
\hline 1.259 & 2.08 & 1 & 180 & $E$ & 32 & 1 & 165 & $E$ & 37 & 9.83 & 117.8 & SE & 28.9 & 204 \\
\hline 1.259 & 3.34 & 1 & 165 & $\mathrm{E}$ & 37 & 2 & 1.5 & $E$ & 39 & 10.3 & 69.3 & $\mathrm{E}$ & 36.9 & 340 \\
\hline 1.259 & 4.6 & 2 & 1.5 & $E$ & 39 & 1 & 135 & $\mathrm{NE}$ & 36 & 27.93 & 61 & $\mathrm{NE}$ & 34.9 & 157 \\
\hline 1.259 & 5.86 & 1 & 135 & $\mathrm{NE}$ & 36 & 2 & 155.2 & $\mathrm{NE}$ & 17.6 & 20.33 & 120.8 & SE & 10.1 & 27 \\
\hline 1.259 & 7.12 & 2 & 155.2 & $\mathrm{NE}$ & 17.6 & 6 & 135.7 & $\mathrm{NE}$ & 30.6 & 15.1 & 115.8 & SE & 11.3 & 204 \\
\hline 1.259 & 8.38 & 6 & 135.7 & $\mathrm{NE}$ & 30.6 & 3 & 143.6 & $\mathrm{NE}$ & 26.2 & 5.78 & 102.7 & $E$ & 17.8 & 9 \\
\hline 1.259 & 9.64 & 3 & 143.6 & $\mathrm{NE}$ & 26.2 & 3 & 156.1 & $\mathrm{NE}$ & 24.5 & 5.62 & 78.9 & $\mathrm{E}$ & 23.9 & 347 \\
\hline 1.259 & 10.9 & 3 & 156.1 & $\mathrm{NE}$ & 24.5 & 4 & 178.7 & $\mathrm{E}$ & 28.7 & 10.86 & 52.5 & $\mathrm{NE}$ & 23.9 & 324 \\
\hline 1.259 & 12.15 & 4 & 178.7 & $E$ & 28.7 & 2 & 168.7 & $\mathrm{E}$ & 32.4 & 6.26 & 124.1 & SE & 24.1 & 210 \\
\hline 1.259 & 13.41 & 2 & 168.7 & $\bar{E}$ & 32.4 & 2 & 155 & $\mathrm{NE}$ & 34.5 & 7.83 & 89.9 & $\mathrm{E}$ & 31.9 & 178 \\
\hline 1.259 & 18.45 & 2 & 0.6 & $E$ & 15.5 & 1 & 165 & $E$ & 20 & 6.54 & 127.6 & SE & 12.5 & 216 \\
\hline 1.259 & 19.71 & 1 & 165 & $E$ & 20 & 1 & 151 & $\mathrm{NE}$ & 28 & 9.77 & 124.7 & SE & 13.2 & 213 \\
\hline 1.259 & 31.04 & 1 & 145 & NE & 34 & 4 & 159.8 & $E$ & 21.7 & 13.99 & 125.6 & SE & 12.6 & 32 \\
\hline 1.259 & 32.3 & 4 & 159.8 & $E$ & 21.7 & 8 & 162.1 & $\mathrm{E}$ & 25 & 3.36 & 175.5 & $\mathrm{~N}$ & 6.2 & 266 \\
\hline 1.259 & 33.56 & 8 & 162.1 & $\bar{E}$ & 25 & 4 & 131.5 & $\mathrm{NE}$ & 20.1 & 12.51 & 33.1 & $\mathrm{NE}$ & 19.9 & 126 \\
\hline 1.259 & 44.89 & 8 & 156.9 & $\mathrm{NE}$ & 33 & 14 & 151.5 & $\mathrm{NE}$ & 29 & 4.83 & 5.3 & $\mathrm{~N}$ & 17.2 & 99 \\
\hline 1.585 & 1.46 & 1 & 159 & $\mathrm{E}$ & 25 & 2 & 172 & $\mathrm{E}$ & 34.3 & 11.24 & 16.8 & $\mathrm{~N}$ & 16 & 289 \\
\hline 1.585 & 3.04 & 2 & 172 & $E$ & 34.3 & 2 & 1.5 & $\mathrm{E}$ & 39 & 7.34 & 40.5 & $\mathrm{NE}$ & 27 & 316 \\
\hline 1.585 & 4.63 & 2 & 1.5 & $E$ & 39 & 2 & 128 & $\mathrm{NE}$ & 25.1 & 30.37 & 36.8 & $\mathrm{NE}$ & 25.1 & 134 \\
\hline 1.585 & 6.21 & 2 & 128 & $\mathrm{NE}$ & 25.1 & 3 & 125.2 & $\mathrm{NE}$ & 25.3 & 1.2 & 48 & $\mathrm{NE}$ & 24.8 & 136 \\
\hline 1.585 & 7.8 & 3 & 125.2 & $\mathrm{NE}$ & 25.3 & 7 & 147 & $\mathrm{NE}$ & 29.9 & 10.96 & 19.5 & $\mathrm{~N}$ & 24.5 & 291 \\
\hline 1.585 & 9.38 & 7 & 147 & $\mathrm{NE}$ & 29.9 & 3 & 156.1 & $\mathrm{NE}$ & 24.5 & 6.8 & 116.7 & SE & 16.1 & 23 \\
\hline 1.585 & 10.97 & 3 & 156.1 & $\mathrm{NE}$ & 24.5 & 4 & 178.7 & $E$ & 28.7 & 10.86 & 52.5 & $\mathrm{NE}$ & 23.9 & 324 \\
\hline 1.585 & 12.55 & 4 & 178.7 & $E$ & 28.7 & 3 & 164 & $E$ & 32.8 & 8.49 & 113.5 & SE & 26.4 & 201 \\
\hline 1.585 & 14.14 & 3 & 164 & $\bar{E}$ & 32.8 & 1 & 155 & $\mathrm{NE}$ & 35 & 5.49 & 97.3 & $E$ & 30.6 & 184 \\
\hline 1.585 & 18.89 & 3 & 174.5 & $E$ & 16.8 & 1 & 151 & $\mathrm{NE}$ & 28 & 14.13 & 125.6 & SE & 12.9 & 214 \\
\hline 1.585 & 31.57 & 1 & 145 & NE & 34 & 12 & 161.4 & $E$ & 23.9 & 12.76 & 118.4 & SE & 16.8 & 24 \\
\hline 1.585 & 33.16 & 12 & 161.4 & $E$ & 23.9 & 4 & 131.5 & $\mathrm{NE}$ & 20.1 & 11.66 & 36.8 & $\mathrm{NE}$ & 20 & 129 \\
\hline 1.995 & 1.9 & 1 & 159 & $E$ & 25 & 3 & 175.4 & $E$ & 37.1 & 14.67 & 18.5 & $\mathrm{~N}$ & 16.5 & 291 \\
\hline 1.995 & 3.9 & 3 & 175.4 & $E$ & 37.1 & 3 & 149.4 & $\mathrm{NE}$ & 25.9 & 17.4 & 29.1 & $\mathrm{NE}$ & 22.8 & 125 \\
\hline 1.995 & 5.9 & 3 & 149.4 & $\mathrm{NE}$ & 25.9 & 3 & 125.2 & $\mathrm{NE}$ & 25.3 & 10.39 & 43.8 & $\mathrm{NE}$ & 25.1 & 135 \\
\hline 1.995 & 7.89 & 3 & 125.2 & $\mathrm{NE}$ & 25.3 & 8 & 145.7 & $\mathrm{NE}$ & 28.7 & 9.82 & 23.9 & $\mathrm{NE}$ & 24.9 & 294 \\
\hline 1.995 & 9.89 & 8 & 145.7 & $\mathrm{NE}$ & 28.7 & 6 & 175.5 & $E$ & 28.2 & 14.07 & 72.7 & $E$ & 27.6 & 341 \\
\hline 1.995 & 11.88 & 6 & 175.5 & $\mathrm{E}$ & 28.2 & 3 & 164 & $\bar{E}$ & 32.8 & 7.4 & 122 & SE & 23.3 & 209 \\
\hline 1.995 & 13.88 & 3 & 164 & $\mathrm{E}$ & 32.8 & 1 & 155 & $\mathrm{NE}$ & 35 & 5.49 & 97.3 & $E$ & 30.6 & 184 \\
\hline 1.995 & 15.87 & 1 & 155 & $\mathrm{NE}$ & 35 & 1 & 4 & $S$ & 18 & 20.92 & 134.3 & SE & 13.9 & 40 \\
\hline 1.995 & 17.87 & 1 & 4 & $\mathrm{~s}$ & 18 & 2 & 169.4 & $\mathrm{E}$ & 16.4 & 4.59 & 66 & $\mathrm{NE}$ & 16 & 157 \\
\hline 1.995 & 19.86 & 2 & 169.4 & $\mathrm{E}$ & 16.4 & 1 & 151 & $\mathrm{NE}$ & 28 & 13.36 & 130.8 & SE & 10.4 & 220 \\
\hline 1.995 & 29.84 & 1 & 161 & $\bar{E}$ & 17 & 1 & 145 & $\mathrm{NE}$ & 34 & 18.2 & 132.5 & $\mathrm{SE}$ & 8.3 & 221 \\
\hline 1.995 & 31.83 & 1 & 145 & $\mathrm{NE}$ & 34 & 13 & 159.8 & $E$ & 23.6 & 12.57 & 121.1 & SE & 15.3 & 27 \\
\hline 1.995 & 33.83 & 13 & 159.8 & $E$ & 23.6 & 3 & 128.3 & $\mathrm{NE}$ & 19.8 & 12.09 & 35.4 & $\mathrm{NE}$ & 19.8 & 127 \\
\hline 2.512 & 1.5 & 1 & 159 & $\mathrm{E}$ & 25 & 3 & 175.4 & $\mathrm{E}$ & 37.1 & 14.67 & 18.5 & $N$ & 16.5 & 291 \\
\hline 2.512 & 4.02 & 3 & 175.4 & $E$ & 37.1 & 3 & 149.4 & $\mathrm{NE}$ & 25.9 & 17.4 & 29.1 & $\mathrm{NE}$ & 22.8 & 125 \\
\hline 2.512 & 6.53 & 3 & 149.4 & $\mathrm{NE}$ & 25.9 & 9 & 139.6 & $\mathrm{NE}$ & 29.7 & 5.89 & 97.5 & $E$ & 20.9 & 185 \\
\hline 2.512 & 9.04 & 9 & 139.6 & $\mathrm{NE}$ & 29.7 & 5 & 163.3 & $E$ & 23.8 & 12.05 & 92.7 & E & 22.6 & 359 \\
\hline 2.512 & 11.55 & 5 & 163.3 & $E$ & 23.8 & 6 & 171.9 & $\bar{E}$ & 30.5 & 7.74 & 15.4 & $\mathrm{~N}$ & 13.2 & 288 \\
\hline 2.512 & 14.06 & 6 & 171.9 & $\mathrm{E}$ & 30.5 & 1 & 155 & $\mathrm{NE}$ & 35 & 10.17 & 103.6 & $\mathrm{E}$ & 28.7 & 191 \\
\hline 2.512 & 16.58 & 1 & 155 & $\mathrm{NE}$ & 35 & 3 & 174.5 & $E$ & 16.8 & 19.85 & 141.3 & SE & 9.4 & 49 \\
\hline 2.512 & 19.09 & 3 & 174.5 & $\mathrm{E}$ & 16.8 & 1 & 151 & $\mathrm{NE}$ & 28 & 14.13 & 125.6 & SE & 12.9 & 214 \\
\hline 2.512 & 26.62 & 1 & 148 & $\mathrm{NE}$ & 17 & 1 & 161 & $E$ & 17 & 3.79 & 64.5 & $\mathrm{NE}$ & 16.9 & 334 \\
\hline 2.512 & 29.13 & 1 & 161 & $E$ & 17 & 1 & 145 & $\mathrm{NE}$ & 34 & 18.2 & 132.5 & SE & 8.3 & 221 \\
\hline 2.512 & 31.65 & 1 & 145 & NE & 34 & 14 & 158.7 & $\mathrm{E}$ & 23.4 & 12.42 & 123 & SE & 14.2 & 29 \\
\hline 2.512 & 34.16 & 14 & 158.7 & $E$ & 23.4 & 2 & 116.8 & $\mathrm{NE}$ & 19 & 15.41 & 31.1 & $\mathrm{NE}$ & 18.9 & 124 \\
\hline 3.162 & 2.3 & 3 & 168.5 & $\mathrm{E}$ & 31.1 & 2 & 1.5 & $\mathrm{E}$ & 39 & 10.86 & 32.7 & $\mathrm{NE}$ & 22.8 & 307 \\
\hline 3.162 & 5.46 & 2 & 1.5 & $E$ & 39 & 11 & 137.7 & $\mathrm{NE}$ & 28.8 & 25.84 & 44.1 & $\mathrm{NE}$ & 28.7 & 141 \\
\hline 3.162 & 8.62 & 11 & 137.7 & $\mathrm{NE}$ & 28.8 & 8 & 171.1 & $\mathrm{E}$ & 25.4 & 15.41 & 77.9 & $E$ & 25.4 & 345 \\
\hline 3.162 & 11.79 & 8 & 171.1 & $E$ & 25.4 & 4 & 161.7 & $\mathrm{E}$ & 33.3 & 9.09 & 139 & SE & 14.2 & 227 \\
\hline 3.162 & 14.95 & 4 & 161.7 & $\mathrm{E}$ & 33.3 & 2 & 0.6 & $E$ & 15.5 & 19.25 & 148.8 & $\mathrm{SE}$ & 8.3 & 56 \\
\hline 3.162 & 18.11 & 2 & 0.6 & $E$ & 15.5 & 2 & 156.9 & $\mathrm{NE}$ & 23.8 & 11.42 & 126.4 & SE & 12.7 & 215 \\
\hline 3.162 & 27.6 & 1 & 148 & $\mathrm{NE}$ & 17 & 2 & 150.5 & $\mathrm{NE}$ & 25.3 & 8.35 & 155 & NW & 2.1 & 245 \\
\hline 3.162 & 30.76 & 2 & 150.5 & $\mathrm{NE}$ & 25.3 & 14 & 158.7 & $\mathrm{E}$ & 23.4 & 3.89 & 96.2 & $\mathrm{E}$ & 21 & 4 \\
\hline 3.162 & 33.92 & 14 & 158.7 & $\mathrm{E}$ & 23.4 & 2 & 116.8 & NE & 19 & 15.41 & 31.1 & NE & 18.9 & 124 \\
\hline 3.981 & 2.95 & 3 & 168.5 & $E$ & 31.1 & 5 & 164.4 & $E$ & 28.6 & 3.14 & 22.4 & $\mathrm{~N}$ & 18.6 & 116 \\
\hline 3.981 & 6.93 & 5 & 164.4 & $E$ & 28.6 & 12 & 141.7 & $\mathrm{NE}$ & 27.8 & 10.71 & 58.3 & NE & 27.7 & 150 \\
\hline 3.981 & 10.91 & 12 & 141.7 & $\mathrm{NE}$ & 27.8 & 8 & 169.6 & $E$ & 30.7 & 13.81 & 52.2 & $\mathrm{NE}$ & 27.8 & 322 \\
\hline 3.981 & 14.89 & 8 & 169.6 & $E$ & 30.7 & 3 & 174.5 & $\mathrm{E}$ & 16.8 & 14.04 & 164.5 & $\mathrm{~S}$ & 3 & 74 \\
\hline 3.981 & 18.87 & 3 & 174.5 & $E$ & 16.8 & 1 & 151 & $\mathrm{NE}$ & 28 & 14.13 & 125.6 & SE & 12.9 & 214 \\
\hline 3.981 & 22.86 & 1 & 151 & $\mathrm{NE}$ & 28 & 1 & 148 & $\mathrm{NE}$ & 17 & 11.06 & 155 & NW & 2.1 & 66 \\
\hline 3.981 & 26.84 & 1 & 148 & $\mathrm{NE}$ & 17 & 2 & 150.5 & $\mathrm{NE}$ & 25.3 & 8.35 & 155 & NW & 2.1 & 245 \\
\hline 3.981 & 30.82 & 2 & 150.5 & $\mathrm{NE}$ & 25.3 & 16 & 155.5 & $\mathrm{NE}$ & 22.7 & 3.33 & 117.6 & SE & 14.4 & 25 \\
\hline 5.012 & 2.81 & 3 & 168.5 & $\mathrm{E}$ & 31.1 & 7 & 147.3 & $\mathrm{NE}$ & 26.6 & 11.09 & 41.6 & $\mathrm{NE}$ & 25.7 & 136 \\
\hline 5.012 & 7.82 & 7 & 147.3 & $\mathrm{NE}$ & 26.6 & 15 & 158.6 & $E$ & 28.2 & 5.46 & 43.4 & $\mathrm{NE}$ & 25.9 & 315 \\
\hline 5.012 & 12.83 & 15 & 158.6 & $E$ & 28.2 & 4 & 164.2 & $E$ & 28.8 & 2.73 & 56.8 & $\mathrm{NE}$ & 27.7 & 328 \\
\hline 5.012 & 17.84 & 4 & 164.2 & $E$ & 28.8 & 3 & 161.1 & $E$ & 20 & 8.88 & 170.2 & $\mathrm{~N}$ & 3.3 & 81 \\
\hline 5.012 & 22.86 & 3 & 161.1 & $\bar{E}$ & 20 & 1 & 148 & $\mathrm{NE}$ & 17 & 5.13 & 26.9 & $\mathrm{NE}$ & 14.7 & 119 \\
\hline 5.012 & 27.87 & 1 & 148 & $\mathrm{NE}$ & 17 & 12 & 156.8 & $\mathrm{NE}$ & 23.7 & 7.3 & 175.8 & $\mathrm{~N}$ & 8.1 & 267 \\
\hline 5.012 & 32.88 & 12 & 156.8 & $\mathrm{NE}$ & 23.7 & 6 & 150.1 & $\mathrm{NE}$ & 21.4 & 3.37 & 20.4 & $\mathrm{~N}$ & 16.8 & 112 \\
\hline 6.31 & 3.93 & 4 & 172.3 & $\mathrm{E}$ & $33.9 \|$ & 14 & 142.9 & $\mathrm{NE}$ & 27.1 & 16.24 & 40.4 & $\mathrm{NE}$ & 26.6 & 136 \\
\hline
\end{tabular}




\begin{tabular}{|c|c|c|c|c|c|c|c|c|c|c|c|c|c|c|}
\hline $\begin{array}{l}\text { window size } \\
(\mathrm{m})\end{array}$ & $\begin{array}{c}\text { elevation between } \\
\text { two consecutive windows } \\
(\mathrm{m})\end{array}$ & $\left|\begin{array}{c}\text { lower window } \\
\text { number } \\
\text { of measures }\end{array}\right|$ & $\begin{array}{c}\text { average } \\
\text { orientation }\end{array}$ & & & $\begin{array}{c}\text { upper window } \\
\text { number } \\
\text { of measures }\end{array}$ & $\begin{array}{l}\text { average } \\
\text { orientation }\end{array}$ & & & $\mid \begin{array}{c}\text { tilt angle } \\
\left({ }^{\circ}\right)\end{array}$ & tilt axis & & & $\begin{array}{c}\text { tilt way } \\
\left({ }^{\circ}\right)\end{array}$ \\
\hline 0.02 & 56.1 & 1 & 115 & NE & 26 & 1 & 141 & $\mathrm{NE}$ & 33 & 14.45 & 6.4 & $\mathrm{~N}$ & 24.8 & 278 \\
\hline 0.025 & 55.46 & 1 & 145 & $\mathrm{NE}$ & 37 & 1 & 172 & $E$ & 26 & 17.66 & 110.2 & E & 23.3 & 14 \\
\hline 0.025 & 56.11 & 1 & 115 & NE & 26 & 1 & 141 & $\mathrm{NE}$ & 33 & 14.45 & 6.4 & $\mathrm{~N}$ & 24.8 & 278 \\
\hline 0.032 & 55.46 & 1 & 145 & $\mathrm{NE}$ & 37 & 1 & 172 & $\mathrm{E}$ & 26 & 17.66 & 110.2 & $\mathrm{E}$ & 23.3 & 14 \\
\hline 0.032 & 55.75 & 1 & 175 & $\mathrm{E}$ & 16 & 1 & 151 & $\mathrm{NE}$ & 23 & 10.51 & 115.3 & $\mathrm{SE}$ & 13.9 & 204 \\
\hline 0.032 & 55.94 & 1 & 159 & $\mathrm{E}$ & 29 & 1 & 165 & $\mathrm{E}$ & 30 & 3.12 & 50.8 & $\mathrm{NE}$ & 27.8 & 323 \\
\hline 0.032 & 56.1 & 1 & 115 & $\mathrm{NE}$ & 26 & 1 & 141 & $\mathrm{NE}$ & 33 & 14.45 & 6.4 & $\mathrm{~N}$ & 24.8 & 278 \\
\hline 0.04 & 55.16 & 1 & 170 & E & 32 & 1 & 167 & $\mathrm{E}$ & 36 & 4.34 & 149.3 & SE & 12.5 & 236 \\
\hline 0.04 & 55.28 & 1 & 160 & $\mathrm{E}$ & 37 & 1 & 145 & $\mathrm{NE}$ & 34 & 9.19 & 39.7 & $\mathrm{NE}$ & 33 & 135 \\
\hline 0.04 & 55.4 & 1 & 152 & $\mathrm{NE}$ & 33 & 1 & 145 & $\mathrm{NE}$ & 37 & 5.66 & 109 & $\mathrm{E}$ & 23.9 & 194 \\
\hline 0.04 & 55.44 & 1 & 145 & $\mathrm{NE}$ & 37 & 1 & 172 & $\mathrm{E}$ & 26 & 17.66 & 110.2 & $\mathrm{E}$ & 23.3 & 14 \\
\hline 0.04 & 55.64 & 1 & 155 & NE & 28 & 1 & 156 & $\mathrm{NE}$ & 34 & 6.02 & 159.7 & $\mathrm{~N}$ & 2.5 & 250 \\
\hline 0.04 & 55.76 & 1 & 175 & E & 16 & 1 & 151 & $\mathrm{NE}$ & 23 & 10.51 & 115.3 & $\mathrm{SE}$ & 13.9 & 204 \\
\hline 0.04 & 55.88 & 1 & 158 & $\mathrm{E}$ & 33 & 1 & 159 & $\mathrm{E}$ & 29 & 4.03 & 152.2 & $\mathrm{SE}$ & 3.8 & 61 \\
\hline 0.04 & 56 & 1 & 165 & $\mathrm{E}$ & 30 & 1 & 48 & NW & 8 & 34.31 & 176.1 & $\mathrm{~N}$ & 6.3 & 88 \\
\hline 0.04 & 56.12 & 1 & 115 & NE & 26 & 1 & 141 & $\mathrm{NE}$ & 33 & 14.45 & 6.4 & $\mathrm{~N}$ & 24.8 & 278 \\
\hline 0.04 & 56.16 & 1 & 141 & $\mathrm{NE}$ & 33 & 1 & 152 & $\mathrm{NE}$ & 53 & 21.3 & 162.2 & $\mathrm{~N}$ & 13.2 & 256 \\
\hline 0.04 & 56.28 & 1 & 140 & $\mathrm{NE}$ & 52 & 1 & 167 & $\mathrm{E}$ & 30 & 27.84 & 121.1 & $\mathrm{SE}$ & 22.5 & 21 \\
\hline 0.05 & 11.43 & 1 & 178 & $\mathrm{E}$ & 33 & 1 & 180 & $\mathrm{E}$ & 22 & 11.04 & 174.7 & $\mathrm{~s}$ & 2.1 & 84 \\
\hline 0.05 & 11.78 & 1 & 177 & $\mathrm{E}$ & 53 & 1 & 8 & $\mathrm{~s}$ & 29 & 24.98 & 169.3 & $\mathrm{~s}$ & 10.1 & 74 \\
\hline 0.05 & 12.03 & 1 & 150 & NE & 28 & 1 & 158 & $\mathrm{E}$ & 11 & 17.17 & 145.4 & SE & 2.4 & 55 \\
\hline 0.05 & 55.18 & 1 & 170 & $\mathrm{E}$ & 32 & 1 & 167 & $\mathrm{E}$ & 36 & 4.34 & 149.3 & $\mathrm{SE}$ & 12.5 & 236 \\
\hline 0.05 & 55.23 & 1 & 167 & $\mathrm{E}$ & 36 & 1 & 160 & $\mathrm{E}$ & 37 & 4.28 & 90.1 & $\mathrm{E}$ & 35.3 & 178 \\
\hline 0.05 & 55.28 & 1 & 160 & $\mathrm{E}$ & 37 & 1 & 145 & $\mathrm{NE}$ & 34 & 9.19 & 39.7 & $\mathrm{NE}$ & 33 & 135 \\
\hline 0.05 & 55.43 & 1 & 152 & NE & 33 & 2 & 156.3 & $\mathrm{NE}$ & 30.8 & 3.17 & 112.5 & $\mathrm{SE}$ & 22.4 & 18 \\
\hline 0.05 & 55.58 & 1 & 148 & NE & 32 & 1 & 155 & $\mathrm{NE}$ & 28 & 5.31 & 114.3 & $\mathrm{SE}$ & 19.1 & 20 \\
\hline 0.05 & 55.63 & 1 & 155 & $\mathrm{NE}$ & 28 & 1 & 156 & $\mathrm{NE}$ & 34 & $\begin{array}{l}6.02 \\
6.02\end{array}$ & $\begin{array}{l}159.7\end{array}$ & $\mathrm{~N}$ & 2.5 & 250 \\
\hline 0.05 & 55.68 & 1 & 156 & $\mathrm{NE}$ & 34 & 1 & 175 & $\mathrm{E}$ & 16 & 19.5 & 143 & $\mathrm{SE}$ & 8.6 & 50 \\
\hline 0.05 & 55.73 & 1 & 175 & $\mathrm{E}$ & 16 & 1 & 151 & $\mathrm{NE}$ & 23 & 10.51 & 115.3 & $\mathrm{SE}$ & 13.9 & 204 \\
\hline 0.05 & 55.88 & 1 & 158 & $\mathrm{E}$ & 33 & 1 & 159 & $\mathrm{E}$ & 29 & 4.03 & 152.2 & $\mathrm{SE}$ & 3.8 & 61 \\
\hline 0.05 & 55.93 & 1 & 159 & $\mathrm{E}$ & 29 & 1 & 165 & $\mathrm{E}$ & 30 & 3.12 & 50.8 & $\mathrm{NE}$ & 27.8 & 323 \\
\hline 0.05 & 55.98 & 1 & 165 & $\mathrm{E}$ & 30 & 1 & 48 & NW & 8 & 34.31 & 176.1 & $\mathrm{~N}$ & 6.3 & 88 \\
\hline 0.05 & 56.23 & 1 & 152 & $\mathrm{NE}$ & 53 & 1 & 140 & $\mathrm{NE}$ & 52 & 9.57 & 46.2 & $\mathrm{NE}$ & 51.9 & 142 \\
\hline 0.05 & 56.28 & 1 & 140 & $\mathrm{NE}$ & 52 & 1 & 167 & $\mathrm{E}$ & 30 & 27.84 & 121.1 & $\mathrm{SE}$ & 22.5 & 21 \\
\hline 0.05 & 56.33 & 1 & 167 & $\mathrm{E}$ & 30 & 1 & 158 & $\mathrm{E}$ & 28 & 4.79 & 44.9 & $\mathrm{NE}$ & 26.1 & 138 \\
\hline 0.063 & 11.34 & 1 & 152 & $\mathrm{NE}$ & 25 & 1 & 178 & $\mathrm{E}$ & 33 & 14.77 & 39.6 & $\mathrm{NE}$ & 23.3 & 312 \\
\hline 0.063 & 11.53 & 1 & 180 & $\mathrm{E}$ & 22 & 1 & 163 & $\mathrm{E}$ & 41 & 20.81 & 149.3 & $\mathrm{SE}$ & 11.7 & 237 \\
\hline 0.063 & 11.6 & 1 & 163 & $\mathrm{E}$ & 41 & 1 & 162 & $\mathrm{E}$ & 32 & 9.02 & 165.6 & $\mathrm{~N}$ & 2.2 & 76 \\
\hline 0.063 & 11.79 & 1 & 177 & $\mathrm{E}$ & 53 & 1 & 8 & $\mathrm{~s}$ & 29 & 24.98 & 169.3 & $\mathrm{~s}$ & 10.1 & 74 \\
\hline 0.063 & 11.85 & 1 & 8 & $\mathrm{~S}$ & 29 & 1 & 180 & $\mathrm{E}$ & 42 & 13.78 & 167.6 & $\mathrm{~s}$ & 10.9 & 255 \\
\hline 0.063 & 12.04 & 1 & 150 & $\mathrm{NE}$ & 28 & 1 & 158 & $\mathrm{E}$ & 11 & 17.17 & 145.4 & $\mathrm{SE}$ & 2.4 & 55 \\
\hline 0.063 & 12.1 & 1 & 158 & $\mathrm{E}$ & 11 & 1 & 154 & $\mathrm{NE}$ & 25 & 14.05 & 151.2 & $\mathrm{SE}$ & $\begin{array}{l}.7 \\
1.3\end{array}$ & 241 \\
\hline 0.063 & 55.26 & 1 & 167 & $\mathrm{E}$ & 36 & 2 & 152.8 & $\mathrm{NE}$ & 35.3 & 8.3 & 63.7 & $\mathrm{NE}$ & 35.3 & 156 \\
\hline 0.063 & 55.32 & 2 & 152.8 & NE & 35.3 & 1 & 152 & $\mathrm{NE}$ & 33 & 2.31 & 161.4 & $\mathrm{~N}$ & 6.1 & 74 \\
\hline 0.063 & 55.38 & 1 & 152 & $\mathrm{NE}$ & 33 & 1 & 145 & $\mathrm{NE}$ & 37 & 5.66 & 109 & E & 23.9 & 194 \\
\hline 0.063 & 55.45 & 1 & 145 & $\mathrm{NE}$ & 37 & 1 & 172 & $\mathrm{E}$ & 26 & 17.66 & 110.2 & $\mathrm{E}$ & 23.3 & 14 \\
\hline 0.063 & 55.51 & 1 & 172 & E & 26 & 1 & 148 & $\mathrm{NE}$ & 32 & 12.98 & 100.1 & $\mathrm{E}$ & 24.9 & 188 \\
\hline 0.063 & 55.57 & 1 & 148 & NE & 32 & 1 & 155 & $\mathrm{NE}$ & 28 & 5.31 & 114.3 & $\mathrm{SE}$ & 19.1 & 20 \\
\hline 0.063 & 55.64 & 1 & 155 & $\mathrm{NE}$ & 28 & 1 & 156 & $\mathrm{NE}$ & 34 & 6.02 & 159.7 & $\mathrm{~N}$ & 2.5 & 250 \\
\hline 0.063 & 55.7 & 1 & 156 & $\mathrm{NE}$ & 34 & 1 & 175 & $E$ & 16 & 19.5 & 143 & SE & 8.6 & 50 \\
\hline 0.063 & 55.76 & 1 & 175 & $\mathrm{E}$ & 16 & 1 & 151 & $\mathrm{NE}$ & 23 & 10.51 & 115.3 & $\mathrm{SE}$ & 13.9 & 204 \\
\hline 0.063 & 55.83 & 1 & 151 & NE & 23 & 1 & 158 & $\mathrm{E}$ & 33 & 10.51 & 170.8 & $\mathrm{~N}$ & 8.2 & 262 \\
\hline 0.063 & 55.89 & 1 & 158 & $\mathrm{E}$ & 33 & 1 & 159 & $\mathrm{E}$ & 29 & 4.03 & 152.2 & $\mathrm{SE}$ & 3.8 & 61 \\
\hline 0.063 & 55.95 & 1 & 159 & $\mathrm{E}$ & 29 & 1 & 165 & $\mathrm{E}$ & 30 & 3.12 & 50.8 & $\mathrm{NE}$ & 27.8 & 323 \\
\hline 0.063 & 56.02 & 1 & 165 & $\mathrm{E}$ & 30 & 1 & 48 & NW & 8 & 34.31 & 176.1 & $\mathrm{~N}$ & 6.3 & 88 \\
\hline 0.063 & 56.08 & 1 & 48 & NW & 8 & 2 & 129.4 & $\mathrm{NE}$ & 28.9 & 28.7 & 144.1 & NW & 8 & 234 \\
\hline 0.063 & 56.14 & 2 & 129.4 & $\mathrm{NE}$ & 28.9 & 1 & 152 & $\mathrm{NE}$ & 53 & 27.98 & 166.5 & $\mathrm{~N}$ & 18.4 & 260 \\
\hline 0.063 & 56.2 & 1 & 152 & $\mathrm{NE}$ & 53 & 1 & 140 & $\mathrm{NE}$ & 52 & 9.57 & 46.2 & $\mathrm{NE}$ & 51.9 & 142 \\
\hline 0.063 & 56.27 & 1 & 140 & $\mathrm{NE}$ & 52 & 1 & 167 & $\mathrm{E}$ & 30 & 27.84 & 121.1 & $\mathrm{SE}$ & 22.5 & 21 \\
\hline 0.063 & 56.33 & 1 & 167 & $\mathrm{E}$ & 30 & 1 & 158 & $\mathrm{E}$ & 28 & 4.79 & 44.9 & $\mathrm{NE}$ & 26.1 & 138 \\
\hline 0.079 & 0.07 & 1 & 2 & $\mathrm{~s}$ & 40 & 1 & 173 & $\mathrm{E}$ & 37 & 6.35 & 53.2 & $\mathrm{NE}$ & 33.2 & 150 \\
\hline 0.079 & 0.47 & 1 & 159 & $\mathrm{E}$ & 29 & 1 & 170 & $\mathrm{E}$ & 37 & 9.97 & 16.8 & $\mathrm{~N}$ & 18.8 & 291 \\
\hline 0.079 & 0.71 & 1 & 162 & $\mathrm{E}$ & 30 & 1 & 168 & $\mathrm{E}$ & 37 & 7.74 & 6.6 & $\mathrm{~N}$ & 13.5 & 280 \\
\hline 0.079 & 11.35 & 1 & 152 & NE & 25 & 1 & 178 & $\mathrm{E}$ & 33 & 14.77 & 39.6 & $\mathrm{NE}$ & 23.3 & 312 \\
\hline 0.079 & 11.43 & 1 & 178 & $\mathrm{E}$ & 33 & 1 & 180 & $\mathrm{E}$ & 22 & 11.04 & 174.7 & $\mathrm{~s}$ & 2.1 & 84 \\
\hline 0.079 & 11.51 & 1 & 180 & $\mathrm{E}$ & 22 & 1 & 163 & $\mathrm{E}$ & 41 & 20.81 & 149.3 & $\mathrm{SE}$ & $\begin{array}{l}2.1 \\
11.7\end{array}$ & 237 \\
\hline 0.079 & 11.59 & 1 & 163 & E & 41 & 1 & 162 & $\mathrm{E}$ & 32 & 9.02 & 165.6 & $\mathrm{~N}$ & 2.2 & 76 \\
\hline 0.079 & 11.67 & 1 & 162 & $\mathrm{E}$ & 32 & 1 & 177 & $\mathrm{E}$ & 53 & 23.2 & 9.6 & $\mathrm{~N}$ & 16.1 & 284 \\
\hline 0.079 & 11.75 & 1 & 177 & $\mathrm{E}$ & 53 & 1 & 8 & $\mathrm{~s}$ & 29 & 24.98 & 169.3 & $\mathrm{~s}$ & 10.1 & 74 \\
\hline 0.079 & 11.83 & 1 & 8 & $\mathrm{~s}$ & 29 & 1 & 180 & $\mathrm{E}$ & 42 & 13.78 & 167.6 & $\mathrm{~s}$ & 10.9 & 255 \\
\hline 0.079 & 11.91 & 1 & 180 & $\mathrm{E}$ & 42 & 1 & 150 & $\mathrm{NE}$ & 28 & 21.84 & 31.1 & $\mathrm{NE}$ & 25 & 129 \\
\hline 0.079 & 11.99 & 1 & 150 & $\mathrm{NE}$ & 28 & 1 & 158 & E & 11 & $\begin{array}{l}17.17\end{array}$ & 145.4 & $\mathrm{SE}$ & 2.4 & 55 \\
\hline 0.079 & 12.22 & 1 & 154 & $\mathrm{NE}$ & 25 & 1 & 1 & W & 9 & 33.25 & 160.8 & $\mathrm{~N}$ & 3.1 & 71 \\
\hline 0.079 & 43.28 & 1 & 148 & $\mathrm{NE}$ & 19 & 1 & 163 & $\mathrm{E}$ & 26 & 9 & 12.9 & $\mathrm{~N}$ & 13.7 & 284 \\
\hline
\end{tabular}




\begin{tabular}{|c|c|c|c|c|c|c|c|c|c|c|c|c|c|c|}
\hline 0.1 & 12.09 & 1 & 158 & $\mathrm{E}$ & 11 & 1 & 154 & $\mathrm{NE}$ & 25 & 14.05 & 151.2 & $\mathrm{SE}$ & 1.3 & 241 \\
\hline 0.1 & 12.19 & 1 & 154 & $\mathrm{NE}$ & 25 & 1 & 1 & W & 9 & 33.25 & 160.8 & $\mathrm{~N}$ & 3.1 & 71 \\
\hline 0.1 & 23.49 & 1 & 145 & $\mathrm{NE}$ & 39 & 1 & 142 & $\mathrm{NE}$ & 24 & 15.08 & 148.7 & NW & 3 & 60 \\
\hline 0.1 & 24.09 & 1 & 140 & $\mathrm{NE}$ & 22 & 1 & 148 & $\mathrm{NE}$ & 25 & 4.37 & 8.3 & $N$ & 16.8 & 280 \\
\hline 0.1 & 42.19 & 1 & 156 & $\mathrm{NE}$ & 18 & 1 & 152 & $\mathrm{NE}$ & 22 & 4.23 & 136.2 & $\mathrm{SE}$ & 6.3 & 225 \\
\hline 0.1 & 42.99 & 1 & 154 & $\mathrm{NE}$ & 24 & 1 & 153 & $\mathrm{NE}$ & 22 & 2.04 & 163.7 & $N$ & 4.3 & 75 \\
\hline 0.1 & 43.29 & 1 & 148 & $\mathrm{NE}$ & 19 & 1 & 163 & $\mathrm{E}$ & 26 & 9 & 12.9 & $\mathrm{~N}$ & 13.7 & 284 \\
\hline 0.1 & 55.19 & 1 & 170 & $\mathrm{E}$ & 32 & 2 & 163.5 & $\mathrm{E}$ & 36.4 & 5.77 & 132.3 & SE & 20.9 & 218 \\
\hline 0.1 & 55.29 & 2 & 163.5 & $\mathrm{E}$ & 36.4 & 2 & 148.5 & $\mathrm{NE}$ & 33.5 & 9.08 & 43 & $\mathrm{NE}$ & 32.5 & 139 \\
\hline 0.1 & 55.39 & 2 & 148.5 & $\mathrm{NE}$ & 33.5 & 2 & 156.3 & $\mathrm{NE}$ & 30.8 & 4.95 & 99 & $E$ & 26.7 & 5 \\
\hline 0.1 & 55.49 & 2 & 156.3 & $\mathrm{NE}$ & 30.8 & 1 & 148 & $\mathrm{NE}$ & 32 & 4.5 & 80 & $E$ & 30.1 & 168 \\
\hline 0.1 & 55.59 & 1 & 148 & $\mathrm{NE}$ & 32 & 2 & 155.5 & $\mathrm{NE}$ & 31 & 4.06 & 78.3 & $E$ & 30.4 & 345 \\
\hline 0.1 & 55.69 & 2 & 155.5 & $\mathrm{NE}$ & 31 & 2 & 160.9 & $\mathrm{E}$ & 19.1 & 12.09 & 148.3 & SE & 4.3 & 57 \\
\hline 0.1 & 55.79 & 2 & 160.9 & $\mathrm{E}$ & 19.1 & 1 & 158 & $\mathrm{E}$ & 33 & 13.94 & 154.7 & $\mathrm{SE}$ & 2.1 & 244 \\
\hline 0.1 & 55.89 & 1 & 158 & $\mathrm{E}$ & 33 & 2 & 162 & E & 29.5 & 4.11 & 133.1 & $\mathrm{SE}$ & 15.3 & 39 \\
\hline 0.1 & 55.99 & 2 & 162 & $\mathrm{E}$ & 29.5 & 2 & 100.4 & $\mathrm{~N}$ & 15.1 & 25.64 & 10.5 & $N$ & 15.1 & 104 \\
\hline 0.1 & 56.09 & 2 & 100.4 & $\mathrm{~N}$ & 15.1 & 2 & 147.5 & $\mathrm{NE}$ & 42.9 & 34.1 & 162.4 & $\mathrm{~N}$ & 13.4 & 253 \\
\hline 0.1 & 56.19 & 2 & 147.5 & $\mathrm{NE}$ & 42.9 & 1 & 140 & $\mathrm{NE}$ & 52 & 10.67 & 121.3 & $\mathrm{SE}$ & 22.3 & 204 \\
\hline 0.1 & 56.29 & 1 & 140 & $\mathrm{NE}$ & 52 & 2 & 162.6 & $\mathrm{E}$ & 28.9 & 27.05 & 124.6 & $\mathrm{SE}$ & 18.8 & 26 \\
\hline 0.126 & 0.12 & 1 & 2 & $\mathrm{~s}$ & 40 & 1 & 173 & $\mathrm{E}$ & 37 & 6.35 & 53.2 & $\mathrm{NE}$ & 33.2 & 150 \\
\hline 0.126 & 0.24 & 1 & 173 & $\mathrm{E}$ & 37 & 1 & 166 & $\mathrm{E}$ & 29 & 8.85 & 11.4 & $\mathrm{~N}$ & 13.4 & 106 \\
\hline 0.126 & 0.37 & 1 & 166 & $\mathrm{E}$ & 29 & 1 & 159 & E & 29 & 3.39 & 72.5 & $\mathrm{E}$ & 29 & 163 \\
\hline 0.126 & 0.49 & 1 & 159 & $\mathrm{E}$ & 29 & 1 & 170 & $\mathrm{E}$ & 37 & 9.97 & 16.8 & $\mathrm{~N}$ & 18.8 & 291 \\
\hline 0.126 & 0.62 & 1 & 170 & $\mathrm{E}$ & 37 & 1 & 162 & $\mathrm{E}$ & 30 & 8.26 & 13.8 & $\mathrm{~N}$ & 16.9 & 109 \\
\hline 0.126 & 0.75 & 1 & 162 & $\mathrm{E}$ & 30 & 1 & 168 & $\mathrm{E}$ & 37 & 7.74 & 6.6 & $\mathrm{~N}$ & 13.5 & 280 \\
\hline 0.126 & 11.32 & 1 & 152 & $\mathrm{NE}$ & 25 & 1 & 178 & $\mathrm{E}$ & 33 & 14.77 & 39.6 & $\mathrm{NE}$ & 23.3 & 312 \\
\hline 0.126 & 11.45 & 1 & 178 & $\mathrm{E}$ & 33 & 2 & 169.2 & E & 31.2 & 5.01 & 59.8 & $\mathrm{NE}$ & 29.8 & 153 \\
\hline 0.126 & 11.57 & 2 & 169.2 & $\mathrm{E}$ & 31.2 & 1 & 162 & $\mathrm{E}$ & 32 & 3.83 & 88.9 & $\mathrm{E}$ & 30.9 & 178 \\
\hline 0.126 & 11.7 & 1 & 162 & $\mathrm{E}$ & 32 & 2 & 1.2 & $\mathrm{E}$ & 40.9 & 14.34 & 37.8 & $\mathrm{NE}$ & 27.3 & 312 \\
\hline 0.126 & 11.82 & 2 & 1.2 & $\mathrm{E}$ & 40.9 & 1 & 180 & $\mathrm{E}$ & 42 & 1.36 & 153.6 & $\mathrm{SE}$ & 21.8 & 235 \\
\hline 0.126 & 11.95 & 1 & 180 & $E$ & 42 & 2 & 152.3 & $\mathrm{NE}$ & 19.5 & 26.09 & 15.6 & $\mathrm{~N}$ & 13.6 & 111 \\
\hline 0.126 & 12.08 & 2 & 152.3 & $\mathrm{NE}$ & 19.5 & 1 & 154 & $\mathrm{NE}$ & 25 & 5.57 & 159.3 & $\mathrm{~N}$ & 2.5 & 250 \\
\hline 0.126 & 12.2 & 1 & 154 & $\mathrm{NE}$ & 25 & 1 & 1 & w & 9 & 33.25 & 160.8 & $\mathrm{~N}$ & 3.1 & 71 \\
\hline 0.126 & 12.33 & 1 & 1 & W & 9 & 1 & 176 & $\mathrm{E}$ & 47 & 55.97 & 176.6 & $\mathrm{~N}$ & 0.7 & 267 \\
\hline 0.126 & 13.21 & 1 & 176 & $\mathrm{E}$ & 47 & 1 & 13 & $\mathrm{~s}$ & 73 & 29.75 & 20.9 & $\mathrm{~N}$ & 24.3 & 300 \\
\hline 0.126 & 24.04 & 1 & 140 & $\mathrm{NE}$ & 22 & 1 & 148 & $\mathrm{NE}$ & 25 & 4.37 & 8.3 & $\mathrm{~N}$ & 16.8 & 280 \\
\hline 0.126 & 40.02 & 1 & 165 & $\mathrm{E}$ & 23 & 1 & 166 & $\mathrm{E}$ & 25 & 2.04 & 176 & $\mathrm{~N}$ & 4.6 & 267 \\
\hline 0.126 & 42.16 & 1 & 156 & $\mathrm{NE}$ & 18 & 1 & 152 & $\mathrm{NE}$ & 22 & 4.23 & 136.2 & $\mathrm{SE}$ & 6.3 & 225 \\
\hline 0.126 & 42.79 & 1 & 151 & $\mathrm{NE}$ & 24 & 1 & 154 & $\mathrm{NE}$ & 24 & 1.22 & 62.5 & $\mathrm{NE}$ & 24 & 332 \\
\hline 0.126 & 43.17 & 1 & 153 & $\mathrm{NE}$ & 22 & 1 & 148 & $\mathrm{NE}$ & 19 & 3.47 & 179.2 & $\mathrm{~N}$ & 10.1 & 91 \\
\hline 0.126 & 43.3 & 1 & 148 & $\mathrm{NE}$ & 19 & 1 & 163 & $\mathrm{E}$ & 26 & 9 & 12.9 & $\mathrm{~N}$ & 13.7 & 284 \\
\hline 0.126 & 55.13 & 1 & 170 & $\mathrm{E}$ & 32 & 1 & 167 & $\mathrm{E}$ & 36 & 4.34 & 149.3 & SE & 12.5 & 236 \\
\hline 0.126 & 55.26 & 1 & 167 & $\mathrm{E}$ & 36 & 3 & 152.5 & $\mathrm{NE}$ & 34.5 & 8.47 & 57.5 & $\mathrm{NE}$ & 34.4 & 151 \\
\hline 0.126 & 55.38 & 3 & 152.5 & $\mathrm{NE}$ & 34.5 & 2 & 156.3 & $\mathrm{NE}$ & 30.8 & 4.23 & 129.3 & $\mathrm{SE}$ & 15.2 & 35 \\
\hline 0.126 & 55.51 & 2 & 156.3 & $\mathrm{NE}$ & 30.8 & 2 & 151.3 & $\mathrm{NE}$ & 30 & 2.69 & 42.7 & $\mathrm{NE}$ & 28.6 & 137 \\
\hline 0.126 & 55.63 & 2 & 151.3 & $\mathrm{NE}$ & 30 & 2 & 162.2 & $\mathrm{E}$ & 24.7 & 7.23 & 116.1 & SE & 18.4 & 23 \\
\hline 0.126 & 55.76 & 2 & 162.2 & $\mathrm{E}$ & 24.7 & 2 & 155.1 & $\mathrm{NE}$ & 28 & 4.52 & 117.1 & $\mathrm{SE}$ & 18.1 & 205 \\
\hline 0.126 & 55.89 & 2 & 155.1 & $\mathrm{NE}$ & 28 & 2 & 162 & $\mathrm{E}$ & 29.5 & 3.67 & 41.4 & $\mathrm{NE}$ & 25.9 & 314 \\
\hline 0.126 & 56.01 & 2 & 162 & $\mathrm{E}$ & 29.5 & 3 & 121.5 & $\mathrm{NE}$ & 20.1 & 18.9 & 21.7 & $N$ & 19.8 & 116 \\
\hline 0.126 & 56.14 & 3 & 121.5 & $\mathrm{NE}$ & 20.1 & 2 & 146 & $\mathrm{NE}$ & 52.3 & 34.8 & 155 & NW & 11.4 & 247 \\
\hline 0.126 & 56.26 & 2 & 146 & $\mathrm{NE}$ & 52.3 & 2 & 162.6 & $\mathrm{E}$ & 28.9 & 25.63 & 134.4 & SE & 14.7 & 37 \\
\hline 0.158 & 0.14 & 2 & 177.6 & $\mathrm{E}$ & 38.4 & 1 & 166 & $\mathrm{E}$ & 29 & 11.38 & 21.8 & $N$ & 17.9 & 117 \\
\hline 0.158 & 0.3 & 1 & 166 & $\mathrm{E}$ & 29 & 1 & 159 & $\mathrm{E}$ & 29 & 3.39 & 72.5 & E & 29 & 163 \\
\hline 0.158 & 0.45 & 1 & 159 & $\mathrm{E}$ & 29 & 1 & 170 & $\mathrm{E}$ & 37 & 9.97 & 16.8 & $N$ & 18.8 & 291 \\
\hline 0.158 & 0.61 & 1 & 170 & $\mathrm{E}$ & 37 & 2 & 165.3 & $\mathrm{E}$ & 33.5 & 4.46 & 19.9 & $\mathrm{~N}$ & 20.6 & 116 \\
\hline 0.158 & 11.39 & 2 & 166.7 & $\mathrm{E}$ & 28.4 & 1 & 180 & $\mathrm{E}$ & 22 & 8.51 & 134.3 & SE & 16.1 & 41 \\
\hline 0.158 & 11.55 & 1 & 180 & $\mathrm{E}$ & 22 & 2 & 162.6 & $\mathrm{E}$ & 36.5 & 16.68 & 143.7 & SE & 13.5 & 232 \\
\hline 0.158 & 11.71 & 2 & 162.6 & $\mathrm{E}$ & 36.5 & 2 & 1.2 & $\mathrm{E}$ & 40.9 & 12.38 & 56.3 & $\mathrm{NE}$ & 35.4 & 329 \\
\hline 0.158 & 11.87 & 2 & 1.2 & $\mathrm{E}$ & 40.9 & 2 & 167.7 & $E$ & 34.1 & 10.6 & 38.4 & $\mathrm{NE}$ & 27.7 & 136 \\
\hline 0.158 & 12.02 & 2 & 167.7 & $\mathrm{E}$ & 34.1 & 2 & 155.2 & $\mathrm{NE}$ & 18 & 16.93 & 178.7 & $\mathrm{~N}$ & 7.4 & 91 \\
\hline 0.158 & 12.18 & 2 & 155.2 & $\mathrm{NE}$ & 18 & 1 & 1 & w & 9 & 26.38 & 163.6 & $\mathrm{~N}$ & 2.7 & 74 \\
\hline 0.158 & 12.34 & 1 & 1 & W & 9 & 1 & 176 & $\mathrm{E}$ & 47 & 55.97 & 176.6 & $N$ & 0.7 & 267 \\
\hline 0.158 & 13.29 & 1 & 176 & $\mathrm{E}$ & 47 & 1 & 13 & $\mathrm{~s}$ & 73 & 29.75 & 20.9 & $\mathrm{~N}$ & 24.3 & 300 \\
\hline 0.158 & 14.72 & 1 & 141 & $\mathrm{NE}$ & 14 & 1 & 164 & $\mathrm{E}$ & 29 & 16.94 & 0.7 & $\mathrm{~N}$ & 9 & 272 \\
\hline 0.158 & 23.44 & 1 & 145 & $\mathrm{NE}$ & 39 & 1 & 142 & $\mathrm{NE}$ & 24 & 15.08 & 148.7 & NW & 3 & 60 \\
\hline 0.158 & 24.07 & 1 & 140 & $\mathrm{NE}$ & 22 & 1 & 148 & $\mathrm{NE}$ & 25 & 4.37 & 8.3 & $\mathrm{~N}$ & 16.8 & 280 \\
\hline 0.158 & 39.6 & 1 & 148 & $\mathrm{NE}$ & 30 & 1 & 133 & $\mathrm{NE}$ & 23 & 9.64 & 1.3 & $\mathrm{~N}$ & 17.6 & 95 \\
\hline 0.158 & 40.08 & 1 & 165 & $\mathrm{E}$ & 23 & 1 & 166 & $\mathrm{E}$ & 25 & 2.04 & 176 & $\mathrm{~N}$ & 4.6 & 267 \\
\hline 0.158 & 42.14 & 1 & 156 & $\mathrm{NE}$ & 18 & 1 & 152 & $\mathrm{NE}$ & 22 & 4.23 & 136.2 & SE & 6.3 & 225 \\
\hline 0.158 & 42.3 & 1 & 152 & $\mathrm{NE}$ & 22 & 1 & 153 & $\mathrm{NE}$ & 26 & 4.02 & 157.8 & $\mathrm{~N}$ & 2.3 & 248 \\
\hline 0.158 & 42.77 & 1 & 151 & $\mathrm{NE}$ & 24 & 1 & 154 & $\mathrm{NE}$ & 24 & 1.22 & 62.5 & $\mathrm{NE}$ & 24 & 332 \\
\hline 0.158 & 42.93 & 1 & 154 & $\mathrm{NE}$ & 24 & 1 & 153 & $\mathrm{NE}$ & 22 & 2.04 & 163.7 & $N$ & 4.3 & 75 \\
\hline 0.158 & 43.09 & 1 & 153 & $\mathrm{NE}$ & 22 & 1 & 148 & $\mathrm{NE}$ & 19 & 3.47 & 179.2 & $\mathrm{~N}$ & 10.1 & 91 \\
\hline 0.158 & 43.25 & 1 & 148 & $\mathrm{NE}$ & 19 & 1 & 163 & $\mathrm{E}$ & 26 & 9 & 12.9 & $N$ & 13.7 & 284 \\
\hline 0.158 & 55.13 & 1 & 170 & $\mathrm{E}$ & 32 & 2 & 163.5 & $\mathrm{E}$ & 36.4 & 5.77 & 132.3 & SE & 20.9 & 218 \\
\hline 0.158 & 55.29 & 2 & 163.5 & $\mathrm{E}$ & 36.4 & 3 & 147.2 & $\mathrm{NE}$ & 34.6 & 9.58 & 52.1 & $\mathrm{NE}$ & 34.5 & 146 \\
\hline 0.158 & 55.45 & 3 & 147.2 & $\mathrm{NE}$ & 34.6 & 3 & 157.6 & $\mathrm{E}$ & 28.3 & 8.29 & 116.2 & $\mathrm{SE}$ & 19.6 & 21 \\
\hline 0.158 & 55.61 & 3 & 157.6 & $\mathrm{E}$ & 28.3 & 2 & 162.2 & $\mathrm{E}$ & 24.7 & 4.13 & 132.2 & SE & 13 & 40 \\
\hline 0.158 & 55.77 & 2 & 162.2 & E & 24.7 & 3 & 156.4 & $\mathrm{NE}$ & 28.3 & 4.41 & 126.2 & SE & 15.2 & 214 \\
\hline
\end{tabular}




\begin{tabular}{|c|c|c|c|c|c|c|c|c|c|c|c|c|c|c|}
\hline 0.2 & 43.25 & 1 & 148 & $\mathrm{NE}$ & 19 & 1 & 163 & $\mathrm{E}$ & 26 & 9 & 12.9 & $\mathrm{~N}$ & 13.7 & 284 \\
\hline 0.2 & 43.85 & 1 & 152 & $\mathrm{NE}$ & 22 & 1 & 147 & NE & 23 & 2.16 & 89 & $\mathrm{E}$ & 19.8 & 177 \\
\hline 0.2 & 55.23 & 2 & 168.4 & $\mathrm{E}$ & 34 & 3 & 152.5 & $\mathrm{NE}$ & 34.5 & 8.94 & 74.4 & $\mathrm{E}$ & 33.9 & 165 \\
\hline 0.2 & 55.43 & 3 & 152.5 & $\mathrm{NE}$ & 34.5 & 4 & 153.8 & $\mathrm{NE}$ & 30.4 & 4.21 & 145.2 & $\mathrm{SE}$ & 5 & 53 \\
\hline 0.2 & 55.62 & 4 & 153.8 & $\mathrm{NE}$ & 30.4 & 3 & 158.6 & $\mathrm{E}$ & 24.1 & 6.67 & 139 & $\mathrm{SE}$ & 8.5 & 47 \\
\hline 0.2 & 55.82 & 3 & 158.6 & $\mathrm{E}$ & 24.1 & 3 & 160.6 & $\mathrm{E}$ & 30.6 & 6.64 & 166.7 & $\mathrm{~N}$ & 3.6 & 258 \\
\hline 0.2 & 56.02 & 3 & 160.6 & $\mathrm{E}$ & 30.6 & 4 & 134.7 & NE & 27.5 & 12.85 & 42 & $\mathrm{NE}$ & 27.5 & 136 \\
\hline 0.2 & 56.22 & 4 & 134.7 & $\mathrm{NE}$ & 27.5 & 3 & 152.7 & NE & 35.8 & 12.51 & 8 & $\mathrm{~N}$ & 22.6 & 281 \\
\hline 0.251 & 0.18 & 2 & 177.6 & $\mathrm{E}$ & 38.4 & 2 & 162.5 & $\mathrm{E}$ & 29 & 12.59 & 26.8 & $\mathrm{NE}$ & 21.1 & 123 \\
\hline 0.251 & 0.43 & 2 & 162.5 & $\mathrm{E}$ & 29 & 2 & 166.4 & $E$ & 33.4 & 4.91 & 5.4 & $\mathrm{~N}$ & 12.1 & 279 \\
\hline 0.251 & 0.68 & 2 & 166.4 & $\mathrm{E}$ & 33.4 & 1 & 168 & $\mathrm{E}$ & $\begin{array}{c}5.4 \\
37\end{array}$ & 3.69 & $\begin{array}{c}17.4 \\
179.4\end{array}$ & $\mathrm{~N}$ & 8.4 & 271 \\
\hline 0.251 & 0.94 & 1 & 168 & $\mathrm{E}$ & 37 & 1 & 151 & NE & 28 & 12.75 & 20.4 & $\mathrm{~N}$ & 22 & 116 \\
\hline 0.251 & 1.19 & 1 & 151 & $\mathrm{NE}$ & 28 & 1 & 155 & $\mathrm{NE}$ & 39 & 11.21 & 162.6 & $\mathrm{~N}$ & 6.1 & 254 \\
\hline 0.251 & 1.94 & 1 & 156 & $\mathrm{NE}$ & 48 & 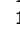 & 156 & $\mathrm{NE}$ & 29 & 19 & 156 & NW & 0 & 66 \\
\hline 0.251 & 11.49 & 3 & 170.5 & E & 26.1 & 3 & 168.3 & $E$ & 41.7 & 15.67 & 165.7 & $\mathrm{~s}$ & 2.3 & 255 \\
\hline 0.251 & 11.74 & 3 & 168.3 & $\mathrm{E}$ & 41.7 & 3 & 173.9 & $\mathrm{E}$ & 32 & 10.27 & 155.4 & $\mathrm{SE}$ & 11.2 & 61 \\
\hline 0.251 & 11.99 & 3 & 173.9 & $\mathrm{E}$ & 32 & 3 & 147.3 & NE & 9.5 & 23.89 & 2.9 & $\mathrm{~N}$ & 5.6 & 94 \\
\hline 0.251 & 12.24 & 3 & 147.3 & $\mathrm{NE}$ & 9.5 & 1 & 176 & $\mathrm{E}$ & 47 & 38.87 & 1 & $\mathrm{~N}$ & 5.3 & 271 \\
\hline 0.251 & 12.49 & 1 & 176 & $\mathrm{E}$ & 47 & 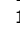 & 169 & $\mathrm{E}$ & 49 & 5.57 & 112.3 & $\mathrm{E}$ & 43.9 & 195 \\
\hline 0.251 & 13.24 & 1 & 176 & $\mathrm{E}$ & 47 & 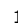 & 13 & $\mathrm{~s}$ & 73 & 29.75 & 20.9 & $\mathrm{~N}$ & 24.3 & 300 \\
\hline 0.251 & 13.5 & 1 & 13 & $\mathrm{~S}$ & 73 & 1 & 179 & $\mathrm{E}$ & 74 & 13.46 & 110.6 & $\mathrm{E}$ & 72.9 & 195 \\
\hline 0.251 & 13.75 & 1 & 179 & $\mathrm{E}$ & 74 & 1 & 177 & $\mathrm{E}$ & 58 & 16.1 & 0.7 & $\mathrm{~N}$ & 5.9 & 95 \\
\hline 0.251 & 14.5 & 1 & 180 & $\mathrm{E}$ & 60 & 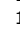 & 141 & NE & 14 & 49.61 & 5.8 & $\mathrm{~N}$ & 10 & 102 \\
\hline 0.251 & 14.75 & 1 & 141 & $\mathrm{NE}$ & 14 & 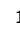 & 164 & $E$ & 29 & 16.94 & 0.7 & $\mathrm{~N}$ & 9 & 272 \\
\hline 0.251 & 23.29 & 1 & 155 & $\mathrm{NE}$ & 44 & 2 & 143.8 & NE & 31.5 & 14.22 & 173 & $\mathrm{~N}$ & 16.6 & 89 \\
\hline 0.251 & 24.05 & 1 & 140 & $\mathrm{NE}$ & 22 & 1 & 148 & $\mathrm{NE}$ & 25 & 4.37 & 8.3 & $\mathrm{~N}$ & 16.8 & 280 \\
\hline 0.251 & 24.3 & 1 & 148 & $\mathrm{NE}$ & 25 & 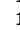 & 155 & $\mathrm{NE}$ & 22 & 4.09 & 111 & $\mathrm{E}$ & 15.7 & 18 \\
\hline 0.251 & 24.55 & 1 & 155 & $\mathrm{NE}$ & 22 & 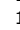 & 153 & $\mathrm{NE}$ & 39 & 17.03 & 151 & SE & 1.6 & 241 \\
\hline 0.251 & 39.12 & 1 & 160 & E & 25 & 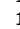 & 157 & NE & 33 & 8.13 & 149.4 & $\mathrm{SE}$ & 4.9 & 238 \\
\hline 0.251 & 39.37 & 1 & 157 & $\mathrm{NE}$ & 33 & 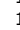 & 148 & $\mathrm{NE}$ & 30 & 5.57 & 25.8 & $\mathrm{NE}$ & 26 & 121 \\
\hline 0.251 & 39.62 & 1 & 148 & $\mathrm{NE}$ & 30 & 1 & 133 & $\mathrm{NE}$ & 23 & 9.64 & 1.3 & $\mathrm{~N}$ & 17.6 & 95 \\
\hline 0.251 & 39.87 & 1 & 133 & $\mathrm{NE}$ & 23 & 2 & 165.5 & $\mathrm{E}$ & 24 & 12.86 & 54.6 & NE & 22.6 & 324 \\
\hline 0.251 & 41.13 & 1 & 150 & $\mathrm{NE}$ & 23 & 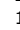 & 151 & NE & 19 & 4.02 & 145.7 & $\mathrm{SE}$ & 1.8 & 55 \\
\hline 0.251 & 41.88 & 1 & 155 & $\mathrm{NE}$ & 26 & 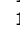 & 156 & NE & 18 & 8.01 & 153 & $\mathrm{SE}$ & 1 & 63 \\
\hline 0.251 & 42.13 & 1 & 156 & $\mathrm{NE}$ & 18 & 1 & 152 & NE & 22 & 4.23 & 136.2 & SE & 6.3 & 225 \\
\hline 0.251 & 42.38 & 1 & 152 & $\mathrm{NE}$ & 22 & 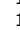 & 153 & NE & 26 & 4.02 & 157.8 & $\mathrm{~N}$ & 2.3 & 248 \\
\hline 0.251 & 42.63 & 1 & 153 & $\mathrm{NE}$ & 26 & 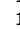 & 151 & NE & 24 & 2.17 & 173 & $\mathrm{~N}$ & 9.5 & 85 \\
\hline 0.251 & 42.88 & 1 & 151 & $\mathrm{NE}$ & 24 & 2 & 153.5 & $\mathrm{NE}$ & 23 & 1.42 & 109.6 & $\mathrm{E}$ & 16.4 & 17 \\
\hline 0.251 & 43.14 & 2 & 153.5 & $\mathrm{NE}$ & 23 & 2 & 156.6 & $\mathrm{NE}$ & 22.3 & 1.37 & 96.4 & $\mathrm{E}$ & 19.6 & 5 \\
\hline 0.251 & $\begin{array}{r}43.89 \\
43.8\end{array}$ & 1 & 152 & $\mathrm{NE}$ & 22 & 1 & 147 & $\mathrm{NE}$ & 23 & 2.16 & $\begin{array}{c}8.4 \\
89\end{array}$ & $\mathrm{E}$ & 19.8 & 177 \\
\hline 0.251 & 55.19 & 1 & 170 & $\mathrm{E}$ & 32 & 5 & 153.9 & $\mathrm{NE}$ & 35.1 & 9.4 & 94.4 & $\mathrm{E}$ & 31.2 & 182 \\
\hline 0.251 & 55.44 & 5 & 153.9 & $\mathrm{NE}$ & 35.1 & 2 & 157.1 & $\mathrm{NE}$ & 29.7 & 5.63 & 140.2 & $\mathrm{SE}$ & 9.4 & 48 \\
\hline 0.251 & 55.7 & 4 & 157.1 & $\mathrm{NE}$ & 29.7 & 4 & 159.4 & $\mathrm{E}$ & 25.1 & 4.76 & 146.9 & $\mathrm{SE}$ & 5.8 & 55 \\
\hline 0.251 & 55.95 & 4 & 159.4 & $\mathrm{E}$ & 25.1 & 5 & 141.7 & $\mathrm{NE}$ & 27.6 & 8.18 & 80 & $\mathrm{E}$ & 24.7 & 169 \\
\hline 0.251 & 56.2 & 5 & 141.7 & $\mathrm{NE}$ & 27.6 & 3 & 152.7 & $\mathrm{NE}$ & 35.8 & 10.05 & 178.1 & $\mathrm{~N}$ & 17.2 & 272 \\
\hline 0.316 & 0.2 & 2 & 177.6 & $\mathrm{E}$ & 38.4 & 3 & 165.4 & $\mathrm{E}$ & 31.6 & 9.79 & 31.8 & NE & 24 & 129 \\
\hline 0.316 & 0.52 & 3 & 165.4 & $\mathrm{E}$ & 31.6 & 2 & 165.3 & $\mathrm{E}$ & 33.5 & 1.89 & 164.1 & $\mathrm{~s}$ & 0.8 & 254 \\
\hline 0.316 & 0.84 & 2 & 165.3 & $\mathrm{E}$ & 33.5 & 1 & 151 & NE & 28 & 9.08 & 27.3 & NE & 23.9 & 122 \\
\hline 0.316 & 1.15 & 1 & 151 & $\mathrm{NE}$ & 28 & 1 & 155 & $\mathrm{NE}$ & 39 & 11.21 & 162.6 & $\mathrm{~N}$ & 6.1 & 254 \\
\hline 0.316 & 2.1 & 1 & 156 & $\mathrm{NE}$ & 48 & 1 & 156 & $\mathrm{NE}$ & 29 & 19 & 156 & NW & 0 & 66 \\
\hline 0.316 & 5.58 & 1 & 178 & E & 28 & 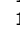 & 150 & $\mathrm{NE}$ & 30 & 13.61 & 83.4 & E & 27.9 & 174 \\
\hline 0.316 & 11.59 & 4 & 168 & $\mathrm{E}$ & 29.8 & 4 & 176.8 & $\mathrm{E}$ & 38.6 & 10.12 & 17.3 & $\mathrm{~N}$ & 15.7 & 291 \\
\hline 0.316 & 11.9 & 4 & 176.8 & $\mathrm{E}$ & 38.6 & 3 & 153 & NE & 21.4 & 20.69 & 16.5 & $\mathrm{~N}$ & 15.1 & 112 \\
\hline 0.316 & 12.22 & 3 & 153 & $\mathrm{NE}$ & 21.4 & 2 & 174.6 & $\mathrm{E}$ & $\begin{array}{c}2.4 \\
19\end{array}$ & $\begin{array}{l}2.09 \\
7.79\end{array}$ & $\begin{array}{l}9.3 \\
92\end{array}$ & $\mathrm{E}$ & $\begin{array}{l}18.9 \\
18.9\end{array}$ & 1 \\
\hline 0.316 & 12.54 & 2 & 174.6 & $\mathrm{E}$ & 19 & 1 & 169 & $\mathrm{E}$ & 49 & 30.11 & 166.6 & $\mathrm{~s}$ & 2.8 & 256 \\
\hline 0.316 & 12.85 & 1 & 169 & $\mathrm{E}$ & 49 & 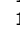 & 176 & $\mathrm{E}$ & 47 & 5.57 & 112.3 & $\mathrm{E}$ & 43.9 & 12 \\
\hline 0.316 & 13.17 & 1 & 176 & $\mathrm{E}$ & 47 & 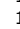 & 13 & $\mathrm{~s}$ & 73 & 29.75 & 20.9 & $\mathrm{~N}$ & 24.3 & 300 \\
\hline 0.316 & 13.49 & 1 & 13 & $\mathrm{~s}$ & 73 & 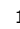 & 179 & $\mathrm{E}$ & 74 & 13.46 & 110.6 & $\mathrm{E}$ & 72.9 & 195 \\
\hline 0.316 & 13.8 & 1 & 179 & $\mathrm{E}$ & 74 & 1 & 177 & $\mathrm{E}$ & 58 & 16.1 & 0.7 & $\mathrm{~N}$ & 5.9 & 95 \\
\hline 0.316 & 14.12 & 1 & 177 & $\mathrm{E}$ & 58 & 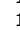 & 180 & $\mathrm{E}$ & 60 & 3.26 & 32 & $\mathrm{NE}$ & 42.6 & 320 \\
\hline 0.316 & 14.43 & 1 & 180 & $\mathrm{E}$ & 60 & 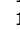 & 141 & $\mathrm{NE}$ & 14 & 49.61 & 5.8 & $\mathrm{~N}$ & 10 & 102 \\
\hline 0.316 & 14.75 & 1 & 141 & $\mathrm{NE}$ & 14 & 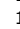 & 164 & $\mathrm{E}$ & 29 & 16.94 & 0.7 & $\mathrm{~N}$ & 9 & 272 \\
\hline 0.316 & 23.29 & 1 & 155 & $\mathrm{NE}$ & 44 & 2 & 143.8 & $\mathrm{NE}$ & 31.5 & 14.22 & 173 & $\mathrm{~N}$ & 16.6 & 89 \\
\hline 0.316 & 24.24 & 2 & 144.2 & $\mathrm{NE}$ & 23.4 & 1 & 155 & $\mathrm{NE}$ & 22 & 4.39 & 80.3 & $\mathrm{E}$ & 21.3 & 348 \\
\hline 0.316 & 24.55 & 1 & 155 & $\mathrm{NE}$ & 22 & 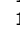 & 153 & $\mathrm{NE}$ & 39 & 17.03 & 151 & $\mathrm{SE}$ & 1.6 & 241 \\
\hline 0.316 & 24.87 & 1 & 153 & $\mathrm{NE}$ & 39 & 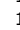 & 156 & $\mathrm{NE}$ & 30 & 9.16 & 145.6 & $\mathrm{SE}$ & 5.9 & 54 \\
\hline 0.316 & 39.1 & 1 & 160 & $\mathrm{E}$ & 25 & 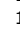 & 157 & $\mathrm{NE}$ & 33 & 8.13 & 149.4 & $\mathrm{SE}$ & 4.9 & 238 \\
\hline 0.316 & 39.42 & 1 & 157 & $\mathrm{NE}$ & 33 & 2 & 141.4 & $\mathrm{NE}$ & 26.3 & 10.16 & 14.5 & $\mathrm{~N}$ & 21.6 & 109 \\
\hline 0.316 & 39.73 & 2 & 141.4 & $\mathrm{NE}$ & 26.3 & 1 & 165 & $\mathrm{E}$ & 23 & 10.3 & 83.2 & $\mathrm{E}$ & 22.8 & 350 \\
\hline 0.316 & 40.05 & 1 & 165 & E & 23 & 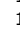 & 166 & $\mathrm{E}$ & 25 & 2.04 & 176 & $\mathrm{~N}$ & 4.6 & 267 \\
\hline 0.316 & 40.36 & 1 & 166 & $\mathrm{E}$ & 25 & 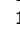 & 153 & $\mathrm{NE}$ & 24 & 5.47 & 58 & $\mathrm{NE}$ & 23.9 & 150 \\
\hline 0.316 & 40.68 & 1 & 153 & $\mathrm{NE}$ & 24 & 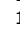 & 150 & $\mathrm{NE}$ & 23 & 1.56 & 19.2 & $\mathrm{~N}$ & 17.8 & 112 \\
\hline 0.316 & 41.63 & 1 & 151 & $\mathrm{NE}$ & 19 & 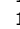 & 155 & $\mathrm{NE}$ & 26 & 7.16 & 164.5 & $\mathrm{~N}$ & 4.6 & 255 \\
\hline 0.316 & 41.95 & 1 & 155 & $\mathrm{NE}$ & 26 & 1 & 156 & $\mathrm{NE}$ & 18 & 8.01 & 153 & SE & $\begin{array}{l}4.0 \\
1\end{array}$ & 63 \\
\hline 0.316 & 42.26 & 1 & 156 & $\mathrm{NE}$ & 18 & 2 & 152.5 & $\mathrm{NE}$ & 24 & 6.12 & 143.3 & $\mathrm{SE}$ & 4.1 & 233 \\
\hline 0.316 & 42.58 & 2 & 152.5 & $\mathrm{NE}$ & 24 & 1 & 151 & $\mathrm{NE}$ & 24 & 0.63 & 61.9 & $\mathrm{NE}$ & 24 & 152 \\
\hline 0.316 & 42.89 & 1 & 151 & $\mathrm{NE}$ & 24 & 3 & 151.9 & $\mathrm{NE}$ & 21.6 & 2.38 & 143.7 & $\mathrm{SE}$ & 3.3 & 53 \\
\hline 0.316 & 43.21 & 3 & 151.9 & $\mathrm{NE}$ & 21.6 & 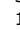 & 163 & $\mathrm{E}$ & 26 & 6.23 & 20.9 & $\mathrm{~N}$ & $\begin{array}{c}16.7 \\
\end{array}$ & 293 \\
\hline 0.316 & 43.53 & 1 & 163 & E & 26 & 1 & 152 & $\mathrm{NE}$ & 22 & 5.99 & 23.2 & $\mathrm{NE}$ & 17.5 & 116 \\
\hline
\end{tabular}




\begin{tabular}{|c|c|c|c|c|c|c|c|c|c|c|c|c|c|c|}
\hline 0.398 & 38.94 & 1 & 160 & $E$ & 25 & 1 & 157 & NE & 33 & 8.13 & 149.4 & SE & 4.9 & 238 \\
\hline 0.398 & 39.34 & 1 & 157 & NE & 33 & 2 & 141.4 & $\mathrm{NE}$ & 26.3 & 10.16 & 14.5 & $\mathrm{~N}$ & 21.6 & 109 \\
\hline 0.398 & 39.74 & 2 & 141.4 & NE & 26.3 & 2 & 165.5 & $\mathrm{E}$ & 24 & 10.43 & 77.2 & $E$ & 24 & 345 \\
\hline 0.398 & 40.13 & 2 & 165.5 & $\mathrm{E}$ & 24 & 1 & 153 & $\mathrm{NE}$ & 24 & 5.08 & 69.3 & $E$ & 23.9 & 160 \\
\hline 0.398 & 40.53 & 1 & 153 & NE & 24 & 1 & 150 & $\mathrm{NE}$ & 23 & 1.56 & 19.2 & $\mathrm{~N}$ & 17.8 & 112 \\
\hline 0.398 & 41.73 & 1 & 151 & $\mathrm{NE}$ & 19 & 2 & 155.4 & $\mathrm{NE}$ & 22 & 3.37 & 179 & $\mathrm{~N}$ & 9.2 & 270 \\
\hline 0.398 & 42.12 & 2 & 155.4 & $\mathrm{NE}$ & 22 & 2 & 152.5 & $\mathrm{NE}$ & 24 & 2.29 & 126.6 & SE & 11 & 215 \\
\hline 0.398 & 42.52 & 2 & 152.5 & $\mathrm{NE}$ & 24 & 2 & 152.5 & $\mathrm{NE}$ & 24 & 0.02 & 38.5 & $\mathrm{NE}$ & 22.1 & 153 \\
\hline 0.398 & 42.92 & 2 & 152.5 & NE & 24 & 2 & 150.7 & $\mathrm{NE}$ & 20.5 & 3.58 & 161.9 & $\mathrm{~N}$ & 4.2 & 73 \\
\hline 0.398 & 43.32 & 2 & 150.7 & NE & 20.5 & 2 & 157.9 & $\mathrm{E}$ & 23.9 & 4.38 & 10.9 & $\mathrm{~N}$ & 13.6 & 283 \\
\hline 0.398 & 43.72 & 2 & 157.9 & $\mathrm{E}$ & 23.9 & 1 & 147 & $\mathrm{NE}$ & 23 & 4.44 & 49.8 & $\mathrm{NE}$ & 22.8 & 141 \\
\hline 0.398 & 55.26 & 3 & 165.5 & $E$ & 34.9 & 6 & 151.9 & $\mathrm{NE}$ & 31.4 & 8.22 & 39.1 & $\mathrm{NE}$ & 29.3 & 135 \\
\hline 0.398 & 55.66 & 6 & 151.9 & NE & $=31.4$ & 7 & 157.3 & $\mathrm{NE}$ & 23.5 & 8.21 & 138.9 & SE & 7.8 & 47 \\
\hline 0.398 & 56.06 & 7 & 157.3 & NE & 23.5 & $\epsilon$ & 146.2 & $\mathrm{NE}$ & 36 & 13.54 & 130.5 & SE & 11.1 & 219 \\
\hline 0.501 & 0.37 & 3 & 174.4 & $\mathrm{E}$ & 35.2 & 4 & 165.2 & $\mathrm{E}$ & 33.2 & 5.53 & 54.8 & $\mathrm{NE}$ & 31.5 & 149 \\
\hline 0.501 & 0.88 & 4 & 165.2 & $\mathrm{E}$ & 33.2 & 1 & 151 & $\mathrm{NE}$ & 28 & 8.85 & 28.5 & $\mathrm{NE}$ & 24.2 & 123 \\
\hline 0.501 & 1.38 & 1 & 151 & NE & 28 & 2 & 155.5 & $\mathrm{NE}$ & 43.5 & 15.71 & 161.3 & $\mathrm{~N}$ & 5.4 & 253 \\
\hline 0.501 & 1.88 & 2 & 155.5 & NE & 43.5 & 1 & 156 & $\mathrm{NE}$ & 29 & 14.5 & 154.9 & SE & 0.6 & 65 \\
\hline 0.501 & 5.89 & 1 & 178 & $\mathrm{E}$ & 28 & 1 & 150 & $\mathrm{NE}$ & 30 & 13.61 & 83.4 & $\mathrm{E}$ & 27.9 & 174 \\
\hline 0.501 & 6.39 & 1 & 150 & $\mathrm{NE}$ & 30 & 1 & 149 & $\mathrm{NE}$ & 27 & 3.04 & 157.5 & NW & 4.3 & 69 \\
\hline 0.501 & 11.4 & 2 & 166.7 & $\mathrm{E}$ & 28.4 & 6 & 174.5 & $\mathrm{E}$ & 36.1 & 8.77 & 15.3 & $\mathrm{~N}$ & 14.5 & 288 \\
\hline 0.501 & 11.9 & 6 & 174.5 & $\mathrm{E}$ & 36.1 & 5 & 159.9 & $\mathrm{E}$ & 20.3 & 17.15 & 8.6 & $\mathrm{~N}$ & 10.1 & 102 \\
\hline 0.501 & 12.4 & 5 & 159.9 & $E$ & 20.3 & 1 & 169 & E & 49 & 29.1 & 173.2 & $\mathrm{~N}$ & 4.9 & 264 \\
\hline 0.501 & 12.9 & 1 & 169 & $\mathrm{E}$ & 49 & 2 & 5.6 & $\mathrm{E}$ & 59.7 & 17.21 & 34 & NE & 39.1 & 316 \\
\hline 0.501 & 13.4 & 2 & 5.6 & $\mathrm{E}$ & 59.7 & 1 & 179 & $\mathrm{E}$ & 74 & 15.51 & 172.7 & $\mathrm{~s}$ & 21 & 251 \\
\hline 0.501 & 13.91 & 1 & 179 & E & 74 & 2 & 178.5 & $\mathrm{E}$ & 59 & 15.02 & 179.4 & $\mathrm{~N}$ & 1.5 & 91 \\
\hline 0.501 & 14.41 & 2 & 178.5 & $\mathrm{E}$ & 59 & 2 & 156.4 & NE & 21.2 & 39.92 & 4.9 & $\mathrm{~N}$ & 10.5 & 101 \\
\hline 0.501 & 20.42 & 1 & 131 & NE & $=47$ & 1 & 145 & $\mathrm{NE}$ & 29 & 19.86 & 116.9 & SE & 14.6 & 21 \\
\hline 0.501 & 20.92 & 1 & 145 & NE & $=29$ & 1 & 179 & $\mathrm{E}$ & 15 & 18.41 & 120.7 & SE & 12.8 & 28 \\
\hline 0.501 & 21.42 & 1 & 179 & $\mathrm{E}$ & 15 & 1 & 153 & $\mathrm{NE}$ & 44 & 31.08 & 143.8 & $\mathrm{SE}$ & 8.8 & 233 \\
\hline 0.501 & 22.93 & 1 & 139 & NE & 12 & 2 & 150.2 & $\mathrm{NE}$ & 41.4 & 29.7 & 153.8 & NW & 3.1 & 244 \\
\hline 0.501 & 23.43 & 2 & 150.2 & $\mathrm{NE}$ & 41.4 & 1 & 142 & $\mathrm{NE}$ & 24 & 17.92 & 158.5 & $\mathrm{~N}$ & 7.2 & 71 \\
\hline 0.501 & 23.93 & 1 & 142 & NE & 24 & 2 & 144.2 & $\mathrm{NE}$ & 23.4 & 1.06 & 86.8 & $\mathrm{E}$ & 20.1 & 357 \\
\hline 0.501 & 24.43 & 2 & 144.2 & $\mathrm{NE}$ & 23.4 & 2 & 153.7 & $\mathrm{NE}$ & 30.5 & 8.24 & 177.7 & $\mathrm{~N}$ & 13.5 & 270 \\
\hline 0.501 & 24.93 & 2 & 153.7 & $\mathrm{NE}$ & 30.5 & 1 & 156 & $\mathrm{NE}$ & 30 & 1.24 & 91.7 & $\mathrm{E}$ & 27.5 & 358 \\
\hline 0.501 & 38.97 & 1 & 160 & $\mathrm{E}$ & 25 & 1 & 157 & $\mathrm{NE}$ & 33 & 8.13 & 149.4 & SE & 4.9 & 238 \\
\hline 0.501 & 39.47 & 1 & 157 & NE & 33 & 3 & 148.6 & $\mathrm{NE}$ & 24.8 & 9.12 & 176.4 & $\mathrm{~N}$ & 12.2 & 90 \\
\hline 0.501 & 39.97 & 3 & 148.6 & NE & 24.8 & 2 & 159.6 & $\mathrm{E}$ & 24.4 & 4.6 & 70.2 & $\mathrm{E}$ & 24.4 & 339 \\
\hline 0.501 & 40.47 & 2 & 159.6 & $\mathrm{E}$ & 24.4 & 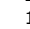 & 150 & $\mathrm{NE}$ & 23 & 4.09 & 43.8 & NE & 22.2 & 135 \\
\hline 0.501 & 40.97 & 1 & 150 & NE & 23 & 1 & 151 & $\mathrm{NE}$ & 19 & 4.02 & 145.7 & $\mathrm{SE}$ & 1.8 & 55 \\
\hline 0.501 & 41.47 & 1 & 151 & $\mathrm{NE}$ & 19 & 1 & 155 & $\mathrm{NE}$ & 26 & 7.16 & 164.5 & $\mathrm{~N}$ & 4.6 & 255 \\
\hline 0.501 & 41.97 & 1 & 155 & $\mathrm{NE}$ & 26 & 3 & 153.5 & $\mathrm{NE}$ & 22 & 4.05 & 162.2 & $\mathrm{~N}$ & 3.5 & 73 \\
\hline 0.501 & 42.47 & 3 & 153.5 & $\mathrm{NE}$ & 22 & 2 & 152.5 & $\mathrm{NE}$ & 24 & 2.04 & 142.9 & SE & 4.2 & 232 \\
\hline 0.501 & 42.98 & 2 & 152.5 & $\mathrm{NE}$ & 24 & 3 & 155.4 & $\mathrm{NE}$ & 22.2 & 2.12 & 123.4 & $\mathrm{SE}$ & 12.2 & 32 \\
\hline 0.501 & 43.48 & 3 & 155.4 & $\mathrm{NE}$ & 22.2 & 1 & 152 & $\mathrm{NE}$ & 22 & 1.3 & 53.7 & $\mathrm{NE}$ & 21.8 & 145 \\
\hline 0.501 & 43.98 & 1 & 152 & $\mathrm{NE}$ & 22 & 1 & 147 & $\mathrm{NE}$ & 23 & 2.16 & 89 & $\mathrm{E}$ & 19.8 & 177 \\
\hline 0.501 & 55.5 & 7 & 158.2 & $\mathrm{E}$ & 33.1 & $\varepsilon$ & 157.5 & $\mathrm{NE}$ & 28 & 5.15 & 161.3 & $\mathrm{~N}$ & 2 & 72 \\
\hline 0.501 & 56.01 & 8 & 157.5 & $\mathrm{NE}$ & 28 & 7 & 144.1 & $\mathrm{NE}$ & 31.2 & 7.33 & 90 & $\mathrm{E}$ & 26.1 & 177 \\
\hline 0.631 & 0.43 & 4 & 171 & $\mathrm{E}$ & 33.4 & 3 & 167 & $\mathrm{E}$ & 34.6 & 2.55 & 111.1 & $\mathrm{E}$ & 29.7 & 198 \\
\hline 0.631 & 1.06 & 3 & 167 & $\mathrm{E}$ & 34.6 & 2 & 153.3 & $\mathrm{NE}$ & 33.5 & 7.72 & 59.9 & NE & 33.4 & 153 \\
\hline 0.631 & 1.69 & 2 & 153.3 & $\mathrm{NE}$ & $=33.5$ & 2 & 156 & $\mathrm{NE}$ & 38.5 & 5.26 & 169.1 & $\mathrm{~N}$ & 10.2 & 262 \\
\hline 0.631 & 5.48 & 1 & 178 & E & 28 & 1 & 150 & $\mathrm{NE}$ & 30 & 13.61 & 83.4 & $E$ & 27.9 & 174 \\
\hline 0.631 & 7.37 & 1 & 149 & $\mathrm{NE}$ & 27 & 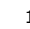 & 158 & $\mathrm{E}$ & 39 & 12.93 & 172.6 & $\mathrm{~N}$ & 11.5 & 265 \\
\hline 0.631 & 11.79 & 6 & 169.1 & E & 33.8 & 7 & 169.8 & $\mathrm{E}$ & 24.7 & 9.09 & 167.6 & $\mathrm{~s}$ & 1 & 77 \\
\hline 0.631 & 12.42 & 7 & 169.8 & E & 24.7 & 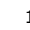 & 169 & E & 49 & 24.28 & 168.5 & $\mathrm{~S}$ & 0.6 & 258 \\
\hline 0.631 & 13.05 & 1 & 169 & $\mathrm{E}$ & 49 & 3 & 4.3 & $\mathrm{E}$ & 64.4 & 19.98 & 21.5 & $\mathrm{~N}$ & 31.7 & 303 \\
\hline 0.631 & 13.68 & 3 & 4.3 & E & 64.4 & 2 & 178.5 & E & 59 & 7.45 & 25.3 & NE & 36.9 & 136 \\
\hline 0.631 & 14.31 & 2 & 178.5 & $\mathrm{E}$ & 59 & 2 & 156.4 & $\mathrm{NE}$ & 21.2 & 39.92 & 4.9 & $\mathrm{~N}$ & 10.5 & 101 \\
\hline 0.631 & 21.25 & 1 & 145 & $\mathrm{NE}$ & 29 & 2 & 160 & E & 29 & 7.24 & 62.5 & NE & 28.8 & 332 \\
\hline 0.631 & 21.88 & 2 & 160 & E & 29 & 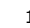 & 139 & $\mathrm{NE}$ & 12 & 18.26 & 172 & $\mathrm{~N}$ & 6.6 & 84 \\
\hline 0.631 & 22.51 & 1 & 139 & $\mathrm{NE}$ & 12 & 1 & 155 & $\mathrm{NE}$ & 44 & 32.6 & 159.4 & $\mathrm{~N}$ & 4.2 & 250 \\
\hline 0.631 & 23.14 & 1 & 155 & $\mathrm{NE}$ & $=44$ & 2 & 143.8 & $\mathrm{NE}$ & 31.5 & 14.22 & 173 & $\mathrm{~N}$ & 16.6 & 89 \\
\hline 0.631 & 23.77 & 2 & 143.8 & $\mathrm{NE}$ & 31.5 & 2 & 144.2 & $\mathrm{NE}$ & 23.4 & 8.04 & 142.8 & SE & 0.6 & 53 \\
\hline 0.631 & 24.4 & 2 & 144.2 & $\mathrm{NE}$ & 23.4 & 2 & 153.7 & $\mathrm{NE}$ & 30.5 & 8.24 & 177.7 & $\mathrm{~N}$ & 13.5 & 270 \\
\hline 0.631 & 25.04 & 2 & 153.7 & $\mathrm{NE}$ & 30.5 & 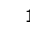 & 156 & $\mathrm{NE}$ & 30 & 1.24 & 91.7 & $\mathrm{E}$ & 27.5 & 358 \\
\hline 0.631 & 38.92 & 1 & 160 & $\mathrm{E}$ & 25 & 2 & 152.7 & $\mathrm{NE}$ & 31.4 & 7.28 & 130.9 & SE & 12.8 & 218 \\
\hline 0.631 & 39.55 & 2 & 152.7 & $\mathrm{NE}$ & 31.4 & 3 & 155.2 & $\mathrm{NE}$ & 22.9 & 8.57 & 147.2 & $\mathrm{SE}$ & 3.4 & 56 \\
\hline 0.631 & 40.18 & 3 & 155.2 & $\mathrm{NE}$ & 22.9 & 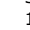 & 153 & $\mathrm{NE}$ & 24 & 1.37 & 117.6 & $\mathrm{SE}$ & 14.4 & 206 \\
\hline 0.631 & 40.81 & 1 & 153 & $\mathrm{NE}$ & 24 & 2 & 150.5 & $\mathrm{NE}$ & 21 & 3.15 & 168.4 & $\mathrm{~N}$ & 6.8 & 80 \\
\hline 0.631 & 41.44 & 2 & 150.5 & $\mathrm{NE}$ & 21 & 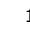 & 155 & $\mathrm{NE}$ & 26 & 5.32 & 171.1 & $\mathrm{~N}$ & 7.7 & 262 \\
\hline 0.631 & 42.07 & 1 & 155 & $\mathrm{NE}$ & 26 & 3 & 153.5 & $\mathrm{NE}$ & 22 & 4.05 & 162.2 & $\mathrm{~N}$ & 3.5 & 73 \\
\hline 0.631 & 42.7 & 3 & 153.5 & $\mathrm{NE}$ & $=22$ & 4 & 151.7 & $\mathrm{NE}$ & 22.2 & 0.73 & 83.5 & $\mathrm{E}$ & 20.8 & 169 \\
\hline 0.631 & 43.33 & 4 & 151.7 & $\mathrm{NE}$ & 22.2 & 2 & 157.9 & $\mathrm{E}$ & 23.9 & 2.97 & 28.5 & NE & 18.9 & 300 \\
\hline 0.631 & 43.96 & 2 & 157.9 & $\mathrm{E}$ & 23.9 & 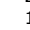 & 147 & $\mathrm{NE}$ & 23 & 4.44 & 49.8 & $\mathrm{NE}$ & 22.8 & 141 \\
\hline 0.631 & 55.32 & 4 & 160.5 & $\mathrm{E}$ & 34.4 & 1 & 155.8 & $\mathrm{NE}$ & 28.9 & 6.03 & 179.1 & $\mathrm{~N}$ & 12.3 & 93 \\
\hline 0.631 & 55.95 & 10 & 155.8 & $\mathrm{NE}$ & 28.9 & $\varepsilon$ & 146.8 & $\mathrm{NE}$ & 30.9 & 4.88 & 88.3 & $\mathrm{E}$ & 27 & 176 \\
\hline 0.794 & 0.79 & 7 & 169.2 & E & 33.9 & 2 & 153.3 & $\mathrm{NE}$ & 33.5 & 8.83 & 67.7 & $E$ & 33.4 & 160 \\
\hline 0.794 & 1.58 & 2 & 153.3 & $\mathrm{NE}$ & 33.5 & 2 & 156 & $\mathrm{NE}$ & 38.5 & 5.26 & 169.1 & $\mathrm{~N}$ & 10.2 & 262 \\
\hline 0.794 & 5.55 & 1 & 178 & $\mathrm{E}$ & 28 & 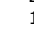 & 150 & $\mathrm{NE}$ & 30 & 13.61 & 83.4 & $\mathrm{E}$ & 27.9 & 174 \\
\hline
\end{tabular}




\begin{tabular}{|c|c|c|c|c|c|c|c|c|c|c|c|c|c|c|}
\hline 1 & 11.69 & 5 & 166.7 & $\mathrm{E}$ & 30.2 & 8 & 171.5 & $\mathrm{E}$ & 28.5 & 2.92 & 119.7 & $\mathrm{SE}$ & 23.1 & 25 \\
\hline 1 & 12.69 & 8 & 171.5 & E & 28.5 & 4 & 1.2 & $E$ & 59.9 & 32.07 & 5.7 & $\mathrm{~N}$ & 7.6 & 277 \\
\hline 1 & 13.69 & 4 & 1.2 & $\mathrm{E}$ & 59.9 & 3 & 174.7 & $\mathrm{E}$ & 45.4 & 15.38 & 10.3 & $\mathrm{~N}$ & 15.2 & 109 \\
\hline 1 & 14.69 & 3 & 174.7 & $\mathrm{E}$ & 45.4 & 1 & 164 & $E$ & 29 & 17.57 & 7.2 & $\mathrm{~N}$ & 12.3 & 102 \\
\hline 1 & 15.69 & 1 & 164 & $\mathrm{E}$ & 29 & 1 & 154 & NE & 50 & 21.88 & 145.5 & $\mathrm{SE}$ & 10 & 233 \\
\hline 1 & 19.69 & 1 & 151 & $\mathrm{NE}$ & 26 & 1 & 131 & $\mathrm{NE}$ & 47 & 23.9 & 115.8 & $\mathrm{SE}$ & 15.7 & 203 \\
\hline 1 & 20.69 & 1 & 131 & $\mathrm{NE}$ & 47 & 2 & 156.7 & $\mathrm{NE}$ & 21.2 & 29.05 & 117.9 & $\mathrm{SE}$ & 13.7 & 22 \\
\hline 1 & 21.69 & 2 & 156.7 & $\mathrm{NE}$ & 21.2 & 2 & 149.8 & $\mathrm{NE}$ & 27.9 & 7.25 & 131.8 & SE & 9.3 & 220 \\
\hline 1 & 22.69 & 2 & 149.8 & $\mathrm{NE}$ & 27.9 & 3 & 148.3 & $\mathrm{NE}$ & 35.6 & 7.76 & 144.1 & $\mathrm{SE}$ & 3 & 233 \\
\hline 1 & 23.69 & 3 & 148.3 & $\mathrm{NE}$ & 35.6 & 3 & 147.7 & $\mathrm{NE}$ & 22.9 & 12.72 & 149.2 & NW & 0.6 & 59 \\
\hline 1 & 24.69 & 3 & 147.7 & $\mathrm{NE}$ & 22.9 & 2 & 154.3 & $\mathrm{NE}$ & 34.5 & 12.02 & 164.7 & $\mathrm{~N}$ & 7 & 256 \\
\hline 1 & 29.69 & 1 & 151 & $\mathrm{NE}$ & 44 & 1 & 152 & $\mathrm{NE}$ & 36 & 8.03 & 148 & $\mathrm{SE}$ & 2.9 & 57 \\
\hline 1 & 36.69 & 1 & 152 & $\mathrm{NE}$ & 22 & 1 & 158 & $E$ & 23 & 2.5 & 39.8 & $\mathrm{NE}$ & 20.5 & 311 \\
\hline 1 & 39.69 & 4 & 150.3 & $\mathrm{NE}$ & 27.4 & 3 & 161.4 & $\mathrm{E}$ & 23.9 & 5.93 & 104.8 & $E$ & 20.3 & 11 \\
\hline 1 & 40.69 & 3 & 161.4 & $\mathrm{E}$ & 23.9 & 2 & 150.5 & $\mathrm{NE}$ & 21 & 5.05 & 29.1 & $\mathrm{NE}$ & 18.2 & 122 \\
\hline 1 & 41.69 & 2 & 150.5 & $\mathrm{NE}$ & 21 & 4 & 153.9 & $\mathrm{NE}$ & 23 & 2.38 & 3.2 & $\mathrm{~N}$ & 11.7 & 275 \\
\hline 1 & 42.69 & 4 & 153.9 & $\mathrm{NE}$ & 23 & 6 & 153.8 & $\mathrm{NE}$ & 22.8 & 0.24 & 160.5 & $\mathrm{~N}$ & 2.8 & 75 \\
\hline 1 & 43.69 & 6 & 153.8 & $\mathrm{NE}$ & 22.8 & 1 & 147 & $\mathrm{NE}$ & 23 & 2.67 & 65.9 & $\mathrm{NE}$ & 22.7 & 155 \\
\hline 1 & 55.69 & 10 & 156.6 & $\mathrm{NE}$ & 32.6 & 12 & 150.7 & $\mathrm{NE}$ & 28.6 & 4.95 & 7.2 & $\mathrm{~N}$ & 18 & 101 \\
\hline 1.259 & 1.12 & 8 & 167.3 & $\mathrm{E}$ & 33.1 & 3 & 155.7 & $\mathrm{NE}$ & 38.7 & 8.8 & 116.8 & SE & 26.7 & 202 \\
\hline 1.259 & 6.16 & 2 & 163.6 & $\mathrm{E}$ & 28.3 & 1 & 149 & $\mathrm{NE}$ & 27 & 6.85 & 54.3 & $\mathrm{NE}$ & 26.9 & 146 \\
\hline 1.259 & 7.42 & 1 & 149 & $\mathrm{NE}$ & 27 & 1 & 158 & $E$ & 39 & 12.93 & 172.6 & $\mathrm{~N}$ & 11.5 & 265 \\
\hline 1.259 & 12.45 & 13 & 169.5 & $\mathrm{E}$ & 29.2 & 4 & 1.2 & $\mathrm{E}$ & 59.9 & 31.68 & 6.8 & $\mathrm{~N}$ & 9.4 & 279 \\
\hline 1.259 & 13.71 & 4 & 1.2 & $\mathrm{E}$ & 59.9 & 4 & 172.2 & $\mathrm{E}$ & 40.1 & 20.9 & 9.7 & $\mathrm{~N}$ & 14.2 & 108 \\
\hline 1.259 & 14.97 & 4 & 172.2 & $\mathrm{E}$ & 40.1 & 1 & 154 & $\mathrm{NE}$ & 50 & 16.16 & 120 & $\mathrm{SE}$ & 33.7 & 203 \\
\hline 1.259 & 20.01 & 2 & 138.5 & $\mathrm{NE}$ & 36.1 & 1 & 145 & $\mathrm{NE}$ & 29 & 7.92 & 119 & $\mathrm{SE}$ & 13.6 & 25 \\
\hline 1.259 & 21.27 & 1 & 145 & $\mathrm{NE}$ & 29 & 3 & 156.3 & $\mathrm{NE}$ & 22.6 & 8.03 & 115.6 & SE & 15.2 & 22 \\
\hline 1.259 & 22.52 & 3 & 156.3 & $\mathrm{NE}$ & 22.6 & 3 & 148.3 & $\mathrm{NE}$ & 35.6 & 13.51 & 137.4 & $\mathrm{SE}$ & 7.7 & 226 \\
\hline 1.259 & 23.78 & 3 & 148.3 & $\mathrm{NE}$ & 35.6 & 4 & 149.5 & $\mathrm{NE}$ & 26.8 & 8.8 & 145.4 & SE & 2.1 & 55 \\
\hline 1.259 & 25.04 & 4 & 149.5 & $\mathrm{NE}$ & 26.8 & 1 & 156 & $\mathrm{NE}$ & 30 & 4.42 & 13.2 & $\mathrm{~N}$ & 19.3 & 286 \\
\hline 1.259 & 30.08 & 1 & 151 & $\mathrm{NE}$ & 44 & 1 & 152 & $\mathrm{NE}$ & 36 & 8.03 & 148 & $\mathrm{SE}$ & 2.9 & 57 \\
\hline 1.259 & 35.11 & 1 & 164 & $\mathrm{E}$ & 22 & 1 & 152 & $\mathrm{NE}$ & 22 & 4.49 & 68 & $\mathrm{E}$ & 21.9 & 158 \\
\hline 1.259 & 36.37 & 1 & 152 & $\mathrm{NE}$ & 22 & 1 & 158 & $\mathrm{E}$ & 23 & 2.5 & 39.8 & $\mathrm{NE}$ & 20.5 & 311 \\
\hline 1.259 & 37.63 & 1 & 158 & $\mathrm{E}$ & 23 & 1 & 160 & $E$ & 25 & 2.16 & 179.4 & $N$ & 8.8 & 271 \\
\hline 1.259 & 38.89 & 1 & 160 & $\mathrm{E}$ & 25 & 5 & 154 & $\mathrm{NE}$ & 26.3 & 2.92 & 96.6 & $\mathrm{E}$ & 22.6 & 184 \\
\hline 1.259 & 40.15 & 5 & 154 & $\mathrm{NE}$ & 26.3 & 3 & 151.4 & $\mathrm{NE}$ & 22 & 4.47 & 165.4 & $\mathrm{~N}$ & 5.6 & 77 \\
\hline 1.259 & 41.41 & 3 & 151.4 & $\mathrm{NE}$ & 22 & 4 & 153.9 & $\mathrm{NE}$ & 23 & 1.39 & 14.5 & $\mathrm{~N}$ & 15.4 & 286 \\
\hline 1.259 & 42.67 & 4 & 153.9 & $\mathrm{NE}$ & 23 & 6 & 153.8 & $\mathrm{NE}$ & 22.8 & 0.24 & 160.5 & $\mathrm{~N}$ & 2.8 & 75 \\
\hline 1.259 & 43.93 & 6 & 153.8 & $\mathrm{NE}$ & 22.8 & 1 & 147 & $\mathrm{NE}$ & 23 & 2.67 & 65.9 & $\mathrm{NE}$ & 22.7 & 155 \\
\hline 1.259 & 55.26 & 2 & 168.4 & $\mathrm{E}$ & 34 & 20 & 152 & $\mathrm{NE}$ & 30.2 & 9.48 & 43.4 & $\mathrm{NE}$ & 28.9 & 138 \\
\hline 1.585 & 1.25 & 8 & 167.3 & $\mathrm{E}$ & 33.1 & 3 & 155.7 & $\mathrm{NE}$ & 38.7 & 8.8 & 116.8 & SE & 26.7 & 202 \\
\hline 1.585 & 6 & 2 & 163.6 & $\mathrm{E}$ & 28.3 & 1 & 149 & $\mathrm{NE}$ & 27 & 6.85 & 54.3 & $\mathrm{NE}$ & 26.9 & 146 \\
\hline 1.585 & 7.59 & 1 & 149 & $\mathrm{NE}$ & 27 & 1 & 158 & $\mathrm{E}$ & 39 & 12.93 & 172.6 & $N$ & 11.5 & 265 \\
\hline 1.585 & 12.34 & 12 & 168.6 & $\mathrm{E}$ & 27.6 & 5 & 180 & $E$ & 57.2 & 30.47 & 5.7 & $\mathrm{~N}$ & 8.8 & 278 \\
\hline 1.585 & 13.93 & 5 & 180 & $\mathrm{E}$ & 57.2 & 4 & 172.2 & $E$ & 40.1 & 18.01 & 9.1 & $\mathrm{~N}$ & 13.8 & 106 \\
\hline 1.585 & 15.51 & 4 & 172.2 & $\mathrm{E}$ & 40.1 & 1 & 154 & $\mathrm{NE}$ & 50 & 16.16 & 120 & SE & 33.7 & 203 \\
\hline 1.585 & 20.27 & 2 & 138.5 & $\mathrm{NE}$ & 36.1 & 3 & 154.7 & $\mathrm{NE}$ & 28.8 & 11.31 & 100.9 & $E$ & 24 & 5 \\
\hline 1.585 & 21.85 & 3 & 154.7 & $\mathrm{NE}$ & 28.8 & 3 & 148.7 & $\mathrm{NE}$ & 31.9 & 4.31 & 111.3 & $\mathrm{E}$ & 20.7 & 198 \\
\hline 1.585 & 23.44 & 3 & 148.7 & $\mathrm{NE}$ & 31.9 & 5 & 148.1 & $\mathrm{NE}$ & 26.2 & 5.71 & 151.1 & NW & 1.5 & 61 \\
\hline 1.585 & 25.02 & 5 & 148.1 & $\mathrm{NE}$ & 26.2 & 1 & 156 & $\mathrm{NE}$ & 30 & 5.3 & 13 & $\mathrm{~N}$ & 19.1 & 286 \\
\hline 1.585 & 29.77 & 1 & 151 & $\mathrm{NE}$ & 44 & 1 & 152 & $\mathrm{NE}$ & 36 & 8.03 & 148 & SE & 2.9 & 57 \\
\hline 1.585 & 34.53 & 1 & 164 & $\mathrm{E}$ & 22 & 1 & 152 & $\mathrm{NE}$ & 22 & 4.49 & 68 & $E$ & 21.9 & 158 \\
\hline 1.585 & 36.11 & 1 & 152 & $\mathrm{NE}$ & 22 & 1 & 158 & $\mathrm{E}$ & 23 & 2.5 & 39.8 & $\mathrm{NE}$ & 20.5 & 311 \\
\hline 1.585 & 37.7 & 1 & 158 & $\mathrm{E}$ & 23 & 2 & 158.3 & $E$ & 29 & 5.99 & 159.3 & $\mathrm{~N}$ & 0.6 & 249 \\
\hline 1.585 & 39.28 & 2 & 158.3 & $\mathrm{E}$ & 29 & 5 & 153 & $\mathrm{NE}$ & 24.5 & 5.06 & 1.3 & $\mathrm{~N}$ & 12.2 & 94 \\
\hline 1.585 & 40.87 & 5 & 153 & $\mathrm{NE}$ & 24.5 & 6 & 152.8 & $\mathrm{NE}$ & 22.3 & 2.21 & 154.5 & NW & 0.7 & 65 \\
\hline 1.585 & 42.45 & 6 & 152.8 & $\mathrm{NE}$ & 22.3 & 7 & 152.9 & $\mathrm{NE}$ & 22.8 & 0.46 & 154.1 & NW & 0.5 & 247 \\
\hline 1.585 & 55.13 & 1 & 170 & $\mathrm{E}$ & 32 & 21 & 152.8 & $\mathrm{NE}$ & 30.5 & 9 & 60.2 & $\mathrm{NE}$ & 30.5 & 153 \\
\hline 1.995 & 1.25 & 8 & 167.3 & $\mathrm{E}$ & 33.1 & 3 & 155.7 & $\mathrm{NE}$ & 38.7 & 8.8 & 116.8 & SE & 26.7 & 202 \\
\hline 1.995 & 7.24 & 3 & 158.8 & $\mathrm{E}$ & 27.7 & 1 & 158 & $\mathrm{E}$ & 39 & 11.33 & 156.6 & SE & 1.1 & 246 \\
\hline 1.995 & 13.23 & 15 & 170.1 & $\mathrm{E}$ & 31.9 & 6 & 178.4 & $E$ & 52.7 & 21.52 & 5.8 & $\mathrm{~N}$ & 9.6 & 278 \\
\hline 1.995 & 15.22 & 6 & 178.4 & $\mathrm{E}$ & 52.7 & 1 & 154 & $\mathrm{NE}$ & 50 & 19.22 & 63.5 & $\mathrm{NE}$ & 50 & 163 \\
\hline 1.995 & 21.21 & 3 & 140.5 & $\mathrm{NE}$ & 33.6 & 4 & 155.8 & $\mathrm{NE}$ & 28.4 & 9.37 & 95.5 & $\mathrm{E}$ & 25.1 & 0 \\
\hline 1.995 & 23.2 & 4 & 155.8 & $\mathrm{NE}$ & 28.4 & 7 & 148.7 & $\mathrm{NE}$ & 28.6 & 3.35 & 65.5 & NE & 28.4 & 156 \\
\hline 1.995 & 29.19 & 1 & 151 & $\mathrm{NE}$ & 44 & 1 & 152 & $\mathrm{NE}$ & 36 & 8.03 & 148 & SE & 2.9 & 57 \\
\hline 1.995 & 35.17 & 1 & 164 & $\mathrm{E}$ & 22 & 1 & 152 & $\mathrm{NE}$ & 22 & 4.49 & 68 & $\mathrm{E}$ & 21.9 & 158 \\
\hline 1.995 & 37.17 & 1 & 152 & $\mathrm{NE}$ & 22 & 2 & 159 & $E$ & 24 & 3.4 & 27.3 & $\mathrm{NE}$ & 18.4 & 299 \\
\hline 1.995 & 39.16 & 2 & 159 & $\mathrm{E}$ & 24 & 7 & 153.3 & $\mathrm{NE}$ & 25.5 & 2.83 & 101 & $\mathrm{E}$ & 20.7 & 189 \\
\hline 1.995 & 41.16 & 7 & 153.3 & $\mathrm{NE}$ & 25.5 & 8 & 153.1 & $\mathrm{NE}$ & 22.6 & 2.89 & 154.8 & NW & 0.7 & 65 \\
\hline 1.995 & 43.15 & 8 & 153.1 & $\mathrm{NE}$ & 22.6 & 4 & 153 & $\mathrm{NE}$ & 22.4 & 0.26 & 162.2 & $\mathrm{~N}$ & 3.7 & 74 \\
\hline 2.512 & 1.82 & 9 & 165.7 & $\mathrm{E}$ & 33.7 & 2 & 156 & $\mathrm{NE}$ & 38.5 & 7.49 & 117 & SE & 26.6 & 202 \\
\hline 2.512 & 4.33 & 2 & 156 & $\mathrm{NE}$ & 38.5 & 3 & 158.8 & $\mathrm{E}$ & 27.7 & 10.92 & 150.7 & SE & 4.2 & 59 \\
\hline 2.512 & 6.84 & 3 & 158.8 & $\mathrm{E}$ & 27.7 & 1 & 158 & $\mathrm{E}$ & 39 & 11.33 & 156.6 & $\mathrm{SE}$ & 1.1 & 246 \\
\hline 2.512 & 9.35 & 1 & 158 & $\mathrm{E}$ & 39 & 7 & 171.5 & $E$ & 32.9 & 9.98 & 117.9 & SE & 27.5 & 21 \\
\hline 2.512 & 11.86 & 7 & 171.5 & $\mathrm{E}$ & 32.9 & 12 & 175.2 & $\mathrm{E}$ & 43.4 & 10.72 & 3.2 & $\mathrm{~N}$ & 7.4 & 275 \\
\hline 2.512 & 14.37 & 12 & 175.2 & $\mathrm{E}$ & 43.4 & 3 & 155.3 & $\mathrm{NE}$ & 30.6 & 17.4 & 22.7 & NE & 23.6 & 121 \\
\hline 2.512 & 16.89 & 3 & 155.3 & $\mathrm{NE}$ & 30.6 & 1 & 151 & $\mathrm{NE}$ & 26 & 5.06 & 174.3 & $\mathrm{~N}$ & 10.9 & 87 \\
\hline 2.512 & 19.4 & 1 & 151 & $\mathrm{NE}$ & 26 & 4 & 146.7 & $\mathrm{NE}$ & 33.1 & 7.38 & 134.2 & SE & 8 & 222 \\
\hline 2.512 & 21.91 & 4 & 146.7 & $\mathrm{NE}$ & 33.1 & 6 & 146.3 & $\mathrm{NE}$ & 27.5 & 5.6 & 148.1 & NW & 0.9 & 59 \\
\hline
\end{tabular}




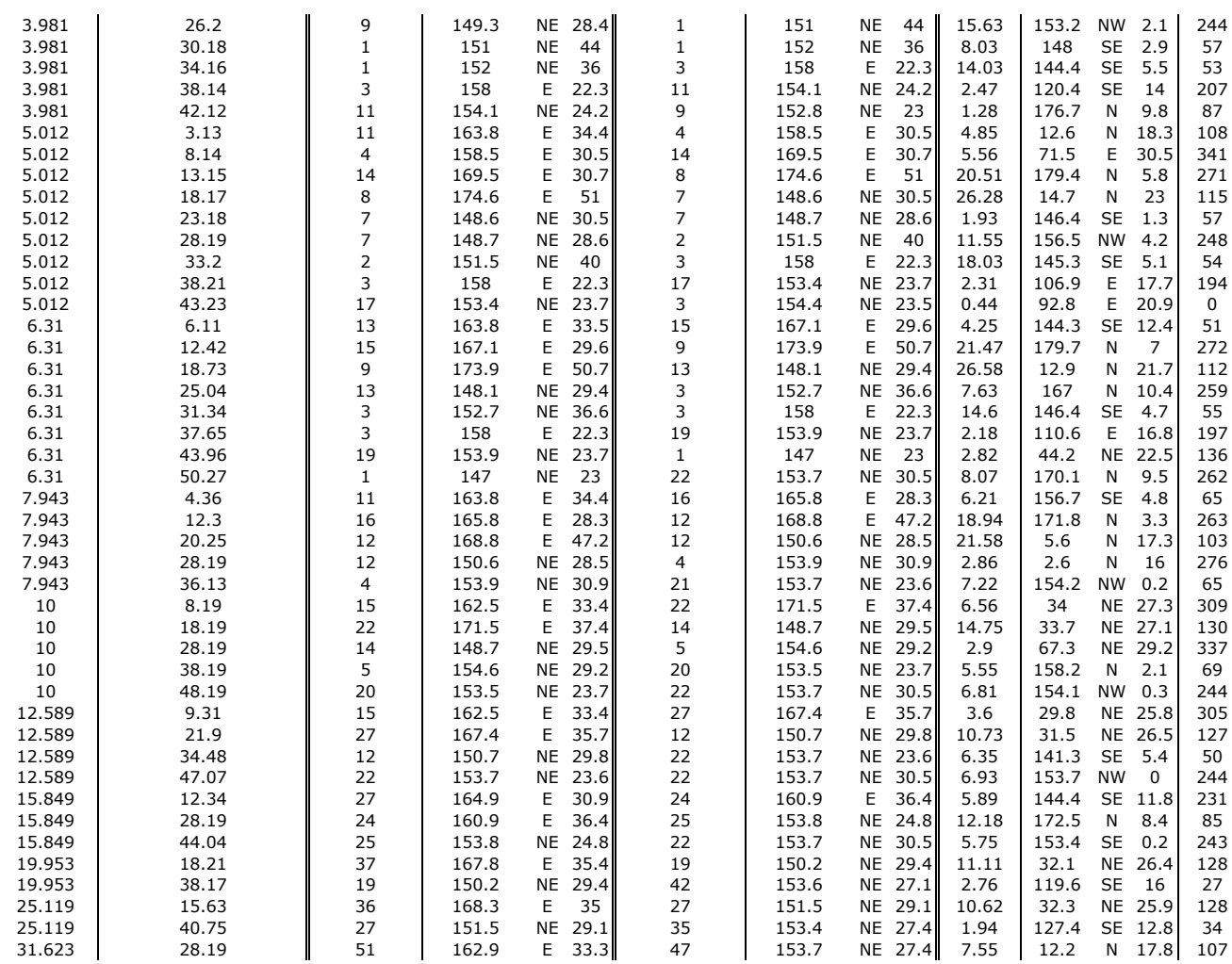


Section I

Tilt number window size elevation tilt angle tilt axis tilt way

\begin{tabular}{c|c|c|c|c}
$(\mathrm{m})$ & $(\mathrm{m})$ & $\left(^{\circ}\right)$ & $\left(^{\circ}\right)$ & $\left(^{\circ}\right)$ \\
39.811 & 36.91 & 5.1 & 47.3 & 140 \\
3
\end{tabular}

\begin{tabular}{l|l|l|l|l}
39.811 & 36.91 & 5.1 & 47.3 & 140 \\
31.623 & 21.09 & 7.2 & 29.4 & 123
\end{tabular}

\begin{tabular}{l|l|l|l|l}
25.119 & 24.35 & 6.2 & 53.6 & 146
\end{tabular}

\begin{tabular}{l|l|l|l|l}
31.623 & 52.72 & 7.8 & 148.2 & 58 \\
\hline
\end{tabular}

\begin{tabular}{l|l|l|l|l}
25.119 & 49.46 & 3 & 155.5 & 66 \\
\hline
\end{tabular}

\begin{tabular}{c|c|c|c|c}
19.953 & 56.86 & 9.5 & 137.3 & 46 \\
15.849 & 60.68 & 15.6 & 140.4 & 49
\end{tabular}

\begin{tabular}{l|l|l|l|l}
12.589 & 62.08 & 13.7 & 139.2 & 48 \\
\hline & 56.9 & 10.3 & 135.4 & 44
\end{tabular}

\begin{tabular}{c|c|c|c|c|}
10 & 56.9 & 10.3 & 135.4 & 44 \\
10 & 66.9 & 7.7 & 141.9 & 51
\end{tabular}

\begin{tabular}{c|c|c|c|c}
10 & 66.9 & 7.7 & 141.9 & 51 \\
7.943 & 52.79 & 9.7 & 139.8 & 48 \\
\hline
\end{tabular}

\begin{tabular}{l|l|l|l|l|l}
6.31 & 55.83 & 9.8 & 137.5 & 46 \\
\hline
\end{tabular}

\begin{tabular}{l|l|l|l|l}
5.012 & 54.45 & 9.8 & 137.5 & 46 \\
\hline
\end{tabular}

\begin{tabular}{l|l|l|l|r}
3.981 & 54.82 & 10 & 150.8 & 61 \\
19.953 & 16.95 & 6.8 & 16.5 & 110 \\
\hline
\end{tabular}

\begin{tabular}{l|l|l|l|l|}
15.849 & 13.13 & 5.1 & 3.3 & 95 \\
\hline
\end{tabular}

\begin{tabular}{l|l|l|l|l|l}
12.589 & 11.73 & 4.6 & 172.8 & 84 \\
\hline
\end{tabular}

\begin{tabular}{c|c|c|c|c}
10 & 16.91 & 7.3 & 179.6 & 92 \\
7.943 & 21.02 & 6.5 & 174.5 & 86 \\
\hline
\end{tabular}

\begin{tabular}{l|l|l|l|l}
6.31 & 17.98 & 9.5 & 174.5 & 86 \\
6.1 & 174.7 & 86
\end{tabular}

\begin{tabular}{l|l|l|l|l}
5.012 & 14.35 & 6.6 & 164.3 & 75 \\
\hline
\end{tabular}

\begin{tabular}{l|l|l|l|l}
5.012 & 19.36 & 6.6 & 165.9 & 76 \\
\hline 3.981 & 15.01 & 7.9 & 148.7 & 77 \\
\hline
\end{tabular}

\begin{tabular}{l|l|l|l|l}
5.012 & 19.36 & 6.6 & 165.9 & 76 \\
3.981 & 15.01 & 7.9 & 166.7 & 77 \\
3.162 & 18.99 & 3.9 & 148.6 & 57
\end{tabular}

\begin{tabular}{l|l|l|l|l}
3.162 & 14.77 & 13.3 & 161.1 & 70 \\
\hline
\end{tabular}

\begin{tabular}{|l|l|l|l|l}
2.512 & 14.3 & 12.4 & 155.3 & 63 \\
\hline
\end{tabular}

\begin{tabular}{l|l|l|l|l}
15.849 & 44.83 & 9.4 & 145.1 & 235 \\
12.589 & 49.49 & 3.5 & 143.6 & 233
\end{tabular}

\begin{tabular}{l|l|l|l|l|}
10 & 46.9 & 14.5 & 137.4 & 227 \\
\hline
\end{tabular}

\begin{tabular}{l|l|l|l|l}
7.943 & 44.85 & 14.3 & 145.3 & 235 \\
\hline
\end{tabular}

\begin{tabular}{l|l|l|l|l|l}
7.31 & 43.21 & 14.4 & 149.4 & 239 \\
\hline
\end{tabular}

\begin{tabular}{l|l|l|l|l|l}
5.012 & 44.42 & 14.7 & 141.6 & 231 \\
\hline
\end{tabular}

\begin{tabular}{c|c|c|c|c}
12.589 & 24.32 & 4.2 & 83.3 & 173 \\
10 & 26.91 & 5.5 & 93 & 182
\end{tabular}

\begin{tabular}{c|c|c|c|c}
10 & 26.91 & 5.5 & 93 & 182 \\
7.943 & 28.96 & 5.6 & 91.1 & 179
\end{tabular}

\begin{tabular}{c|c|c|c|c}
7.31 & 30.6 & 4.5 & 69.3 & 159 \\
5.012 & 29.39 & 1.8 & 79.5 & 167
\end{tabular}

\begin{tabular}{l|c|c|c|c}
5.012 & 29.39 & 1.8 & 79.5 & 167 \\
10 & 6.91 & 1.5 & 157.9 & 247
\end{tabular}

\begin{tabular}{l|l|l|l|l}
10 & 6.91 & 1.5 & 157.9 & 247 \\
7.943 & 5.13 & 2.9 & 152.4 & 240
\end{tabular}

\begin{tabular}{c|c|c|c|c}
6.31 & 5.36 & 2.5 & 150.2 & 238 \\
5.012 & 9.34 & 3.4 & 136 & 222
\end{tabular}

\begin{tabular}{l|c|c|c|c}
5.012 & 9.34 & 3.4 & 136 & 222 \\
3.981 & 11.03 & 4.6 & 159.3 & 249 \\
\hline & 8.44 & 2.9 & 3.8 & 276
\end{tabular}

\begin{tabular}{|c|c|c|c|c}
3.162 & 8.44 & 2.9 & 3.8 & 276 \\
10 & 36.91 & 8.1 & 139.1 & 48
\end{tabular}

\begin{tabular}{l|l|l|l|l}
7.943 & 36.91 & 8.1 & 146.4 & 56
\end{tabular}

\begin{tabular}{c|c|c|c|c}
6.31 & 36.91 & 9 & 150.1 & 60 \\
\hline
\end{tabular}

\begin{tabular}{l|l|l|l|l}
5.012 & 39.41 & 7.2 & 128.1 & 37 \\
\hline
\end{tabular}

\begin{tabular}{l|l|l|l|l}
3.981 & 38.9 & 11.5 & 145.4 & 56 \\
\hline
\end{tabular}

\begin{tabular}{l|l|l|l|l}
7.943 & 68.68 & 7.4 & 142.7 & 52 \\
\hline 5.31 & 68.45 & 7.4 & 142.7 & 52 \\
\hline
\end{tabular}

\begin{tabular}{l|l|l|l|l}
\hline 6.31 & 68.45 & 7.4 & 142.7 & 52 \\
\hline
\end{tabular}

\begin{tabular}{l|l|l|l|l}
5.012 & 69.48 & 7.4 & 142.7 & 52 \\
3.981 & 66.76 & 7.6 & 156.2 & 66
\end{tabular}

\begin{tabular}{l|l|l|l|l}
3.981 & 66.76 & 7.6 & 156.2 & 66 \\
3.162 & 68.53 & 6.4 & 153.6 & 64 \\
\hline
\end{tabular}

\begin{tabular}{l|l|l|l|l}
6.31 & 24.29 & 3.8 & 139 & 227 \\
\hline
\end{tabular}

\begin{tabular}{l|l|l|l|l}
5.012 & 24.38 & 6.5 & 152.9 & 242 \\
\hline & 26.95 & 7.2 & 137.7 & 226
\end{tabular}

\begin{tabular}{l|l|l|l|l|}
3.981 & 26.95 & 7.2 & 137.7 & 226 \\
3.162 & 24.26 & 4.7 & 137.3 & 226 \\
\hline
\end{tabular}

\begin{tabular}{l|l|l|l|l}
2.512 & 24.35 & 4.3 & 137.3 & 226 \\
\hline .512 & 26.86 & 5.5 & 133.5 & 218
\end{tabular}

\begin{tabular}{l|c|c|c|c}
2.512 & 26.86 & 5.5 & 133.5 & 221 \\
\hline & 4.33 & 3.8 & 5.2 & 278
\end{tabular}

\begin{tabular}{c|c|c|c|c}
5.012 & 4.33 & 3.8 & 5.2 & 278 \\
3.981 & 3.07 & 7.1 & 24.3 & 297
\end{tabular}

\begin{tabular}{l|l|l|l|l}
3.981 & 3.07 & 7.1 & 24.3 & 297 \\
3.162 & 2.12 & 7.7 & 37.8 & 310
\end{tabular}

\begin{tabular}{l|l|l|l|l}
3.162 & 2.12 & 7.7 & 37.8 & 310 \\
\hline .512 & 1.74 & 9.3 & 42.7 & 315 \\
\hline
\end{tabular}

\begin{tabular}{l|l|l|l|l}
\hline .995 & 1.99 & 9.3 & 42.7 & 315 \\
\hline
\end{tabular}

\begin{tabular}{c|c|c|c|c}
3.981 & 42.88 & 16.4 & 9.2 & 280 \\
3.162 & 43.23 & 8.1 & 9.4 & 281 \\
\hline & &
\end{tabular}

\begin{tabular}{c|c|c|c|c|}
\hline .512 & 41.93 & 14.8 & 0.2 & 271 \\
\hline
\end{tabular}

\begin{tabular}{l|l|l|l|l}
1.995 & 41.89 & 11.7 & 175.4 & 266 \\
\hline
\end{tabular}

\begin{tabular}{c|c|c|c|c}
3.981 & 46.86 & 12.9 & 93.8 & 183 \\
\hline
\end{tabular}

\begin{tabular}{|l|l|l|l|l}
3.162 & 46.39 & 14 & 100.2 & 189 \\
\hline
\end{tabular}

\begin{tabular}{l|l|l|l|l|l}
2.512 & 46.95 & 23.7 & 79 & 172 \\
\hline
\end{tabular}

\begin{tabular}{l|l|l|l|l}
1.995 & 45.88 & 15.4 & 103.5 & 191 \\
\hline
\end{tabular}

\begin{tabular}{l|c|c|c|c}
1.585 & 47.21 & 23.7 & 79 & 172 \\
3.162 & 11.61 & 5.5 & 160.5 & 249 \\
\hline
\end{tabular}

\begin{tabular}{l|l|l|l|l}
3.162 & 11.61 & 5.5 & 160.5 & 249 \\
2.512 & 11.79 & 9 & 175.9 & 268
\end{tabular} 


\begin{tabular}{|c|c|c|c|c|c|}
\hline 53 & 0.794 & 50.01 & 2.6 & 153 & 245 \\
\hline 53 & 0.631 & 49.84 & 9.6 & 152.3 & 243 \\
\hline 53 & 0.501 & 49.94 & 9.6 & 152.3 & 243 \\
\hline 53 & 0.398 & 50.04 & 6.7 & 167.4 & 262 \\
\hline 63 & 1.585 & 40.87 & 14.4 & 55 & 324 \\
\hline 63 & 1.259 & 40.05 & 12.2 & 44.2 & 313 \\
\hline 63 & 1 & 39.9 & 6.5 & 24.1 & 294 \\
\hline 63 & 0.794 & 40.48 & 12.2 & 44.2 & 313 \\
\hline 63 & 0.631 & 41.01 & 16.5 & 64.2 & 333 \\
\hline 63 & 0.501 & 40.91 & 16.5 & 64.2 & 333 \\
\hline 63 & 0.398 & 40.89 & 12 & 62.9 & 332 \\
\hline 63 & 0.316 & 41.02 & 16.5 & 64.2 & 333 \\
\hline 63 & 0.251 & 40.92 & 16.5 & 64.2 & 333 \\
\hline 63 & 0.2 & 40.9 & 6.3 & 78.9 & 348 \\
\hline 63 & 0.158 & 40.87 & 6.3 & 78.4 & 347 \\
\hline 63 & 0.158 & 41.03 & 15.3 & 65.5 & 335 \\
\hline 63 & 0.126 & 40.87 & 6.3 & 78.4 & 347 \\
\hline 63 & 0.126 & 41 & 15.3 & 65.5 & 335 \\
\hline 63 & 0.1 & 40.95 & 9.4 & 90.7 & 360 \\
\hline 63 & 0.079 & 40.96 & 9.4 & 90.7 & 360 \\
\hline 66 & 1.585 & 70.98 & 15 & 17.1 & 288 \\
\hline 66 & 1.259 & 71.53 & 15.4 & 17.6 & 288 \\
\hline 66 & 1 & 70.9 & 11.4 & 17.5 & 288 \\
\hline 66 & 0.794 & 71.46 & 13.9 & 19.2 & 290 \\
\hline 66 & 0.631 & 71.29 & 13.9 & 19.2 & 290 \\
\hline 69 & 1.259 & 7.32 & 7.7 & 172.8 & 84 \\
\hline 69 & 1 & 6.91 & 3.9 & 177.7 & 90 \\
\hline 69 & 0.794 & 7.12 & 3.9 & 177.7 & 90 \\
\hline 69 & 0.631 & 6.93 & 3 & 161.5 & 71 \\
\hline 69 & 0.501 & 7.33 & 10.2 & 169.2 & 80 \\
\hline 72 & 1.259 & 17.39 & 5.5 & 169 & 260 \\
\hline 72 & 1 & 17.91 & 8.2 & 147.8 & 237 \\
\hline 72 & 0.794 & 18.24 & 4 & 157.2 & 247 \\
\hline 72 & 0.631 & 18.29 & 4 & 157.2 & 247 \\
\hline 72 & 0.501 & 17.86 & 8.2 & 147.8 & 237 \\
\hline 72 & 0.398 & 17.8 & 8.3 & 144.6 & 233 \\
\hline 72 & 0.316 & 17.93 & 8.3 & 144.6 & 233 \\
\hline 72 & 0.251 & 17.81 & 8.3 & 144.6 & 233 \\
\hline 72 & 0.2 & 17.95 & 8.3 & 144.6 & 233 \\
\hline 79 & 1.259 & 47.61 & 15.3 & 12.9 & 110 \\
\hline 79 & 1 & 47.9 & 19.2 & 170.6 & 85 \\
\hline 79 & 0.794 & 47.63 & 13.2 & 169.2 & 84 \\
\hline 79 & 0.631 & 47.95 & 13.3 & 154.4 & 66 \\
\hline 79 & 0.501 & 47.93 & 13.3 & 154.4 & 66 \\
\hline 79 & 0.398 & 47.65 & 12.5 & 159.7 & 73 \\
\hline 79 & 0.316 & 47.66 & 12.5 & 159.7 & 73 \\
\hline 79 & 0.251 & 47.71 & 12.2 & 169.7 & 84 \\
\hline 79 & 0.2 & 47.68 & 4.2 & 151.8 & 62 \\
\hline 79 & 0.2 & 47.88 & 21.4 & 173 & 87 \\
\hline 79 & 0.158 & 47.68 & 7.7 & 145.2 & 54 \\
\hline 88 & 1 & 25.91 & 3.5 & 43.8 & 316 \\
\hline 88 & 0.794 & 26.18 & 1.9 & 53.8 & 325 \\
\hline 88 & 0.631 & 25.86 & 3.5 & 43.8 & 316 \\
\hline 88 & 0.501 & 25.88 & 3.7 & 42.2 & 314 \\
\hline 88 & 0.398 & 26.16 & 5.1 & 46.8 & 318 \\
\hline 88 & 0.316 & 25.84 & 3 & 22.5 & 295 \\
\hline 97 & 0.794 & 22.21 & 18 & 152.9 & 60 \\
\hline 97 & 0.631 & 22.08 & 20.6 & 154.5 & 62 \\
\hline 97 & 0.501 & 22.37 & 16.1 & 141 & 45 \\
\hline 97 & 0.398 & 22.18 & 16.8 & 151.1 & 58 \\
\hline 97 & 0.398 & 22.57 & 3.1 & 165.1 & 75 \\
\hline 101 & 0.794 & 36.51 & 4.5 & 165 & 258 \\
\hline 101 & 0.631 & 35.96 & 2.7 & 170.3 & 264 \\
\hline 101 & 0.501 & 36.4 & 5.5 & 162.8 & 255 \\
\hline 101 & 0.398 & 36.51 & 5.5 & 162.8 & 255 \\
\hline 109 & 0.631 & 35.33 & 11.8 & 71.7 & 162 \\
\hline 109 & 0.501 & 35.4 & 12.9 & 60.7 & 152 \\
\hline 109 & 0.398 & 35.31 & 13.2 & 56.1 & 148 \\
\hline 109 & 0.316 & 35.32 & 13.8 & 56.3 & 148 \\
\hline 109 & 0.251 & 35.4 & 15.3 & 57.9 & 150 \\
\hline 124 & 0.398 & 4.66 & 6.9 & 41.3 & 135 \\
\hline 124 & 0.316 & 4.65 & 8.1 & 58.3 & 152 \\
\hline 124 & 0.251 & 4.75 & 9.1 & 56.7 & 150 \\
\hline 130 & 0.398 & 24.56 & 6.1 & 179.3 & 271 \\
\hline 130 & 0.316 & 24.57 & 7.8 & 17.5 & 289 \\
\hline 130 & 0.251 & 24.6 & 12.4 & 17.3 & 289 \\
\hline 130 & 0.2 & 24.53 & 8.9 & 25.2 & 297 \\
\hline 143 & 0.316 & 22.99 & 15.4 & 1.5 & 93 \\
\hline 143 & 0.251 & 22.84 & 15.4 & 23.1 & 116 \\
\hline
\end{tabular}




\begin{tabular}{l|c|c|c|c|c}
204 & 0.158 & 47.84 & 21.4 & 173 & 87 \\
204 & 0.126 & 47.79 & 19.1 & 179.5 & 94 \\
204 & 0.1 & 47.85 & 19.4 & 169.2 & 83 \\
204 & 0.079 & 47.87 & 19.8 & 171.2 & 85 \\
204 & 0.063 & 47.88 & 19.8 & 171.2 & 85 \\
204 & 0.05 & 47.86 & 19 & 167.6 & 81 \\
211 & 0.126 & 47.67 & 12.8 & 120.1 & 23 \\
211 & 0.1 & 47.65 & 13 & 116.5 & 18 \\
211 & 0.079 & 47.63 & 13.2 & 135.2 & 42 \\
211 & 0.063 & 47.63 & 14.5 & 114.1 & 15 \\
212 & 0.126 & 47.92 & 6.6 & 46.5 & 316 \\
212 & 0.1 & 47.95 & 10.3 & 23.4 & 293 \\
212 & 0.079 & 47.95 & 10.3 & 23.4 & 293 \\
212 & 0.063 & 47.95 & 10.3 & 23.4 & 293 \\
212 & 0.05 & 47.96 & 13.1 & 29.5 & 298 \\
212 & 0.04 & 47.95 & 13.1 & 29.5 & 298 \\
217 & 0.1 & 24.56 & 3 & 26.3 & 298 \\
217 & 0.079 & 24.59 & 8.5 & 11.9 & 284 \\
217 & 0.063 & 24.6 & 9.4 & 9.1 & 281 \\
217 & 0.05 & 24.6 & 9.4 & 9.1 & 281 \\
217 & 0.04 & 24.58 & 9.4 & 9.1 & 281 \\
217 & 0.032 & 24.59 & 9.4 & 9.1 & 281 \\
223 & 0.1 & 47.75 & 6.5 & 29.3 & 124 \\
223 & 0.079 & 47.79 & 4 & 16.3 & 111 \\
223 & 0.063 & 47.76 & 6.8 & 25.6 & 121 \\
223 & 0.05 & 47.76 & 6.8 & 25.6 & 121 \\
223 & 0.04 & 47.75 & 6.5 & 16.5 & 112 \\
223 & 0.032 & 47.74 & 5.2 & 23.4 & 119 \\
223 & 0.032 & 47.77 & 2.9 & 28.7 & 122 \\
229 & 0.079 & 24.2 & 9.8 & 166.6 & 77 \\
229 & 0.063 & 24.22 & 9.8 & 166.6 & 77 \\
229 & 0.05 & 24.2 & 15.3 & 164.2 & 74 \\
229 & 0.04 & 24.23 & 9.6 & 170.8 & 81 \\
229 & 0.032 & 24.21 & 17.6 & 168.8 & 79 \\
229 & 0.025 & 24.21 & 15.1 & 167 & 77 \\
245 & 0.05 & 25.5 & 3.6 & 8.9 & 281 \\
245 & 0.05 & 25.55 & 10.6 & 1.7 & 274 \\
245 & 0.04 & 25.58 & 8.7 & 4.9 & 277 \\
245 & 0.032 & 25.57 & 10.6 & 1.7 & 274 \\
245 & 0.025 & 25.56 & 11.1 & 168.3 & 259 \\
272 & 0.025 & 47.72 & 3.9 & 109.7 & 194 \\
272 & 0.02 & 47.72 & 4.3 & 134 & 220 \\
272 & 0.016 & 47.71 & 4 & 147.8 & 236 \\
272 & 0.013 & 47.71 & 4 & 147.8 & 236 \\
272 & 0.01 & 47.71 & 4 & 147.8 & 236 \\
& & & & & \\
& & & &
\end{tabular}




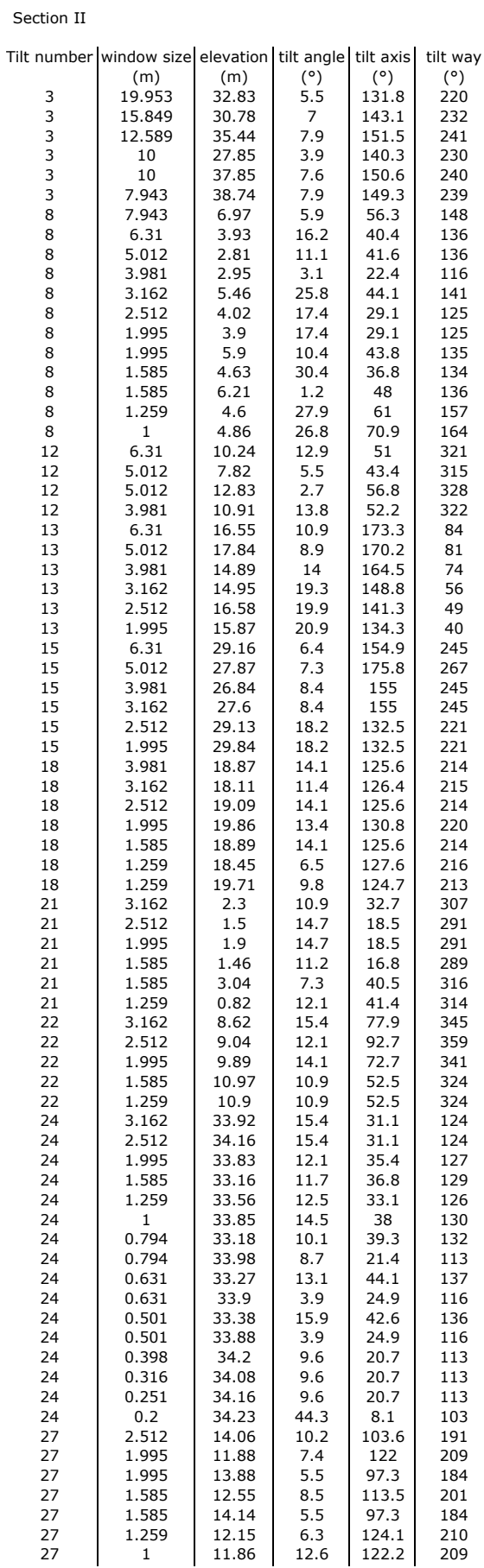




\begin{tabular}{|c|c|c|c|c|c|}
\hline 44 & 0.316 & 7.52 & 20.7 & 18.6 & 285 \\
\hline 44 & 0.316 & 7.83 & 15.9 & 36.1 & 306 \\
\hline 44 & 0.251 & 7.53 & 20.7 & 18.6 & 285 \\
\hline 44 & 0.2 & 7.49 & 20.7 & 18.6 & 285 \\
\hline 45 & 1 & 8.86 & 14 & 136.1 & 45 \\
\hline 45 & 0.794 & 8.56 & 6.4 & 145 & 55 \\
\hline 45 & 0.631 & 8.66 & 18.5 & 121.5 & 28 \\
\hline 45 & 0.501 & 8.82 & 23.2 & 109.1 & 15 \\
\hline 45 & 0.398 & 8.72 & 23.2 & 109.1 & 15 \\
\hline 66 & 0.631 & 32.63 & 6.9 & 51.5 & 322 \\
\hline 66 & 0.501 & 32.88 & 7.2 & 53.3 & 325 \\
\hline 66 & 0.398 & 32.61 & 7.4 & 67.8 & 337 \\
\hline 66 & 0.316 & 32.5 & 6 & 60 & 330 \\
\hline 66 & 0.316 & 32.82 & 6 & 50.3 & 323 \\
\hline 66 & 0.251 & 32.4 & 8.3 & 52.7 & 322 \\
\hline 66 & 0.2 & 32.43 & 8.2 & 35.6 & 307 \\
\hline 66 & 0.158 & 32.44 & 8.2 & 35.6 & 307 \\
\hline 66 & 0.126 & 32.42 & 9.2 & 39.8 & 311 \\
\hline 66 & 0.1 & 32.45 & 8.6 & 35.1 & 307 \\
\hline 66 & 0.079 & 32.47 & 8.6 & 35.1 & 307 \\
\hline 84 & 0.316 & 32.18 & 7.5 & 90.3 & 179 \\
\hline 84 & 0.251 & 32.15 & 10 & 79.5 & 169 \\
\hline 84 & 0.2 & 32.23 & 5.9 & 91.2 & 180 \\
\hline 84 & 0.158 & 32.13 & $\begin{array}{c}11.1 \\
\end{array}$ & 100.7 & 190 \\
\hline 84 & 0.126 & 32.17 & 10.8 & 89 & 178 \\
\hline 91 & 0.251 & 32.65 & 1.6 & 133.3 & 42 \\
\hline 91 & 0.2 & 32.63 & 3.7 & 138.9 & 46 \\
\hline 91 & 0.158 & 32.6 & 3.7 & 138.9 & 46 \\
\hline 91 & 0.126 & 32.55 & 1.7 & 123.8 & 30 \\
\hline 91 & 0.1 & 32.55 & 1.7 & 123.8 & 30 \\
\hline 92 & 0.251 & 32.9 & 9.4 & 36.9 & 309 \\
\hline 92 & 0.2 & 32.83 & 5.7 & 36.2 & 309 \\
\hline 92 & 0.158 & 32.76 & 2.7 & 46 & 319 \\
\hline 92 & 0.158 & 32.92 & 6.2 & 28.3 & 301 \\
\hline 92 & 0.126 & 32.8 & 6.8 & 54.5 & 325 \\
\hline 93 & 0.251 & 44.71 & 5.2 & 19.7 & 115 \\
\hline 93 & 0.2 & 44.6 & 7.9 & 44.2 & 139 \\
\hline 93 & 0.158 & 44.65 & 7.7 & 28.3 & 125 \\
\hline 93 & 0.126 & 44.63 & 9.1 & 43 & 139 \\
\hline 94 & 0.251 & 44.96 & 5.1 & 131.1 & 38 \\
\hline 94 & 0.2 & 45 & 8.1 & 136.6 & 44 \\
\hline 94 & 0.158 & 44.96 & 6.8 & 128.2 & 35 \\
\hline 94 & 0.126 & 45.01 & 12.1 & 148.3 & 57 \\
\hline 97 & 0.2 & 8.29 & 19.2 & 102.2 & 191 \\
\hline 97 & 0.158 & 8.19 & 20.6 & 89.3 & 177 \\
\hline 97 & 0.126 & 8.25 & 25.4 & 109.1 & 197 \\
\hline 97 & 0.1 & 8.26 & 25.4 & 109.1 & 197 \\
\hline 101 & 0.2 & 45.2 & 4.7 & 177.7 & 90 \\
\hline 101 & 0.158 & 45.28 & 17.1 & 1.7 & 95 \\
\hline 101 & 0.126 & 45.26 & 17.6 & 164.8 & 76 \\
\hline 102 & 0.2 & 45.4 & 22.2 & 129.9 & 219 \\
\hline 102 & 0.158 & 45.44 & 29.2 & 150.9 & 242 \\
\hline 102 & 0.126 & 45.52 & 20.3 & 173.8 & 268 \\
\hline 102 & 0.1 & 45.45 & 36.7 & 162.4 & 254 \\
\hline 102 & 0.079 & 45.41 & 28.7 & 144.1 & 234 \\
\hline 102 & 0.079 & 45.49 & 28 & 166.5 & 260 \\
\hline 102 & 0.063 & 45.41 & 28.7 & 144.1 & 234 \\
\hline 102 & 0.063 & 45.47 & 28 & 166.5 & 260 \\
\hline 102 & 0.05 & 45.38 & 24 & 131.6 & 222 \\
\hline 103 & 0.2 & 45.6 & 17.2 & 107.9 & 11 \\
\hline 103 & 0.158 & 45.6 & 19.8 & 127.2 & 30 \\
\hline 103 & 0.126 & 45.64 & 17.2 & 142.9 & 50 \\
\hline 103 & 0.1 & 45.55 & 12.8 & 154.8 & 67 \\
\hline 103 & 0.1 & 45.65 & 12.9 & 138.1 & 44 \\
\hline 103 & 0.079 & 45.57 & 12.8 & 154.8 & 67 \\
\hline 103 & 0.079 & 45.65 & 12.9 & 138.1 & 44 \\
\hline 110 & 0.158 & 45.12 & 7 & 158.1 & 248 \\
\hline 110 & 0.126 & 45.14 & 11.9 & 155.2 & 245 \\
\hline 110 & 0.1 & 45.15 & 8.7 & 175.5 & 267 \\
\hline 110 & 0.079 & 45.18 & 8.7 & 175.5 & 267 \\
\hline 110 & 0.063 & 45.16 & 10.5 & 170.8 & 262 \\
\hline 110 & 0.05 & 45.13 & 10.5 & 170.8 & 262 \\
\hline 119 & 0.126 & 44.76 & 3.5 & 106.4 & 12 \\
\hline 119 & 0.1 & 44.75 & 3.2 & 112.5 & 18 \\
\hline 119 & 0.079 & 44.78 & 14.8 & 110.5 & 15 \\
\hline 119 & 0.063 & 44.78 & 17.7 & 110.2 & 14 \\
\hline 119 & 0.05 & 44.78 & 17.7 & 110.2 & 14 \\
\hline 119 & 0.04 & 44.77 & 17.7 & 110.2 & 14 \\
\hline
\end{tabular}




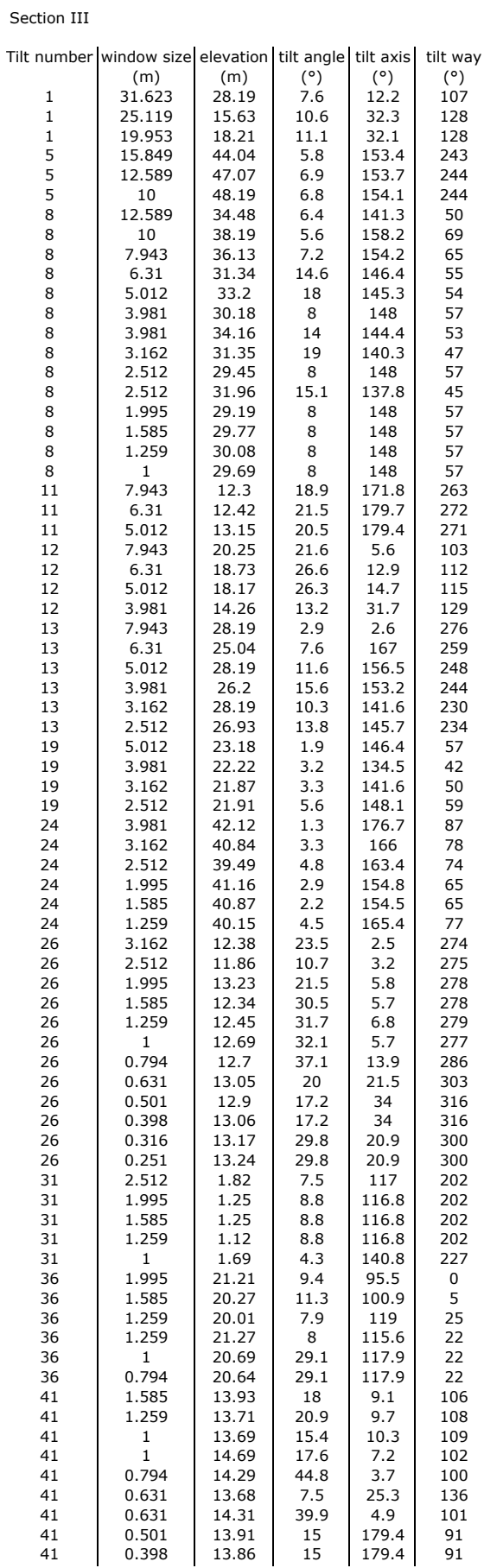




\begin{tabular}{|c|c|c|c|c|c|}
\hline 62 & 0.794 & 55.59 & 4.8 & 5.3 & 99 \\
\hline 62 & 0.631 & 55.32 & 6 & 179.1 & 93 \\
\hline 62 & 0.501 & 55.5 & 5.2 & 161.3 & 72 \\
\hline 62 & 0.398 & 55.66 & 8.2 & 138.9 & 47 \\
\hline 62 & 0.316 & 55.54 & 6.6 & 137 & 44 \\
\hline 62 & 0.251 & 55.44 & 5.6 & 140.2 & 48 \\
\hline 62 & 0.251 & 55.7 & 4.8 & 146.9 & 55 \\
\hline 62 & 0.2 & 55.43 & 4.2 & 145.2 & 53 \\
\hline 62 & 0.2 & 55.62 & 6.7 & 139 & 47 \\
\hline 62 & 0.158 & 55.61 & 4.1 & 132.2 & 40 \\
\hline 62 & 0.126 & 55.63 & 7.2 & 116.1 & 23 \\
\hline 63 & 0.794 & 1.58 & 5.3 & 169.1 & 262 \\
\hline 63 & 0.631 & 1.69 & 5.3 & 169.1 & 262 \\
\hline 63 & 0.501 & 1.38 & 15.7 & 161.3 & 253 \\
\hline 63 & 0.398 & 1.12 & 7.5 & 136.5 & 223 \\
\hline 63 & 0.398 & 1.52 & 9 & 158.7 & 250 \\
\hline 63 & 0.316 & 1.15 & 11.2 & 162.6 & 254 \\
\hline 63 & 0.251 & 1.19 & 11.2 & 162.6 & 254 \\
\hline 67 & 0.794 & 23.03 & 23.8 & 152.2 & 242 \\
\hline 67 & 0.631 & 22.51 & 32.6 & 159.4 & 250 \\
\hline 67 & 0.501 & 22.93 & 29.7 & 153.8 & 244 \\
\hline 71 & 0.631 & 11.79 & 9.1 & 167.6 & 77 \\
\hline 71 & 0.501 & 11.9 & 17.2 & 8.6 & 102 \\
\hline 71 & 0.398 & 11.87 & 16.4 & 4.9 & 97 \\
\hline 71 & 0.316 & 11.9 & 20.7 & 16.5 & 112 \\
\hline 71 & 0.251 & 11.99 & 23.9 & 2.9 & 94 \\
\hline 71 & 0.2 & 11.93 & 25.6 & 17.3 & 112 \\
\hline 71 & 0.158 & 11.87 & 10.6 & 38.4 & 136 \\
\hline 71 & 0.126 & 11.95 & 26.1 & 15.6 & 111 \\
\hline 71 & 0.1 & 11.99 & 23.3 & 171.5 & 82 \\
\hline 72 & 0.631 & 12.42 & 24.3 & 168.5 & 258 \\
\hline 72 & 0.501 & 12.4 & 29.1 & 173.2 & 264 \\
\hline 72 & 0.398 & 12.27 & 28.7 & 3 & 274 \\
\hline 72 & 0.316 & 12.54 & 30.1 & 166.6 & 256 \\
\hline 72 & 0.251 & 12.24 & 38.9 & 1 & 271 \\
\hline 72 & 0.2 & 12.33 & 40.2 & 1.5 & 272 \\
\hline 72 & 0.158 & 12.34 & 56 & 176.6 & 267 \\
\hline 72 & 0.126 & 12.33 & 56 & 176.6 & 267 \\
\hline 80 & 0.631 & 41.44 & 5.3 & 171.1 & 262 \\
\hline 80 & 0.501 & 41.47 & 7.2 & 164.5 & 255 \\
\hline 80 & 0.398 & 41.73 & 3.4 & 179 & 270 \\
\hline 80 & 0.316 & 41.63 & 7.2 & 164.5 & 255 \\
\hline 84 & 0.501 & 0.37 & 5.5 & 54.8 & 149 \\
\hline 84 & 0.398 & 0.32 & 6.5 & 44.7 & 140 \\
\hline 84 & 0.316 & 0.2 & 9.8 & 31.8 & 129 \\
\hline 84 & 0.251 & 0.18 & 12.6 & 26.8 & 123 \\
\hline 84 & 0.2 & 0.16 & 11.4 & 21.8 & 117 \\
\hline 84 & 0.158 & 0.14 & 11.4 & 21.8 & 117 \\
\hline 84 & 0.126 & 0.24 & 8.9 & 11.4 & 106 \\
\hline 84 & 0.1 & 0.19 & 8.9 & 11.4 & 106 \\
\hline 88 & 0.501 & 11.4 & 8.8 & 15.3 & 288 \\
\hline 88 & 0.398 & 11.47 & 7.3 & 14.1 & 287 \\
\hline 88 & 0.316 & 11.59 & 10.1 & 17.3 & 291 \\
\hline 90 & 0.501 & 14.41 & 39.9 & 4.9 & 101 \\
\hline 90 & 0.398 & 14.26 & 48.4 & 4.4 & 100 \\
\hline 90 & 0.316 & 14.43 & 49.6 & 5.8 & 102 \\
\hline 90 & 0.251 & 14.5 & 49.6 & 5.8 & 102 \\
\hline 94 & 0.501 & 39.47 & 9.1 & 176.4 & 90 \\
\hline 94 & 0.398 & 39.34 & 10.2 & 14.5 & 109 \\
\hline 94 & 0.316 & 39.42 & 10.2 & 14.5 & 109 \\
\hline 94 & 0.251 & 39.37 & 5.6 & 25.8 & 121 \\
\hline 94 & 0.251 & 39.62 & 9.6 & 1.3 & 95 \\
\hline 95 & 0.501 & 39.97 & 4.6 & 70.2 & 339 \\
\hline 95 & 0.398 & 39.74 & 10.4 & 77.2 & 345 \\
\hline 95 & 0.316 & 39.73 & 10.3 & 83.2 & 350 \\
\hline 97 & 0.501 & 42.47 & 2 & 142.9 & 232 \\
\hline 97 & 0.398 & 42.12 & 2.3 & 126.6 & 215 \\
\hline 97 & 0.316 & 42.26 & 6.1 & 143.3 & 233 \\
\hline 97 & 0.251 & 42.13 & 4.2 & 136.2 & 225 \\
\hline 97 & 0.251 & 42.38 & 4 & 157.8 & 248 \\
\hline 97 & 0.2 & 42.26 & 6.1 & 143.3 & 233 \\
\hline 97 & 0.158 & 42.14 & 4.2 & 136.2 & 225 \\
\hline 97 & 0.158 & 42.3 & 4 & 157.8 & 248 \\
\hline 97 & 0.126 & 42.16 & 4.2 & 136.2 & 225 \\
\hline 97 & 0.1 & 42.19 & 4.2 & 136.2 & 225 \\
\hline 122 & 0.316 & 56.18 & 18.3 & 162.2 & 254 \\
\hline 122 & 0.251 & 56.2 & 10.1 & 178.1 & 272 \\
\hline 122 & 0.2 & 56.22 & 12.5 & 8 & 281 \\
\hline 134 & 0.251 & 55.19 & 9.4 & 94.4 & 182 \\
\hline
\end{tabular}




\begin{tabular}{l|c|c|c|c|c}
160 & 0.063 & 56.08 & 28.7 & 144.1 & 234 \\
160 & 0.063 & 56.14 & 28 & 166.5 & 260 \\
171 & 0.126 & 56.01 & 18.9 & 21.7 & 116 \\
171 & 0.1 & 55.99 & 25.6 & 10.5 & 104 \\
171 & 0.079 & 55.99 & 34.3 & 176.1 & 88 \\
171 & 0.063 & 56.02 & 34.3 & 176.1 & 88 \\
171 & 0.05 & 55.98 & 34.3 & 176.1 & 88 \\
171 & 0.04 & 56 & 34.3 & 176.1 & 88 \\
172 & 0.126 & 56.26 & 25.6 & 134.4 & 37 \\
172 & 0.1 & 56.29 & 27.1 & 124.6 & 26 \\
172 & 0.079 & 56.31 & 27.1 & 124.6 & 26 \\
172 & 0.063 & 56.27 & 27.8 & 121.1 & 21 \\
172 & 0.05 & 56.28 & 27.8 & 121.1 & 21 \\
172 & 0.04 & 56.28 & 27.8 & 121.1 & 21 \\
183 & 0.1 & 55.69 & 12.1 & 148.3 & 57 \\
183 & 0.079 & 55.67 & 16.7 & 139.4 & 47 \\
183 & 0.063 & 55.7 & 19.5 & 143 & 50 \\
183 & 0.05 & 55.68 & 19.5 & 143 & 50 \\
184 & 0.1 & 55.79 & 13.9 & 154.7 & 244 \\
184 & 0.079 & 55.83 & 8.7 & 175.5 & 267 \\
184 & 0.063 & 55.83 & 10.5 & 170.8 & 262
\end{tabular}


Section I

Tilt number / window size elevation / tilt angle tilt axis tilt way

\begin{tabular}{|c|c|c|c|c|c|}
\hline & (m) & (m) & (०) & $\left({ }^{\circ}\right)$ & $\left(^{\circ}\right)$ \\
\hline 1 & 31.623 & 21.09 & 7.2 & 29.4 & 123 \\
\hline 2 & 10 & 56.9 & 10.3 & 135.4 & 44 \\
\hline 2 & 10 & 66.9 & 7.7 & 141.9 & 51 \\
\hline 3 & 3.162 & 14.77 & 13.3 & 161.1 & 70 \\
\hline 6 & 5.012 & 44.42 & 14.7 & 141.6 & 231 \\
\hline 7 & 7.943 & 28.96 & 5.6 & 91.1 & 179 \\
\hline 9 & 3.981 & 11.03 & 4.6 & 159.3 & 249 \\
\hline 10 & 3.981 & 38.9 & 11.5 & 145.4 & 56 \\
\hline 11 & 3.981 & 66.76 & 7.6 & 156.2 & 66 \\
\hline 13 & 2.512 & 24.35 & 4.3 & 129.5 & 218 \\
\hline 13 & 2.512 & 26.86 & 5.5 & 133.5 & 221 \\
\hline 15 & 1.995 & 1.99 & 9.3 & 42.7 & 315 \\
\hline 21 & 3.981 & 42.88 & 16.4 & 9.2 & 280 \\
\hline 22 & 1.585 & 47.21 & 23.7 & 79 & 172 \\
\hline 27 & 1.995 & 11.96 & 12.7 & 175.1 & 267 \\
\hline 34 & 1.585 & 6 & 5.3 & 25.2 & 120 \\
\hline 43 & 1.585 & 7.58 & 17.7 & 15.9 & 289 \\
\hline 48 & 0.631 & 24.6 & 10.6 & 4.2 & 276 \\
\hline 49 & 0.794 & 34.92 & 6.2 & 111.2 & 200 \\
\hline 49 & 0.794 & 35.71 & 5.3 & 88.3 & 175 \\
\hline 53 & 1 & 48.9 & 15.8 & 174.8 & 266 \\
\hline 53 & 1 & 49.9 & 2.6 & 153.7 & 246 \\
\hline 63 & 0.158 & 40.87 & 6.3 & 78.4 & 347 \\
\hline 63 & 0.158 & 41.03 & 15.3 & 65.5 & 335 \\
\hline 66 & 1.259 & 71.53 & 15.4 & 17.6 & 288 \\
\hline 69 & 0.501 & 7.33 & 10.2 & 169.2 & 80 \\
\hline 72 & 0.2 & 17.95 & 8.3 & 144.6 & 233 \\
\hline 79 & 0.2 & 47.68 & 4.2 & 151.8 & 62 \\
\hline 79 & 0.2 & 47.88 & 21.4 & 173 & 87 \\
\hline 88 & 0.398 & 26.16 & 5.1 & 46.8 & 318 \\
\hline 97 & 0.631 & 22.08 & 20.6 & 154.5 & 62 \\
\hline 101 & 0.398 & 36.51 & 5.5 & 162.8 & 255 \\
\hline 109 & 0.251 & 35.4 & 15.3 & 57.9 & 150 \\
\hline 124 & 0.251 & 4.75 & 9.1 & 56.7 & 150 \\
\hline 130 & 0.251 & 24.6 & 12.4 & 17.3 & 289 \\
\hline 143 & 0.126 & 22.87 & 25.5 & 20.3 & 115 \\
\hline 144 & 0.2 & 24.33 & 7.1 & 66 & 156 \\
\hline 152 & 0.2 & 6.78 & 9.4 & 6.8 & 100 \\
\hline 157 & 0.2 & 25.73 & 10.2 & 9.3 & 103 \\
\hline 193 & 0.158 & 24.23 & 11.4 & 176.1 & 87 \\
\hline 194 & 0.079 & 24.35 & 14.3 & 142.6 & 233 \\
\hline 195 & 0.12 & 24.5 & 9.9 & 67.7 & 336 \\
\hline 204 & 0.158 & 47.84 & 21.4 & 173 & 87 \\
\hline 211 & 0.063 & 47.63 & 14.5 & 114.1 & 15 \\
\hline 212 & 0.04 & 47.95 & 13.1 & 29.5 & 298 \\
\hline 217 & 0.032 & 24.59 & 9.4 & 9.1 & 281 \\
\hline 223 & 0.032 & 47.74 & 5.2 & 23.4 & 119 \\
\hline 223 & 0.032 & 47.77 & 2.9 & 28.7 & 122 \\
\hline 229 & 0.032 & 24.21 & 17.6 & 168.8 & 79 \\
\hline 245 & 0.05 & 25.5 & 3.6 & 8.9 & 281 \\
\hline 245 & 0.05 & 25.55 & 10.6 & 1.7 & 274 \\
\hline 272 & 0.02 & 47.72 & 4.3 & 134 & 220 \\
\hline
\end{tabular}

Section II

\begin{tabular}{|c|c|c|c|c|c|}
\hline It number & $\mid \begin{array}{c}\text { window size } \\
(\mathrm{m})\end{array}$ & $\begin{array}{c}\text { elevation } \\
(\mathrm{m})\end{array}$ & $\mid \begin{array}{c}\text { tilt angle } \\
\left({ }^{\circ}\right)\end{array}$ & $\left|\begin{array}{c}\text { tilt axis } \\
\left({ }^{\circ}\right)\end{array}\right|$ & $\begin{array}{c}\text { tilt way } \\
\left({ }^{\circ}\right)\end{array}$ \\
\hline 3 & 10 & 27.85 & 3.9 & 140.3 & 230 \\
\hline 3 & 10 & 37.85 & 7.6 & 150.6 & 240 \\
\hline 8 & 1.585 & 4.63 & 30.4 & 36.8 & 134 \\
\hline 8 & 1.585 & 6.21 & 1.2 & 48 & 136 \\
\hline 12 & 3.981 & 10.91 & 13.8 & 52.2 & 322 \\
\hline 13 & 1.995 & 15.87 & 20.9 & 134.3 & 40 \\
\hline 15 & 1.995 & 29.84 & 18.2 & 132.5 & 221 \\
\hline 18 & 1.259 & 18.45 & 6.5 & 127.6 & 216 \\
\hline 18 & 1.259 & 19.71 & 9.8 & 124.7 & 213 \\
\hline 21 & 1.585 & 1.46 & 11.2 & 16.8 & 289 \\
\hline 21 & 1.585 & 3.04 & 7.3 & 40.5 & 316 \\
\hline 22 & 3.162 & 8.62 & 15.4 & 77.9 & 345 \\
\hline 24 & 0.2 & 34.23 & 44.3 & 8.1 & 103 \\
\hline 27 & 1.585 & 12.55 & 8.5 & 113.5 & 201 \\
\hline 27 & 1.585 & 14.14 & 5.5 & 97.3 & 184 \\
\hline
\end{tabular}

Section III

Tilt number $/$ window size elevation t tilt angle tilt axis tilt way

\begin{tabular}{|c|c|c|c|c|c|}
\hline & (m) & (m) & $\left({ }^{\circ}\right)$ & $\left({ }^{\circ}\right)$ & $\left({ }^{\circ}\right)$ \\
\hline 1 & 19.953 & 18.21 & 11.1 & 32.1 & 128 \\
\hline 5 & 12.589 & 47.07 & 6.9 & 153.7 & 244 \\
\hline 8 & 2.512 & 29.45 & 8 & 148 & 57 \\
\hline 8 & 2.512 & 31.96 & 15.1 & 137.8 & 45 \\
\hline 11 & 6.31 & 12.42 & 21.5 & 179.7 & 272 \\
\hline 12 & 6.31 & 18.73 & 26.6 & 12.9 & 112 \\
\hline 13 & 3.981 & 26.2 & 15.6 & 153.2 & 244 \\
\hline 19 & 2.512 & 21.91 & 5.6 & 148.1 & 59 \\
\hline 24 & 2.512 & 39.49 & 4.8 & 163.4 & 74 \\
\hline 26 & 0.794 & 12.7 & 37.1 & 13.9 & 286 \\
\hline 31 & 1.259 & 1.12 & 8.8 & 116.8 & 202 \\
\hline 36 & 0.794 & 20.64 & 29.1 & 117.9 & 22 \\
\hline 41 & 0.631 & 13.68 & 7.5 & 25.3 & 136 \\
\hline 41 & 0.631 & 14.31 & 39.9 & 4.9 & 101 \\
\hline 44 & 0.398 & 23.41 & 17.9 & 158.5 & 71 \\
\hline 49 & 0.794 & 21.44 & 22.9 & 150.5 & 240 \\
\hline 52 & 0.398 & 43.72 & 4.4 & 49.8 & 141 \\
\hline 53 & 0.794 & 0.79 & 8.8 & 67.7 & 160 \\
\hline 62 & 0.2 & 55.43 & 4.2 & 145.2 & 53 \\
\hline 62 & 0.2 & 55.62 & 6.7 & 139 & 47 \\
\hline 63 & 0.398 & 1.12 & 7.5 & 136.5 & 223 \\
\hline 63 & 0.398 & 1.52 & 9 & 158.7 & 250 \\
\hline 67 & 0.631 & 22.51 & 32.6 & 159.4 & 250 \\
\hline 71 & 0.2 & 11.93 & 25.6 & 17.3 & 112 \\
\hline 72 & 0.126 & 12.33 & 56 & 176.6 & 267 \\
\hline 80 & 0.316 & 41.63 & 7.2 & 164.5 & 255 \\
\hline 84 & 0.251 & 0.18 & 12.6 & 26.8 & 123 \\
\hline 88 & 0.316 & 11.59 & 10.1 & 17.3 & 291 \\
\hline 90 & 0.251 & 14.5 & 49.6 & 5.8 & 102 \\
\hline 94 & 0.316 & 39.42 & 10.2 & 14.5 & 109 \\
\hline 95 & 0.398 & 39.74 & 10.4 & 77.2 & 345 \\
\hline 97 & 0.158 & 42.14 & 4.2 & 136.2 & 225 \\
\hline 97 & 0.158 & 42.3 & 4 & 157.8 & 248 \\
\hline 122 & 0.316 & 56.18 & 18.3 & 162.2 & 254 \\
\hline 134 & 0.079 & 55.28 & 10.9 & 48.6 & 143 \\
\hline 137 & 0.1 & 0.59 & 8.3 & 13.8 & 109 \\
\hline 141 & 0.158 & 12.02 & 16.9 & 178.7 & 91 \\
\hline 141 & 0.158 & 12.18 & 26.4 & 163.6 & 74 \\
\hline 146 & 0.158 & 42.93 & 2 & 163.7 & 75 \\
\hline 146 & 0.158 & 43.09 & 3.5 & 179.2 & 91 \\
\hline 160 & 0.063 & 56.08 & 28.7 & 144.1 & 234 \\
\hline 160 & 0.063 & 56.14 & 28 & 166.5 & 260 \\
\hline 171 & 0.04 & 56 & 34.3 & 176.1 & 88 \\
\hline 172 & 0.04 & 56.28 & 27.8 & 121.1 & 21 \\
\hline 183 & 0.05 & 55.68 & 19.5 & 143 & 50 \\
\hline 184 & 0.1 & 55.79 & 13.9 & 154.7 & 244 \\
\hline
\end{tabular}

